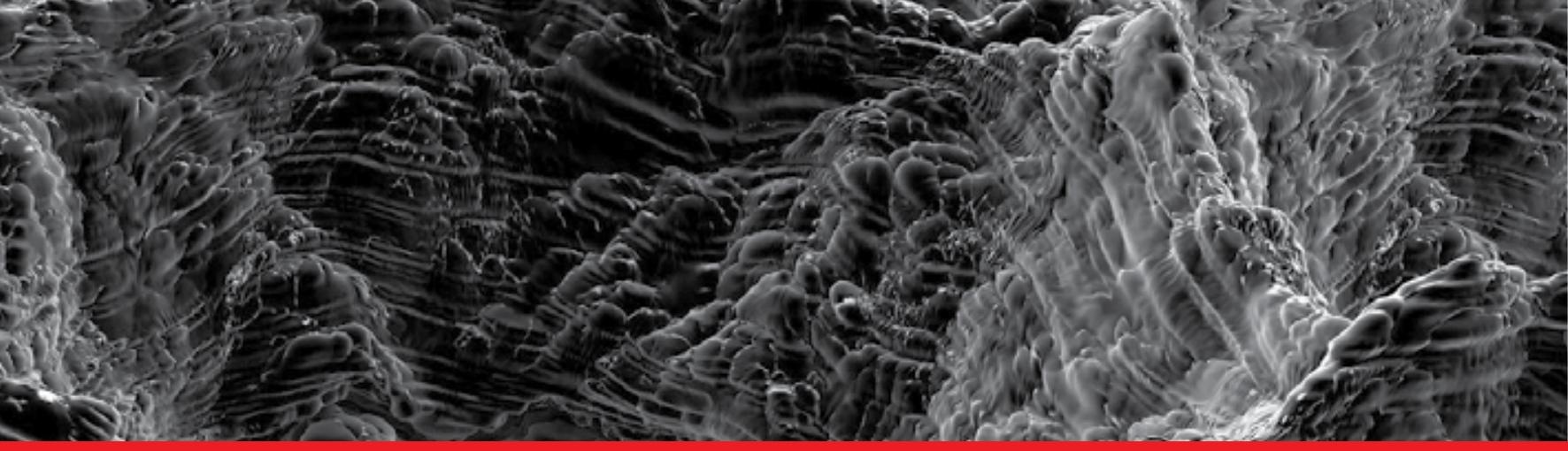

\title{
IntechOpen
}

\section{Micro and Nanotechnologies for Biotechnology}

Edited by Stefan G. Stanciu

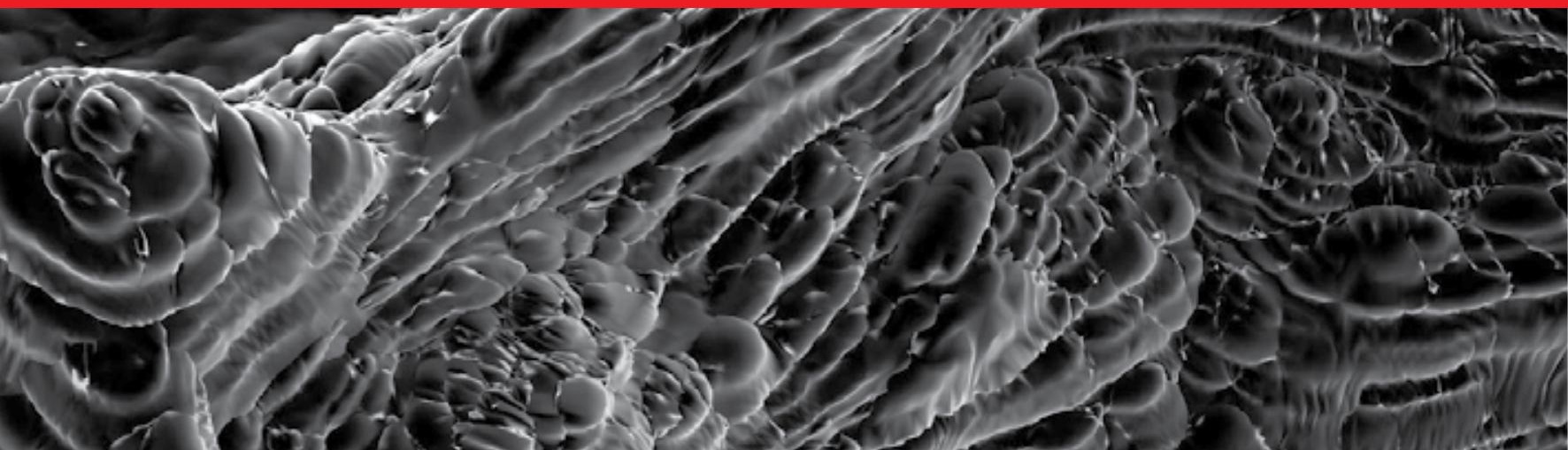





\section{MICRO AND \\ NANOTECHNOLOGIES \\ FOR BIOTECHNOLOGY}

Edited by Stefan G. Stanciu 
Micro and Nanotechnologies for Biotechnology

http://dx.doi.org/10.5772/61530

Edited by Stefan G. Stanciu

\section{Contributors}

Ming-Kung Yeh, Yuan-Chuan Chen, Hwei-Fang Cheng, Yi-Chen Yang, Lavinia Cosmina Ardelean, Lucien Reclaru, Cristina Maria Bortun, Laura Cristina Rusu, Hong Wu, Tiehong Yang, Li Fan, Camilla Thorling, Amy Holmes, Hauke Studier, Xiaowen Liang, David Liu, Michael Roberts, Maja Leitgeb, Željko Knez, Katja Vasić, Loredana Latterini, Luigi Tarpani

\section{(c) The Editor(s) and the Author(s) 2016}

The moral rights of the and the author(s) have been asserted.

All rights to the book as a whole are reserved by INTECH. The book as a whole (compilation) cannot be reproduced, distributed or used for commercial or non-commercial purposes without INTECH's written permission.

Enquiries concerning the use of the book should be directed to INTECH rights and permissions department (permissions@intechopen.com).

Violations are liable to prosecution under the governing Copyright Law.

\section{(cc) BY}

Individual chapters of this publication are distributed under the terms of the Creative Commons Attribution 3.0 Unported License which permits commercial use, distribution and reproduction of the individual chapters, provided the original author(s) and source publication are appropriately acknowledged. If so indicated, certain images may not be included under the Creative Commons license. In such cases users will need to obtain permission from the license holder to reproduce the material. More details and guidelines concerning content reuse and adaptation can be foundat http://www.intechopen.com/copyright-policy.html.

\section{Notice}

Statements and opinions expressed in the chapters are these of the individual contributors and not necessarily those of the editors or publisher. No responsibility is accepted for the accuracy of information contained in the published chapters. The publisher assumes no responsibility for any damage or injury to persons or property arising out of the use of any materials, instructions, methods or ideas contained in the book.

First published in Croatia, 2016 by INTECH d.o.o.

eBook (PDF) Published by IN TECH d.o.o.

Place and year of publication of eBook (PDF): Rijeka, 2019.

IntechOpen is the global imprint of IN TECH d.o.o.

Printed in Croatia

Legal deposit, Croatia: National and University Library in Zagreb

Additional hard and PDF copies can be obtained from orders@intechopen.com

Micro and Nanotechnologies for Biotechnology

Edited by Stefan G. Stanciu

p. cm.

Print ISBN 978-953-51-2530-3

Online ISBN 978-953-51-2531-0

eBook (PDF) ISBN 978-953-51-5785-4 


\section{We are IntechOpen, the first native scientific \\ publisher of Open Access books}

$3,350+$

Open access books available

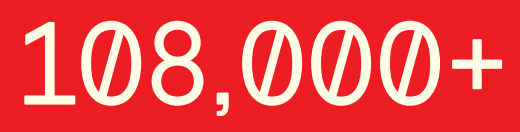

International authors and editors

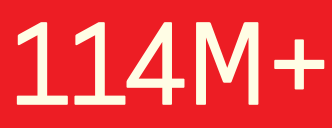

Downloads

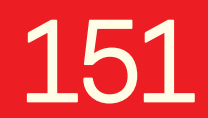

Countries delivered to

Our authors are among the

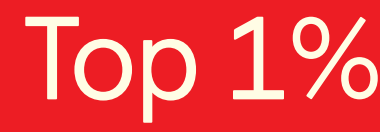

most cited scientists

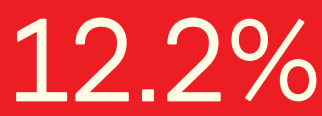

Contributors from top 500 universities

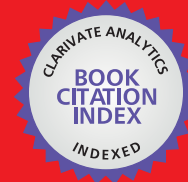

WEB OF SCIENCE ${ }^{\text {TM }}$

Selection of our books indexed in the Book Citation Index in Web of Science ${ }^{\mathrm{TM}}$ Core Collection (BKCI)

Interested in publishing with us?

Contact book.department@intechopen.com

Numbers displayed above are based on latest data collected.

For more information visit www.intechopen.com 



\section{Meet the editor}

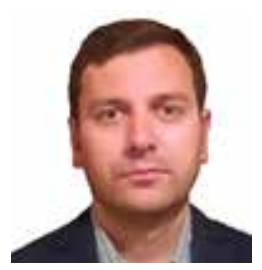

Dr. Stefan G. Stanciu received a PhD degree in Electronics and Telecommunications from the University Politehnica of Bucharest (UPB) in 2011. Currently, he is conducting his research activities as Scientific Researcher at the Center for Microscopy-Microanalysis and Information Processing of UPB. His main research interests revolve around scanning laser and scanning probe microscopies and connected image processing and computer vision topics. Stefan's current research agenda focuses not only on optical characterization of cells, tissues and biomaterials using combined biophotonics approaches but also on the design and development of novel image classification frameworks for automated disease diagnostics. 



\section{Contents}

Preface XI

Chapter 1 Interactions Between Plasmonic Nanostructures and Proteins 1

Loredana Latterini and Luigi Tarpani

Chapter 2 Micro- and Nanocarriers for Immobilization of Enzymes 21 Maja Leitgeb, Željko Knez and Katja Vasić

Chapter 3 Construction and Biological Evaluation of Nanoparticle-Based Tumor Targeting Drug Delivery Systems 59

Hong Wu, Tiehong Yang and Li Fan

Chapter 4 Nanotechnologies Applied in Biomedical Vaccines 85

Yuan-Chuan Chen, Hwei-Fang Cheng, Yi-Chen Yang and Ming-

Kung Yeh

Chapter 5 Multiphoton and Fluorescence Lifetime Imaging Microscopy in Studying Nanoparticle Pharmacokinetics in Skin and Liver 107 Camilla A. Thorling, Amy Holmes, Hauke Studier, David Liu, Xiaowen Liang and Michael S. Roberts

Chapter 6 Investigations on Dental Alloys Using Metallographic Observation, Scanning Electron Microscopy, and EnergyDispersive X-Ray Spectroscopy 125 Lavinia Ardelean, Lucien Reclaru, Cristina-Maria Bortun and LauraCristina Rusu 



\section{Preface}

The word "technology" has its origins in the Greek language, where the term "techne" refers to "skill", "craft" or "art", whereas "logos" means "word" or "to speak of". Thus, if we are to consider it literally, "technology" means expressing in words the way things are created, built, developed or gained. Although Merriam-Webster provides two simple and tidy definitions of "technology": (1) "the use of science in industry, engineering, etc., to invent useful things or to solve problems" and (2) "a machine, piece of equipment, method, etc., that is created by technology", pinning down a definition of the word that would please everyone is not as easy as it seems. Technology may refer to a technological process, a technological object, a technological system, technological knowledge or to a set of technological items. This complicates things in terms of providing a brief and objective answer to the question "What is technolo$g y$ ?" What is, however, clear and certain is that technologies are inseparable to human nature and that the modern society in which we live in today would not be where it is now without them.

In the title of this book, three terms based on the word "technology" can be found: "microtechnologies", "nanotechnologies" and "biotechnology". While at first this repetition may seem to be not too harmonious, if we give it more thought, we come to observe that these three words nicely blend and fit in next to each other. This is because over the past decades cutting-edge micro- and nanostructured materials, systems or devices have been successfully transferred to innovative applications oriented towards the "Bio" realm, which practically consists in everything that has to do with life. Such developments would not have been possible without the advent of a wide range of investigation techniques, methods and protocols that allow today the in-depth characterization of micro- and nanoscaled items and processes at sufficient resolutions to precisely understand their properties and characteristics; these tools are now considered to be of the utmost importance for biomedical research and practice. Moreover, countless healthcare and biomedical solutions with high impact in terms of timely diagnostics, therapeutic success, patient comfort or financial sustainability of healthcare systems around the world rely on micro- and nanotechnologies. Thus, it is not at all exaggerating to claim that such technologies play in current days a tremendous role with respect to improving the quality of our life, health and well-being, which are the main priorities of modern science. Taking a look at what has been happening in the biotechnology field in recent years, we can well figure that the number of applications and solutions based on micro- and nanotechnologies will exponentially rise in the forthcoming years, resulting in next-generation applications that will take critical areas such as medicine or biology by storm. The six chapters of this book represent, in my view, illustrative examples for these statements, addressing highly significant scientific subjects from diverse areas of micro- and nanotechnologies for biotechnology. Authoritative voices in their fields present in this vol- 
ume their work, or review recent trends, concepts and applications, in a manner that is accessible to quite a broad readership audience from both within and outside their specialist area. I am confident that this volume will be of great value not only to those actively involved in the addressed fields but also to those with passive but constant interest in these scientific areas and to those who will have their first encounter with micro- and nanotechnologies for biotechnology when reading the contained chapters. In the end, I like to express my deepest thanks to each of the authors for their fine contributions to this project.

Stefan G. Stanciu, PhD

Center for Microscopy-Microanalysis and Information Processing University Politehnica of Bucharest, Bucharest, Romania 


\title{
Chapter 1
}

\section{Interactions Between Plasmonic Nanostructures and Proteins}

\author{
Loredana Latterini and Luigi Tarpani \\ Additional information is available at the end of the chapter \\ http://dx.doi.org/10.5772/63454
}

\begin{abstract}
In the development of a nanodevice for biomedical applications, the study of the interactions with the biomolecules is essential. Proteins, in particular, are known to be easily adsorbed on the surface of the nanoparticles and the resulting complex is the one that will be effectively internalized by the target cells. Owing to the versatility of the preparation methods available and the unique optical properties, gold nanomaterials represent an excellent choice to study this interaction. This chapter will initially describe the synthesis of gold nanorods and nanoshells that are able to absorb light in the near-infrared (NIR) region. Then, the methods available for the functionalization of their surface will be discussed. The surface plasmon absorption will be used as an optical tool to monitor the process of preparation and surface modification. In the last section of the chapter, fluorescence and microscopy techniques will be used to follow the formation and characterize the protein-nanoparticle complex. The modifications of the emission spectra of two model proteins, bovine serum albumin (BSA) and myoglobin (Mb), will be analyzed in detail. The data will demonstrate that structural rearrangements following the adsorption on the surface of the nanoparticles are responsible for the changes in the fluorescence of the tryptophan residues of the protein. The data will be discussed in terms of static and dynamic quenching, proving the formation of a protein-nanoparticle complex. Atomic force microscopy (AFM) measurements will allow the direct visualization of this complex.
\end{abstract}

Keywords: gold nanostructures, protein adsorption, localized surface plasmon resonance, fluorescence, atomic force microscopy 


\section{Introduction}

In the last few decades, the use of nanotechnology in the biomedical field has grown exponentially. This interest is originated from the fact that materials at the nanometer scale have completely different properties than their bulk counterparts and these properties are dependent on the particle size [1,2]. Also, nanoparticles are ideal systems for application in bioimaging and therapy because they are small enough to interact with the biomolecules and to be efficiently internalized by the cells.

The first and most important process that occurs when a nanoparticle comes in contact with a biological fluid is the association with proteins. In the design of engineered nanostructures, this process has to be taken into account, because it may determine the fate of these species inside the living cells, their clearance rate and cytotoxicity. In fact, the systems that will be eventually delivered to the final biological target are protein-coated nanoparticles and their surface properties will affect their ability to cross the cell membrane barrier. Also, the adsorption of proteins on the surface of the particles may lead to conformational changes, unfolding and eventually to their irreversible denaturation [3-5]. These processes could alter the normal protein functions and cause unwanted side-effects for the organism. For all these reasons, it is clear that a full comprehension of the nature and the mechanism of this process of association can be of support in the development of optimized nanostructures for biomedical applications.

Gold-based nanomaterials (GNMs) are among the most studied systems to be employed as diagnostic and therapeutic tools for biomedical applications. This success comes from their unique properties. They are highly biocompatible and the preparation method can be easily adjusted to control their morphology and optical properties. They also have easy processable surfaces, which allow the conjugation with different ligands for the specific targeting to the cells of interest. Most importantly, when gold is reduced to the nanometric size, the interaction with light under certain experimental conditions produces a strong absorption in the visible region caused by the collective oscillation of the electron cloud on the surface of the metal. This effect, known as localized surface plasmon resonance (LSPR), is extensively used in biological sensing and imaging [6-10]. In fact, the frequency at which LSPR occurs and its intensity are dependent on size and shape of the nanoparticles, interparticle distance and the dielectric function of the surrounding Medium. All these physical parameters can change upon interaction of the GNMs with the biological species. The adsorption of protein on GNMs drastically modifies the surface properties of gold colloids and may eventually lead to their aggregation. These effects result in changes in the LSPR optical response that can be measured by ultravioletvisible (UV-Vis) spectroscopy.

Many other techniques are currently employed to study the interaction between proteins and nanoparticles, such as Fourier transform infrared spectroscopy (FTIR) [11, 12], nuclear magnetic resonance (NMR) [13], circular dichroism spectroscopy [12, 14], size-exclusion chromatography [15]. Among them, fluorescence is a powerful tool for probing the adsorption of proteins on the surface of nanoparticles because of its high sensibility in detecting even small changes in the microenvironment surrounding the emitting species. Additionally, the use of 
the intrinsic fluorescence of the proteins to monitor the association process makes unnecessary the attachment of an imaging probe that may perturb the system under investigation.

In this chapter, the interaction of GNMs with different model proteins in vitro is analyzed by UV-Vis spectroscopy and microscopy techniques. The first section describes the most common methods of preparation of GNMs with the focus on those systems whose morphology promotes a shift of the LSPR in the NIR region. An efficient absorption of the system in this portion of the electromagnetic spectrum is desirable because the light at these energies has the maximum penetration in the biological tissues. This section also illustrates the synthetic steps able to functionalize the GNMs surface in order to tune the interaction with the proteins. In the second section, the detailed study of the adsorption of the model proteins on the surface of the GNMs is reported. The modifications of the protein structure and the formation of a complex with the GNMs are demonstrated by UV-Vis absorption and static and time-resolved fluorescence data. Atomic force microscopy (AFM) is used as complementary tool to characterize the protein-GNM complex.

\section{Design of plasmonic GNMs}

\subsection{Nanorods}

A class of GNMs, which has attracted much attention because of their potential as therapeutic agents in biomedicine, is gold nanorods (NRDs). They present two LSPR extinction bands, which correspond to the oscillation of the plasmons in the transversal and the longitudinal direction. The transversal mode is not strongly affected by the NRD morphology and the relative absorption occurs at about $520 \mathrm{~nm}$. On the contrary, the position of the longitudinal LSPR can shift from the visible region to the NIR depending on the aspect ratio (length-towidth ratio). Higher is the aspect ratio, lower is the frequency at which the plasmon absorbs. In order to design NRDs with the desired optical properties, several computational methods have been developed to correlate the morphology of the NRDs with their absorption and scattering efficiencies [16-18] obtaining in most cases an excellent agreement between the simulations and the experimental data.

The first report of the synthesis of gold NRDs is from the group of Jana et al. in 2001 [19]. This experimental procedure is known as the seed-mediated growth method and it is still the most common preparation for this type of GNM. In this synthesis, gold nanoparticles of 1-3 nm (seeds) are obtained by reduction of a $\mathrm{Au}^{3+}$ salt by $\mathrm{NaBH}_{4}$ in the presence of a surfactant, usually sodium citrate or cetyltrimethylammonium bromide (CTAB), as stabilizer. These seeds are then quickly added to an aqueous growth solution containing $\mathrm{AgNO}_{3}, \mathrm{CTAB}$ and $\mathrm{Au}^{+}$ions formed by partial reduction of $\mathrm{Au}^{3+}$ with ascorbic acid. The seeds act as nucleation centers for the reduction of $\mathrm{Au}^{+}$to atomic gold and $\mathrm{CTAB}$, which has a strong affinity for specific facets of the metal, promotes the anisotropic growth of the rod. Through this method, it is possible to obtain NRDs of about $15 \mathrm{~nm}$ wide and up to $100 \mathrm{~nm}$ long depending on the amount of $\mathrm{AgNO}_{3}$ and the seed to gold molar ratio in the growth solution. NRDs with higher aspect ratio 
presenting the longitudinal LSPR region can be still produced with this procedure by an additional growth step in the presence of a mixture of two surfactants [20].

The extinction spectra and the corresponding transmission electron microscopy (TEM) images of two samples of NRDs synthesized by our group using the seed-mediated growth method are shown in Figure 1. The sample (a), having an aspect ratio of $2.3 \pm 0.2$ as determined by the analysis of the TEM images, presents an intense longitudinal LSPR absorption band centered at $652 \mathrm{~nm}$. The use of a larger amount of silver ions in the growth solution increases the NRDs aspect ratio to $4.8 \pm 0.2$, which shifts the longitudinal band to $775 \mathrm{~nm}$ (Figure 1, sample b).

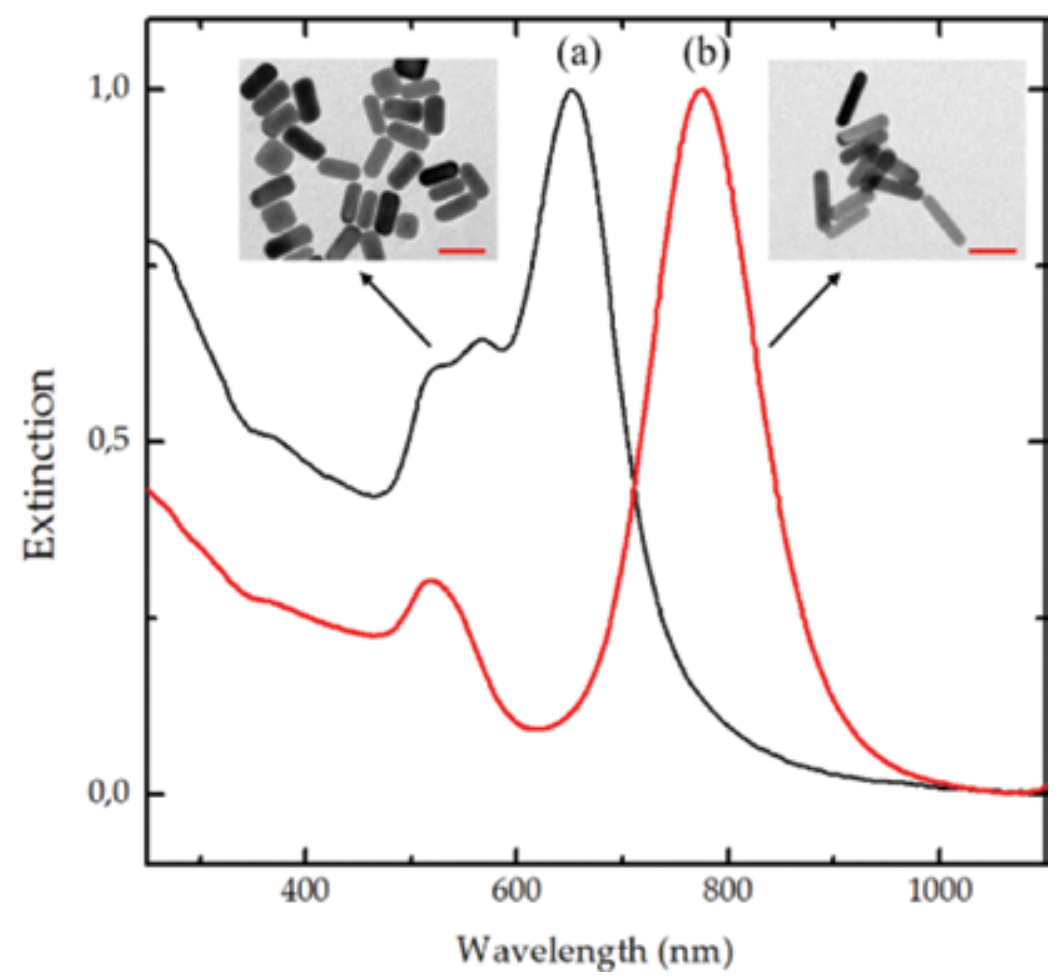

Figure 1. Extinction spectra of NRDs with an aspect ratio of 2.3 (a) and 4.8 (b) and the corresponding TEM images $($ scale bar $=50 \mathrm{~nm})$. An increase in the amount of silver ions added in solution allows the formation of nanorods with a higher aspect ratio and with an extinction spectra shifted at longer wavelengths.

\subsection{Nanoshells}

An interesting approach to shift the LSPR absorption in the NIR region is the synthesis of gold nanoshells (NSHs) that are hybrid nanostructures formed by a dielectric core (such as silicon dioxide) covered by a thin and uniform layer of gold. Their optical properties have been rationalized in terms of the coupling between the plasmons of the internal and the external metal surface; the plasmon hybridization model, which can be seen as the analog of the molecular orbital theory for molecules, demonstrates that the extent of the coupling, and thus 
the shift toward longer wavelengths of the LSPR, depends on the shell/core size ratio [21, 22]. These calculations are in good agreement with the experimental data.

Brinson et al. [23] in 2008 first developed a reliable multistep approach to prepare the gold NHSs. The synthesis starts from $\mathrm{SiO}_{2}$ nanoparticles formed through the sol-gel method [24] and whose surface is functionalized with amino groups. Separately, gold seeds of $2-5 \mathrm{~nm}$ in diameter are prepared by reduction of a gold salt with an organophosphorus compound in water and then adsorbed on the amino-functionalized silica nanoparticles. With a process similar to the one described earlier for the NRDs, the further reduction of $\mathrm{Au}^{3+}$ by formaldehyde on the seed attached to the $\mathrm{SiO}_{2}$ surface allows the growth of a complete gold shell. The thickness of the metal layer and thus the position of the LSPR absorption band can be controlled by changing the amount of gold salt in the growth solution. In Figure 2, the extinction spectra of NSHs synthesized in our laboratory together with the corresponding TEM images are shown. Amino-functionalized $\mathrm{SiO}_{2}$ nanoparticles of $120 \mathrm{~nm}$ were used as template for the formation of the shells. From the analysis of the TEM images, a thickness of the gold layer of $11 \pm 1 \mathrm{~nm}$ has been measured for sample (b), which corresponded to a LSPR absorption band centered at $805 \mathrm{~nm}$. When a higher concentration of gold salt was present in the growth solution, the shell thickness was increased to $20 \pm 1 \mathrm{~nm}$, which resulted in a surface plasmon band blue shifted to 550-600 $\mathrm{nm}$ (Figure 2a). This is explained by the increase in the distance between the inner and the outer metal layer that causes uncoupling of the two surfaces and the extinction spectrum resembles one of the clustered gold nanoparticles on silica [25]. This example illustrates the importance to achieve a strict control of the synthesis parameters in order to obtain nanoparticles with the desired properties.

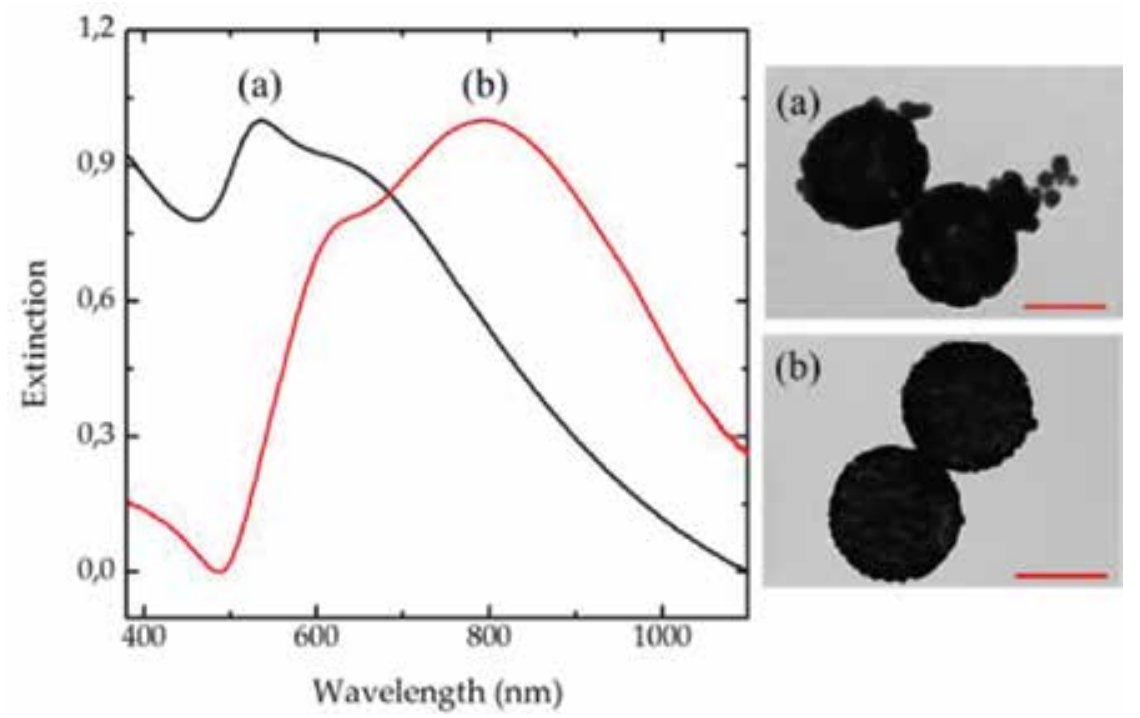

Figure 2. Extinction spectra of NSHs prepared with a $\left[\mathrm{Au}^{3+}\right]$ concentration in solution of (a) $39.2 \mu \mathrm{M}$ and (b) $32.4 \mu \mathrm{M}$ and the corresponding TEM images (scale bar $=100 \mathrm{~nm}$ ). The amount of gold ions in solution determines the metal shell thickness and hence the position of the SPR band of the NSHs. 
Recently, this multistep approach has been adapted by our group to obtain NSHs with a fluorescent core for biomedical applications [26].

\subsection{Strategies for the surface functionalization of GNMs}

The functionalization of the GNMs surface is often required for the following reasons: replacing stabilizers used for the synthesis that are toxic for the cells [27, 28], enhancing their stability in biological environments $[29,30]$ and introducing species (e.g., antibodies) to promote site-specific delivery of the system in the body [31,32]. When the stabilization of the GNMs has an electrostatic nature (as for example for citrate-stabilized gold nanoparticles prepared by the common Turkevitch method [33]), the ligands can be easily substituted by stronger thiolated stabilizers under mild conditions. Using the suitable thiol linker, it is possible to functionalize the GNMs with a wide range of different species such as polymers [34, 35], fluorophores [36], deoxyribonucleic acid (DNA) [37], and antibodies [38, 39]. The ligand exchange is generally carried out at room temperature and it can also involve the use of a biphasic system [40]. However, this method has also some drawbacks. For example, the replacement of the stabilizer could be incomplete making difficult to determine the exact amount of the ligand exchanged. Also, the exchange process could lead to irreversible aggregation.

It has been shown that CTAB is toxic to the cells [41] and CTAB-coated NRDs are unstable in basic conditions [42]. However, its replacement from the surface of the gold nanorods is particularly tricky. The use of thiolate ligands usually brings to a partial exchange on the ends of the NRDs because of the preferential binding of these species on the $\mathrm{Au}\{111\}$ facets [43]. In recent years, various methods have been developed to obtain a complete CTAB removal avoiding aggregation. One of the approaches that can be used is the layer-by-layer deposition of polyelectrolytes on the CTAB-coated NRDs, which assures the control of the polymeric shell and the final surface charge of the rods. This method has been used with good results by several groups [42, 44, 45]. However, the main disadvantage is that the further functionalization of the surface with site-specific ligands is not easy to obtain. The growth of a silica shell around the nanorods is another strategy that can be adopted to remove the surfactant from the metal surface. $\mathrm{SiO}_{2}$ is a good choice because it is a biocompatible material, it can be prepared with low cost and reliable methods and it can be easily functionalizable with other specific ligands. The silica coating can be carried out in a simple single-step process following the procedure first reported by Gorelikov et al. [46]. Briefly, CTAB-capped NRDs are diluted in water and $\mathrm{NaOH}$ is added to raise the $\mathrm{pH}$ to $\sim 10$. Then, the sequential addition of tetraethylorthosilicate (TEOS) as silica precursor to this solution allows the hydrolysis and condensation of the shell on the NRD surface. After mixing overnight, the silica-coated NRDs are separated from CTAB by centrifugation, removal of the supernatant and redispersion in the fresh solvent. The careful control of the TEOS-to-NRDs molar ratio in the reaction mixture and the amount of TEOS chosen for each addition determines the success of the procedure and the thickness of the coating. To demonstrate this with an example, Figure 3 shows the extinction spectra and the TEM images of two samples of silica-coated NRDs with an aspect ratio of 2.3 prepared by our group in different experimental conditions. 

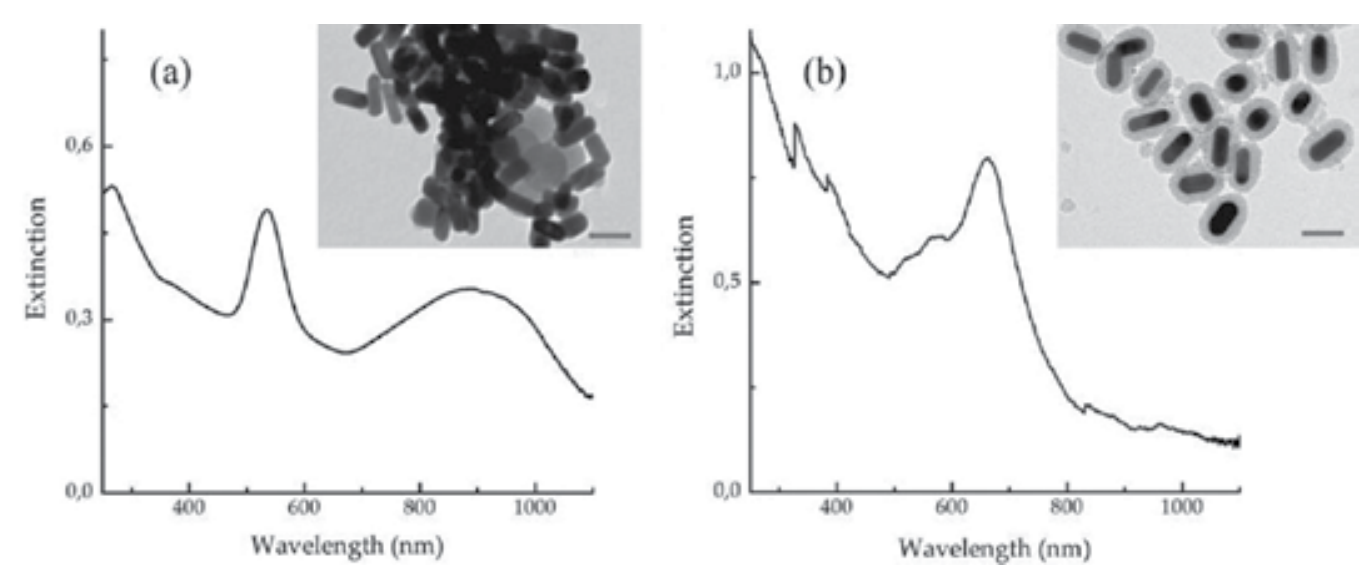

Figure 3. Extinction spectra of silica-coated NRDs prepared with a TEOS concentration in the solution of (a) $10.0 \mathrm{mM}$ and (b) $2.3 \mathrm{mM}$ together with the corresponding TEM images (scale bar $=50 \mathrm{~nm}$ ). The growth of a homogenous silica shell is obtained by choosing the appropriate TEOS-to-NRDs molar ratio in the reaction mixture. An increase in TEOS content in solution leads to irreversible aggregation of the NRDS.

For sample (a), the coating process resulted in the aggregation of the nanorods. This aggregation is also confirmed by the extinction spectrum, which shows a broad and unstructured band with a maximum at about $900 \mathrm{~nm}$ that is red shifted with respect to the one of the initial CTABcoated NRDs. For sample (b), the fine control of the experiment allowed us to obtain a homogeneous coating of the nanorods with a silica shell of $16 \pm 1 \mathrm{~nm}$. In this case, the extinction spectrum resembles that of the NRDs before the exchange of the stabilizer. This example also proves that the optical properties of the plasmonic nanoparticles can be successfully used to monitor the stability of the system in a fast and reliable manner during postsynthesis treatments.

\section{Interaction of GNMs with proteins}

Myoglobin (Mb) and bovine serum albumin (BSA) are used as model proteins to study the interaction with GNMs. The structure of Mb consists of a single polypeptide chain of 153 amino acids, which is arranged in eight $\alpha$-helices for about the $75 \%$ of its length. The fluorescence of $\mathrm{Mb}$ is dominated by the emission of its two Trp residues with one of them located at the protein surface (Trp-7) and the other one buried inside the hydrophobic pocket of protein (Trp-14). However, this emission is partially quenched by energy or electron transfer to the heme group in the protein [47] and this explains the low fluorescence quantum yield of $\mathrm{Mb}$ in the native form. BSA is a relatively bigger protein (583 amino acids) that has numerous biochemical functions and the most important function is the transport of drugs and fatty acids inside the body [48-50]. In analogy with $\mathrm{Mb}$, it contains two Trp residues, one on the surface (Trp-134) and the other in a hydrophobic domain (Trp-212), which are responsible for the intrinsic fluorescence of the protein [51]. Modifications of the fluorescence properties of the proteins offer useful information on structural changes, rearrangements and eventually denaturation 
processes occurring upon interaction with different types of chemical species [52-56]. In the following section, the same techniques have been applied to the study of GNMs-protein association.

\subsection{Interaction of $\mathrm{Mb}$ with GNMs}

NSHs with a silica diameter of $120 \mathrm{~nm}$ and a gold shell thickness of $11 \mathrm{~nm}$ (for morphological and optical properties refer to Figure 2) were gradually added to a $10^{-5} \mathrm{M}$ aqueous solution of $\mathrm{Mb}$. As shown in Figure 4a, the fluorescence spectrum of the protein drastically changed by interaction with the NSHs. A decrease of the band at $320 \mathrm{~nm}$ associated with the Trp fluorescence and the appearance of two additional emission bands centered at 390 and $440 \mathrm{~nm}$ were observed. Different excitation spectra were recorded at 330 and $440 \mathrm{~nm}$ (Figure 4b), demonstrating that upon interaction with NSHs two distinct emitting species become responsible of the $\mathrm{Mb}$ fluorescence. Time-resolved data reported in Table 1 further supported this hypothesis. The fluorescence decay curve recorded at $330 \mathrm{~nm}$ for the native protein was successfully fitted with a monoexponential function giving a lifetime value of $2.6 \mathrm{~ns}$. When NSHs were added to the solution of $\mathrm{Mb}$, this value was reduced of about $20 \%$.
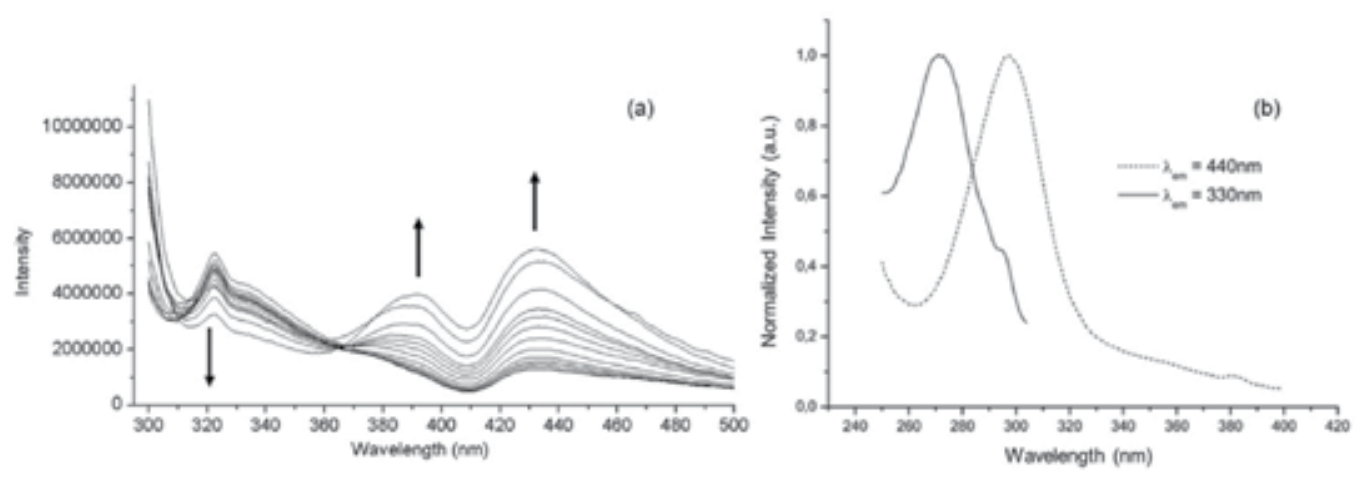

Figure 4. (a) Fluorescence emission spectra of $\mathrm{Mb}\left(\lambda_{\text {exc }}=290 \mathrm{~nm}\right)$ in the presence of increasing concentration of NSHs; (b) fluorescence excitation spectra of $\mathrm{Mb}$ in the presence of $2.5 \times 10^{-12} \mathrm{M}$ NSHs recorded at two emission wavelengths. The fluorescence data indicate that a protein-nanoparticle complex is formed upon interaction of $\mathrm{Mb}$ with the NSHs.

The fluorescence decay signal at 390 and $440 \mathrm{~nm}$ showed a more complex behavior, and from the analysis of the data, we obtained two decay components of about 4.5 and 1.0-1.5 ns. These values and their relative statistical weight on the lifetime were not affected by the concentration of nanoshells in solution. All the fluorescence data suggested that the interaction of $\mathrm{Mb}$ with the NSHs brings to the formation of a fluorescent protein-nanoparticle complex in which the protein is in a different conformational arrangement or is partially denaturated. The value of the longer and principal component (4.5 ns) of the decay of the new species was close to the value of Trp lifetime in water [57], which would indicate that in the rearranged configuration the two Trp are more exposed to the external medium. However, the decrease in the lifetime value at $330 \mathrm{~nm}$ suggests that static quenching due to the formation of the $\mathrm{Mb}-\mathrm{NSH}$ complex 
alone cannot explain the mechanism of interaction, and energy/electron transfer processes between the Trp residues and the gold are not excluded.

\begin{tabular}{llll}
\hline [NSHs] (M) & $\lambda_{\text {em }}(\mathbf{n m})$ & $\tau(\mathbf{n s})$ & Percentage $(\%)$ \\
\hline 0 & 330 & 2.6 & 100 \\
$7.2 \times 10^{-13}$ & 330 & 2.1 & 100 \\
& 390 & 4.6 & 71.3 \\
& & 0.9 & 28.7 \\
$2.5 \times 10^{-12}$ & 440 & 4.5 & 94.3 \\
& 330 & 1.5 & 5.7 \\
& 390 & 2.1 & 100 \\
& 4.5 & 80.5 \\
& 440 & 0.9 & 19.5 \\
& 4.4 & 98.0 \\
& & 1.1 & 2.0
\end{tabular}

Table 1. Fluorescence decay time parameters of $\mathrm{Mb}\left(\lambda_{\mathrm{exc}}=295 \mathrm{~nm}\right)$ in the presence of increasing concentration of NSHs.

The same experiment has been carried out by using CTAB-capped gold nanorods with an aspect ratio of 2.3 (Figure 1a for the optical and microscopy data of the sample). The addition of $\mathrm{NRDs}$ to a $10^{-5} \mathrm{M}$ solution of $\mathrm{Mb}$ in water efficiently quenched the protein emission, but in this case, no additional bands at longer wavelengths were detected (Figure 5a).
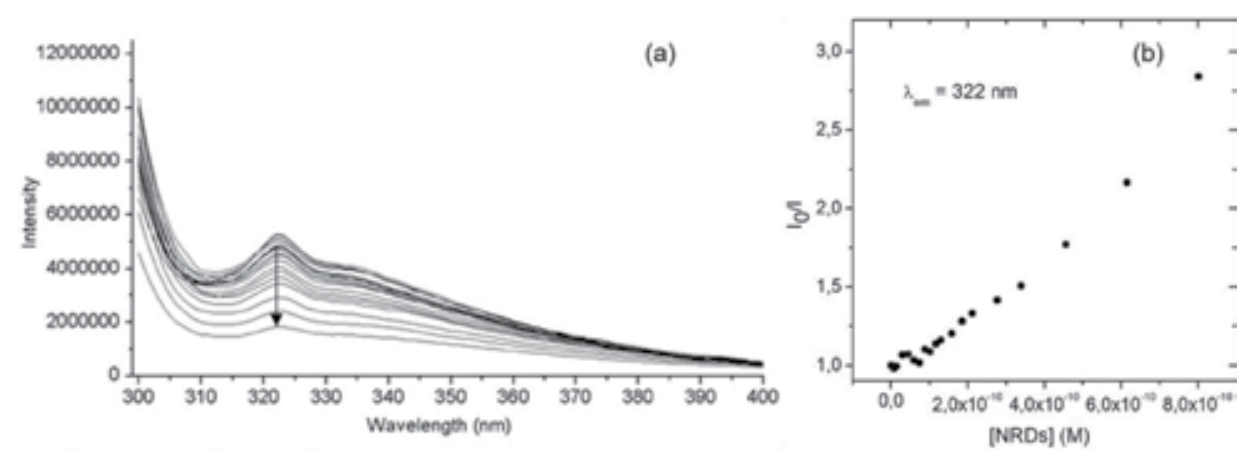

Figure 5. (a) Fluorescence emission spectra of $\mathrm{Mb}\left(\lambda_{\text {exc }}=290 \mathrm{~nm}\right)$ in the presence of increasing concentration of NRDs; (b) Stern-Volmer plot from the emission intensity values at $322 \mathrm{~nm}$. The efficient quenching of the fluorescence observed upon addition of NRDs to the $\mathrm{Mb}$ solution demonstrates that a protein-nanoparticle complex in the ground state is formed. 
One of the most used models to analyze fluorescence data is through the Stern-Volmer equation, which can give indications about the occurrence of both static and dynamic quenching processes:

$$
\frac{I_{0}}{I}=1+K_{S V}[Q]=1+k_{q} \tau_{0}[Q]
$$

where $I_{o}$ and I are the fluorescence intensities in the absence and in the presence of the quencher, $\mathrm{K}_{\mathrm{sV}}$ is the Stern-Volmer quenching constant, $\mathrm{k}_{\mathrm{q}}$ is the bimolecular quenching rate constant, $\tau_{0}$ is the average lifetime of the fluorophore in the absence of the quencher and [Q] is the concentration of the quencher in solution. The Stern-Volmer plot of fluorescence data (Figure 5b) showed a linear relationship and a $\mathrm{K}_{\mathrm{Sv}}$ value of $1.9 \times 10^{9} \mathrm{M}^{-1}$ was obtained from the linear fit. Using $2.6 \mathrm{~ns}$ for the lifetime of the myoglobin (Table 1), we estimated a $\mathrm{k}_{\mathrm{q}}$ of 7.3 $\times 10^{17} \mathrm{M}^{-1} \mathrm{~s}^{-1}$ that is several orders of magnitude greater than the maximum dynamic collisional quenching constants of various types of quenchers with biomolecules [58]. This demonstrates that the quenching has an important static component and that a nonfluorescent $\mathrm{Mb}-\mathrm{NRD}$ complex in the ground state is formed. The fluorescence measurements clearly indicate that the interaction between GNMs and the myoglobin occurs in any case through the adsorption of the proteins on the surface of the nanoparticle although with a different structural rearrangement. Different surface properties of the GNMs tuned by their size and by the nature of the stabilizers could explain these differences.

The role of the surface properties of the plasmonic NPs in the interaction with the proteins has been further investigated by using NRDs with the same aspect ratio of 2.3 in which the surfactant $\mathrm{CTAB}$ has been replaced by a uniform silica shell of $16 \pm 1 \mathrm{~nm}$ (Figure $3 \mathbf{b}$ for the optical and morphological data of these nanoparticles).

The addition of silica-coated NRD to a $10^{-5} \mathrm{M}$ solution of $\mathrm{Mb}$ brought to drastic changes in the fluorescence spectrum of the protein (Figure 6). The complete quenching of the emission of the native protein and the appearance of a new broad band at $365 \mathrm{~nm}$ was detected right after the addition of the nanoparticles. This new emission can be attributed to Trp residues that are completely exposed to a polar environment $[49,59]$. The data indicate that the interaction of $\mathrm{Mb}$ with the NRD surface is followed by the denaturation of the protein that brings the Trp-14 to be in contact with the water solvent. The adsorption of $\mathrm{Mb}$ on the silica-coated nanorods has been also demonstrated by AFM microscopy. A drop of the solution containing the protein and the silica coated NRD at concentrations of $10^{-5} \mathrm{M}$ and $5 \times 10^{-12} \mathrm{M}$, respectively, was deposited on freshly cleaved mica. After complete evaporation of the solvent, the sample was scanned with an AFM in tapping mode. The topographic image in Figure 7a shows objects of about 200-250 nm that can be associated with aggregates of 2-3 nanoparticles. The irregular surface of these particles and the presence of regions at different phase contrast (Figure $7 \mathbf{b}$ ) indicate that these species are covered by $\mathrm{Mb}$ molecules as previously observed with microscopy techniques for other protein-silica systems studied by our group. The presence of protein molecules on the surface of the silica-coated NRDs could also assist and promote the aggregation process [60]. 


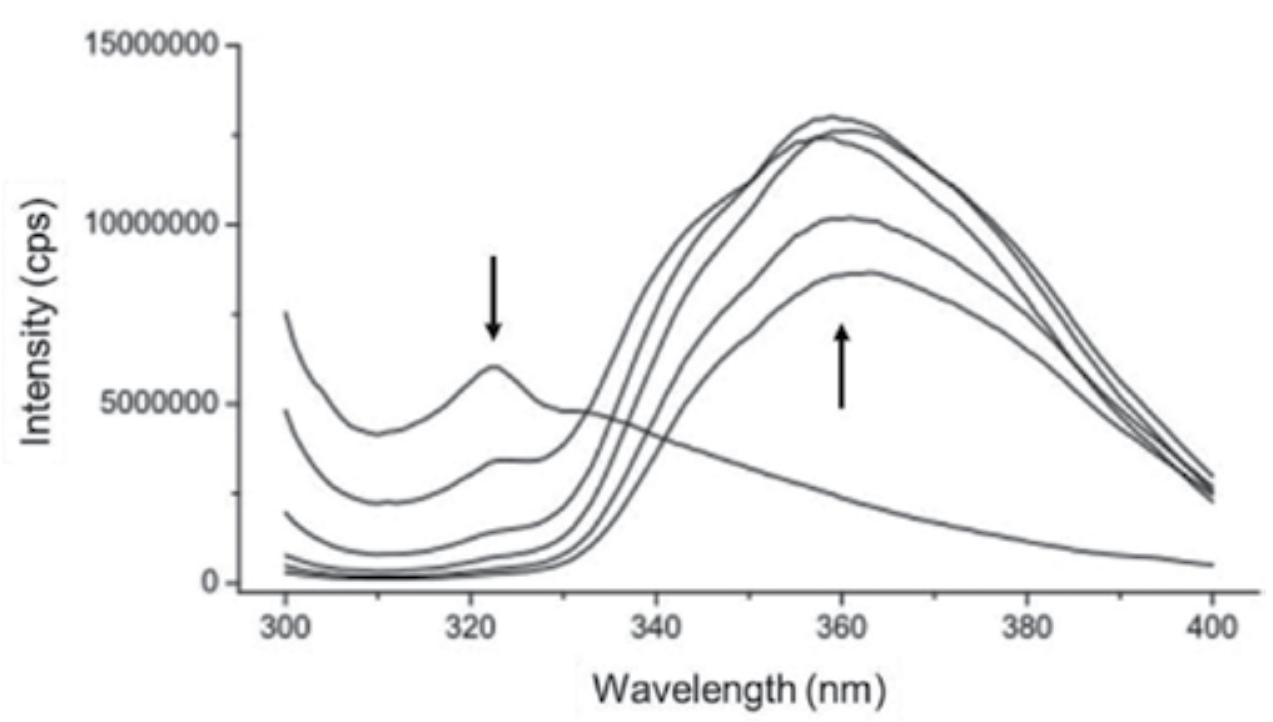

Figure 6. Fluorescence emission spectra of $\mathrm{Mb}\left(\lambda_{\mathrm{exc}}=290 \mathrm{~nm}\right)$ in the presence of increasing concentration of silica-coated NRDs (from 0 to $6.8 \times 10^{-12} \mathrm{M}$ ). The appearance of a new emission band at $365 \mathrm{~nm}$ is attributed to the exposure of $\operatorname{Trp}$ residues of $\mathrm{Mb}$ to the aqueous environment following the protein denaturation.
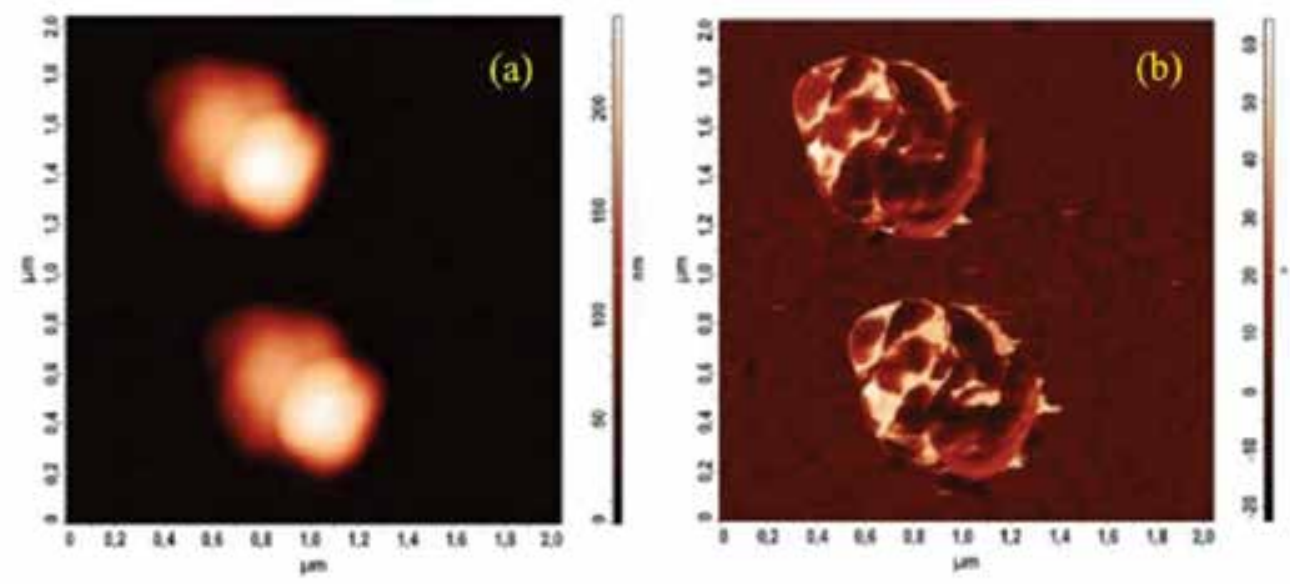

Figure 7. (a) Topographic and (b) phase-contrast AFM image of myoglobin and silica-coated NRDs after deposition on mica substrate. Aggregates of nanoparticles covered by myoglobin molecules are clearly observed from the images.

\subsection{Interaction of BSA with GNMs}

NRDs with different surface properties have been employed to study the interactions of plasmonic nanoparticles with BSA. The fluorescence spectra of the protein upon gradual addition of increasing amount of nanorods with an aspect ratio of 2.3 are reported in Figure 8 . 


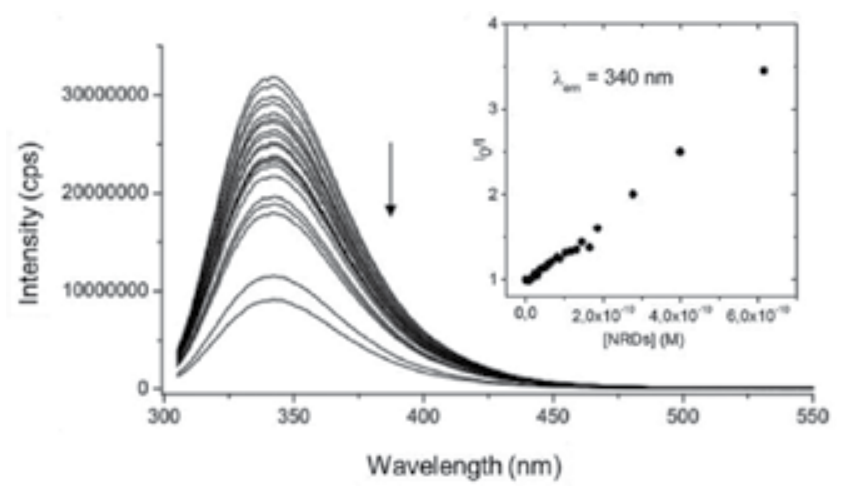

Figure 8. Fluorescence emission spectra of BSA $\left(\lambda_{\mathrm{exc}}=290 \mathrm{~nm}\right)$ in the presence of increasing concentration of NRDs; (inset) Stern-Volmer plot from the emission intensity values at $340 \mathrm{~nm}$. The strong quenching of the BSA emission demonstrates the formation of nonfluorescent protein-NRD complexes.

Analogous to $\mathrm{Mb}$, an efficient quenching of fluorescence was observed without any modification in the shape of the emission band. The intensity values treated with the Stern-Volmer equation showed a linear correlation of the quenching with the concentration of the NRDs added. The analysis allowed to calculate a $\mathrm{K}_{\mathrm{SV}}$ value of $3.9 \times 10^{9} \mathrm{M}^{-1}$ and a $\mathrm{k}_{\mathrm{q}}$ of $8.1 \times 10^{17} \mathrm{M}^{-1}$ $\mathrm{cm}^{-1}$ using a calculated average lifetime of $4.8 \mathrm{~ns}$ for BSA. These values demonstrate once again that the interaction is dominated by static quenching, and thus, a nonfluorescent GNM-protein complex at the ground state is formed.

When a silica shell of $16 \mathrm{~nm}$ was grown on the NRDs replacing the surfactant, the interaction with the BSA molecules changed dramatically. In fact, as shown in Figure 9a, a quenching much more efficient was detected even at lower concentrations of silica-coated NRDs in

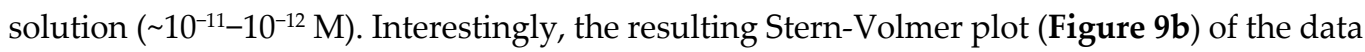

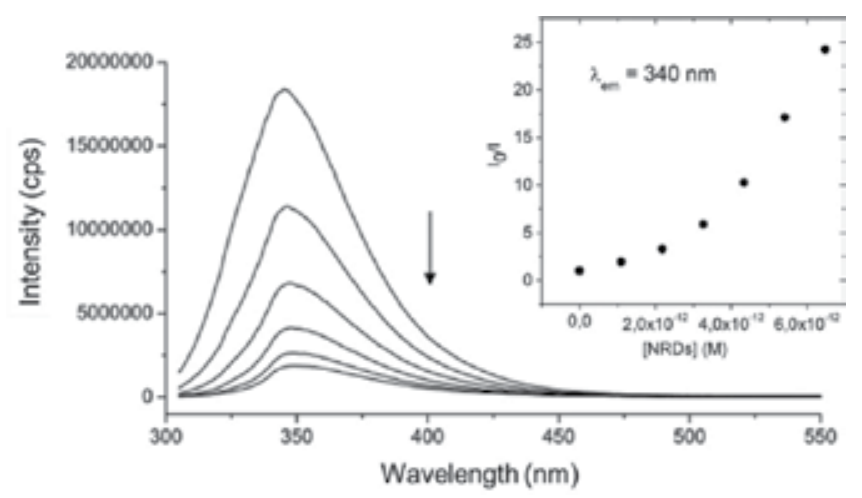

Figure 9. Fluorescence emission spectra of BSA $\left(\lambda_{\text {exc }}=290 \mathrm{~nm}\right)$ in the presence of increasing concentration of silicacoated NRDs; (inset) Stern-Volmer plot from the emission intensity values at $340 \mathrm{~nm}$. A more complex mechanism of interaction occurs when a silica shell is grown around the nanorods interacting with the BSA molecules. This results in a more efficient quenching and an upward curvature of the Stern-Volmer plot. 
showed an upward curvature which indicates that the decrease in the fluorescence intensity might be due to a bimodal mechanism of interaction. A small red shift of $2-3$ of the emission maximum also suggests that a certain rearrangement of BSA follows the adsorption of the protein on the silica surface of the nanorods.

\section{Conclusion}

Nowadays, several nanodevice families are available as biomedical tools. Among them, goldbased nanomaterials have a prominent role to play because of their excellent properties. In this chapter, we demonstrated that it is possible to obtain GNMs through simple and reliable chemical methods and their optical properties can be tuned by changing the synthetic conditions. Nanorods capped with CTAB can be prepared by a seed-mediated growth method in aqueous solution and the final aspect ratio strongly affects the position of the longitudinal plasmon band of the extinction spectrum. The removal of the toxic CTAB from the metal surface of the rods is necessary in order to use these them for in vivo applications and we showed that different approaches can be used to exchange this surfactant with other stabilizers. By a fine control of the preparation conditions, it is possible to grow a silica shell of variable thickness on the nanorods, which can be further functionalized with site-specific ligands. Another interesting system showing plasmonic properties is gold nanoshell made of a silica core covered by a thin layer of gold. Depending on the ratio between the size of the core and the shell thickness, the surface plasmon absorption can be shifted from the visible to the NIR region of the spectrum which is desirable for biomedical applications.

In the second section, we described in detail the interaction of model proteins with these two types of gold-based nanomaterials through fluorescence and microscopy techniques. In all cases, quenching of the protein emission was detected. The data analyzed by the Stern-Volmer equation indicate that protein-nanoparticles complexes are formed at the ground state. The appearance of new red-shifted emission bands in the fluorescence spectra and the AFM images confirmed the presence of these species. The comparison between the data collected for the nanorods before and after growth of a silica shell demonstrated that the surface properties of the nanoparticles have an important role in regulating the process of protein adsorption.

The use of GNMs as biomedical devices requires the in-depth study of their therapeutic efficacy along with their potential toxicity. In this context, a deep comprehension on how the biological molecules interact with the nanoparticles is desirable. Several recent studies have been focused on the biological effects of GNMs, but a detailed description of the mechanism of interaction between proteins and nanoparticles and a comprehensive study of the factors affecting this interaction is still lacking. This knowledge would bring enormous advantages in the design of nanomaterials with controllable properties. The development of methods sensitive enough for the detailed study of NP-proteins interaction is also a challenge. For this purpose, singlemolecule techniques and ultrafast spectroscopy may play a fundamental role in elucidating the mechanism of interaction between a protein and a metal surface with high spatial resolution. Moreover, a multimethod approach would allow describing in detail the process of 
protein adsorption onto GNMs from different points of view. The big question that remains open is how to obtain the interaction information with a similar sensitivity in a living organism. By addressing these issues, it will be possible to create gold-based nanostructures with engineered surfaces that could interact predictably with proteins opening up the possibility to obtain more efficient therapeutic agents and nanoprobes for biomedical applications.

\section{Author details}

Loredana Latterini* and Luigi Tarpani

*Address all correspondence to: loredana.latterini@unipg.it

Department of Chemistry, Biology and Biotechnology, Centro Eccellenza Materiali Innovativi Nanostrutturati (CEMIN), University of Perugia, Italy

\section{References}

[1] Grassian VH. When size really matters: size-dependent properties and surface chemistry of metal and metal oxide nanoparticles in gas and liquid phase environment. J Phys Chem C. 2008;112(47):18303-13. DOI: 10.1021/jp806073t

[2] El-Sayed MA. Small is different: shape-, size-, and composition-dependent properties of some colloidal semiconductor nanocrystals. Acc Chem Res. 2004;37(5):326-33. DOI: 10.1021/ar020204f

[3] Deng ZJ, Liang M, Monteiro M, Toth I, Minchin RF. Nanoparticle-induced unfolding of fibrinogen promotes Mac-1 receptor activation and inflammation. Nature Nanotech. 2011;6(1):39-44. DOI: 10.1038/nnano.2010.250

[4] Simón-Vázquez R, Lozano-Fernández T, Peleteiro-Olmedo M, González-Fernández Á. Conformational changes in human plasma proteins induced by metal oxide nanoparticles. Coll Surf B: Bioint. 2014;113:198-206. DOI: 10.1016/j.colsurfb.2013.08.047

[5] Tsai YS, Chen YH, Cheng PC, Tsai HT, Shiau AL, Tzai TS, Wu CL. TGF- $\beta 1$ Conjugated to gold nanoparticles results in protein conformational changes and attenuates the biological function. Small. 2013;9(12):2119-28. DOI: 10.1002/smll.201202755

[6] Saha K, Agasti SS, Kim C, Li X, Rotello V M. Gold nanoparticles in chemical and biological sensing. Chem Rev. 2012;112(5);2739-79. DOI: 10.1002/anie.200603735

[7] Choi $\mathrm{Y}$, Ho NH, Tung $\mathrm{CH}$. Sensing phosphatase activity by using gold nanoparticles. Angew Chem Int Ed. 2007;46(5):707-9. 
[8] De M, Rana S, Akpinar H, Miranda OR, Arvizo RR, Bunz UH, Rotello VM. Sensing of proteins in human serum using conjugates of nanoparticles and green fluorescent protein. Nat Chem. 2009;1(6):461-5. DOI: 10.1038/nchem.334

[9] Popovtzer R, Agrawal A, Kotov NA, Popovtzer A, Balter J, Carey TE, Kopelman R. Targeted gold nanoparticles enable molecular CT imaging of cancer. Nano Lett. 2008;8(12):4593-96. DOI: 10.1021/n18029114

[10] Jain PK, Lee KS, El-Sayed IH, El-Sayed MA. Calculated absorption and scattering properties of gold nanoparticles of different size, shape, and composition: applications in biological imaging and biomedicine. J Phys Chem B. 2006;110(14):7238-48. DOI: 10.1021/jp057170o

[11] Li D, Teoh WY, Selomulya C, Woodward RC, Amal R, Rosche B. Flame-sprayed superparamagnetic bare and silica-coated maghemite nanoparticles: synthesis, characterization, and protein adsorption-desorption. Chem Mater. 2006;18(26):640313. DOI: $10.1021 / \mathrm{cm} 061861 \mathrm{v}$

[12] Lehman SE, Mudunkotuwa IA, Grassian VH, Larsen SC. Nano-bio interactions of porous and nonporous silica nanoparticles of varied surface chemistry: a structural, kinetic, and thermodynamic study of protein adsorption from RPMI culture medium. Langmuir. 2015;32(3):731-42. DOI: 10.1021/acs.langmuir.5b03997

[13] Calzolai L, Franchini F, Gilliland D, Rossi F. Protein-nanoparticle interaction: identification of the ubiquitin-gold nanoparticle interaction site. Nano Lett. 2010;10(8):31015. DOI: $10.1021 / \mathrm{nl} 101746 \mathrm{v}$

[14] Lundqvist M, Sethson I, Jonsson BH. Protein adsorption onto silica nanoparticles: conformational changes depend on the particles' curvature and the protein stability. Langmuir. 2004;20(24):10639-47. DOI: 10.1021/la0484725

[15] Cedervall T, Lynch I, Lindman S, Berggård T, Thulin E, Nilsson H, Dawson KA, Linse S. Understanding the nanoparticle-protein corona using methods to quantify exchange rates and affinities of proteins for nanoparticles. Proc Natl Acad Sci. 2007;104(7):20505. DOI: $10.1073 /$ pnas.0608582104

[16] Link S, Mohamed M, El-Sayed M. Simulation of the optical absorption spectra of gold nanorods as a function of their aspect ratio and the effect of the medium dielectric constant. J Phys Chem B. 1999;103(16):3073-7. DOI: 10.1021/jp990183f

[17] Brioude A, Jiang X, Pileni M. Optical properties of gold nanorods: DDA simulations supported by experiments. J Phys Chem B. 2005;109(27):13138-42. DOI: 10.1021/ jp0507288

[18] Ni W, Kou X, Yang Z, Wang J. Tailoring longitudinal surface plasmon wavelengths, scattering and absorption cross sections of gold nanorods. ACS Nano. 2008;2(4):67786. DOI: $10.1021 /$ nn7003603 
[19] Jana NR, Gearheart L, Murphy CJ. Wet chemical synthesis of high aspect ratio cylindrical gold nanorods. J Phys Chem B. 2001;105(19):4065-7. DOI: 10.1021/jp0107964

[20] Nikoobakht B, El-Sayed MA. Preparation and growth mechanism of gold nanorods (NRs) using seed-mediated growth method. Chem Mater. 2003;15(10):1957-62. DOI: 10.1021/cm0207321

[21] Prodan E, Radloff C, Halas NJ, Nordlander P. A hybridization model for the plasmon response of complex nanostructures. Science. 2003;302(5644):419-22. DOI: 10.1126/ science.1089171

[22] Jain PK, El-Sayed MA. Universal scaling of plasmon coupling in metal nanostructures: extension from particle pairs to nanoshells. Nano Lett. 2007;7(9):2854-8. DOI: 10.1021/ nl071496m

[23] Brinson BE, Lassiter JB, Levin CS, Bardhan R, Mirin N, Halas NJ. Nanoshells made easy: improving Au layer growth on nanoparticle surfaces. Langmuir. 2008;24(24):14166-71. DOI: $10.1021 / 1 \mathrm{la} 802049 \mathrm{p}$

[24] Stöber W, Fink A, Bohn E. Controlled growth of monodisperse silica spheres in the micron size range. J Coll Interf Sci. 1968;26(1):62-9. DOI: 10.1016/0021-9797(68)90272-5

[25] Westcott SL, Oldenburg SJ, Lee TR, Halas NJ. Construction of simple gold nanoparticle aggregates with controlled plasmon-plasmon interactions. Chem Phys Lett. 1999;300(5):651-5. DOI: 10.1016/S0009-2614(98)01410-9

[26] Latterini L, Tarpani L. Hierarchical assembly of nanostructures to decouple fluorescence and photothermal effect. J Phys Chem C. 2011;115(43):21098-104. DOI: 10.1021/ jp208124x

[27] Alkilany AM, Nagaria PK, Hexel CR, Shaw TJ, Murphy CJ, Wyatt MD. Cellular uptake and cytotoxicity of gold nanorods: molecular origin of cytotoxicity and surface effects. Small. 2009;5(6):701-8. DOI: 10.1002/smll.200801546

[28] Rayavarapu RG, Petersen W, Hartsuiker L, Chin P, Janssen H, Van Leeuwen FW, Otto C, Manohar S, Van Leeuwen TG. In vitro toxicity studies of polymer-coated gold nanorods. Nanotechnology. 2010;21(14):145101. DOI: 10.1088/0957-4484/21/14/145101

[29] Chompoosor A, Han G, Rotello VM. Charge dependence of ligand release and monolayer stability of gold nanoparticles by biogenic thiols. Bioconj Chem. 2008;19(7):134245. DOI: $10.1021 / \mathrm{bc} 8000694$

[30] Rosi NL, Giljohann DA, Thaxton CS, Lytton-Jean AK, Han MS, Mirkin CA. Oligonucleotide-modified gold nanoparticles for intracellular gene regulation. Science. 2006;312(5776):1027-30. DOI: 10.1126/science.1125559

[31] Leduc C, Si S, Gautier J, Soto-Ribeiro M, Wehrle-Haller B, Gautreau A, Giannone G, Cognet L, Lounis B. A highly specific gold nanoprobe for live-cell single-molecule imaging. Nano Lett. 2013;13(4):1489-94. DOI: 10.1021/nl304561g 
[32] Black KC, Yi J, Rivera JG, Zelasko-Leon DC, Messersmith PB. Polydopamine-enabled surface functionalization of gold nanorods for cancer cell-targeted imaging and photothermal therapy. Nanomedicine. 2013;8(1):17-28. DOI: 10.2217/nnm.12.82

[33] Turkevich J, Stevenson PC, Hillier J. A study of the nucleation and growth processes in the synthesis of colloidal gold. Discuss Faraday Soc. 1951;11:55-75. DOI: 10.1039/ DF9511100055

[34] Corbierre MK, Cameron NS, Lennox RB. Polymer-stabilized gold nanoparticles with high grafting densities. Langmuir. 2004;20(7):2867-73. DOI: 10.1021/la0355702

[35] Boca SC, Astilean S. Detoxification of gold nanorods by conjugation with thiolated poly (ethylene glycol) and their assessment as SERS-active carriers of Raman tags. Nanotechnology. 2010;21(23):235601. DOI: 10.1088/0957-4484/21/23/235601

[36] Nerambourg N, Werts MH, Charlot M, Blanchard-Desce M. Quenching of molecular fluorescence on the surface of monolayer-protected gold nanoparticles investigated using place exchange equilibria. Langmuir. 2007;23(10):5563-70. DOI: 10.1021/ la070005a

[37] Zhang X, Servos MR, Liu J. Instantaneous and quantitative functionalization of gold nanoparticles with thiolated DNA using a $\mathrm{pH}$-assisted and surfactant-free route. J Am Chem Soc. 2012;134(17):7266-9. DOI: 10.1021/ja3014055

[38] Behnke T, Mathejczyk JE, Brehm R, Würth C, Gomes FR, Dullin C, Napp J, Alves F, Resch-Genger $\mathrm{U}$. Target-specific nanoparticles containing a broad band emissive NIR dye for the sensitive detection and characterization of tumor development. Biomaterials. 2013;34(1):160-70. DOI: 10.1016/j.biomaterials.2012.09.028

[39] Joshi PP, Yoon SJ, Hardin WG, Emelianov S, Sokolov KV. Conjugation of antibodies to gold nanorods through Fc portion: synthesis and molecular specific imaging. Bioconj Chem. 2013;24(6):878-88. DOI: 10.1021/bc3004815

[40] Warner MG, Reed SM, Hutchison JE. Small, water-soluble, ligand-stabilized gold nanoparticles synthesized by interfacial ligand exchange reactions. Chem Mater. 2000;12(11):3316-20. DOI: 10.1021/cm0003875

[41] Connor EE, Mwamuka J, Gole A, Murphy CJ, Wyatt MD. Gold nanoparticles are taken up by human cells but do not cause acute cytotoxicity. Small. 2005;1(3):325-7. DOI: 10.1002/smll.200400093

[42] Ding H, Yong KT, Roy I, Pudavar HE, Law WC, Bergey EJ, Prasad PN. Gold nanorods coated with multilayer polyelectrolyte as contrast agents for multimodal imaging. J Phys Chem C. 2007;111(34):12552-7. DOI: 10.1021/jp0733419

[43] Chang JY, Wu H, Chen H, Ling YC, Tan W. Oriented assembly of Au nanorods using biorecognition system. Chem Comm. 2005;(8):1092-4. DOI: 10.1039/B414059A 
[44] Huang HC, Barua S, Kay DB, Rege K. Simultaneous enhancement of photothermal stability and gene delivery efficacy of gold nanorods using polyelectrolytes. ACS Nano. 2009;3(10):2941-52. DOI: 10.1021/nn900947a

[45] Kirui DK, Krishnan S, Strickland AD, Batt CA. PAA-derived gold nanorods for cellular targeting and photothermal therapy. Macromol Biosci. 2011;11(6):779-88. DOI: 10.1002/ mabi.201100050

[46] Gorelikov I, Matsuura N. Single-step coating of mesoporous silica on cetyltrimethyl ammonium bromide-capped nanoparticles. Nano Lett. 2008;8(1):369-73. DOI: 10.1021/ nl0727415

[47] Consani C, Auböck G, Van Mourik F, Chergui M. Ultrafast tryptophan-to-heme electron transfer in myoglobins revealed by UV 2D spectroscopy. Science. 2013;339(6127):1586-9. DOI: 10.1126/science.1230758

[48] Du C, Deng D, Shan L, Wan S, Cao J, Tian J, Achilefu S, Gu Y. A pH-sensitive doxorubicin prodrug based on folate-conjugated BSA for tumor-targeted drug delivery. Biomaterials. 2013;34(12):3087-97. DOI: 10.1016/j.biomaterials.2013.01.041

[49] Burstein E, Vedenkina N, Ivkova M. Fluorescence and the location of tryptophan residues in protein molecules. Photochem Photobiol. 1973;18(4):263-79. DOI: 10.1111/j.1751-1097.1973.tb06422.x

[50] Bhushan B, Dubey P, Kumar SU, Sachdev A, Matai I, Gopinath P. Bionanotherapeutics: niclosamide encapsulated albumin nanoparticles as a novel drug delivery system for cancer therapy. RSC Adv. 2015;5(16):12078-86. DOI: 10.1039/C4RA15233F

[51] Peters T. Serum albumin. Adv Prot Chem. 1985;37:161-245.

[52] Naik P, Chimatadar S, Nandibewoor S. Interaction between a potent corticosteroid drug-dexamethasone with bovine serum albumin and human serum albumin: a fluorescence quenching and fourier transformation infrared spectroscopy study. J Photochem Photobiol B Biol. 2010;100(3):147-59. DOI: 10.1016/j.jphotobiol.2010.05.014

[53] Kandagal P, Ashoka S, Seetharamappa J, Shaikh S, Jadegoud Y, Ijare O. Study of the interaction of an anticancer drug with human and bovine serum albumin: spectroscopic approach. J Pharm Biomed Anal. 2006;41(2):393-9. DOI: 10.1016/j.jpba.2005.11.037

[54] Tian J, Liu J, Tian, X, Hu Z, Chen X. Study of the interaction of kaempferol with bovine serum albumin. J Mol Struct. 2004;691(1):197-202. DOI: 10.1016/j.molstruc.2003.12.019

[55] Zhou J, Wu X, Yang C, Gu X, Zhou L, Song K, Feng Y, Shen J. Spectroscopic studies on the interaction of hypocrellin A with myoglobin. J Spectros. 2007;21(4):235-43. DOI: $10.1155 / 2007 / 503537$

[56] Giugliarelli A, Tarpani L, Latterini L, Morresi A, Paolantoni M, Sassi P. Spectroscopic and microscopic studies of aggregation and fibrillation of lysozyme in water/ethanol solutions. J Phys Chem B. 2015;119(41):13009-17. DOI: 10.1021/acs.jpcb.5b07487 
[57] Grinvald A, Steinberg I. The fluorescence decay of tryptophan residues in native and denatured proteins. BBA-Protein Struct. 1976;427(2):663-78. DOI: 10.1016/0005-2795(76)90210-5

[58] Lakowicz JR. (2013) Principles of fluorescence spectroscopy. Berlin: Springer. DOI: 10.1007/978-0-387-46312-4

[59] Vivian JT, Callis PR. Mechanisms of tryptophan fluorescence shifts in proteins. Biophys J. 2001;80(5):2093-109. DOI: 10.1016/S0006-3495(01)76183-8

[60] Latterini L, Tarpani L. (2012) AFM measurements to investigate particulates and their interactions with biological macromolecules. In Atomic force microscopy investigations into biology - from cell to protein. Rijeka: Intech. DOI: 10.5772/2092 

Chapter 2

\title{
Micro- and Nanocarriers for Immobilization of Enzymes
}

\author{
Maja Leitgeb, Željko Knez and Katja Vasić \\ Additional information is available at the end of the chapter \\ http://dx.doi.org/10.5772/63129
}

\begin{abstract}
Two types of micro- and nanocarriers for immobilization of enzymes for biotechnological and biomedical applications are described: magnetic nanoparticles and crosslinked enzyme aggregates (CLEAs). Nanosized structures with their large surface and smaller size volume ratio, which is dependent on their strong magnetic dipole, give key features that make magnetic nanoparticles useful in many biotechnological and biomedical applications. They are therefore used as carriers to which different active substances can bind. The preparation of the magnetic nanoparticles, possible surface coating methods, and functionalization with different materials are described. Enzyme immobilization methods, such as adsorption, affinity binding, chelation, or metal binding or covalent binding, enable the preparation of efficient and stable enzyme bound to magnetic nanoparticles. Such a product may be used among bioreactor applications for targeted drug delivery in biosensors or bioimaging and magnetic resonance imaging. Preparation of CLEAs, the microsized enzyme structures without a carrier, is described as well. Their main advantage is very simple preparation, where two steps, precipitation of the enzyme and cross-linking, are joined. A broad spectrum of enzymes for CLEA preparation has been used and many biotechnological reactions are catalyzed. The improvement in CLEA preparation to enhance their stability and operability is also shown.
\end{abstract}

Keywords: Biotechnology, Biomedicine, Enzymes, immobilization, carriers, nanotechnology.

\section{Introduction}

Thirty years ago, the first chemical immobilization of proteins and enzymes was performed. Immobilized proteins are currently used for processing of several products in different areas: from food industry to environment and medicine. Their use is also in many bioanalytical and 
biomedical applications, which include the use of immobilized antibodies or antigens in bioaffinity chromatography, of immobilized receptors or ligands, and of immobilized cells in biosensors.

Because free enzymes show poor stability towards heat and $\mathrm{pH}$, they offer limited possibilities of its reusability and recovery. To improve those main characteristics, new approaches are constantly producing new ways of improvement in enzyme stability and reusability with growing tools, such as immobilization techniques. New immobilization techniques offer new improved materials, which provide high-performance biocatalysts and favorable environments in which immobilized enzymes can work. These environments include much wider $\mathrm{pH}$ and temperature range and give more activity and stability than free enzymes [1-5]. Numerous applications of magnetic micro- and nanoparticles in biotechnology and biomedicine have been expanding and growing over the last decade due to their tremendous potential in targeting and delivery systems and due to their advantages in biosubstance binding. The development and synthesis of such materials have contributed immensely to several research disciplines, such as pharmaceutical, biomedical, and biotechnological to physics and electronics, to advanced materials, and to chemical science in general in a form of imaging agents, sensors, drug delivery targets/vehicles, and diagnostic tools. A great contribution was made to improve engineering skills in surgical diagnosis, therapy, and treatment [6-9]. The advantages and applicability of nanoparticles has opened up a large scope of studies. The main advantages of nanoparticles, compared to larger-sized particles, are their high surface-tovolume ratio and hence higher surface energy and excellent magnetic properties and, of course, their small size (approximately 1-100 nm). Their inorganic magnetic core is usually surrounded by layers of functional coatings. Their large surface and smaller size volume ratio, which is dependent on their strong magnetic dipole, gives key features that make them useful in many biotechnological and biomedical applications. Magnetic nanoparticles are therefore used as carriers to which different active substances can bind. They also have high coercivity and high magnetic susceptibility and are often bound together in aggregates, which give them many new possibilities in properties and functionalization. High surface area of magnetic nanoparticles allows the improvement of its bioapplicability and bioavailability, which allows larger dosage of bulk drugs and its pharmacokinetic properties and increase of vascular circulation. In nanoparticle applications, their properties are mainly used to develop drugs for targeted delivery systems and for diagnostic purposes, as they are a good contrast device for magnetic resonance imaging (MRI). Magnetic nanoparticles are able to concentrate in a specific tissue or anatomical location. The results of clinical studies may help us determine the key substances for immobilization on magnetic nanoparticles with the aim to gain better control of four healing processes: migration and proliferation of fibroblasts, deposition of extracellular matrix, angiogenesis, and transformation (scarring). At the same time, the optical properties of magnetic nanoparticles are used as alternatives to organic dyes for imaging purposes and also provide enhanced target specificity, which makes them an attractive drug delivery vehicle with an advantage and possibility of monitored drug release. Magnetic nanoparticles coated with different materials give more advantages in biological applications and provides an improvement in properties. Those properties can provide less cytotoxicity, improve biocompatibility, and provide better conjugation with other biologically active substances. Because 
magnetic nanoparticles are generally toxic to a living system, when they penetrate through membranes and interfere with basal metabolic reactions within the cell, a form of a coating on top of the core particle can make the magnetic nanoparticle less toxic and more biocompatible. The conjugation of biologically active substances and other suitable proteins onto its surface can improve many bioapplications. Magnetic nanoparticles modified with different surface substances are able to improve the surface chemistry of the nanoparticles to increase its affinity to bind different biologically active substances, proteins, DNA complexes, drugs, receptors, etc. All these modifications have led to improvements in the synthesis and preparations of magnetic nanoparticles that have to be in sync with a certain biological system. Therefore, biocompatibility and cytocompatibility are the most important aspects to increase the therapeutic value in using magnetic nanoparticles as drug delivery vehicles and furthermore in controlling drug release [10-14].

Another interesting area are micro- and nanosized enzyme cross-linked aggregates without additional carrier. Due to their extremely high activity, they are welcome in biotechnology and in biomedicine applications as biosensors.

\subsection{Immobilized enzymes in biotechnology}

Over the past decades, immobilized enzymes have affected numerous technologies in industrial processes. The ability of the enzymes to catalyze the reaction has made them indispensable to science for many years. Because one of the most important roles of enzymes as natural biocatalysts is their ability to enhance the rate of all chemical reactions, they increase the rates of chemical reactions without their altering or being consumed by the reaction and without changing the equilibrium between reactants and products. The most valuable properties of immobilized enzymes are their ability of easy reusability, lower degradation, higher control over the reaction rate, and ability to prevent contamination of the substrate with enzyme or protein. Also, the immobilization of the enzymes has been shown to improve the stability of the enzymes and to lengthen their half-life. It offers the enzymes to work in a larger range of environments, which improves enzyme stability against temperature, solvents, $\mathrm{pH}$, contaminants, and impurities. The immobilization of the enzymes increases the productivity of biocatalysts and enhances their features, which makes them more attractive and highly applicable in all kinds of evolving biotechnologies.

The enzyme, the matrix, and the mode of attachment are the main components of an enzyme immobilization, where the main advantages are the improvement of enzyme stability, increase of volume-specific enzyme loading, and simplification of biocatalyst recycling $[1,3]$.

Immobilized enzymes have been employed in reactions in either a batch or a continuous flow reactor. Batch reactors are applicable for smaller productions. They are suitable for expensive enzymes, as they can be used in small amounts and are not wasteful. Continuous reactors are suitable for increasing the time of reactions as well as the efficiency of a reaction using biocatalysts.

With recent advances and improvements in biotechnology, immobilized enzymes have been used in a wide range of applications. The advances include the improvements of immobiliza- 
tion techniques, new methods for the production or breakdown of compounds using biocatalytic reactions, methods of targeted drug delivery and tumor identification, and advances in using immobilized enzymes in biosensors. The most frequent enzyme immobilization is used for the synthesis of chemicals, where the industrial applications are straightforward. Through biocatalyzed reactions, they provide a reusable method for the production of chemicals and can break down harmful or undesired compounds. To develop stable and recoverable enzymes for multipurpose biotechnological applications and to increase their utilization as industrial biocatalysts, biocatalysts must be obtained from enzymes isolated from microbes, which naturally exist in extreme environments. Another way of obtaining stable enzymes is genetic engineering, which involves isolation. The most common and best way of obtaining stable enzymes is achieved by immobilization, protein engineering, and chemical modifications. Protein engineering has been able to produce enzymes that differ from their native forms in only several or one amino acids. This approach is offering many possibilities of stabilizing enzymes by changes in amino acid sequences [15-25].

\subsection{Immobilized enzymes in biomedicine}

Currently, the routine use of immobilized proteins or enzymes in medical field is in the diagnosis and treatment of various diseases. Immobilized antibodies, receptors, and enzymes are used as biosensors for the detection of different bioactive compounds in diagnosis. Encapsulated enzymes are used in different types of bioreactors for the separation of metabolites from body fluids or for the improvement of metabolic deficiency in the human body. Artificial cells and the development of controlled drug delivery systems for dosing of proteins or enzymes is also an application of immobilized enzymes in biomedicine. One of the applications of immobilized enzymes in medicine are also enzyme-based electrodes. High specificity and reactivity of an enzyme towards its substrate is used in biosensors, which have high reliability, sensitivity, accuracy, and ease of handling and are relatively low cost compared to conventional analytical methods. These biosensors are currently used in the clinical diagnosis of glucose [26-30], exalate [31,32], urea [33,34], glutamate [35,36], theophylline [37], creatine and creatinine [38,39], cholesterol $[40,41]$, and bilirubin $[42,43]$.

The application of immobilized enzymes in bioreactions is in the convection of inborn errors of metabolism, cancer treatment, and separation of waste products from body fluids. Enzyme or enzymes could be immobilized on solid support, encapsulated in sol-gel support, or alternatively cross-linked without a support to form the bioreactor.

Some applications of bioreactors used in human medicine are in areas of

a. Degradation of organophosphate in organophosphate poisoning by the use of phosphotriesterases [44,45],

b. Conversion of alcohol to acetate in alcohol poisoning by the use of alcohol dehydrogenase $(\mathrm{ADH})$ and acetaldehyde dehydrogenase [46,47],

c. DNA damage repair in skin aging and cancer by DNA repair enzyme (liposome) $[48,49]$, 
d. Hydrolysis of phospholipids in hypercholesterolemia by the use of phospholipase $\mathrm{A}_{2}$ (liposome) [50], and

e. Conversion of ifosfamide or oxazaphosphorine to tumoridical metabolites in tumors by the use of cytochrome $P 450$ (cells encapsulated in cellulose sulfate formation) $[9,51,52]$.

\section{Magnetic nanoparticles as nanocarriers}

Recent developments in life sciences in the last decade have produced numerous applications of magnetic nanoparticles in different fields of science and technology (biomedicine, biotechnology, biochemistry, etc.) and offer exciting new possibilities for applying new methods in an ever-growing and developing field of nanomedicine.

Superparamagnetic iron oxide (magnetite and maghemite) nanoparticles are the most used particles in several research disciplines due to their advantages in biosubstance binding and their tremendous potential in targeting and delivery systems. The main advantages of nanoparticles, compared to larger-sized particles, are their high surface-to-volume ratio and hence higher surface energy and excellent magnetic properties. Magnetic nanoparticles have been widely used in enzyme immobilization because of their unique properties, which among others include superparamagnetism and easy separation [29-31]. Together with the bound enzymes, they are interesting for the use in bioreactors. Among other advantages of magnetic nanoparticles, such as biocompatibility, small size, low toxicity, and, especially, superparamagnetism, a significant reduction of the biocatalyst amount used in a reaction was achieved at nicotinamide adenine dinucleotide hydrate (NADH) oxidation in a microreactor using $\mathrm{ADH}$-loaded magnetic nanoparticles. In a reactor system with an oscillating magnetic field and an actively moving ADH-loaded magnetic nanoparticles, 100\% NADH conversion was achieved for residence time of just $2 \mathrm{~min}$ [53].

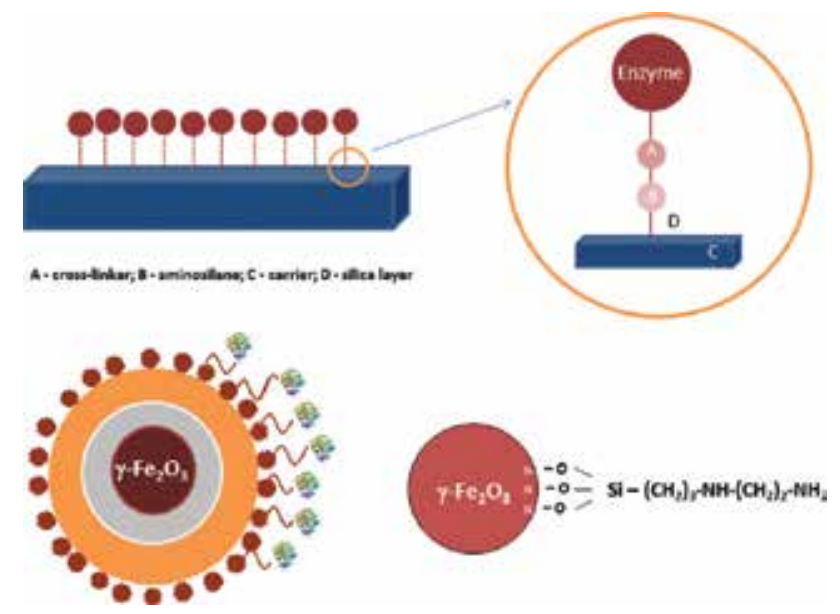

Figure 1. Magnetic nanoparticle surrounded by layers of functional coatings. 
Magnetic nanoparticles are typically crystals of inorganic elements for which the largest characteristic dimension is approximately 1 to $100 \mathrm{~nm}$. Their inorganic magnetic core is usually surrounded by layers of functional coatings (Figure 1).

Frequently used magnetic nanoparticles are also cobalt and nickel magnetic nanoparticles. Cobalt is a ferromagnetic element commonly used as an alloying element in permanent magnets. Nickel magnetic nanoparticles are of great interest due to their superparamagnetic and optical properties. There have been many protocols for the synthesis of cobalt or nickel magnetic nanoparticles, which include thermal decomposition, reverse micelles, chemical reduction, and polyol reduction, and for cobalt magnetic nanoparticles also sonochemical synthesis [54-62]. There are also many alloy-bimetallic magnetic nanoparticles that include various systems, such as iron-cobalt, nickel-cobalt, iron-platinum, and cobalt-platinum.

However, because of their low toxicity and biocompatibility, the most used magnetic nanoparticles are iron oxide nanoparticles [10,13,63,64]. Iron, being a ferromagnetic element, has the highest saturation magnetization at room temperature; therefore, iron magnetic nanoparticles exhibit superparamagnetic behavior in the size range below $20 \mathrm{~nm}$. In general, naked iron oxide magnetic nanoparticles are often highly reactive and can easily undergo degradation and oxidation, which leads to poor stability in strong acidic solutions and results in an iron oxide shell. Also, leaching can occur, which limits the reusability and lifetime of such materials [12,65-68]. The aggregation of the particles is also one problem, which is a result of large surface-to-volume ratio that presents another limitation. This limitation results in low surface energy that can occur due to strong magnetic attractions between particles and would result in the loss of activity or certain decrease of it.

To prevent and overcome such limitations, many various approaches can be used to modify the surface of magnetic nanoparticles. These approaches and techniques can improve and optimize their biocompatibility and biodegradability and, more importantly, can transfer a hydrophobic system into a hydrophilic one. To prevent oxidation and avoid instability in ambient conditions, iron magnetic nanoparticles must be protected by applying suitable coatings with materials that are chemically stable and biocompatible, such as gold ( $\mathrm{Au})$. $\mathrm{Au}$ shell makes a versatile surface for biofunctionalization due to its strong affinity towards thiols that are often present in biological molecules. Many versatile materials have been used in the application of these improvements. Surfactants, such as oleic acid, citric acid, lauric acid, alkylsulfonic acids, and alkylphosphonic acids, are commonly used. Also used as coatings are different polymers, such as polyethylene glycol (PEG), polyvinylpyrroline (PVP), and polyvinyl alcohol (PVA). More importantly, also natural materials, such as chitin, dextran, alginate, albumin, gelatin, starch, liposomes, and ethyl cellulose, are widely used and applied as coatings onto iron oxide magnetic nanoparticles, which are bound chemically or by multiple hydrogen bonds and therefore develop hydrophilic system [69-75]. Coatings on iron magnetic nanoparticles do not only influence the colloidal stability but also the biological functionality of nanoparticles. Good colloidal stability and the prevention of particle aggregation in a $\mathrm{pH}$ area of approximately 7.4 are the most important requirements for biomedical applications. Molecules on the surface of nanoparticles can interact in different ways. 
"Encapsulation" of nanoparticles in polymers can apply through polymerization in a suspension of nanoparticles or magnetic nanoparticle synthesis occurs in a polymeric media or polymer molecules are grafted at the surface of nanoparticles [76].

"Coating through hydrophobic interactions" is a strategy also named bilayer functionalization. Magnetic nanoparticles that are synthesized by thermal decomposition are capped by hydrophobic surfactants that are coated with amphiphilic molecules [77].

"Interactions using coupling agents or covalently binding" present an alternative to physisorption, as physisorbed molecules are easily desorbed when nanoparticles are introduced to physiological media and nanoparticles coated with adsorbed molecules tend to aggregate in phosphate-buffered saline (PBS), which is caused by the displacement of carboxylic acid groups from the nanoparticle surface by phosphate salts [78-80].

To improve coating stability, other high-affinity surface-capping agents are being explored (silane group, carboxylate, carboxylate and $\mathrm{OH}$ groups, catechol, hydroxamic acid, phosphonate groups, and inorganic coatings, which include silica, $\mathrm{Au}$, and other metals and metal oxides). A silica shell is mostly used for the modification and protection of magnetic core, where it can prevent unwanted interactions, such as acidic corrosion. Silica coating has many advantages and can provide better stability under aqueous conditions and better biocompatibility $[72,81,82]$.

Surface silanization is a commonly used method to introduce functional groups onto magnetic nanoparticles. The silanization process can widely improve and help forward to low cytotoxicity and high stability under acidic conditions and can ease the surface chemical modification. Reactions of silanization can be carried out in aqueous media or organic solvents at moderate temperatures. In the reaction mechanism, silane-based molecules such as 3-aminopropyl trimethoxysilane (APTMS) are covalently bound to the surface of nanoparticles to where other bioactive molecules can be further bound. The main advantage of a silane agent is its biocompatibility and a high density of surface functional end groups (alcohol, amine, thiol) to which other biocompatible components can bind and be functionalized $[76,80,83]$.

Another agent used for the modification of nanoparticle surface is carboxylic acid group, which can be employed with oleic acid used as a surfactant. Carboxylic groups are reportedly more active than hydroxyl groups. However, when the hydroxyl group is ortho-positioned regarding the carboxyl group, both groups will coordinate to the iron atom, forming a chelating structure. Hydroxamic acid derivates are mainly used in ligand exchange process to coat nanoparticles in situ with fatty acids during synthesis. Using bifunctional phosphonic acid groups with polar end groups $\left(-\mathrm{COOH},-\mathrm{NH}_{2},-\mathrm{OH}\right)$ provides functionality on the surface and a hydrophilic and stable system.

The size of the magnetic carriers can be engineered by their preparation technique. Chitosancoated magnetic nanoparticles were prepared using three different methods: microemulsion process, suspension cross-linking technique, and covalent binding method [84]. The size of the particles varied with the preparation method and was increased by the increase in the concentration of chitosan and decreased by the increase of the cross-linker concentration. Particles coated with chitosan had a spherical shape and a size range of 40 to $350 \mu \mathrm{m}$ using the 
microemulsion process, 10 to $50 \mu \mathrm{m}$ using the suspension cross-linking technique, and 50 to $100 \mathrm{~nm}$ using the covalent binding method. Magnetic nanoparticles coated with chitosan with the covalent binding method were the most suitable for practical applications due to their sufficiently high values of amino groups $(2.48 \mathrm{mmol} / \mathrm{g})$, which is important for protein immobilization [85].

Immobilization methods developed for enzyme/protein binding onto iron magnetic nanoparticles are mainly divided into physical immobilization, covalent conjugation, and biologically mediated specific interactions.

\subsection{Adsorption}

\subsubsection{Physical adsorption}

Physical immobilization, including physical adsorption, is considered as the simplest method for protein or enzyme immobilization. This method is based on the adsorption of enzyme or protein on the surface of water-insoluble carriers, which include porous carbon, ion-exchange matrices, or polymeric aromatic resins (Figure 2). Furthermore, the method can easily be carried out without any additional coupling agents, surface treatment, or enzyme/protein modification. Also, it makes no conformation changes to the enzyme and is simple and cheap. It can simply be performed by dipping the material into a certain solution containing our target bioactive substances [86-88]. In recent years, many physically immobilized enzymes based on iron magnetic nanoparticles were developed. For example, hybrid magnetic $\mathrm{Fe}_{3} \mathrm{O}_{4}-\mathrm{SiO}_{2}$ poly(ethylene oxide)-maltose nanoparticles were used as affinity adsorption carriers for the direct separation of maltose binding protein-fused heparinase I. The results demonstrated that the mentioned nanoparticles were suitable carriers for affinity purification and immobilization in a single step, showing high adsorption capacity [89]. Also, glucose oxidase was immobilized by employing tannic acid-modified $\mathrm{CoFe}_{2} \mathrm{O}_{4}$ magnetic nanoparticles. Using strong protein and tannic acid binding, glucose oxidase immobilization was carried out via physical adsorption, where it showed excellent reusability and where immobilized glucose oxidase maintained at $60 \%$ of its initial activity [90]. Feruloyl esterases (FAE) were also successfully immobilized on magnetic iron nanoparticles via physical adsorption. Optimal immobilization conditions were significantly influenced by $\mathrm{pH}$, where the maximum activity of immobilized FAEs were observed [91]. Because physical adsorption is a simple and mild method, it involves weak interactions, where enzymes are attached to the matrix through hydrogen bonds [92], van der Waals forces [93,94], and hydrophobic interactions [95]. Thermomyces lanuginosa lipase (TLL) was immobilized via physical adsorption onto the polyethyleneimine-coated iron magnetic nanoparticles that were prepared for the chelation of three metal ions. The immobilized lipase obtained more than $60 \%$ of initial activity after 10 cycles and retained about $80 \%$ of initial activity after 14 days of storage [96]. 


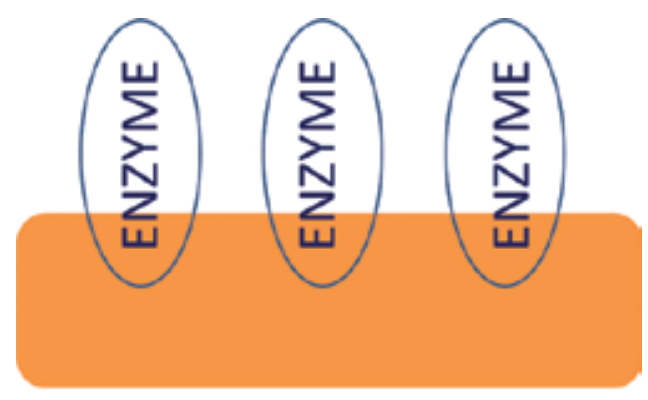

\section{Adsorption}

Figure 2. Adsorption of the enzyme on the surface of a carrier.

Forces involved in noncovalent immobilization result in a process that can be reversed by changing the environmental conditions, such as $\mathrm{pH}$, temperature, ionic strength, polarity of the solvent, and concentration of bioactive molecules. Immobilization by adsorption usually preserves the catalytic activity of the enzyme, which makes them economically attractive. However, because these interactions in physical adsorption are relatively weak, they often result in breaking away from the support or in enzyme leakage from matrix, which can lead to the loss of activity and denaturation of the protein or enzyme.

\subsubsection{Affinity binding}

Affinity binding has well been applied to enzyme immobilization, where the most important advantage is the selectivity of the interactions. The method often requires covalent binding of an expensive ligand to the matrix. For example, an oriented immobilization of chloroperoxidase on iron magnetic nanoparticles was achieved by layer-by-layer controlled assembly through specific interactions of avidin-biotin affinity binding [97].

\subsubsection{Chelation or metal binding}

Another method of reversible immobilization is also presented in chelation or metal binding, where metal salts or hydroxides on the surface of organic carriers are bound by coordination with nucleophilic groups on the matrix. The metal salt or hydroxide is precipitated on the support (chitin, alginate, silica-based carriers) by heating or neutralization. Some of the positions on the metal remain free, as it is impossible for the matrix to occupy all positions on the metal. This occurs because of the steric factors. The method is simple and can obtain relatively high remaining specific activities of the enzyme (30-80\%). For example, $\beta$-Dglucosidase was immobilized on iron magnetic nanoparticles coupled with agarose via metal ion affinity between alkaline amino groups located on the surface of the $\beta$-D-glucosidase and the $\mathrm{Co}^{2+}$ chelated on the carrier [98]. Another example shows that metal chelate ions $\left(\mathrm{Co}^{2+}\right.$, $\mathrm{Cu}^{2+}, \mathrm{Pd}^{2+}$ ) increase the capacity of enzyme immobilization, where polyethyleneimine-coated iron magnetic nanoparticles were prepared via chelation [96]. 


\subsection{Covalent binding}

Covalent immobilization is the most widely used immobilization method and an attractive one, as it can be regulated with specific functional groups to bind enzymes or proteins. The main advantage of covalent immobilization is that the bonds formed between the enzyme and the matrix are of stable nature, so the enzyme cannot be released into the solution and the enzyme leakage is absent or limited. There are several immobilization protocols that are being researched using covalent binding and applying it in enzyme immobilization [1,99-101]. For improving enzyme activity, a coupling reaction can be applied using coupling agents, such as glutaraldehyde (GA) [72,99,102-105] or 1-ethyl-3-(3-dimethylaminopropyl) carbodiimide hydrochloride (EDC) [106,107]. GA and EDC are because of their functional group (aldehyde group) mostly covalently cross-linked to magnetic nanoparticles and enzymes/proteins due to their interaction with both functional groups (Figure 3). However, in some cases, the presence of a coupling agent can also cause conformational changes in proteins, which can result in a decrease of enzyme activity.

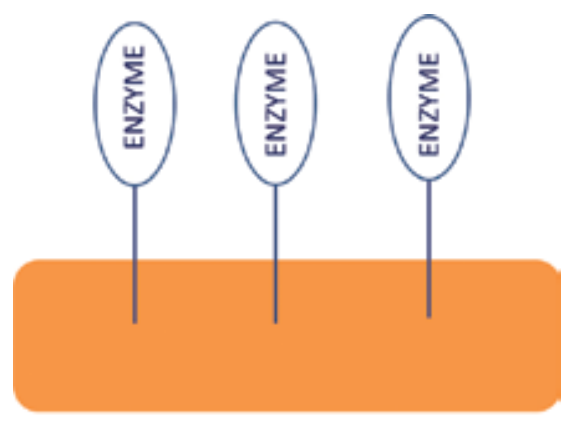

\section{Covalent binding}

Figure 3. Covalent immobilization of the enzyme.

There are many researches performed, where enzymes were covalently immobilized onto iron magnetic nanoparticles. For example, recombinant trehalose synthase was immobilized onto glutaraldehyde-activated silanized iron magnetic nanoparticles by covalent binding, where it retained $82 \%$ of its initial activity after successive 12 repeated cycles of reaction [108]. Another research reports the covalent coupling of lipase using EDC onto chitosan magnetic nanoparticles, where the immobilization of lipase was performed by the formation of an amide bond between the carboxy group of lipase and the primary amino group of the nanoparticle. Lipase immobilized onto the magnetic support demonstrated high catalytic activity and retained $78 \%$ of its initial activity [109]. Also, superparamagnetic nanoparticles were surface modified with a mixture of two polymers for lipase immobilization, where the lipase was covalently bound to the surface of nanoparticles via EDC/N-hydroxysulfosuccinimide sodium salt (sulfo-NHS) activation and retained $82 \%$ of its initial activity [73]. Another study reports recombinant acetyl xylan esterase (rAXE) being covalently immobilized with chitosan magnetic nanoparticles using glutaraldehyde and demonstrating better stability at thermal and $\mathrm{pH}$ ranges than soluble 
free rAXE. The immobilized rAXE was stable for about $90 \%$ of activity in aqueous phase and retained only $60 \%$ of its initial activity after 10 cycles of reuse [105]. Furthermore, serraptiopeptidase enzyme was immobilized by covalent binding through glutaraldehyde after aminofunctionalization of magnetic nanoparticles. The immobilized enzyme showed $67 \%$ of residual activity and good storage stability [110]. $\alpha$-Acetolactate decarboxylase ( $\alpha$-ALDC) was covalently immobilized to paramagnetic $\mathrm{Fe}_{3} \mathrm{O}_{4}-\mathrm{Si}^{-} \mathrm{NH}_{2}$ nanoparticles using 1'-carbonyldiimidazole (CDI). Such immobilized $\alpha$-ALDC retained almost $65.35 \%$ of its initial activity and $31.05 \%$ of its initial activity after five times of reusing [111]. Another study reports of cholesterol oxidase (COX) immobilization to magnetic fluorescent core-shell structured nanoparticles, where immobilization consisted of silanization, linker molecule deposition, and enzyme coupling of GA and 3-aminopropyltriethoxysilane (APTES). Therefore, immobilized COX maintained $80 \%$ of its initial activity and $71 \%$ of its initial activity after seven consecutive operations [103]. AiiA, which is a 28-kDa lactonase, was covalently immobilized onto magnetic nanoparticles, where immobilized recombinant AiiA ( $\mathrm{r}$-AiiA) was evaluated for its ability to hydrolyze and inhibit bacterial quorum sensing [112]. An interesting research was presented where pullulanase was immobilized onto magnetic chitosan activated nanoparticles prepared by in situ mineralization, showing that, although the immobilization by electrostatic adsorption can preserve higher activity of the enzyme (87\%), the poor stability of the immobilized pullulanase cannot be avoided. Covalent immobilization by cross-linking maintained $70 \%$ of initial activity but gave smaller activity losses and more improvement between enzyme and substrate affinity and gave more stability, as the covalent attachment formed between amino groups of the enzyme and support showed more catalytic activity than between the carboxy groups of the enzyme and support [113]. Another study describes COX covalently immobilized onto magnetic nanoparticles of maghemite further functionalized with silica and amino-silane molecules. Therefore, prepared nanoparticles immobilized with COX maintained up to $60 \%$ of initial activity [114], where another study shows how Aspergillus niger xylanase A (XylA) was covalently immobilized onto chitosan-coated magnetic nanoparticles by forming covalent bonds between the aldehyde group of glutaraldehyde and the amino group of the enzyme. The retained activity of immobilized XylA was $56.5 \%$ of its initial activity [115]. The immobilization of yeast ADH (YADH) from Saccharomyces cerevisiae onto chitosan-coated magnetic nanoparticles was performed by covalent binding, where different preparation conditions were taken into account, such as immobilization time, $\mathrm{pH}$, and enzyme concentration. Finally, the optimum reaction temperature was $30^{\circ} \mathrm{C}$ with $\mathrm{pH}$ of 7.4 and the retained activity of immobilized YADH was $65 \%$ of its original initial activity [71]. Another work presents $\alpha$ amylase being covalently immobilized using glutaraldehyde onto magnetic nanoparticles using gum acacia as the steric stabilizer. GA magnetic nanoparticles have been demonstrated to be capable of being reused for at least six cycles while retaining about $70 \%$ of the initial activity [116]. Another lipase immobilization was performed by both physical adsorption and covalent binding onto magnetic nanoparticles, of which the surface was grafted by block copolymer of 2-dimethylaminoethyl methacrylate (DMAEMA) and glycidyl methacrylate (GMA). The activity recovery of the immobilization lipase reaches $43.1 \%$ at the protein loading of $0.5 \mathrm{mg}$ (per $\mathrm{mg}$ of support) and retains $73 \%$ of its initial activity at $65^{\circ} \mathrm{C}$ for $8 \mathrm{~h}$ and its residual activity is reportedly more than $55 \%$ after six consecutive cycles [117]. 
On the contrary, a biosorbent was successfully prepared by the immobilization of Phanerochaete chrysosporium with iron oxide magnetic nanoparticles and calcium alginate, which is capable of removing $\mathrm{Pb}$ (II) ions from solutions, as $\mathrm{P}$. chrysosporium was reported for the biosorption of heavy metals, taking advantages of favorable heavy metal affinity. In this case, the immobilization assists native microbes in improving their biosorption capacity. The biosorption efficiency was reportedly highly time and $\mathrm{pH}$ dependent and affected by initial $\mathrm{Pb}$ (II) concentration [118].

\subsection{Targeted drug delivery using magnetic nanoparticles}

There are many advantages when talking about targeted drug delivery systems based on magnetic nanoparticles. Compared to other conventional drug carriers, magnetic nanoparticles have a better retention ability and are therefore promising simulative carriers that can transport a drug to the desired place in the body. Nanotechnology in biomedicine is mostly used for intravenous delivery, especially to cancer tissues. Another advantage of magnetic nanoparticles for targeted drug delivery is that nanoparticles as carriers can also be coated with different biodegradable organic polymers to enable interaction by binding with active molecules and to achieve a sufficient drug load. Thus, such system allows biodegradability, biocompatibility, nontoxicity, and prolonged circulation of a specific therapeutic or drug agent. Once the drug carrier is concentrated at our desired tissue location, the drug can be released either by enzymatic activity or by changes in physiological conditions, such as temperature, $\mathrm{pH}$ or osmolality, as can be seen in Figure 4 .
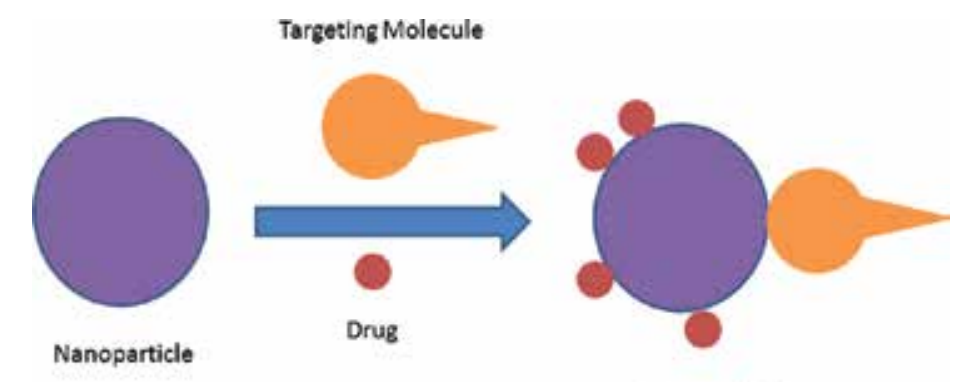

Targeted Delivery

Figure 4. Targeted drug delivery: targeting of a nanoparticle includes targeting ligands, such as antibodies or peptides that specifically bind to receptors expressed on target side.

An extensive research has been made regarding targeted drug delivery systems using magnetic nanoparticles. For example, magnetic molecularly imprinted polymer was synthesized using polydopamine, which was used for controlled 5-fluorouracil delivery in a spontaneous model of breast adenocarcinoma in BALB/c mice in the presence of an external magnetic field [119]. Another study investigates magnetic nanoparticles functionalized with thiol-terminated polyethylene glycol, loaded with anticancer drug doxorubicin (DOX) used as a biodegradable system for targeted delivery [120], whereas another research explores the same drug DOX delivered with $\beta$-cyclodextrin assembled magnetic nanoparticles, which were 
fabricated with layer-by-layer method to develop a versatile biocompatible platform for selective loading, targeted delivery, and $\mathrm{pH}$-responsive release of mentioned drug [8].

\subsection{Magnetic nanoparticles in biosensors}

Iron magnetic nanoparticles can also be coated with fluorescent materials or with silica, metal, or a polymer. Magnetic nanoparticles coated with such materials are used as bioanalytical sensors. In developing new biosensors, Au nanoparticles show good mechanical resistance and electrical properties as well as good biocompatibility. As all magnetic nanoparticles, they have large surface area for enzyme or protein immobilization. Biosensors contain layers that are bioselective, which can react with a target biomolecule that transforms the biologic interaction into physical signal (optical, chemical, electrical, thermal, etc.). The principle of biosensors is represented in Figure 5.

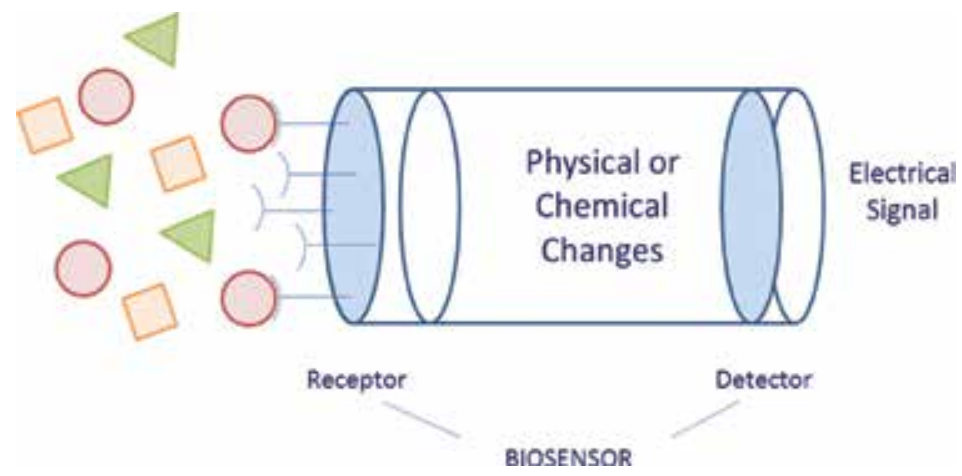

Figure 5. Principle of biosensors: transforming biological interaction into electrical signal.

A study proposes a biosensor using glassy carbon electrode modified with Au nanoparticles and tyrosinase, which is covalently immobilized in a dihexadecylphosphate film. Therefore, modified electrode is used to determine catechol concentrations by amperometry in natural water samples [121].

Biosensors are also applied to the determination of hepatitis B virus (HBV) DNA in urine and blood plasma samples. As described in a recent study, a magnetite and Au nanoparticlemodified paste electrode was prepared for the immobilization of thiol-modified HBV probe DNA. Therefore, developed biosensor could measure target HBV DNA virus concentration with low concentrations [122]. Later on, an ultrasensitive electrochemical DNA biosensor based on in situ labeling Au nanoparticles on the free terminal of hairpin DNA was developed, with good regeneration, stability, and hybridization selectivity [123]. Another paper describes Au nanoparticles being used to enhance electrochemical DNA biosensor, where the goal was to improve the sensitivity of biosensor by detecting small sequences in large amounts of double-stranded DNA [124]. Magnetic fluorescent core-shell nanoparticles immobilized with COX were prepared to be used as a sensing material with application in multiparameter fiberoptic biosensor based on enzyme catalysis and oxygen consumption [125]. 


\subsection{Bioimaging and MRI using magnetic nanoparticles}

MRI is a commonly used medical imaging technique that is a powerful and noninvasive tool used for the visualization of structures and functions in tissue. MRI is based on the detection of proton relaxation and an external magnetic field. Different enzymes and proteins immobilized on magnetic nanoparticles are largely applied in MRI imaging mostly because of their biocompatibility, superparamagnetism, low toxicity, colloidal stability at physiological conditions $\left(37^{\circ} \mathrm{C}, \mathrm{pH} 7.4\right.$, PBS buffer), and well-controlled surface charges. For example, magnetite nanoparticles were functionalized with mebrofenin, as it is known as a liver targeting function. Therefore, functionalized nanoparticles coated with silica were prepared as liver targeting contrast agents in MRI [126]. In another study, magnetic nanoparticles were used as drug carriers, aiming to form a magnetically controlled nanoplatform [127].

\section{Cross-linked enzyme aggregates (CLEAs) as microcarriers}

Many biotechnological approaches for production cover the synthesis of drugs. A lot of these enantiopure compounds, such as anticancer, antiviral, anti-infective, antipsychotic, antiarrhythmic, cholesterol-lowering agents, calcium channel blockers, ACE inhibitors, and many others, may be synthesized enzymatically.

Via immobilization of enzymes, stabilization occurs. Intermolecular interactions are prevented [100] and the enzyme structure becomes more rigid due to multipoint covalent attachment [1]. In multimeric enzymes, the dissociation of the subunits is prevented [128] and inhibition is reduced [100]. However, structural properties such as size, specific area, and porosity that dictate the resistance to mechanical stress are also important.

The most used cross-linking agent for enzyme immobilization is glutaraldehyde, as it is available in commercial quantity and is relatively cheap. Although its chemistry has been not fully understood, it is generally accepted that the inter- and intramolecular cross-linking reaction occurs between the free amino groups of lysine (Lys) residues on the enzyme or protein molecule and the carbonyl group of glutaraldehyde resulting in a Schiff base formation [129].

\subsection{Carrier-free immobilization}

The CLEA method is used for the dual purpose of combining both the purification and immobilization of enzyme in one step [130]. The first step is enzyme precipitation with the most suitable precipitating agent and the second step is enzyme cross-linking with a crosslinking reagent. An example of CLEA preparation is presented in Figure 1, where the scheme illustrates the preparation of CLEAs from horseradish peroxidase (HRP) [131]. Beside the precipitating agent and cross-linker, some other compounds are added to form the enzyme clusters. The reason for their use is described below (Figure 6). 


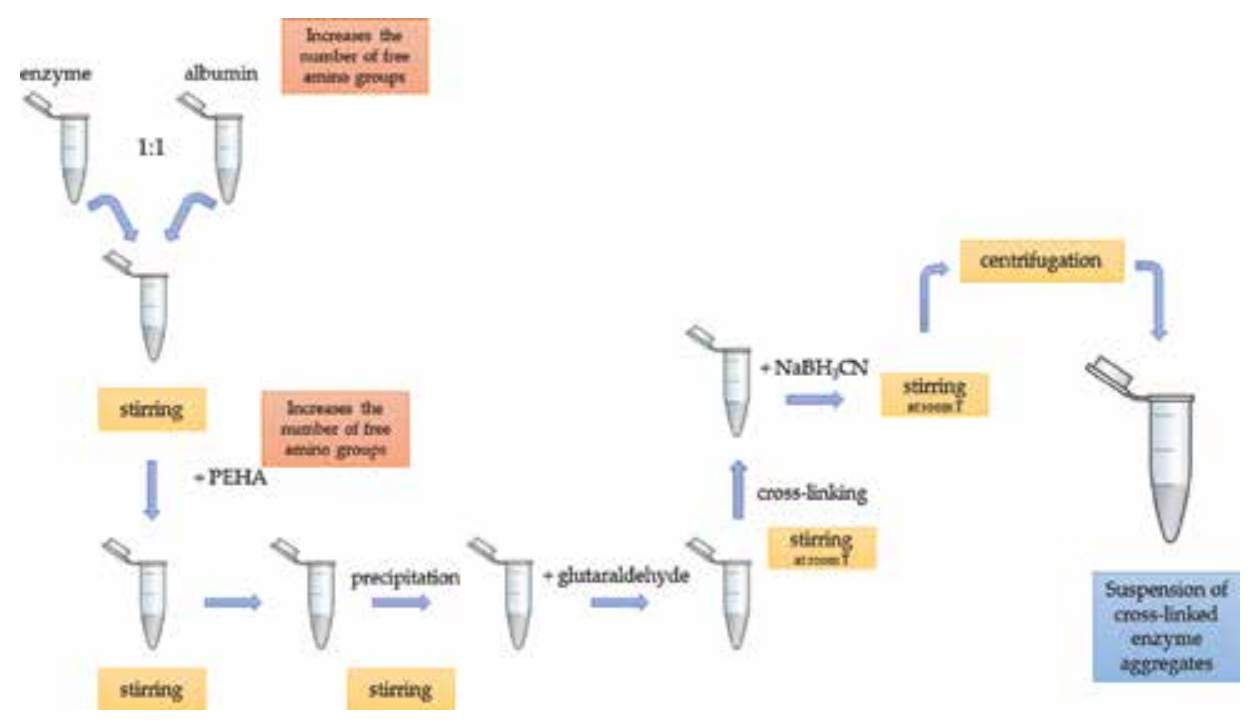

Figure 6. Preparation of CLEAs from HRP [131].

As mentioned before, the first step of CLEA preparation is the precipitation of the enzyme from the solution with the most suitable precipitating agent. Which precipitating agent is the most suitable one for a certain enzyme? This question may be answered after a simple experiment where the enzyme is precipitating with different precipitating agents and then resuspended again. The precipitating agent that gives the highest residual activity of the resuspended enzyme is the most suitable one. The most commonly used precipitating agents are acetone, methanol, ethanol, propanol, 2-propanol, and ammonium sulfate.

CLEAs are easily prepared from crude enzyme extracts, and the costs of (often expensive) carriers are circumvented. Many studies employed purified or partially purified enzyme samples for CLEA preparation [132-139]. On the contrary, it was successfully demonstrated that the lipase from Penicillium expansum (PEL)-CLEAs can be prepared directly from the fresh fermentation broth or its lyophilizate [140]. They generally exhibit improved storage and operational stability towards denaturation by heat, organic solvents, and autoproteolysis and are stable towards leaching in aqueous media. Furthermore, they have high catalyst productivities (kilogram product per kilogram biocatalyst) and are easy to recover and recycle. In CLEAs, immobilized enzymes are much more resistant to shear and extreme $\mathrm{pH}$ values than the soluble enzymes as well.

The particle size of CLEAs is usually between 5 and $50 \mu \mathrm{m}$, but it can be varied by changing the preparation parameters, such as precipitating agent or cross-linker concentration. Although with a smaller size of the clusters higher reaction rates are obtained, sometimes applications dictate the formation of bigger clusters. It is obvious that bigger particle sizes result in better filterability and smaller pressure drop in packed bed reactors. Table 1 represents particle size variations of CLEAs from cellulase (from 26 to $161 \mu \mathrm{m}$ ) in the dependence from the used precipitating agents. 


\begin{tabular}{ll}
\hline Precipitating agent & Particle size of CLEA clusters $(\mu \mathrm{m})$ \\
\hline Propanol & 26 \\
Ethanol & 43 \\
Acetone & 46 \\
Tetrahydrofurane & 67 \\
Methanol & 87 \\
2-Propanol & 161 \\
\hline
\end{tabular}

Table 1. Particle size deviation of CLEAs from cellulase.

However, the concentration of the cross-linking agent must be carefully optimized to prevent the enzyme activity loss. Namely, too big particle size of the enzyme preparation, which is formed with high glutaraldehyde concentration, may affect the mass transfer of the substrates to contact with the enzymes in the inner side. In this case, inner enzymes of the enzyme preparation lose the opportunity to form complexes with the substrates, which results in the apparent activity loss of the enzyme.

Scanning electron microscopy showed a uniform structure of the cross-linked $\alpha$-amylase aggregates. The diameter of CLEAs was about $250 \mathrm{~nm}$ with a small deviation (Figure 7). CLEAs

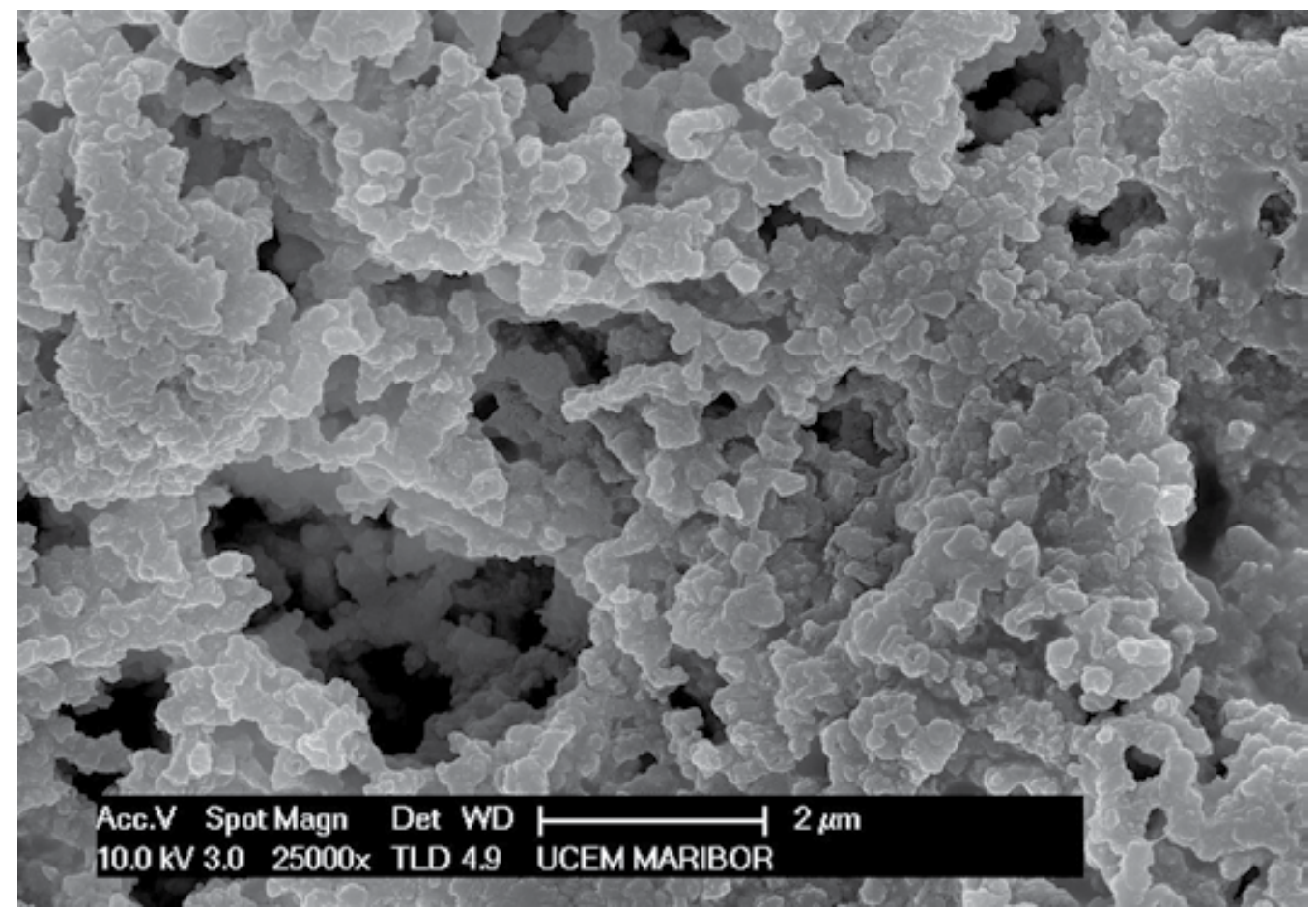

Figure 7. Scanning electron microscopy (SEM) image of CLEAs from $\alpha$-amylase. 
can form larger clusters that do have mass transfer limitations, especially in fast UV-based assays. One CLEA particle can contain up to 8 million enzyme molecules. The size of these clusters can be up to $100 \mu \mathrm{m}$, making them visible with the bare eyes. The number of CLEAs in a cluster is much less uniform than enzymes in an aggregate; it can vary from a few to many hundred thousands [141].

A broad spectrum of enzymes for CLEA preparation has been used. The majority of CLEAs that have been described involve enzymes, which belong to the class of hydrolases (proteases, amidases, lipases and esterases, glycosidases), oxidoreductases (oxidase, peroxidase), or lyases (nitril hydratase, hydroxinitril lyase), have been successfully immobilized as CLEAs [142]. The reason is probably because they are the enzymes that have the most industrial applications and are the simplest enzymes to work with.

With enzymes containing few or no accessible Lys residues, cross-linking may be insufficient and lead to CLEAs that are unstable towards leaching in aqueous media. One way to overcome this problem is to add polyamines, such as polyethyleneimine, which are then coimmobilized with the enzyme [143]. Problems can also be encountered in CLEA formation when the protein concentration in the enzyme preparation is low. In such cases, CLEA formation can be promoted by the addition of a second protein, such as bovine serum albumin (BSA), as a so-called proteic feeder [144]. A typical enzyme with low content of amine residues is aminoacylase [145]. Therefore, the immobilization of aminoacylase was improved by adding BSA to yield coaggregates [146]. Using BSA as a proteic feeder is also a solution in cases when the enzyme activity is vulnerable to high concentrations of glutaraldehyde required to obtain aggregates $[139,144]$. Namely, a high concentration of glutaraldehyde may reduce the activity of the obtained CLEAs, as free amino groups present in the proteic structure may react with this cross-linker [147] or it can bind amino acids associated with the active site.

These drawbacks can be prevented by the use of molecules with a large amount of Lys residues in the surface, such as BSA. With such a solution, the possibility of cluster formation, as in the case of excess of glutaraldehyde, with mass transfer limitations, may be prevented. Shah et al. prepared CLEAs of Pseudomonas cepacia lipase (PCL) with BSA addition. The recovered activity relative to that of free enzyme was $100 \%$ in contrast with the same preparation without BSA, which retained only $0.4 \%$ of activity [16]. More recently, the synthesis of CLEAs of lipase B from Candida antarctica (CALB) showed difficulties in the cross-linking step due to the low content of surface Lys on CALB [18]. The addition of BSA as a feeder allowed an effective crosslinking step and permitted to greatly stabilize CLEAs.

To enhance the activity of CLEA used enzymes, it is possible to use a modification of the CLEA synthesis protocol using proteic feeder. The enzyme could be added after cofeeder aggregates are formed and cross-linked. A slightly cross-linked layer of the enzyme is formed over the cofeeder cores, which might lead to enhanced enzyme-substrate contact (Figure 8). This approach was successfully demonstrated by Guauque Torres et al. [147] using TLL. The layer methodology led to high increase in retained activity compared to nonlayered TLL CLEAs. 


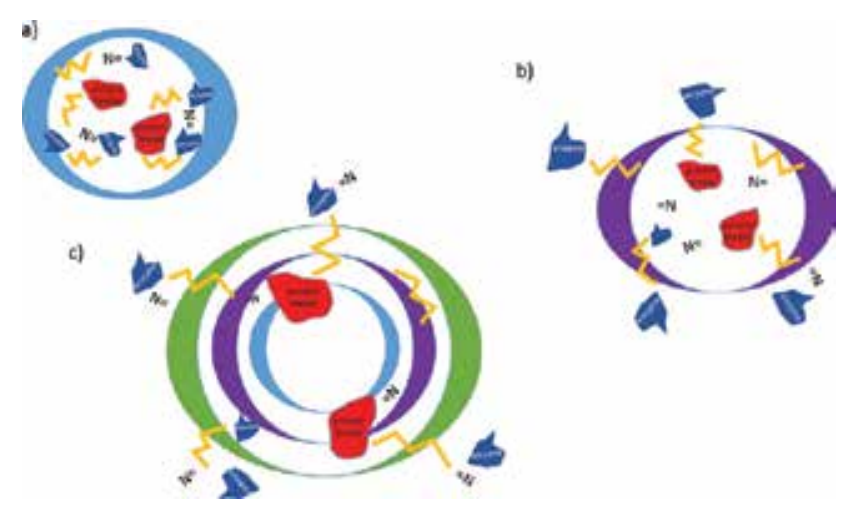

Figure 8. Nonlayered and layered structures of CLEAs: (a) nonlayered CLEAs, (b) one enzyme layer, and (c) three enzyme layers.

\subsection{CLEA formation using carriers}

There may appear some difficulties using CLEAs in a reaction system, as it is difficult to isolate and recover CLEAs from the reaction system only by centrifugation or filtration, especially when the substrate particles are in the same size range as CLEAs [148]. Therefore, some new reactor styles were designed [149-152]. Another way is to improve the operability and mechanical stability of CLEAs. For that reason, CLEAs of papain were prepared in commercial macroporous silica gel from simple adsorption, precipitation, and one-step cross-linking [148].

Another example was when CALB was adsorbed and cross-linked on a polypropylene carrier [153]. Such enzyme preparation maintained its activity when dispersed in ionic liquids, such as $[\mathrm{BMIm}]\left[\mathrm{NO}_{3}\right]$, which denatured the free enzyme. This is an important fact, as ionic liquids are, to an increasing degree, being accepted as reaction solvents, including biocatalytic transformations $[154,155]$. However, ionic liquids that contain strongly coordinating anions, such as [BMIm] nitrate or acetate, interact sufficiently strong with CALB to dissolve the enzyme but also cause its deactivation [156,157].

Laccase-based CLEAs were stabilized through the formation of a surrounding polymeric network made of chitosan and 3-aminopropyltriethoxysilane (EPES-lac). The average size of EPES-lac particles was about $100 \mu \mathrm{m}$ and with a spherical shape. The thermoresistance of the resulting enzyme polymer engineered structures of laccase CLEAs (EPES-CLEA) were more than 30 times higher than that of free laccase and CLEAs at $\mathrm{pH} 3$ and $40^{\circ} \mathrm{C}$. The formation of a polymer network around laccase-based CLEAs had a desirable effect on the biocatalysts formed in regard to chemical and physical characteristics, stability under harsh conditions, and kinetic parameters [158].

\subsection{A broad spectrum of biotechnological reactions may be catalyzed with CLEAs}

With CLEA of purified nitrilase from Escherichia coli harbouring gene of Pseudomonas putida, the hydrolysis of $(R S)$-mandelonitrile to $(R)$-mandelic acid, a versatile chiral building block, 
was performed by Kumar et al. [130]. (R)-mandelic acid is namely used as a chiral synthon for the production of antitumor and antiobesity agents [159].

The industrial production of enantiopure L-amino acids from N-acyl DL-amino acid can be performed using L-aminoacylase ( $\mathrm{N}$-acyl amino acid amidohydrolase or acylase I; E.C. 3.5.1.14), which has excellent chiral specificity and is one of the top 10 enzymes used in biotechnology [160]. This enzyme from Aspergillus sp. is useful for a broad spectrum of reactions, such as enantioselective hydrolysis of amino acid esters and amides [161], regioselective alcoholysis of carboxylic acid esters [162-164], acylation of primary and secondary alcohols [165-168], and acylation of amines [165-169]. Immobilization of L-aminoacylase from Aspergillus melleus using coaggregation with polyethyleneimine and subsequent cross-linking with glutaraldehyde resulted in stable aminoacylase-polyethyleneimine CLEAs (AP-CLEA) with significantly higher temperature and storage stability of the enzyme without affecting its enantioselectivity [170].

The enzymatic synthesis of $\beta$-lactam antibiotics, such as ampicillin, is generally performed under conditions, such as lower $\mathrm{pH}$ and temperature, which are far from the optimum of the enzyme [171]. With the aim of achieving a high synthesis/hydrolysis ratio at high conversion and a high productivity and space-time yield, penicillin $G$ acylase was immobilized in the form of CLEAs using ammonium sulfate or tert-butanol as precipitation agents [132].

For obtaining advanced intermediates of $\beta$-lactam antibiotics such as desacetyl-7-ACA and desacetyl cephalosporanic acid, a highly active CLEA of the purified rAXE from Bacillus pumilus CECT5072 with strong operational stability was produced. The operational stability of this CLEAs was with a half-life of 45 cycles. Therefore, this new methodology should decrease the industrial cost of CLEAs both in terms of biocatalyst production and reusability [133].

Penicillin G acylase (penicillin amidohydrolase; E.C. 3.5.1.11) is widely used in the industrial synthesis of 6-aminopenicillanic acid (6-APA) by the enzymatic deacylation of penicillin $G$ $[172,173]$. The free enzyme is known to have a limited thermal stability and a low tolerance toward organic solvents [174] and this drawback could be circumvented by the immobilization of the enzyme. In the synthesis of ampicillin, CLEAs of penicillin G acylase were more efficient catalysts than cross-linked crystals (CLECs) of the same enzyme and maintained their activity in organic solvents [175].

It is well known that lipases are the most commonly used enzymes in industrial scale. Esterification reactions to produce industrially important products such as emulsifiers, surfactants, wax esters, biopolymers, modified fats and oils, structured lipids, and flavoresters [146], esters of short-chain alcohols and short-chain fatty acids, which are important aroma compounds [170], esters of short-chain alcohols and long-chain fatty acids, which are valuable oleochemicals that may be used as lubricants, diesel fuel, and antistatic reagents [138], and esters of long-chain fatty acids and polyhydric alcohols such as glycerol, sorbitol, and other carbohydrates, can be catalyzed by lipases. Therefore, there exist much interest in their immobilization methods. CLEAs from Candida rugosa lipase $(\mathrm{CrL})$ using glutaraldehyde as the cross-linker had some advantages, such as thermal stability, good reusability and enhanced 
stability, and better dispersibility in organic solvents, which were reflected in improved operational performance compared to the free enzyme in the synthesis of esters from a variety of alcohols and fatty acids [138].

The third generation of biodiesel from microalgae is interesting due to the advantages of easy cultivation and fast reproduction, high oil content, no competition for water and land resources, and being more ecofriendly [176]. PEL-CLEAs catalyzed biodiesel production from microalgal oil in the IL $[\mathrm{BMIm}]\left[\mathrm{PF}_{6}\right]$ with a conversion of $85.7 \%$, demonstrating that they can be taken as a promising catalyst for this application [140].

CLEAs may be used in the environmental care as well. Mushroom tyrosinase was used for the enzymatic treatment of phenolic wastewater. Catalyzed by the enzyme immobilized in the form of CLEAs, phenolic compounds such as phenol, p-cresol, p-chlorophenol, and bisphenol A can be efficiently eliminated, with a complete conversion, superior to other processes catalyzed by the same enzyme [177].

CLEAs of laccase are promising biocatalysts for the decolorization of synthetic dyes in aqueous solution [178]. Namely, industrial applications of laccase for dye decolorization require large amounts of readily available crude or purified laccase, enzyme stability under operational conditions, and recyclability $[1,128,179]$, which can be achieved by enzyme immobilization in CLEA formation. The use of free laccases in such applications has disadvantages, such as their relatively short lifetime and their instability under harsh environment (e.g., temperature, organic solvents, and salts) [4].

In the case of removal of hydrogen peroxide residue from milk, production of gluconicacid, phenyl pyruvic acid, and dihydroxyacetone phosphate catalase has been used in combination with oxidases [180-184]. However, the single use and the instability of free catalase in harsh reaction conditions are not economically viable. The reusability of catalase plays an important role in the industrial applications and can be achieved by immobilization. Therefore, bovine liver catalase was immobilized as cross-linked catalase aggregates (CLEA-CAT) via precipitation with ammonium sulfate and then cross-linking with glutaraldehyde. Finally, BSA as the proteic feeder was used. Although the catalase had the low Vmax value after immobilization process, the thermal and storage stabilities of CLEA-CAT-BSA derivative were comparably higher than those of free CAT. CLEA-CAT-BSA derivative was reused for 400 cycles in the batch-type reactor and the total amount of $\mathrm{H}_{2} \mathrm{O}_{2}$ decomposed was $1.2 \times 10^{4}$ mol $\mathrm{H}_{2} \mathrm{O}_{2}$ throughout 400 consecutive uses [185].

There are also some unusual examples of CLEA use, such as biocatalytic synthesis of silver nanoparticles from silver nitrate. For this biocatalytic synthesis, immobilized NADH-dependent nitrate reductase as CLEAs was used based on the bioreduction ability of nitrate reductase [186]. At the same time, immobilization improved the thermal stability of the enzyme.

Another advantage of this type of immobilized enzymes is the preparation of combined CLEAs, so called combi-CLEAs, which is the possibility to coimmobilize two or more different enzymes in a single CLEA. In this case, CLEAs are capable to catalyze two or more biotransformations, independently or in sequence as catalytic cascade processes.

A good example is the bioconversion of sucrose to trehalose (Figure 9). 


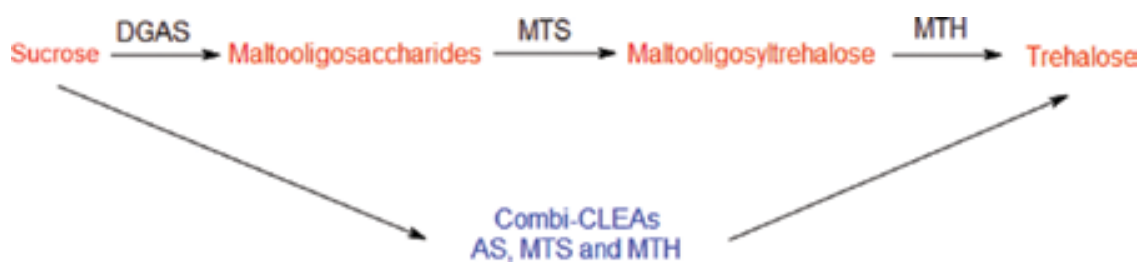

Figure 9. Biotransformation of sucrose to trehalose using combi-CLEAs in comparison to the use of individual enzymes for each reaction step.

Combi-CLEAs of amylosucrase (AS), maltooligosyltrehalose synthase (MTS), and maltooligosyltrehalose trehalohydrolase (MTH) were successfully established with acetone and glutaraldehyde (GA) as a precipitant and a cross-linker to achieve the one-step bioconversion of sucrose to trehalose [187]. The stability of this enzyme preparation was quite good, as reusability of about five cycles without any activity loss was obtained. This reaction is important in the food, cosmetic, and pharmaceutical industries, because trehalose is known to have high water-holding activity and this characteristic makes it applicable to the development of additives, stabilizers, and sweeteners [188].

\section{Conclusion}

The stability of enzymes is important for biotechnological applications and can be achieved via immobilization methods. Large amounts of research have been devoted into exploring the synthesis and modification of magnetic nanoparticles. They have been proven to be a successful tool for the immobilization of enzymes, proteins, drugs, and other biologically active molecules because of their biocompatibility, biodegradability, colloidal stability, low toxicity, and nanometer scale. Magnetic nanoparticles that are properly surface functionalized can be applied into various fields of science, mostly benefiting in biomedicine and biotechnology, improving targeted drug deliveries of active therapeutic agents, and exploring and developing new strategic applications to improve and increase our development in the future. Another interesting possibility for enzyme immobilization is CLEA formation. With their micrometer size and unique advantages, where there is no need for carriers and pure enzymes, they are applicable in different kinds of bioreactors and biosensors.

\section{Acknowledgements}

The authors would like to thank the Slovenian Research Agency (Project "Magnetic nanoparticles as potential carriers of biologically active substances", Contract No. J2-4232) and programme Extraction Processes and Product Design (Contract No. P2-0046). 


\title{
Author details
}

\author{
Maja Leitgeb*, Željko Knez and Katja Vasić \\ *Address all correspondence to: maja.leitgeb@um.si \\ Faculty of Chemistry and Chemical Engineering, University of Maribor, Laboratory for \\ Separation Processes and Product Design, Maribor, Slovenia
}

\section{References}

[1] C. Mateo, J.M. Palomo, G. Fernandez-Lorente, J.M. Guisan, R. Fernandez-Lafuente, Improvement of enzyme activity, stability and selectivity via immobilization techniques, Enzyme Microb. Technol. 40 (2007) 1451-1463. doi:10.1016/j.enzmictec. 2007.01.018.

[2] R.L. Goldberg, L.M. Kolibas, An improved method for determining proteoglycans synthesized by chondrocytes in culture, Connect. Tissue Res. 24 (1990) 265-275.

[3] P.V. Iyer, L. Ananthanarayan, Enzyme stability and stabilization-Aqueous and nonaqueous environment, Process Biochem. 43 (2008) 1019-1032. doi:10.1016/j.procbio. 2008.06.004.

[4] D. Brady, J. Jordaan, Advances in enzyme immobilisation, Biotechnol. Lett. 31 (2009) 1639-1650. doi:10.1007/s10529-009-0076-4.

[5] V. Stepankova, S. Bidmanova, T. Koudelakova, Z. Prokop, R. Chaloupkova, J. Damborsky, Strategies for stabilization of enzymes in organic solvents, ACS Catal. 3 (2013) 2823-2836. doi:10.1021/cs400684x.

[6] M. Inès, G. Dhouha, Glycolipid biosurfactants: Potential related biomedical and biotechnological applications, Carbohydr. Res. 416 (2015) 59-69. doi:10.1016/j.carres. 2015.07.016.

[7] Y. Kuthati, R.K. Kankala, C.-H. Lee, Layered double hydroxide nanoparticles for biomedical applications: Current status and recent prospects, Appl. Clay Sci. 112-113 (2015) 100-116. doi:10.1016/j.clay.2015.04.018.

[8] C. Wang, L. Huang, S. Song, B. Saif, Y. Zhou, C. Dong, et al., Targeted delivery and pHresponsive release of stereoisomeric anti-cancer drugs using $\beta$-cyclodextrin assembled $\mathrm{Fe}_{3} \mathrm{O}_{4}$ nanoparticles, Appl. Surf. Sci. 357, Part B (2015) 2077-2086. doi:10.1016/ j.apsusc.2015.09.189.

[9] M. Löhr, P. Müller, P. Karle, J. Stange, S. Mitzner, R. Jesnowski, et al., Targeted chemotherapy by intratumor injection of encapsulated cells engineered to produce 
CYP2B1, an ifosfamide activating cytochrome P450, Gene Ther. 5 (1998) 1070-1078. doi:10.1038/sj.gt.3300671.

[10] T.K. Jain, M.K. Reddy, M.A. Morales, D.L. Leslie-Pelecky, V. Labhasetwar, Biodistribution, clearance, and biocompatibility of iron oxide magnetic nanoparticles in rats, Mol. Pharm. 5 (2008) 316-327. doi:10.1021/mp7001285.

[11] H. Markides, M. Rotherham, A.J. El Haj, Biocompatibility and toxicity of magnetic nanoparticles in regenerative medicine, J. Nanomater. 2012 (2012) e614094. doi: $10.1155 / 2012 / 614094$.

[12] C. Taze, I. Panetas, S. Kalogiannis, K. Feidantsis, G.P. Gallios, G. Kastrinaki, et al., Toxicity assessment and comparison between two types of iron oxide nanoparticles in Mytilus galloprovincialis, Aquat. Toxicol. 172 (2016) 9-20. doi:10.1016/j.aquatox. 2015.12.013.

[13] M. Leitgeb, K. Heržič, G.H. Podrepšek, A. Hojski, A. Crnjac, Z. Knez, Toxicity of magnetic chitosan micro and nanoparticles as carriers for biologically active substances, Acta Chim. Slov. 61 (2014) 145-152.

[14] G. Liu, J. Gao, H. Ai, X. Chen, Applications and potential toxicity of magnetic iron oxide nanoparticles, Small. 9 (2013) 1533-1545. doi:10.1002/smll.201201531.

[15] C. Gerday, M. Aittaleb, M. Bentahir, J.-P. Chessa, P. Claverie, T. Collins, et al., Coldadapted enzymes: From fundamentals to biotechnology, Trends Biotechnol. 18 (2000) 103-107. doi:10.1016/S0167-7799(99)01413-4.

[16] R.W. Bradley, B. Wang, Designer cell signal processing circuits for biotechnology, N. Biotechnol. 32 (2015) 635-643. doi:10.1016/j.nbt.2014.12.009.

[17] D.B. Kell, N. Swainston, P. Pir, S.G. Oliver, Membrane transporter engineering in industrial biotechnology and whole cell biocatalysis, Trends Biotechnol. 33 (2015) 237246. doi:10.1016/j.tibtech.2015.02.001.

[18] S. Joshi, T. Satyanarayana, In vitro engineering of microbial enzymes with multifarious applications: Prospects and perspectives, Bioresour. Technol. 176 (2015) 273-283. doi:10.1016/j.biortech.2014.10.151.

[19] O.L. Tavano, Protein hydrolysis using proteases: An important tool for food biotechnology, J. Mol. Catal. B Enzym. 90 (2013) 1-11. doi:10.1016/j.molcatb.2013.01.011.

[20] J.E. Hyeon, S.D. Jeon, S.O. Han, Cellulosome-based, Clostridium-derived multifunctional enzyme complexes for advanced biotechnology tool development: Advances and applications, Biotechnol. Adv. 31 (2013) 936-944. doi:10.1016/j.biotechadv.2013.03.009.

[21] G. Yu, J.N. Rosenberg, M.J. Betenbaugh, G.A. Oyler, Pac-Man for biotechnology: Coopting degrons for targeted protein degradation to control and alter cell function, Curr. Opin. Biotechnol. 36 (2015) 199-204. doi:10.1016/j.copbio.2015.08.023. 
[22] V.F. Wendisch, Molecular biotechnology: From enzymes and metabolically engineered microbes to superior and sustainable products and processes, J. Biotechnol. 201 (2015) 1. doi:10.1016/j.jbiotec.2015.02.010.

[23] K.T. Schomburg, I. Ardao, K. Götz, F. Rieckenberg, A. Liese, A.-P. Zeng, et al., Computational biotechnology: Prediction of competitive substrate inhibition of enzymes by buffer compounds with protein-ligand docking, J. Biotechnol. 161 (2012) 391-401. doi:10.1016/j.jbiotec.2012.08.002.

[24] K. Chatterjee, S. Sarkar, K. Jagajjanani Rao, S. Paria, Core/shell nanoparticles in biomedical applications, Adv. Colloid Interface Sci. 209 (2014) 8-39. doi:10.1016/j.cis. 2013.12.008.

[25] M. Fairhead, L. Thöny-Meyer, Bacterial tyrosinases: Old enzymes with new relevance to biotechnology, N. Biotechnol. 29 (2012) 183-191. doi:10.1016/j.nbt.2011.05.007.

[26] Y.Q. Zhang, J. Zhu, R.A. Gu, Improved biosensor for glucose based on glucose oxidaseimmobilized silk fibroin membrane, Appl. Biochem. Biotechnol. 75 (1998) 215-233.

[27] P.C. Pandey, S. Upadhyay, H.C. Pathak, A new glucose biosensor based on sandwich configuration of organically modified sol-gel glass, Electroanalysis. 11 (1999) 59-64. doi:10.1002/(SICI)1521-4109(199901)11:1<59::AID-ELAN59>3.0.CO;2-P.

[28] D.-M. Zhou, H.-X. Ju, H.-Y. Chen, A miniaturized glucose biosensor based on the coimmobilization of glucose oxidase and ferrocene perchlorate in nafion at a microdisk platinum electrode, Sens. Actuators B Chem. 40 (1997) 89-94. doi:10.1016/S09254005(97)80245-6.

[29] G. Marko-Varga, R. Appelqvist, L. Gorton, A glucose sensor based on glucose dehydrogenase adsorbed on a modified carbon electrode, Anal. Chim. Acta. 179 (1986) 371379. doi:10.1016/S0003-2670(00)84481-5.

[30] R. Appelqvist, G. Marko-Varga, L. Gorton, A. Torstensson, G. Johansson, Enzymatic determination of glucose in a flow system by catalytic oxidation of the nicotinamide coenzyme at a modified electrode, Anal. Chim. Acta. 169 (1985) 237-247. doi:10.1016/ S0003-2670(00)86226-1.

[31] S.M. Reddy, P.M. Vadgama, Ion exchanger modified PVC membranes-Selectivity studies and response amplification of oxalate and lactate enzyme electrodes, Biosens. Bioelectron. 12 (1997) 1003-1012. doi:10.1016/S0956-5663(97)00055-9.

[32] J.R. Fernandes, G. de O. Neto, L.T. Kubota, M. Tubino, Use of sorghum seed tissue as a biocatalyst in a stirred reactor for oxalic acid determination, Anal. Commun. 33 (1996) 397-399. doi:10.1039/AC9963300397.

[33] C. Eggenstein, M. Borchardt, C. Diekmann, B. Gründig, C. Dumschat, K. Cammann, et al., A disposable biosensor for urea determination in blood based on an ammoniumsensitive transducer, Biosens. Bioelectron. 14 (1999) 33-41. doi:10.1016/S09565663(98)00103-1. 
[34] L. Dezhong, G. Kai, C. Kang, N. Lihua, Y. Shouzhuo, Sensitive specialization analysis of urea in human blood by surface acoustic wave urea sensor system, Microchem. J. 53 (1996) 6-17. doi:10.1006/mchj.1996.0002.

[35] Q. Li, S. Zhang, J. Yu, Immobilization of L-glutamate oxidase and peroxidase for glutamate determination in flow injection analysis system, Appl. Biochem. Biotechnol. 59 (1996) 53-61. doi:10.1007/BF02787857.

[36] S.A.M. Marzouk, V.V. Cosofret, R.P. Buck, H. Yang, W.E. Cascio, S.S.M. Hassan, Amperometric monitoring of lactate accumulation in rabbit ischemic myocardium, Talanta. 44 (1997) 1527-1541. doi:10.1016/S0039-9140(96)02175-3.

[37] J. Wang, E. Dempsey, M. Ozsoz, M.R. Smyth, Amperometric enzyme electrode for theophylline, The Analyst. 116 (1991) 997-999.

[38] H. Kinoshita, M. Torimura, K. Kano, T. Ikeda, Peroxidase-based amperometric sensor of hydrogen peroxide generated in oxidase reaction: Application to creatinine and creatine assay, Electroanalysis. 9 (1997) 1234-1238. doi:10.1002/elan.1140091603.

[39] G.F. Khan, W. Wernet, A highly sensitive amperometric creatinine sensor, Anal. Chim. Acta. 351 (1997) 151-158. doi:10.1016/S0003-2670(97)00362-0.

[40] M.D. Marazuela, B. Cuesta, M.C. Moreno-Bondi, A. Quejido, Free cholesterol fiberoptic biosensor for serum samples with simplex optimization, Biosens. Bioelectron. 12 (1997) 233-240. doi:10.1016/S0956-5663(97)85341-9.

[41] M.A.T. Gilmartin, J.P. Hart, Fabrication and characterization of a screen-printed, disposable, amperometric cholesterol biosensor, Analyst. 119 (1994) 2331-2336. doi: 10.1039/AN9941902331.

[42] X. Li, Z. Rosenzweig, A fiber optic sensor for rapid analysis of bilirubin in serum, Anal. Chim. Acta. 353 (1997) 263-273. doi:10.1016/S0003-2670(97)87785-9.

[43] M.M. Vidal, M.H. Gil, I. Delgadillo, J. Alonso, Study of the thermal stability and enzymatic activity of an immobilised enzymatic system for the bilirubin oxidation, Biomaterials. 20 (1999) 757-763. doi:10.1016/S0142-9612(98)00228-2.

[44] I. Petrikovics, K. Hong, G. Omburo, Q.Z. Hu, L. Pei, W.D. McGuinn, et al., Antagonism of paraoxon intoxication by recombinant phosphotriesterase encapsulated within sterically stabilized liposomes, Toxicol. Appl. Pharmacol. 156 (1999) 56-63. doi:10.1006/ taap.1998.8620.

[45] L. Pei, I. Petrikovics, J.L. Way, Antagonism of the lethal effects of paraoxon by carrier erythrocytes containing phosphotriesterase, Fundam. Appl. Toxicol. Off. J. Soc. Toxicol. 28 (1995) 209-214.

[46] C. Lizano, S. Sanz, J. Luque, M. Pinilla, In vitro study of alcohol dehydrogenase and acetaldehyde dehydrogenase encapsulated into human erythrocytes by an electropo- 
ration procedure, Biochim. Biophys. Acta BBA Gen. Subj. 1425 (1998) 328-336. doi: 10.1016/S0304-4165(98)00085-3.

[47] M. Magnani, A. Fazi, F. Mangani, L. Rossi, U. Mancini, Methanol detoxification by enzyme-loaded erythrocytes, Biotechnol. Appl. Biochem. 18 (1993) 217-226. doi: 10.1111/j.1470-8744.1993.tb00268.x.

[48] D.B. Yarosh, A. O'Connor, L. Alas, C. Potten, P. Wolf, Photoprotection by topical DMA repair enzymes: Molecular correlates of clinical studies, Photochem. Photobiol. 69 (1999) 136-140. doi:10.1111/j.1751-1097.1999.tb03265.x.

[49] D. Yarosh, J. Klein, J. Kibitel, L. Alas, A. O'Connor, B. Cummings, et al., Enzyme therapy of xeroderma pigmentosum: Safety and efficacy testing of T4N5 liposome lotion containing a prokaryotic DNA repair enzyme, Photodermatol. Photoimmunol. Photomed. 12 (1996) 122-130.

[50] K. Jørgensen, T. Kiebler, I. Hylander, C. Vermehren, Interaction of a lipid-membrane destabilizing enzyme with PEG-liposomes, Int. J. Pharm. 183 (1999) 21-24. doi:10.1016/ S0378-5173(99)00036-8.

[51] P. Karle, P. Müller, R. Renz, R. Jesnowski, R. Saller, K. von Rombs, et al., Intratumoral injection of encapsulated cells producing an oxazaphosphorine activating cytochrome P450 for targeted chemotherapy, Adv. Exp. Med. Biol. 451 (1998) 97-106.

[52] W.H. Günzburg, P. Karle, R. Renz, B. Salmons, M. Renner, Characterization of a human cell clone expressing cytochrome $P 450$ for safe use in human somatic cell therapy, Ann. N. Y. Acad. Sci. 880 (1999) 326-336. doi:10.1111/j.1749-6632.1999.tb09536.x.

[53] A. Šalić, K. Pindrić, G.H. Podrepšek, M. Leitgeb, B. Zelić, NADH oxidation in a microreactor catalysed by ADH immobilised on $\gamma-\mathrm{Fe}_{2} \mathrm{O}_{3}$ nanoparticles, Green Process. Synth. 2 (2013) 569-578. doi:10.1515/gps-2013-0084.

[54] E.G.C. Neiva, M.M. Oliveira, L.H. Marcolino Jr., A.J.G. Zarbin, Nickel nanoparticles with hcp structure: Preparation, deposition as thin films and application as electrochemical sensor, J. Colloid Interface Sci. 468 (2016) 34-41. doi:10.1016/j.jcis.2016.01.036.

[55] S.Z. Mirahmadi-Zare, A. Allafchian, F. Aboutalebi, P. Shojaei, Y. Khazaie, K. Dormiani, et al., Super magnetic nanoparticles $\mathrm{NiFe}_{2} \mathrm{O}_{4}$, coated with aluminum-nickel oxide solgel lattices to safe, sensitive and selective purification of his-tagged proteins, Protein Expr. Purif. 121 (2016) 52-60. doi:10.1016/j.pep.2016.01.008.

[56] P.C. Pandey, D. Panday, Novel synthesis of nickel-iron hexacyanoferrate nanoparticles and its application in electrochemical sensing, J. Electroanal. Chem. 763 (2016) 6370. doi:10.1016/j.jelechem.2015.12.048.

[57] J.F. Blandez, I. Esteve-Adell, A. Primo, M. Alvaro, H. García, Nickel nanoparticles supported on graphene as catalysts for aldehyde hydrosilylation, J. Mol. Catal. Chem. 412 (2016) 13-19. doi:10.1016/j.molcata.2015.11.011. 
[58] M.L. López-Moreno, L.L. Avilés, N.G. Pérez, B.Á. Irizarry, O. Perales, Y. CedenoMattei, et al., Effect of cobalt ferrite $\left(\mathrm{CoFe}_{2} \mathrm{O}_{4}\right)$ nanoparticles on the growth and development of Lycopersicon lycopersicum (tomato plants), Sci. Total Environ. 550 (2016) 45-52. doi:10.1016/j.scitotenv.2016.01.063.

[59] A. Contino, G. Maccarrone, M. Zimbone, R. Reitano, P. Musumeci, L. Calcagno, et al., Tyrosine capped silver nanoparticles: A new fluorescent sensor for the quantitative determination of copper(II) and cobalt(II) ions, J. Colloid Interface Sci. 462 (2016) 216222. doi:10.1016/j.jcis.2015.10.008.

[60] T. Tsoncheva, I. Genova, D. Paneva, M. Dimitrov, B. Tsyntsarski, N. Velinov, et al., Cobalt- and iron-based nanoparticles hosted in SBA-15 mesoporous silica and activated carbon from biomass: Effect of modification procedure, Solid State Sci. 48 (2015) 286293. doi:10.1016/j.solidstatesciences.2015.09.001.

[61] F. Zeb, A.R. Qureshi, K. Nadeem, M. Mumtaz, H. Krenn, Surface effects in uncoated and amorphous $\mathrm{SiO}_{2}$ coated cobalt ferrite nanoparticles, J. Non-Cryst. Solids. 435 (2016) 69-75. doi:10.1016/j.jnoncrysol.2016.01.003.

[62] O.A. Yeshchenko, I.M. Dmitruk, A.A. Alexeenko, A.M. Dmytruk, Optical properties of sol-gel fabricated $\mathrm{Ni} / \mathrm{SiO}_{2}$ glass nanocomposites, J. Phys. Chem. Solids. 69 (2008) 16151622. doi:10.1016/j.jpcs.2007.12.002.

[63] J. Gao, H. Gu, B. Xu, Multifunctional magnetic nanoparticles: Design, synthesis, and biomedical applications, Acc. Chem. Res. 42 (2009) 1097-1107. doi:10.1021/ar9000026.

[64] A.H. Silva, E. Lima Jr., M.V. Mansilla, R.D. Zysler, H. Troiani, M.L.M. Pisciotti, et al., Superparamagnetic iron-oxide nanoparticles mPEG350- and mPEG2000-coated: Cell uptake and biocompatibility evaluation, Nanomedicine Nanotechnol. Biol. Med. doi: 10.1016/j.nano.2015.12.371.

[65] V.I. Shubayev, T.R. Pisanic II, S. Jin, Magnetic nanoparticles for theragnostics, Adv. Drug Deliv. Rev. 61 (2009) 467-477. doi:10.1016/j.addr.2009.03.007.

[66] W. Cheng, X. Xu, F. Wu, J. Li, Synthesis of cavity-containing iron oxide nanoparticles by hydrothermal treatment of colloidal dispersion, Mater. Lett. 164 (2016) 210-212. doi:10.1016/j.matlet.2015.10.170.

[67] E. Fazio, M. Santoro, G. Lentini, D. Franco, S.P.P. Guglielmino, F. Neri, Iron oxide nanoparticles prepared by laser ablation: Synthesis, structural properties and antimicrobial activity, Colloids Surf. Physicochem. Eng. Asp. 490 (2016) 98-103. doi:10.1016/ j.colsurfa.2015.11.034.

[68] M. Abbasi, R. Amiri, A.-K. Bordbar, E. Ranjbakhsh, A.-R. Khosropour, Improvement of the stability and activity of immobilized glucose oxidase on modified iron oxide magnetic nanoparticles, Appl. Surf. Sci. 364 (2016) 752-757. doi:10.1016/j.apsusc. 2015.12.120. 
[69] S.L. Easo, P.V. Mohanan, Dextran stabilized iron oxide nanoparticles: Synthesis, characterization and in vitro studies, Carbohydr. Polym. 92 (2013) 726-732. doi:10.1016/ j.carbpol.2012.09.098.

[70] J. Hradil, A. Pisarev, M. Babič, D. Horák, Dextran-modified iron oxide nanoparticles, China Particuology. 5 (2007) 162-168. doi:10.1016/j.cpart.2007.01.003.

[71] G. Li, Z. Zhou, Y. Li, K. Huang, M. Zhong, Surface functionalization of chitosan-coated magnetic nanoparticles for covalent immobilization of yeast alcohol dehydrogenase from Saccharomyces cerevisiae, J. Magn. Magn. Mater. 322 (2010) 3862-3868. doi:10.1016/ j.jmmm.2010.08.008.

[72] F. Šulek, M. Drofenik, M. Habulin, Ž. Knez, Surface functionalization of silica-coated magnetic nanoparticles for covalent attachment of cholesterol oxidase, J. Magn. Magn. Mater. 322 (2010) 179-185. doi:10.1016/j.jmmm.2009.07.075.

[73] M. Ziegler-Borowska, T. Siódmiak, D. Chełminiak, A. Cyganiuk, M.P. Marszałł, Magnetic nanoparticles with surfaces modified with chitosan-poly[N-benzyl-2(methacryloxy)-N,N-dimethylethanaminium bromide] for lipase immobilization, Appl. Surf. Sci. 288 (2014) 641-648. doi:10.1016/j.apsusc.2013.10.088.

[74] H.-J. Chen, Z.-H. Zhang, L.-J. Luo, S.-Z. Yao, Surface-imprinted chitosan-coated magnetic nanoparticles modified multi-walled carbon nanotubes biosensor for detection of bovine serum albumin, Sens. Actuators B Chem. 163 (2012) 76-83. doi: 10.1016/j.snb.2012.01.010.

[75] R. Khatiri, A. Reyhani, S.Z. Mortazavi, M. Hossainalipour, Immobilization of serum albumin on the synthesized three layers core-shell structures of super-paramagnetic iron oxide nanoparticles, J. Ind. Eng. Chem. 19 (2013) 1642-1647. doi:10.1016/j.jiec. 2013.02.002.

[76] W. Wu, Q. He, C. Jiang, Magnetic iron oxide nanoparticles: Synthesis and surface functionalization strategies, Nanoscale Res. Lett. 3 (2008) 397-415. doi:10.1007/s11671008-9174-9.

[77] R. Qiao, C. Yang, M. Gao, Superparamagnetic iron oxide nanoparticles: From preparations to in vivo MRI applications, J. Mater. Chem. 19 (2009) 6274-6293. doi:10.1039/ B902394A.

[78] L.A. Thomas, L. Dekker, M. Kallumadil, P. Southern, M. Wilson, S.P. Nair, et al., Carboxylic acid-stabilised iron oxide nanoparticles for use in magnetic hyperthermia, J. Mater. Chem. 19 (2009) 6529-6535. doi:10.1039/B908187A.

[79] J. Wotschadlo, T. Liebert, T. Heinze, K. Wagner, M. Schnabelrauch, S. Dutz, et al., Magnetic nanoparticles coated with carboxymethylated polysaccharide shellsInteraction with human cells, J. Magn. Magn. Mater. 321 (2009) 1469-1473. doi:10.1016/ j.jmmm.2009.02.069.

[80] M. Creixell, A.P. Herrera, M. Latorre-Esteves, V. Ayala, M. Torres-Lugo, C. Rinaldi, The effect of grafting method on the colloidal stability and in vitro cytotoxicity of 
carboxymethyl dextran coated magnetic nanoparticles, J. Mater. Chem. 20 (2010) 85398547. doi:10.1039/C0JM01504K.

[81] M.P. Herrling, S. Lackner, O. Tatti, G. Guthausen, M. Delay, M. Franzreb, et al., Short and long term biosorption of silica-coated iron oxide nanoparticles in heterotrophic biofilms, Sci. Total Environ. 544 (2016) 722-729. doi:10.1016/j.scitotenv.2015.11.174.

[82] W.-Y. Rho, H.-M. Kim, S. Kyeong, Y.-L. Kang, D.-H. Kim, H. Kang, et al., Facile synthesis of monodispersed silica-coated magnetic nanoparticles, J. Ind. Eng. Chem. 20 (2014) 2646-2649. doi:10.1016/j.jiec.2013.12.014.

[83] C. Barrera, A. Herrera, Y. Zayas, C. Rinaldi, Surface modification of magnetite nanoparticles for biomedical applications, J. Magn. Magn. Mater. 321 (2009) 1397-1399. doi:10.1016/j.jmmm.2009.02.046.

[84] Ž.K. Gordana Hojnik Podrepšek, Different preparation methods and characterization of magnetic maghemite coated with chitosan, J. Nanoparticle Res. 15 (2013). doi: 10.1007/s11051-013-1751-x.

[85] G. Hojnik Podrepšek, Synthesis comparison and characterization of chitosan-coated magnetic nanoparticles prepared with different methods.

[86] N. Öztürk, S. Akgöl, M. Arısoy, A. Denizli, Reversible adsorption of lipase on novel hydrophobic nanospheres, Sep. Purif. Technol. 58 (2007) 83-90. doi:10.1016/j.seppur. 2007.07.037.

[87] T. Valdés-Solís, A.F. Rebolledo, M. Sevilla, P. Valle-Vigón, O. Bomatí-Miguel, A.B. Fuertes, et al., Preparation, characterization, and enzyme immobilization capacities of superparamagnetic silica/iron oxide nanocomposites with mesostructured porosity, Chem. Mater. 21 (2009) 1806-1814. doi:10.1021/cm8005937.

[88] E.Z. Ramos, R.H.M. Júnior, P.F. de Castro, P.W. Tardioli, A.A. Mendes, R. FernandézLafuente, et al., Production and immobilization of Geotrichum candidum lipase via physical adsorption on eco-friendly support: Characterization of the catalytic properties in hydrolysis and esterification reactions, J. Mol. Catal. B Enzym. 118 (2015) 43-51. doi:10.1016/j.molcatb.2015.05.009.

[89] J. Wu, L. Zhou, H. Zhang, J. Guo, X. Mei, C. Zhang, et al., Direct affinity immobilization of recombinant heparinase I fused to maltose binding protein on maltose-coated magnetic nanoparticles, Biochem. Eng. J. 90 (2014) 170-177. doi:10.1016/j.bej. 2014.05.021.

[90] S. Altun, B. Çakıroğlu, M. Özacar, M. Özacar, A facile and effective immobilization of glucose oxidase on tannic acid modified $\mathrm{CoFe}_{2} \mathrm{O}_{4}$ magnetic nanoparticles, Colloids Surf. B Biointerfaces. 136 (2015) 963-970. doi:10.1016/j.colsurfb.2015.10.053.

[91] F. He, S. Zhang, X. Liu, Immobilization of feruloyl esterases on magnetic nanoparticles and its potential in production of ferulic acid, J. Biosci. Bioeng. 120 (2015) 330-334. doi:10.1016/j.jbiosc.2015.01.006. 
[92] M.D. Joshi, G. Sidhu, I. Pot, G.D. Brayer, S.G. Withers, L.P. McIntosh, Hydrogen bonding and catalysis: A novel explanation for how a single amino acid substitution can change the ph optimum of a glycosidase, J. Mol. Biol. 299 (2000) 255-279. doi: 10.1006/jmbi.2000.3722.

[93] E. Dumitriu, F. Secundo, J. Patarin, I. Fechete, Preparation and properties of lipase immobilized on MCM-36 support, J. Mol. Catal. B Enzym. 22 (2003) 119-133. doi: 10.1016/S1381-1177(03)00015-8.

[94] Y. Zhang, J. Li, D. Han, H. Zhang, P. Liu, C. Li, An efficient resolution of racemic secondary alcohols on magnetically separable biocatalyst, Biochem. Biophys. Res. Commun. 365 (2008) 609-613. doi:10.1016/j.bbrc.2007.10.205.

[95] A.K. Johnson, A.M. Zawadzka, L.A. Deobald, R.L. Crawford, A.J. Paszczynski, Novel method for immobilization of enzymes to magnetic nanoparticles, J. Nanoparticle Res. 10 (2007) 1009-1025. doi:10.1007/s11051-007-9332-5.

[96] S.F. Motevalizadeh, M. Khoobi, A. Sadighi, M. Khalilvand-Sedagheh, M. Pazhouhandeh, A. Ramazani, et al., Lipase immobilization onto polyethylenimine coated magnetic nanoparticles assisted by divalent metal chelated ions, J. Mol. Catal. B Enzym. 120 (2015) 75-83. doi:10.1016/j.molcatb.2015.06.013.

[97] R. Cui, C. Bai, Y. Jiang, M. Hu, S. Li, Q. Zhai, Well-defined bioarchitecture for immobilization of chloroperoxidase on magnetic nanoparticles and its application in dye decolorization, Chem. Eng. J. 259 (2015) 640-646. doi:10.1016/j.cej.2014.08.074.

[98] T. Chen, W. Yang, Y. Guo, R. Yuan, L. Xu, Y. Yan, Enhancing catalytic performance of $\beta$-glucosidase via immobilization on metal ions chelated magnetic nanoparticles, Enzyme Microb. Technol. 63 (2014) 50-57. doi:10.1016/j.enzmictec.2014.05.008.

[99] J. Feng, S. Yu, J. Li, T. Mo, P. Li, Enhancement of the catalytic activity and stability of immobilized aminoacylase using modified magnetic $\mathrm{Fe}_{3} \mathrm{O}_{4}$ nanoparticles, Chem. Eng. J. 286 (2016) 216-222. doi:10.1016/j.cej.2015.10.083.

[100] K. Hernandez, R. Fernandez-Lafuente, Control of protein immobilization: Coupling immobilization and site-directed mutagenesis to improve biocatalyst or biosensor performance, Enzyme Microb. Technol. 48 (2011) 107-122. doi:10.1016/j.enzmictec. 2010.10.003.

[101] D.A. Cowan, R. Fernandez-Lafuente, Enhancing the functional properties of thermophilic enzymes by chemical modification and immobilization, Enzyme Microb. Technol. 49 (2011) 326-346. doi:10.1016/j.enzmictec.2011.06.023.

[102] F. Eslamipour, P. Hejazi, Effects of surface modification and activation of magnetic nanoparticles on the formation of amylase immobilization bonds under different ionic strength conditions, J. Mol. Catal. B Enzym. 119 (2015) 1-11. doi:10.1016/j.molcatb. 2015.05.006. 
[103] J. Huang, H. Liu, P. Zhang, P. Zhang, M. Li, L. Ding, Immobilization of cholesterol oxidase on magnetic fluorescent core-shell-structured nanoparticles, Mater. Sci. Eng. C. 57 (2015) 31-37. doi:10.1016/j.msec.2015.07.038.

[104] A. Xiao, C. Xu, Y. Lin, H. Ni, Y. Zhu, H. Cai, Preparation and characterization of $\kappa^{-}$ carrageenase immobilized onto magnetic iron oxide nanoparticles, Electron. J. Biotechnol. 19 (2016) 1-7. doi:10.1016/j.ejbt.2015.10.001.

[105] T. Saravanakumar, T. Palvannan, D.-H. Kim, S.-M. Park, Optimized immobilization of peracetic acid producing recombinant acetyl xylan esterase on chitosan coated- $\mathrm{Fe}_{3} \mathrm{O}_{4}$ magnetic nanoparticles, Process Biochem. 49 (2014) 1920-1928. doi:10.1016/j.procbio. 2014.08.008.

[106] Y. Sui, Y. Cui, Y. Nie, G.-M. Xia, G.-X. Sun, J.-T. Han, Surface modification of magnetite nanoparticles using gluconic acid and their application in immobilized lipase, Colloids Surf. B Biointerfaces. 93 (2012) 24-28. doi:10.1016/j.colsurfb.2011.11.054.

[107] G.K. Kouassi, J. Irudayaraj, G. McCarty, Activity of glucose oxidase functionalized onto magnetic nanoparticles, Biomagn. Res. Technol. 3 (2005) 1. doi:10.1186/1477-044X-3-1.

[108] A. Panek, O. Pietrow, J. Synowiecki, P. Filipkowski, Immobilization on magnetic nanoparticles of the recombinant trehalose synthase from Deinococcus geothermalis, Food Bioprod. Process. 91 (2013) 632-637. doi:10.1016/j.fbp.2013.04.007.

[109] T. Siódmiak, M. Ziegler-Borowska, M.P. Marszałł, Lipase-immobilized magnetic chitosan nanoparticles for kinetic resolution of $(R, S)$-ibuprofen, J. Mol. Catal. B Enzym. 94 (2013) 7-14. doi:10.1016/j.molcatb.2013.04.008.

[110] S. Kumar, A.K. Jana, I. Dhamija, Y. Singla, M. Maiti, Preparation, characterization and targeted delivery of serratiopeptidase immobilized on amino-functionalized magnetic nanoparticles, Eur. J. Pharm. Biopharm. 85 (2013) 413-426. doi:10.1016/j.ejpb. 2013.06.019.

[111] S. Qian, C. Wang, H. Wang, F. Yu, C. Zhang, H. Yu, Synthesis and characterization of surface-functionalized paramagnetic nanoparticles and their application to immobilization of $\alpha$-acetolactate decarboxylase, Process Biochem. 50 (2015) 1388-1393. doi: 10.1016/j.procbio.2015.05.012.

[112] C. Beladiya, R.K. Tripathy, P. Bajaj, G. Aggarwal, A.H. Pande, Expression, purification and immobilization of recombinant AiiA enzyme onto magnetic nanoparticles, Protein Expr. Purif. 113 (2015) 56-62. doi:10.1016/j.pep.2015.04.014.

[113] J. Long, X. Li, Z. Wu, E. Xu, X. Xu, Z. Jin, et al., Immobilization of pullulanase onto activated magnetic chitosan/ $/ \mathrm{Fe}_{3} \mathrm{O}_{4}$ nanoparticles prepared by in situ mineralization and effect of surface functional groups on the stability, Colloids Surf. Physicochem. Eng. Asp. 472 (2015) 69-77. doi:10.1016/j.colsurfa.2015.02.038. 
[114] F. Šulek, Ž. Knez, M. Habulin, Immobilization of cholesterol oxidase to finely dispersed silica-coated maghemite nanoparticles based magnetic fluid, Appl. Surf. Sci. 256 (2010) 4596-4600. doi:10.1016/j.apsusc.2010.02.055.

[115] M. Liu, X. Dai, R. Guan, X. Xu, Immobilization of Aspergillus niger xylanase A on $\mathrm{Fe}_{3} \mathrm{O}_{4}$ coated chitosan magnetic nanoparticles for xylooligosaccharide preparation, Catal. Commun. 55 (2014) 6-10. doi:10.1016/j.catcom.2014.06.002.

[116] R.A.E. Venkatanarasimhan Swarnalatha, Immobilization of $\alpha$-amylase on gum acacia stabilized magnetite nanoparticles, an easily recoverable and reusable support, J. Mol. Catal. B Enzym. 96 (2013) 6-13. doi:10.1016/j.molcatb.2013.05.022.

[117] J. Wang, F. Ji, J. Xing, S. Cui, Y. Bao, W. Hao, Lipase immobilization onto the surface of PGMA-b-PDMAEMA-grafted magnetic nanoparticles prepared via atom transfer radical polymerization, Chin. J. Chem. Eng. 22 (2014) 1333-1339. doi:10.1016/j.cjche. 2014.09.029.

[118] P. Xu, G. Zeng, D. Huang, S. Hu, C. Feng, C. Lai, et al., Synthesis of iron oxide nanoparticles and their application in Phanerochaete chrysosporium immobilization for $\mathrm{Pb}$ (II) removal, Colloids Surf. Physicochem. Eng. Asp. 419 (2013) 147-155. doi:10.1016/ j.colsurfa.2012.10.061.

[119] H. Hashemi-Moghaddam, S. Kazemi-Bagsangani, M. Jamili, S. Zavareh, Evaluation of magnetic nanoparticles coated by 5 -fluorouracil imprinted polymer for controlled drug delivery in mouse breast cancer model, Int. J. Pharm. 497 (2016) 228-238. doi:10.1016/ j.ijpharm.2015.11.040.

[120] N.S. Elbialy, M.M. Fathy, W.M. Khalil, Doxorubicin loaded magnetic gold nanoparticles for in vivo targeted drug delivery, Int. J. Pharm. 490 (2015) 190-199. doi:10.1016/ j.ijpharm.2015.05.032.

[121] F. Campanhã Vicentini, L.L.C. Garcia, L.C.S. Figueiredo-Filho, B.C. Janegitz, O. Fatibello-Filho, A biosensor based on gold nanoparticles, dihexadecylphosphate, and tyrosinase for the determination of catechol in natural water, Enzyme Microb. Technol. 84 (2016) 17-23. doi:10.1016/j.enzmictec.2015.12.004.

[122] M.H. Mashhadizadeh, R.P. Talemi, Synergistic effect of magnetite and gold nanoparticles onto the response of a label-free impedimetric hepatitis B virus DNA biosensor, Mater. Sci. Eng. C. 59 (2016) 773-781. doi:10.1016/j.msec.2015.10.082.

[123] S. Li, W. Qiu, X. Zhang, J. Ni, F. Gao, Q. Wang, A high-performance DNA biosensor based on the assembly of gold nanoparticles on the terminal of hairpin-structured probe DNA, Sens. Actuators B Chem. 223 (2016) 861-867. doi:10.1016/j.snb.2015.09.121.

[124] R. Hushiarian, N.A. Yusof, A.H. Abdullah, S.A.A. Ahmad, S.W. Dutse, Facilitating the indirect detection of genomic DNA in an electrochemical DNA biosensor using magnetic nanoparticles and DNA ligase, Anal. Chem. Res. 6 (2015) 17-25. doi:10.1016/ j.ancr.2015.10.004. 
[125] J. Huang, H. Liu, P. Zhang, P. Zhang, M. Li, L. Ding, Immobilization of cholesterol oxidase on magnetic fluorescent core-shell-structured nanoparticles, Mater. Sci. Eng. C. 57 (2015) 31-37. doi:10.1016/j.msec.2015.07.038.

[126] F. Yazdani, B. Fattahi, N. Azizi, Synthesis of functionalized magnetite nanoparticles to use as liver targeting MRI contrast agent, J. Magn. Magn. Mater. 406 (2016) 207-211. doi:10.1016/j.jmmm.2016.01.026.

[127] L. Agiotis, I. Theodorakos, S. Samothrakitis, S. Papazoglou, I. Zergioti, Y.S. Raptis, Magnetic manipulation of superparamagnetic nanoparticles in a microfluidic system for drug delivery applications, J. Magn. Magn. Mater. 401 (2016) 956-964. doi:10.1016/ j.jmmm.2015.10.111.

[128] R. Fernandez-Lafuente, Stabilization of multimeric enzymes: Strategies to prevent subunit dissociation, Enzyme Microb. Technol. 45 (2009) 405-418. doi:10.1016/ j.enzmictec.2009.08.009.

[129] R.A. Sheldon, Cross-linked enzyme aggregates as industrial biocatalysts, Org. Process Res. Dev. 15 (2011) 213-223. doi:10.1021/op100289f.

[130] S. Kumar, U. Mohan, A.L. Kamble, S. Pawar, U.C. Banerjee, Cross-linked enzyme aggregates of recombinant Pseudomonas putida nitrilase for enantioselective nitrile hydrolysis, Bioresour. Technol. 101 (2010) 6856-6858. doi:10.1016/j.biortech. 2010.03.084.

[131] F. Šulek, D.P. Fernández, Ž. Knez, M. Habulin, R.A. Sheldon, Immobilization of horseradish peroxidase as crosslinked enzyme aggregates (CLEAs), Process Biochem. 46 (2011) 765-769. doi:10.1016/j.procbio.2010.12.001.

[132] L. Cao, L.M. van Langen, F. van Rantwijk, R.A. Sheldon, Cross-linked aggregates of penicillin acylase: Robust catalysts for the synthesis of $\beta$-lactam antibiotics, J. Mol. Catal. B Enzym. 11 (2001) 665-670. doi:10.1016/S1381-1177(00)00078-3.

[133] S. Montoro-García, F. Gil-Ortiz, J. Navarro-Fernández, V. Rubio, F. García-Carmona, Á. Sánchez-Ferrer, Improved cross-linked enzyme aggregates for the production of desacetyl $\beta$-lactam antibiotics intermediates, Bioresour. Technol. 101 (2010) 331-336. doi:10.1016/j.biortech.2009.08.016.

[134] L. Zhao, L. Zheng, G. Gao, F. Jia, S. Cao, Resolution of N-(2-ethyl-6-methylphenyl) alanine via cross-linked aggregates of Pseudomonas sp. lipase, J. Mol. Catal. B Enzym. 54 (2008) 7-12. doi:10.1016/j.molcatb.2007.12.001.

[135] G.-W. Zheng, H.-L. Yu, C.-X. Li, J. Pan, J.-H. Xu, Immobilization of Bacillus subtilis esterase by simple cross-linking for enzymatic resolution of dl-menthyl acetate, J. Mol. Catal. B Enzym. 70 (2011) 138-143. doi:10.1016/j.molcatb.2011.02.018.

[136] H. Cabana, J.P. Jones, S.N. Agathos, Preparation and characterization of cross-linked laccase aggregates and their application to the elimination of endocrine disrupting chemicals, J. Biotechnol. 132 (2007) 23-31. doi:10.1016/j.jbiotec.2007.07.948. 
[137] K. Sangeetha, T. Emilia Abraham, Preparation and characterization of cross-linked enzyme aggregates (CLEA) of subtilisin for controlled release applications, Int. J. Biol. Macromol. 43 (2008) 314-319. doi:10.1016/j.ijbiomac.2008.07.001.

[138] F. Kartal, M.H.A. Janssen, F. Hollmann, R.A. Sheldon, A. Kılınc, Improved esterification activity of Candida rugosa lipase in organic solvent by immobilization as crosslinked enzyme aggregates (CLEAs), J. Mol. Catal. B Enzym. 71 (2011) 85-89. doi: 10.1016/j.molcatb.2011.04.002.

[139] B.S. Aytar, U. Bakir, Preparation of cross-linked tyrosinase aggregates, Process Biochem. 43 (2008) 125-131. doi:10.1016/j.procbio.2007.11.001.

[140] J.-Q. Lai, Z.-L. Hu, R.A. Sheldon, Z. Yang, Catalytic performance of cross-linked enzyme aggregates of Penicillium expansum lipase and their use as catalyst for biodiesel production, Process Biochem. 47 (2012) 2058-2063. doi:10.1016/j.procbio. 2012.07.024.

[141] H.W. Yu, H. Chen, X. Wang, Y.Y. Yang, C.B. Ching, Cross-linked enzyme aggregates (CLEAs) with controlled particles: Application to Candida rugosa lipase, J. Mol. Catal. B Enzym. 43 (2006) 124-127. doi:10.1016/j.molcatb.2006.07.001.

[142] R.A. Sheldon, Characteristic features and biotechnological applications of cross-linked enzyme aggregates (CLEAs), Appl. Microbiol. Biotechnol. 92 (2011) 467-477. doi: 10.1007/s00253-011-3554-2.

[143] F. López-Gallego, L. Betancor, C. Mateo, A. Hidalgo, N. Alonso-Morales, G. Dellamora-Ortiz, et al., Enzyme stabilization by glutaraldehyde crosslinking of adsorbed proteins on aminated supports, J. Biotechnol. 119 (2005) 70-75. doi:10.1016/j.jbiotec. 2005.05.021.

[144] S. Shah, A. Sharma, M.N. Gupta, Preparation of cross-linked enzyme aggregates by using bovine serum albumin as a proteic feeder, Anal. Biochem. 351 (2006) 207-213. doi:10.1016/j.ab.2006.01.028.

[145] T. Honda, M. Miyazaki, H. Nakamura, H. Maeda, Facile preparation of an enzymeimmobilized microreactor using a cross-linking enzyme membrane on a microchannel surface, Adv. Synth. Catal. 348 (2006) 2163-2171. doi:10.1002/adsc.200606224.

[146] T. Dong, L. Zhao, Y. Huang, X. Tan, Preparation of cross-linked aggregates of aminoacylase from Aspergillus melleus by using bovine serum albumin as an inert additive, Bioresour. Technol. 101 (2010) 6569-6571. doi:10.1016/j.biortech.2010.03.061.

[147] M.P. Guauque Torres, M.L. Foresti, M.L. Ferreira, Effect of different parameters on the hydrolytic activity of cross-linked enzyme aggregates (CLEAs) of lipase from Thermomyces lanuginosa, Biochem. Eng. J. 72 (2013) 18-23. doi:10.1016/j.bej.2012.12.010.

[148] M. Wang, W. Qi, Q. Yu, R. Su, Z. He, Cross-linking enzyme aggregates in the macropores of silica gel: A practical and efficient method for enzyme stabilization, Biochem. Eng. J. 52 (2010) 168-174. doi:10.1016/j.bej.2010.08.003. 
[149] H. Cabana, J.P. Jones, S.N. Agathos, Utilization of cross-linked laccase aggregates in a perfusion basket reactor for the continuous elimination of endocrine-disrupting chemicals, Biotechnol. Bioeng. 102 (2009) 1582-1592. doi:10.1002/bit.22198.

[150] N. Hilal, R. Nigmatullin, A. Alpatova, Immobilization of cross-linked lipase aggregates within microporous polymeric membranes, J. Membr. Sci. 238 (2004) 131-141. doi:10.1016/j.memsci.2004.04.002.

[151] L. Wilson, A. Illanes, B.C.C. Pessela, O. Abian, R. Fernández-Lafuente, J.M. Guisán, Encapsulation of crosslinked penicillin $\mathrm{G}$ acylase aggregates in lentikats: Evaluation of a novel biocatalyst in organic media, Biotechnol. Bioeng. 86 (2004) 558-562. doi:10.1002/ bit.20107.

[152] M.I. Kim, J. Kim, J. Lee, H. Jia, H.B. Na, J.K. Youn, et al., Crosslinked enzyme aggregates in hierarchically-ordered mesoporous silica: A simple and effective method for enzyme stabilization, Biotechnol. Bioeng. 96 (2007) 210-218. doi:10.1002/bit.21107.

[153] A.R. Toral, A.P. de los Ríos, F.J. Hernández, M.H.A. Janssen, R. Schoevaart, F. van Rantwijk, et al., Cross-linked Candida antarctica lipase B is active in denaturing ionic liquids, Enzyme Microb. Technol. 40 (2007) 1095-1099. doi:10.1016/j.enzmictec. 2006.08.027.

[154] F. van Rantwijk, R. Madeira Lau, R.A. Sheldon, Biocatalytic transformations in ionic liquids, Trends Biotechnol. 21 (2003) 131-138. doi:10.1016/S0167-7799(03)00008-8.

[155] Z. Yang, W. Pan, Ionic liquids: Green solvents for nonaqueous biocatalysis, Enzyme Microb. Technol. 37 (2005) 19-28. doi:10.1016/j.enzmictec.2005.02.014.

[156] R.M. Lau, M.J. Sorgedrager, G. Carrea, F. van Rantwijk, F. Secundo, R.A. Sheldon, Dissolution of Candida antarctica lipase B in ionic liquids: Effects on structure and activity, Green Chem. 6 (2004) 483-487. doi:10.1039/B405693K.

[157] A.J. Walker, N.C. Bruce, Combined biological and chemical catalysis in the preparation of oxycodone, Tetrahedron. 60 (2004) 561-568. doi:10.1016/j.tet.2003.11.063.

[158] T. Hassani, S. Ba, H. Cabana, Formation of enzyme polymer engineered structure for laccase and cross-linked laccase aggregates stabilization, Bioresour. Technol. 128 (2013) 640-645. doi:10.1016/j.biortech.2012.10.058.

[159] J. Mills, K.K. Schmiegel, W.N. Shaw, Phenethanolamines, compositions containing the same and method for effecting weight control, US4845127 A, 1989. http:// www.google.com.ar/patents/US4845127 (accessed February 25, 2016).

[160] Y.B. Tewari, Thermodynamics of industrially-important, enzyme-catalyzed reactions, Appl. Biochem. Biotechnol. 23 (1990) 187-203. doi:10.1007/BF02942054.

[161] M.I. Youshko, L. Van, R.A. Sheldon, V.K. Švedas, Application of aminoacylase I to the enantioselective resolution of $\alpha$-amino acid esters and amides, Tetrahedron Asymmetry. 15 (2004) 1933-1936. doi:10.1016/j.tetasy.2004.05.018. 
[162] A. Liljeblad, L.T. Kanerva, Enzymatic methods for the preparation of enantiopure malic and aspartic acid derivatives in organic solvents, Tetrahedron Asymmetry. 10 (1999) 4405-4415. doi:10.1016/S0957-4166(99)00476-0.

[163] A. Liljeblad, J. Lindborg, L.T. Kanerva, Acylase I in the alcoholysis of $\alpha$-substituted dicarboxylic acid esters and derivatives: Enantio- and regioselectivity, Tetrahedron Asymmetry. 11 (2000) 3957-3966. doi:10.1016/S0957-4166(00)00346-3.

[164] A. Liljeblad, R. Aksela, L.T. Kanerva, Use of enantio-, chemo- and regioselectivity of acylase I. Resolution of polycarboxylic acid esters, Tetrahedron Asymmetry. 12 (2001) 2059-2066. doi:10.1016/S0957-4166(01)00362-7.

[165] B. Herradón, S. Valverde, Biocatalysis in organic synthesis. 6. First use of an acylase as catalyst in the irreversible transacylation of alcohols and amines: Application to selective transformations, Synlett. 1995 (1995) 599-602. doi:10.1055/s-1995-5025.

[166] P. Noheda, G. Garciá, M.C. Pozuelo, B. Herradón, Chemo-enzymatic synthesis of chiral cyclic compounds: Efficient kinetic resolution of 2-bromo-2-cyclohexenol, Tetrahedron Asymmetry. 7 (1996) 2801-2804. doi:10.1016/0957-4166(96)00366-7.

[167] J. Faraldos, E. Arroyo, B. Herradón, Biocatalysis in organic synthesis. 9. Highly enantioselective kinetic resolution of secondary alcohols catalyzed by acylase. Synlett. 1997 (1997) 367-370. doi:10.1055/s-1997-796.

[168] M. Bakker, A.S. Spruijt, R. Van, R.A. Sheldon, Highly enantioselective aminoacylasecatalyzed transesterification of secondary alcohols, Tetrahedron Asymmetry. 11 (2000) 1801-1808. doi:10.1016/S0957-4166(00)00118-X.

[169] M.I. Youshko, R. Van, R.A. Sheldon, Enantioselective acylation of chiral amines catalysed by aminoacylase I, Tetrahedron Asymmetry. 12 (2001) 3267-3271. doi: 10.1016/S0957-4166(01)00563-8.

[170] B.K. Vaidya, S.S. Kuwar, S.B. Golegaonkar, S.N. Nene, Preparation of cross-linked enzyme aggregates of 1-aminoacylase via co-aggregation with polyethyleneimine, J. Mol. Catal. B Enzym. 74 (2012) 184-191. doi:10.1016/j.molcatb.2011.10.003.

[171] L.M. van Langen, E. de Vroom, F. van Rantwijk, R. Sheldon, Enzymatic synthesis of $\beta$ lactam antibiotics using penicillin-G acylase in frozen media, FEBS Lett. 456 (1999) 8992. doi:10.1016/S0014-5793(99)00939-4.

[172] A. Bruggink, E.C. Roos, E. de Vroom, Penicillin acylase in the industrial production of $\beta$-lactam antibiotics, Org. Process Res. Dev. 2 (1998) 128-133. doi:10.1021/op9700643.

[173] J.G. Shewale, V.K. Sudhakaran, Penicillin V acylase: Its potential in the production of 6-aminopenicillanic acid, Enzyme Microb. Technol. 20 (1997) 402-410. doi:10.1016/ S0141-0229(96)00176-7. 
[174] C.M. Rosell, R. Fernandez-Lafuente, J.M. Guisan, Modification of enzyme properties by the use of inhibitors during their stabilisation by multipoint covalent attachment, Biocatal. Biotransformation. 12 (1995) 67-76. doi:10.3109/10242429508998152.

[175] L. Cao, F. van Rantwijk, R.A. Sheldon, Cross-linked enzyme aggregates: A simple and effective method for the immobilization of penicillin acylase, Org. Lett. 2 (2000) 13611364. doi:10.1021/ol005593x.

[176] A.L. Ahmad, N.H.M. Yasin, C.J.C. Derek, J.K. Lim, Microalgae as a sustainable energy source for biodiesel production: A review, Renew. Sustain. Energy Rev. 15 (2011) 584593. doi:10.1016/j.rser.2010.09.018.

[177] D.-Y. Xu, Z. Yang, Cross-linked tyrosinase aggregates for elimination of phenolic compounds from wastewater, Chemosphere. 92 (2013) 391-398. doi:10.1016/j.chemosphere.2012.12.076.

[178] V. Vinoth Kumar, M.P. Prem Kumar, K.V. Thiruvenkadaravi, P. Baskaralingam, P. Senthil Kumar, S. Sivanesan, Preparation and characterization of porous cross linked laccase aggregates for the decolorization of triphenyl methane and reactive dyes, Bioresour. Technol. 119 (2012) 28-34. doi:10.1016/j.biortech.2012.05.078.

[179] V.V. Kumar, V. Sathyaselvabala, M.P. Premkumar, T. Vidyadevi, S. Sivanesan, Biochemical characterization of three phase partitioned laccase and its application in decolorization and degradation of synthetic dyes, J. Mol. Catal. B Enzym. 74 (2012) 6372. doi:10.1016/j.molcatb.2011.08.015.

[180] N.Y. Farkye, Cheese technology, Int. J. Dairy Technol. 57 (2004) 91-98. doi:10.1111/j. 1471-0307.2004.00146.x.

[181] A.M. Amorim, M.D.G. Gasques, J. Andreaus, M. Scharf, The application of catalase for the elimination of hydrogen peroxide residues after bleaching of cotton fabrics, An. Acad. Bras. Ciênc. 74 (2002) 433-436. doi:10.1590/S0001-37652002000300006.

[182] G. Ozyilmaz, S.S. Tukel, Simultaneous co-immobilization of glucose oxidase and catalase in their substrates, Appl. Biochem. Microbiol. 43 (2007) 29-35. doi:10.1134/ S000368380701005X.

[183] L. Krämer, E. Steckhan, Coimmobilization of L- $\alpha$-glycerophosphate oxidase with catalase and its application for the synthesis of dihydroxyacetone phosphate, Tetrahedron. 53 (1997) 14645-14650. doi:10.1016/S0040-4020(97)01009-0.

[184] B. Kondor, J. García-Serna, M. Poliakoff, N.R. Thomas, M.J. Cocero, AIChE Annual Meeting Conference Proceedings, Philadelphia (2008), pp. 16-21.

[185] S.S. Tükel, F. Hürrem, D. Yildirim, Ö. Alptekin, Preparation of crosslinked enzyme aggregates (CLEA) of catalase and its characterization, J. Mol. Catal. B Enzym. 97 (2013) 252-257. doi:10.1016/j.molcatb.2013.09.007.

[186] S. Talekar, G. Joshi, R. Chougle, B. Nainegali, S. Desai, A. Joshi, et al., Preparation of stable cross-linked enzyme aggregates (CLEAs) of NADH-dependent nitrate reduc- 
tase and its use for silver nanoparticle synthesis from silver nitrate, Catal. Commun. 53 (2014) 62-66. doi:10.1016/j.catcom.2014.05.003.

[187] D.-H. Jung, J.-H. Jung, D.-H. Seo, S.-J. Ha, D.-K. Kweon, C.-S. Park, One-pot bioconversion of sucrose to trehalose using enzymatic sequential reactions in combined crosslinked enzyme aggregates, Bioresour. Technol. 130 (2013) 801-804. doi:10.1016/ j.biortech.2012.12.162.

[188] K. Maruta, T. Nakada, M. Kubota, H. Chaen, T. Sugimoto, M. Kurimoto, et al., Formation of trehalose from maltooligosaccharides by a novel enzymatic system, Biosci. Biotechnol. Biochem. 59 (1995) 1829-1834. doi:10.1271/bbb.59.1829. 
Chapter 3

\title{
Construction and Biological Evaluation of Nanoparticle- Based Tumor Targeting Drug Delivery Systems
}

\author{
Hong $\mathrm{Wu}$, Tiehong Yang and Li Fan \\ Additional information is available at the end of the chapter
}

http://dx.doi.org/10.5772/63733

\begin{abstract}
Nanoparticle-based drug delivery systems have gained immense popularity due to their ability to overcome biological barriers, effectively deliver drugs, and preferentially target tissue. In this chapter, the current progresses and challenges, especially evaluation methods for nanodrugs in antitumor drug delivery systems, are summarized, citing our works targeted at cancer therapy. It includes four parts. First, the principle, advantages, and significance of nanoparticle-based tumor targeting drug delivery system are presented. Recent developments in nanoparticle-based tumor targeting drug delivery system including passive targeting, active targeting, and stimuli-responsive systems/triggered release are introduced. Second, current formulations of nanoparticle-based drug delivery systems are described, including lipid-based, polymeric and branched polymeric, metal-based, magnetic, and mesoporous silica. Third, analytical techniques used for evaluating nanodrugs in vitro and in vivo are emphatically described. Finally, disadvantages and challenges of nanodrug are also discussed.
\end{abstract}

Keywords: nanoparticle, nanomicelle, tumor targeting, biological evaluation, nanocarrier, nanodrug, controlled-release, drug delivery system

\section{Introduction}

The last decade has witnessed enormous advances in the development and application of nanotechnology in cancer detection, diagnosis, and therapy. A nanoparticle as per the National Institutes of Health (NIH) guidelines is any material that is used in the formulation of a drug resulting in a final product smaller than 1 micron in size. This chapter summarizes current progresses and challenges, especially evaluation method for nanodrug in antitumor drug 
delivery systems, citing our works targeted at cancer therapy. This chapter mainly consists of four parts. The first part presents the principle, advantages, and significance of nanoparticlebased tumor targeting drug delivery system, including passive targeting, active targeting, and stimuli-responsive systems/triggered release. The second part introduces the formulations of nanocarriers, with emphases laid on lipid-based, polymeric and branched polymeric, metalbased, and mesoporous silica. Some nanodrug carriers designed by us are introduced in this part. They are active targeting and acid-responsible nanoparticles, novel copolymers, multifunctional acid-sensitive micelle, and tumor microenvironment multiple responsible nanodrug release system. The third part introduces analytical techniques used for the characterization of nanoparticles in vitro and in vivo, such as dynamic light scattering (DLS), transmission electron microscope (TEM), scanning electronic microscopy (SEM), NMR, FTIR, and UV-Vis were commonly used to characterize the nanodrugs. Techniques for cell biology, such as TEM, confocal microscopy, flow cytometry, Western blot, and immunohistochemistry (IHC), were employed to evaluate target ability of nanodrugs in vitro. In vivo imaging system, micro-CT, NMR, and drug biodistribution were used to assess the in vivo behavior and efficacy of nanodrugs. Finally, disadvantages and challenges of nanodrug are discussed. So far, there are so many papers but so few nanodrugs in cancer therapy. The uncertainty and limitation of nanodrugs in pharmacology, toxicology, immunology, largescale manufacturing, quality standard setting, and regulatory issues make nanoparticlebased tumor targeting delivery system have a long way to go.

\section{Construction of nanoparticle-based tumor targeting drug delivery systems and their targeting functionalities}

\subsection{Definition of nanoparticle-based drug delivery system and classification of targeting functionalities}

Increasing demand for and awareness of the applications of nanotechnology in medicine has resulted in the emergence of a new fast-growing multidisciplinary area-nanomedicine. Nanoparticles (NPs) serve as promising delivery system for various cargos such as drugs. Drugs are incorporated in nanoparticles that have the ability to get through physiological barriers and access the whole systemic circulation and thus are cleared less rapidly than free drug.

Nanoparticle-based drug delivery system represents an opportunity to achieve sophisticated targeting strategies and multi-functionality. They can increase the antitumor efficacy of conventional chemo-therapeutics, decrease their systemic toxicity, prolong duration time in systemic circulation, also present the following advantages, (1) help to overcome problems of solubility and chemical stability of anti-cancer drugs; (2) protect anti-cancer drug from biodegradation or excretion; (3) help to improve distribution of chemo therapeutics; (4) designed to release their payload response to biological triggers; and (5) may decrease resistance of tumors against anti-cancer drugs. 
Therefore, targeted delivery is of utmost importance in order to overcome current limitations in cancer therapy. Recent developments in nanoparticle-based tumor targeting drug delivery system could be concluded in four aspects, passive targeting, active targeting, and stimuliresponsive systems/triggered release.

\subsection{Passive targeting}

Passive targeting is realized by specific porous loose structure of tumor vessels, which is easier for nanoparticles to accumulate. This leaky cascularization is the so-called EPR effect (enhanced permeability and retention effect), which allows migration of macromolecules up to $400 \mathrm{~nm}$ into tumor site [1-5]. For example, pegylated liposomal doxorubicin (Doxil@/Caelyx®) and nab-paclitaxel (Abraxane ${ }^{\circledR}$ ) are the first generation nanomedicine based on passive targeting [6]. Numerous macromolecules and nanocarriers have shown to accumulate in tumor via the passive targeting owing to the EPR effect [7, 8]. EPR-based chemotherapy is thus becoming an important strategy to improve the delivery of therapeutic agents to tumors for anticancer drug development, and macromolecular agents are potentially usefully for not only cancer therapy, but for cancer diagnosis and imaging [9]. Although passive targeting approaches form the basis of clinical therapy, they suffer from several limitations. Not all the tumors exhibit EPR effects, and the permeability of vessels may not be the same throughout a single tumor [10, 11]. For example, Kaposi sarcoma with fenestrated vasculature, nanomedicine therapeutics could passive target into tumors without any specific ligand attached to the surface of the nanocarrier. However, heterogeneity of the tumor, such as different hypoxic gradient, can severely impact on the efficacy of passive targeting delivery. Moreover, increased interstitial fluid pressure (IFP) is another limitation of passive targeting, which reduces convective transport, while the dense extracellular matrix hinders diffusion [12]. Finally, though passive targeting could be used for delivering nanomedicine to certain solid tumor, it does not prevent accumulation of nanocarriers in some organs with fenestrated endothelium, for example, the liver and spleen [13].

Therefore, the development of nanomedicine drugs with active targeting functionalities is certainly warranted. One way to increase the targeting efficacy of nanoparticle-based drug delivery systems is to attach affinity ligands, such as antibodies [14], peptides [15], aptamers [16] or small molecules such as folic acid and carbohydrates onto the surface.

\subsection{Active targeting}

Passive targeting allows for the efficient localization of nanoparticles within the tumor microenvironment. Active targeting facilitates the active uptake of nanoparticles by the tumor cells themselves. Nanoparticle-based drug delivery systems decorated with specific targeting ligands will recognize and bind to target cells and then enter the cells through receptor mediated endocytosis. In order to achieve high specificity, those receptors should be highly expressed on tumor cells, but not on normal cells. In our previous studies, folic acid [17], LHRH [18], HAb18 F( $\left.\mathrm{ab}^{\prime}\right)_{2}$ [19] and monoclonal antibody [14] have been conjugated on the nanoparticles surface to enhance their targeting efficacy. The active targeting nanoparticles first specific bind to the receptor on the cell surface, then get internalized in small concave formed on the 
cell membrane. Small concave closed the opening to form endocytic vesicle, then early endosome. The newly formed endosome is transferred to specific organelles, and drugs could be released by acidic $\mathrm{pH}$ or enzymes [20-22]. This endocytosis procedure was also confirmed in our recent research [14], which was illustrated by the schematic below (Figure 1).
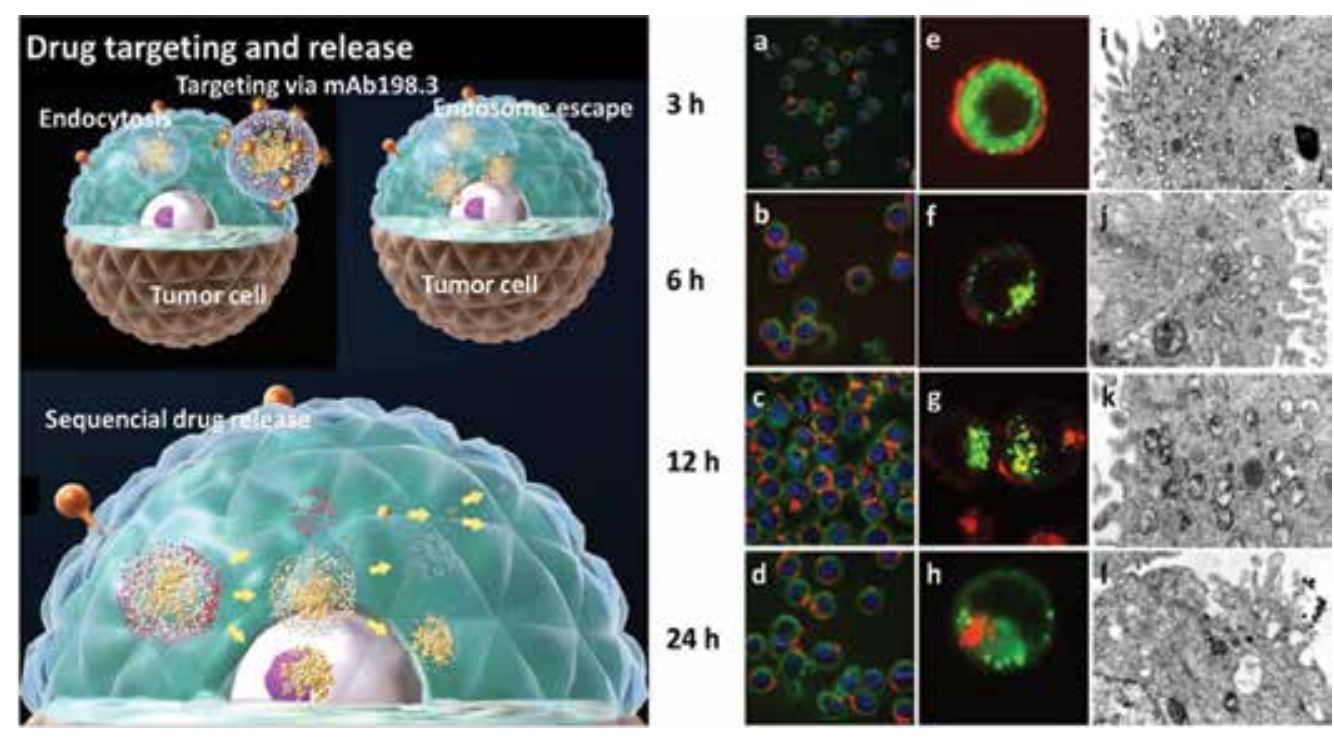

$12 \mathrm{~h}$
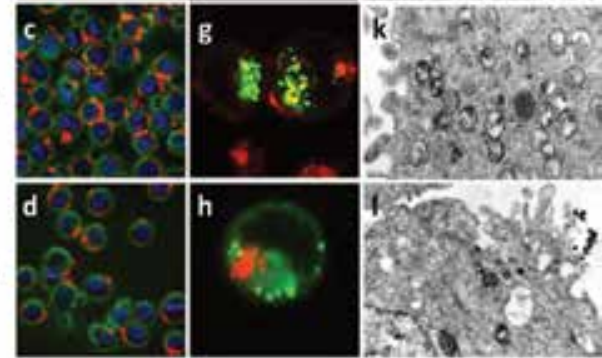

Figure 1. Illustrative schematic representing the endocytosis procedure of the $\mathrm{SiO}_{2} @ \mathrm{AuNP}$ delivery system after binding to cell surface targets. Followed by escaping from the endosomes/lysosomes, the drugs were sequentially released in cytoplasm to eliminate cancer cells. Confocal microscopy and TEM were introduced to testify the endocytosis and endosomal escape procedure of $\mathrm{SiO}_{2} @ \mathrm{AuNP}[14]$.

Among the potential targets for mAb-mediated nanoparticle delivery, human epidermal growth factor receptor 2 (HER2) [23], epidermal growth factor receptor (EGFR) [24], transferrin receptor (TfR) [25], and prostate-specific membrane antigen (PSMA) [26] have been extensively investigated. Over the last several years, aptamers have quickly become a new class of targeting ligands for drug delivery applications. Aptamer-based delivery systems of chemotherapy drugs (e.g., doxorubicin, docetaxel, daunorubicin, and cisplatin), toxins (e.g., gelonin and various photodynamic therapy agents), and a variety of small interfering RNAs were well established during past years [27]. Small molecules such as folic acid were also been widely used due to its inherent properties, which confer distinctive advantages and make it suitable ligand for nanoparticle targeting [28].

Furthermore, active targeting of nanocarriers has shown the potential to suppress multidrug resistance (MDR) via bypassing of P-glycoprotein-mediated drug efflux [29].

Although active targeting delivery systems looks promising, no one was currently approved for clinical use. Moreover, nanodrugs currently under clinical development lack specific targeting. 


\subsection{Stimuli-responsive systems/triggered release}

Although passive and active targeting has been widely investigated, it still cannot guarantee sufficient high drug concentration in tumor site to achieve the complete eradication of tumors. Sufficient and sustained therapy is on the demand of controlled and sustained release of chemotherapeutics in tumor site. Therefore, it is highly desirable to design stimuli-responsive controlled drug delivery systems (CDDSs), which could release drugs by responding to tumor cell environmental changes, such as $\mathrm{pH}$, temperature, glucose, adenosine- 5 '-triphosphate (ATP), glutathione (GSH), and $\mathrm{H}_{2} \mathrm{O}_{2}[30]$.

Among these stimuli, change in acidity as an internal signal is particularly crucial for the development of CDDSs that facilitate tumor targeting. Compared to the extracellular $\mathrm{pH}$ of normal tissues at $\mathrm{pH} 7.4$, the measured tumor extracellular $\mathrm{pH}\left(\mathrm{pH}_{\mathrm{e}}\right)$ values of most solid tumors range from $\mathrm{pH} 6.5$ to 7.2. Moreover, changes in $\mathrm{pH}$ are also encountered once the CDDSs enter cells via endocytosis where $\mathrm{pH}$ can drop as low as 5.0-6.0 in endosomes and 4.05.0 in lysosomes. The $\mathrm{pH}$ gradient is caused by hypoxia that upregulates glycolysis, followed by the production of lactate and protons in extracellular microenvironments [31]. pH-sensitive CDDSs can be used for delivering anti-cancer drugs to specific cancer cells, enhancing cellular internalization and rapid intracellular drug release. In order to increase the targeting activity, ligand-modified $\mathrm{pH}$-sensitive CDDSs have been used for tumor targeting [32,33]. In our previous study, many efforts have been made on several systems based on $\mathrm{pH}$ sensitive drug release characteristics. For instance, (1) pyrrolidinedithiocarbamate (PDTC) and doxorubicin

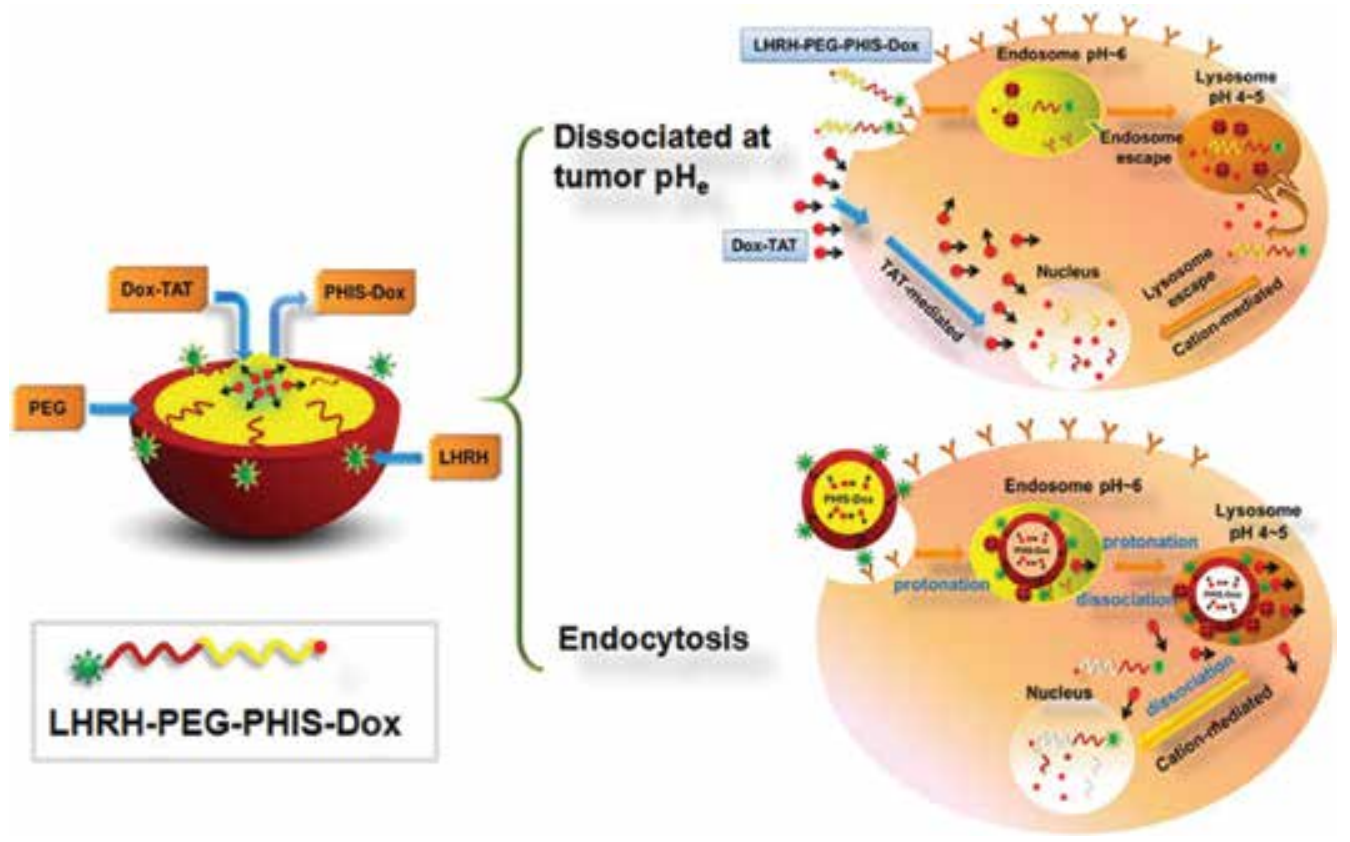

Figure 2. Schematic of targeting approaches and drug release procedures of LHRH-PEG-PHIS-Dox/Dox-TAT mixed micelles [18]. 
(DOX) was codelivered by copolymer folate-chitosan (FA-CS) nanoparticles to achieve targeted drug delivery, stimuli sensitive drug release, and to overcome multidrug resistance (MDR). (2) A novel delivery system based on LHRH-PEG-PHIS-Dox/Dox-TAT acid-sensitive micelles was developed, as shown in Figure 2. Such system could dissociate when responding tumor extracellular pHe and release Dox-TAT. This system showed remarkable antitumor efficacy and negligible systematic toxicity.

Higher concentration of GSH tripeptides is another important internal stimulus for rapid destabilization of CDDSs inside cells to accomplish rapid intracellular release [34]. The intracellular GSH concentration $(1-10 \mathrm{mM})$ is substantially higher than extracellular levels ( $2 \mu \mathrm{M}$ in plasma), providing a mechanism for selective intracellular release [35]. Gold nanoparticles were widely used for design GSH-triggered drug delivery systems. Its surface monolayer is stable under most physiological conditions, thus providing a reservoir of hydrophobic drugs, yet allowing controlled release by GSH though place exchange reactions of thiols on gold nanoparticle surfaces. These Au nanoparticles systems, which are under intensive study, display very intriguing properties, such as the precise control of intracellular drug release triggered by GSH. However, despite their great potential, additional investigations will be required to fully understand their pharmacokinetics, their interactions with the immune system, and the extent of cytotoxicity due to the surface and the geometry of the gold nanoparticles. Our research also focused on GSH-mediated drug release, such as siRNA [14] (Figure 3) and miR-218 mimics [36] (Figure 4) release from AuCOOH. After endocytosis, mediated by mAb198.3, the siRNA release process was illustrated by Figure 3. siRNA was released by the place exchange of glutathione (GSH) [37], and different band shifts on the denatured polyacrylamide gel page demonstrated the process of GSH-triggered siRNA release. In the research based on FA-CS@AuCOOH nanoparticles, temozolomide was released by diffusion due to FA-CS nanogel swelling, followed by miR-218 mimics was released by place exchange of GSH in tumor cells, which was illustrated in Figure 4. The sequential release of both chemo-drug and bio-drug exhibited significant synergistic effect against U87MG glioblastoma cells.
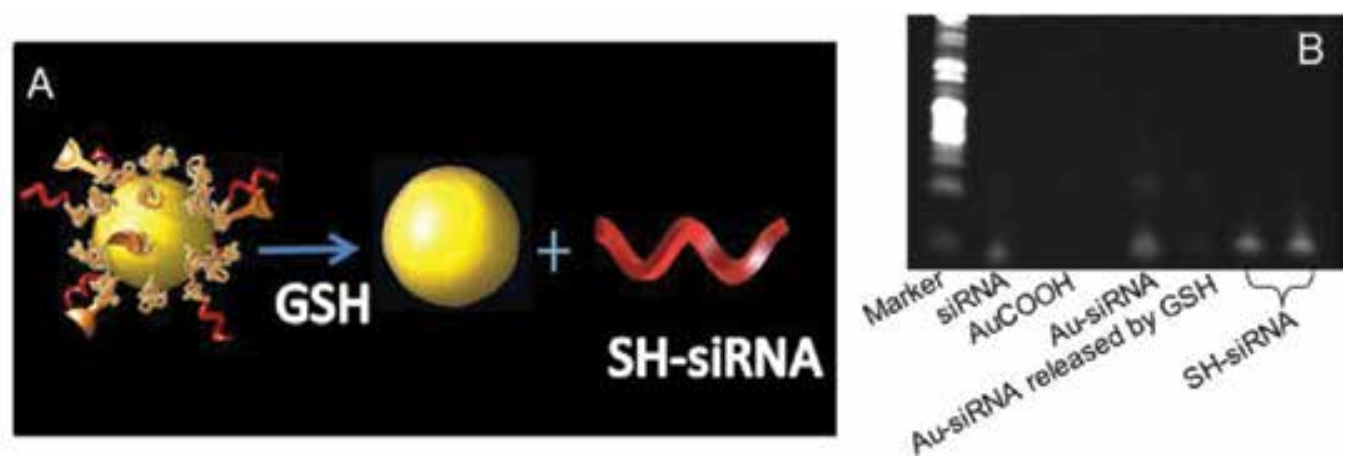

Figure 3. siRNA release procedure of outer AuNP layer. Schematic illustration of siRNA release procedures via GSH place exchange (A), confirmed by denatured SDS page (B) [14]. 


\section{Drug targeting and release}

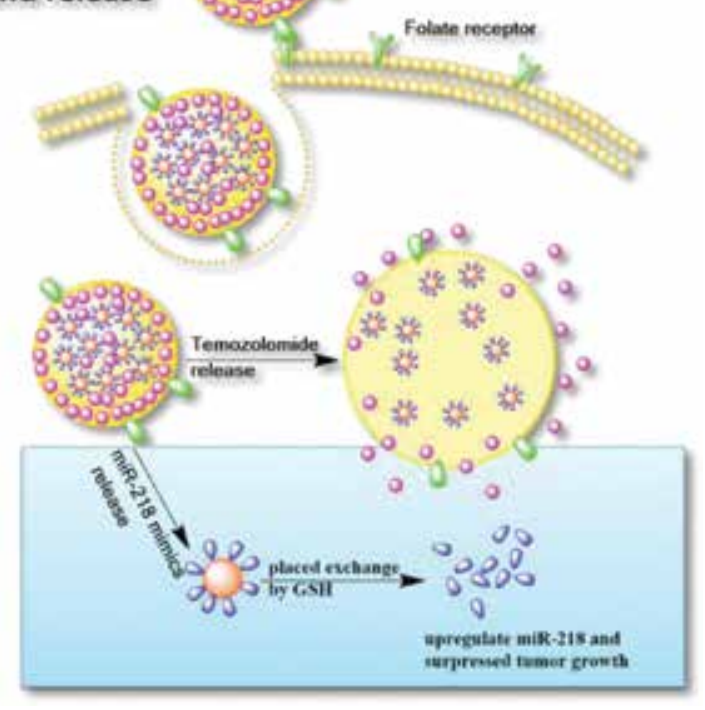

Figure 4. Schematic of drug design and drug release schedule. GSH mediated miR-218 mimics release from AuNP was emphasis by blue box [36].

Temperature is also a typical trigger at the tumor site, which could be exploited for drug delivery systems design [37, 38]. Thermo-responsive drug delivery is among the most investigated stimuli-responsive strategies. Usually, thermo-responsive nanocarriers were governed by a nonlinear sharp change with temperature, following by the release of the drug response to the temperature change. Ideally, thermo-responsive drug delivery systems should stay stable at body temperature $\left(37^{\circ} \mathrm{C}\right)$ and rapidly release the payload within a locally heated tumor $\left(40-42^{\circ} \mathrm{C}\right)$ to counteract rapid blood-passage time and washout from the tumor [38]. Poly(N-isopropyl acrylamide), PNIPAM was one of the most widely investigated thermosensitive materials, which exhibit a lower critical solution temperature. When surrounding temperature is above its LCST, the PNIPAM nanocarriers will shrink and push out the payload. For liposomes, thermos responsiveness usually arises from a phase transition of the constituent lipids and the associated conformational variations in the lipid bilayers [38, 39]. Thermoresponsive nanoparticle drug delivery systems typically present a lower critical solution temperature (LCST) at which they undergo coil-to-globule phase transitions. Thermo-sensitive liposomes usually composed of polymers with low LCST, which attached to lipid membranes due to hydrophobic interactions. The liposomes shrink to dehydrate and collapse, when the temperature achieve LCST, promoting drug release. By adjusting monomers types and ratio, polymer LCST can be tuned to different values, which could be used for controlling drug release at different environments [39].

ATP is a new member of physiological triggers to achieve "on-demand" therapeutic delivery with several merits, for example, high intracellular ATP concentration and sharp concentration 
contrast between intracellular and extracellular environment make ATP a robust trigger signal to reduce premature drug release before cellular uptake and enhance intracellular accumulation of drugs [40]. ATP-triggered drug release system provides a more sophisticated drug delivery system, which can differentiate ATP levels to facilitate the selective release of drugs. Polymeric nanocarriers functionalized with an ATP-binding aptamer-incorporated DNA motif can selectively release the intercalating doxorubicin via a conformational switch when in an ATP-rich environment [41]. However, since the ATP binding modules are basically DNA or protein, potential concerns for immunogenicity from the components need to be addressed before clinical translations.

Glucose-responsive nanoparticles were widely investigated for insulin delivery [42]. Glucose nanosensors are being incorporated to precise and accurate tracking blood glucose levels. Also, they provide the guide for glucose-responsive nanoparticles which better mimic the body's demand for insulin. Besides, glucose-sensitive self-assembly is relevant for the application of anticancer therapeutic drug delivery. Since cancer cells metabolize differently than normal cells, glucose accumulate faster in tumor site than normal tissues and circulation [43-45]. Accumulation of glucose analogue ${ }^{18}$ fluoro-2-deoxy-d-glucose $\left({ }^{18} \mathrm{FDG}\right)$ is 3.3-4.7 times greater for tumor than normal liver [46, 47]. A novel approach for glucose-triggered anticancer drug delivery from the self-assembly of neutral poly (vinyl alcohol) (PVA), and chitosan was been investigated by Satish Patil research group. This system could release glucose controllable by disintegration of layer by layer polymers. The capsules size and shape can be tuned because of physically cross-linked PVA hydrogel inside the multilayer. Because of the presence of borate in multilayer wall, the encapsulated drugs could be release programmable by different glucose concentration. The borate mediated self-assembly of PVA hydrogel and chitosan provide promising platform for intelligent anti-cancer drug delivery. The in vivo studies are under going in their laboratory [48].

Reactive oxygen species (ROS) play important roles in a variety of physiological and pathophysiological processes [49]. Moreover, many types of cancer cells exhibit high level of ROS stress [50]. An increase of $\mathrm{H}_{2} \mathrm{O}_{2}$ at cellular levels characteristic for cancer cells, which is a major component of ROS and a common marker for oxidative stress, plays a key role in carcinogenesis [51]. Thus, intracellular $\mathrm{H}_{2} \mathrm{O}_{2}$ in cancer cells was utilized as tumor site stimulus for drug delivery in cancer therapy. Synergistic release of anticancer drugs and $\mathrm{O}_{2}$ can be achieved in an $\mathrm{H}_{2} \mathrm{O}_{2}$-responsive nanocarrier incorporated with catalase. Such a system demonstrated improved therapeutic efficacy against cisplatin resistant cell lines which often appear to be in hypoxia [52]. However, the most challenging problem for engineering ROS-controlled-release systems is to improve the responsive sensitivity to ROS species, because of low concentration and very short half-lives in most cellular. Although there are increasing number of ROScontrolled release systems have been reported, development of highly sensitive nanocarriers which are specifically responsive to physiological levels of ROS are highly desired.

Till now, no optimized targeting drug delivery platform has been announced. Each has its own advantages and flaws, even for those under preclinical or clinical testing. It might be possible that the combination sequential drug delivery system design could be more effective to precise drug delivery, paving the way for a more effective personalized therapy. 


\section{Nanoparticlated formulation-based drug delivery systems}

What is such drug delivery systems composed of? Currently, many formulations of nanocarriers are utilized including lipid-based, polymeric and branched polymeric, metal-based, and mesoporous silica.

\subsection{Lipid-based nanocarriers}

The formulation of lipid-based nanomedicines against cancer has been hypothesized to improve drug localization into the tumor tissue and to increase the anticancer efficacy of conventional drugs, while minimizing their systemic adverse effects [53]. An ideal multifunctional lipid-based nanoparticle drug delivery system with targeting and triggering drug release functions should be composed of a matrix phospholipid, a destabilizing phospholipid, conjugation lipid, ligand attached, and a cell death marker. Chemotherapeutics and imaging agents were loaded in nanoparticles in aqueous phase [54]. Among various lipid-based formulations, classical examples are "liposomes," which primarily consist of phospholipids (major components of biological membranes) and have been extensively studied [55]. Prof. A.D. Bangham of the United Kingdom first published preparation of liposomes with entrapped solutes. Then, many scientists present a well-studied class of drug carriers generally characterized by the presence of a lipid bilayer that is primarily composed of amphipathic phospholipids [54].

\subsection{Polymeric and branched polymeric nanocarriers}

Polymer-based nanomedicine for improvement in efficacy of cancer therapeutics has been widely explored, including polymeric nanoparticles, polymer micelles, dendrimers, polymersomes, polyplexes, polymer-lipid hybrid systems, and polymer-drug/protein conjugates. Polymeric nanoparticles are defined by their morphology and composition. The therapeutic agent is either conjugated to the nanoparticles surface, or encapsulated and protected inside the polymeric core [56]. These polymeric nanoparticles are capable of loading wide range of drugs for a sustained or controlled release at tumor sites to provide enhanced antitumor efficacy with minimal systemic side effects. Also, these nanoparticles protect drugs from their rapid metabolism during systemic circulation and clearance by the liver, kidney, and reticuloendothelial system, which further improves drug's stability and target specificity [3, 57]. In recent years, major branch of our research was based on multifunctional poly( $\beta$-L-malic acid)based nanoconjugates [18]. This nanoconjugate with a $\mathrm{pH}$-dependent charge conversional characteristic was developed for tumor-specific drug delivery. As shown in Figure 5, nanoconjugates minimize nonspecific interactions with serum components and change the surface charge of nanoconjugates in response to the tumor acidity ( $\mathrm{pHe}$ ), leading to promoted cell internalization by the combination of electrostatic absorptive endocytosis and receptormediated endocytosis. 


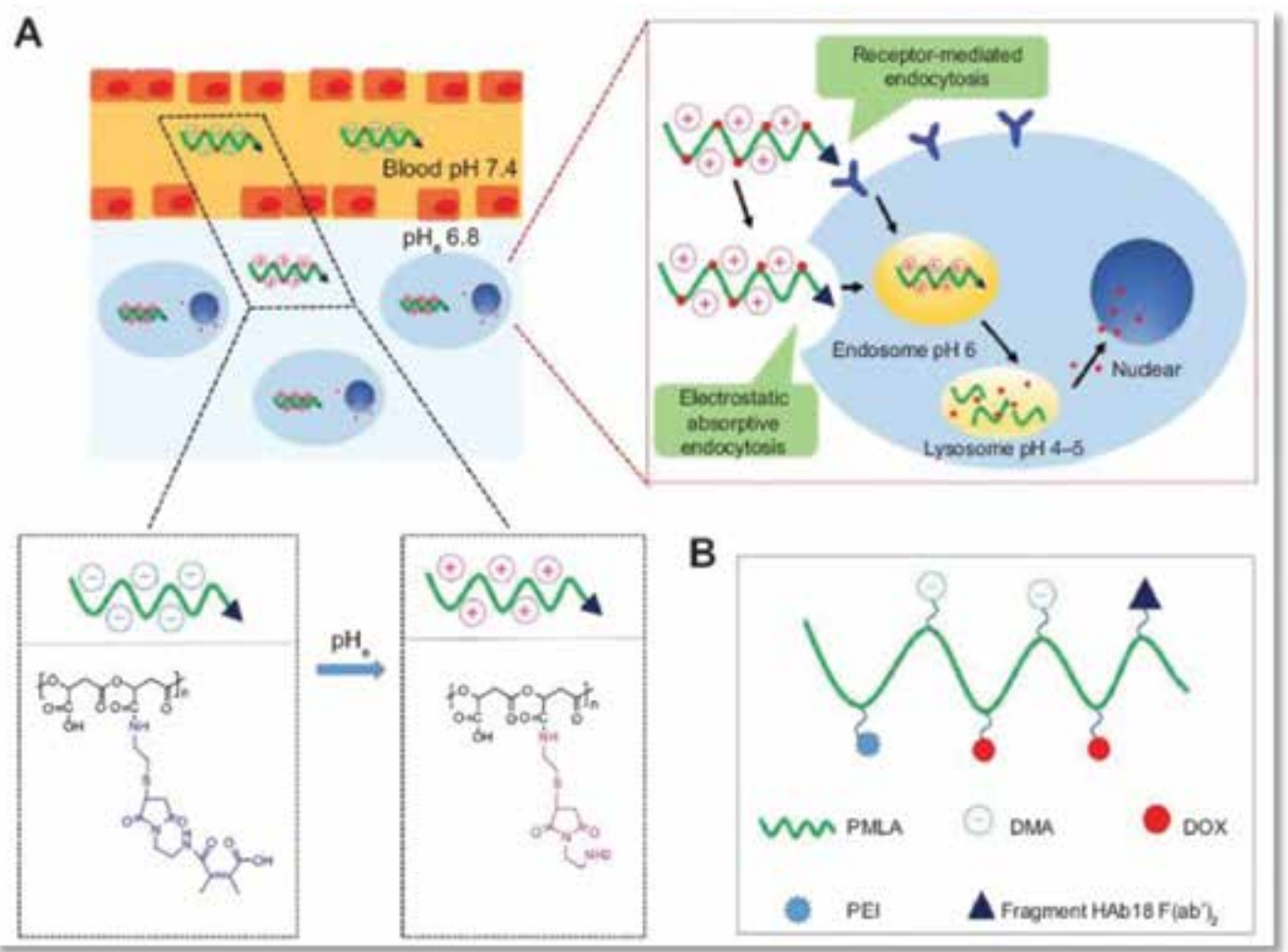

Figure 5. Schematic illustration of the stealth property and promoted tumor cell uptake of nanoconjugates (A) and DOX-loaded nanoconjugates (DOX/HDPEPM) (B). DMA, 2,3-dimethylmaleic anhydride; DOX, doxorubicin; HDPEPM, nanoconjugate formed by covalent attachment of fragment HAb18 F( $\left.\mathrm{ab}^{\prime}\right)_{2}$ and 2,3-dimethylmaleic anhydride to polyethylenimine-modified poly( $\beta$-L-malic acid); PEI, polyethylenimine; PMLA, poly( $\beta$-L-malic acid) [19].

\subsection{Metal-based nanocarriers}

Metal-based inorganic nanoparticles with monodispersity have been extensively studied for imaging using magnetic resonance and high-resolution superconducting quantum interference devices for cancer therapy [58]. Among all inorganic nanoparticles, gold nanoparticles were mostly explored for anti-tumor therapeutics delivery, due to its surface properties, strong affinity to thiol and amine functionalities and relative non-toxic nature [59]. Gold nanoparticles have been used mostly as a probe for electron microscopy and as a delivery vehicle for biomolecules. Also, super paramagnetic iron oxide nanoparticles (SPION) and gadolinium chelates are gaining interest as MRI agents [60]. Magnetic nanoparticles (MNPs) are also gaining clinical importance as MRI contrast materials, such as ferumoxides and ferumoxtran; approved by the FDA for detecting solid tumors [61]. Gadolinium-conjugated $\mathrm{TiO}_{2}$-DNA oligonucleotide nanoconjugates show prolonged intracellular retention period and T1weighted contrast enhancement in magnetic resonance images. Moreover, the increased 
retention time, Gd accumulation, and intracellular delivery may find its use in Gd neutroncapture cancer therapy [62]. Silver and platinum nanoparticles are also used for therapeutics delivery applications. Scientists at UC Santa Barbara presented a class of AgNPs that are exceptionally bright and photostable, carry peptides as model targeting ligands, can be etched rapidly and with minimal toxicity in mice, and that show tumor uptake in vivo [63]. These results illustrate how plasmonic nanoprobes based on etchable Ag cores will be a powerful tool in studies of targeted uptake and trafficking from a subcellular to tissue level. Nanoparticles built from platinum cross-linker present a novel platform for anti-tumor drug delivery. As novel cross-linker, platinum Pt (IV) diester derivative agglomerates PEG-based brush-arm star polymers (BASPs) with tunable structures was used for delivery several kinds of antitumor drug, such as doxorubicin, camptothecin, and cisplatin. The cross-linker disintegrates when reduced by glutathione, which is abundant inside cells, to release the drugs bound covalently to the star polymers. This process is well-controlled as the sizes and Pt-loading of the narrowly dispersed stars is tunable by variables such as brush length and cross-linker loading. Furthermore, in vitro and in vivo assays demonstrate an efficacy of anticancer activity and low off target toxicity [64].

\subsection{Mesoporous silica-based nanocarriers}

Multifunctional mesoporous silica nanoparticles (MSNs) are widely used as universal platform for drug delivery [65]. Highly attractive features, such as high internal surface area and pore volume, tunable pore sizes, colloidal stability, and the possibility to specifically functionalize the inner pore system and/or the external particle surface, make MSNs a promising and widely applicable platform for diverse biomedical applications including bioimaging for diagnostics [66], biosensing [67], biocatalysis [68], bone repair and scaffold engineering [69], and drug delivery [70]. For applications of multifunctional MSNs as drug delivery systems in future and further advanced in clinical trials, they should be designed with two different ways. One approach is to build up systems which could release drug response to stimuli already present in the organism, such as lower $\mathrm{pH}$ values and redox potential in endosomes (for triggered release functions). The other approach would rely on the use of external triggers (in combination with internal stimuli) to control the drug release behavior, for example, to release payloads in certain location of tissues or in certain time. Recent studies focus on the ultimate combination of diagnostic and therapeutic capabilities in the multifunctional mesoporous nanoparticles, such that the nanocarrier uses diagnostic information to control or tune its therapeutic actions [65]. Stimuli-free programmable drug release for combination chemo-therapy has been also investigated by Dr. Fan in our research group. In her previous work, she demonstrated programmed delivery of both chemotherapeutics and biodrug with tumor targeting efficacy by introducing $\mathrm{SiO}_{2}$-based self-decomposable nanoparticles. The programmable drug delivery is realized by adjusting drug loading ratios and concentration with external stimuli-free characteristics [71]. The present system provides a simple and feasible system for design targeting and combination chemotherapy with programmed drug release (Figure 6). 


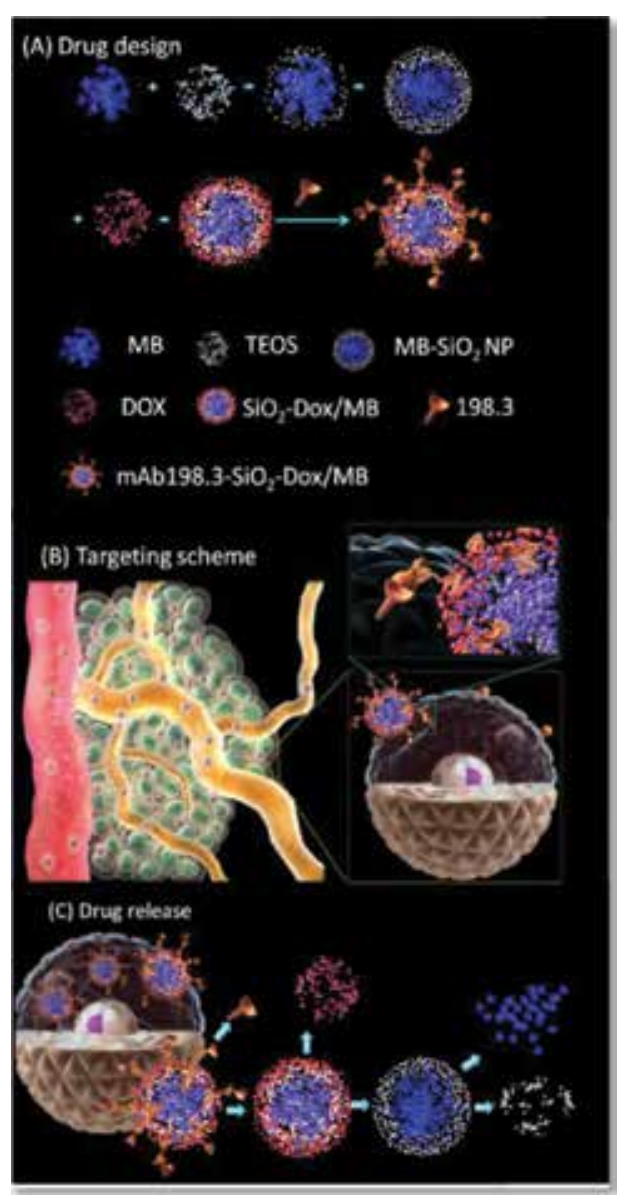

Figure 6. Schematic of the $\mathrm{SiO}_{2} \mathrm{NP}$ delivery system, its targeting scheme, and sequential drug release process. (a) Drug design of the mAb198.3-SiO - -Dox/MB NP, (b) Targeting scheme of the NP drug (c) Multi-drug release process in a sequential manner [71].

\section{Analytical techniques used for characterization of nanoparticles in vitro and in vivo}

When materials are reduced at nanoscale dimensions, they show unique properties that are different from their massive counterparts. In order to characterize nanoparticles, their particle size, size distribution, morphology, composition, surface chemistry, and reactivity are important factors that need to be defined accurately. These properties make nanomaterials a suitable carrier for unique sensing applications and, at the same time, they may also create complications during the characterization process. Choosing the right method for the characterization of nanoparticles is a challenging task since one should be aware that each technique has its own limitations. The characterization of nanoparticles is carried out through various 
techniques such as dynamic light scattering (DLS), scanning electron microscopy (SEM), transmission electron microscopy (TEM), NMR, FTIR, UV-Vis spectroscopy [72]. Techniques for cell biology, such as TEM, confocal laser scanning microscopy (CLSM), and flow cytometry, were employed to evaluate target ability of nanodrugs in vitro. In vivo imaging system and drug biodistribution were used to assess the in vivo behavior and efficacy of nanodrugs.

\subsection{Dynamic light scattering (DLS) analysis}

The size of nanoparticles is one of the key parameters that influence the interaction between nanoparticles and cells, which influenced cellular uptake [73, 74]. DLS is the most suitable technique to determine the particle size of nanoparticles (Figure 7).

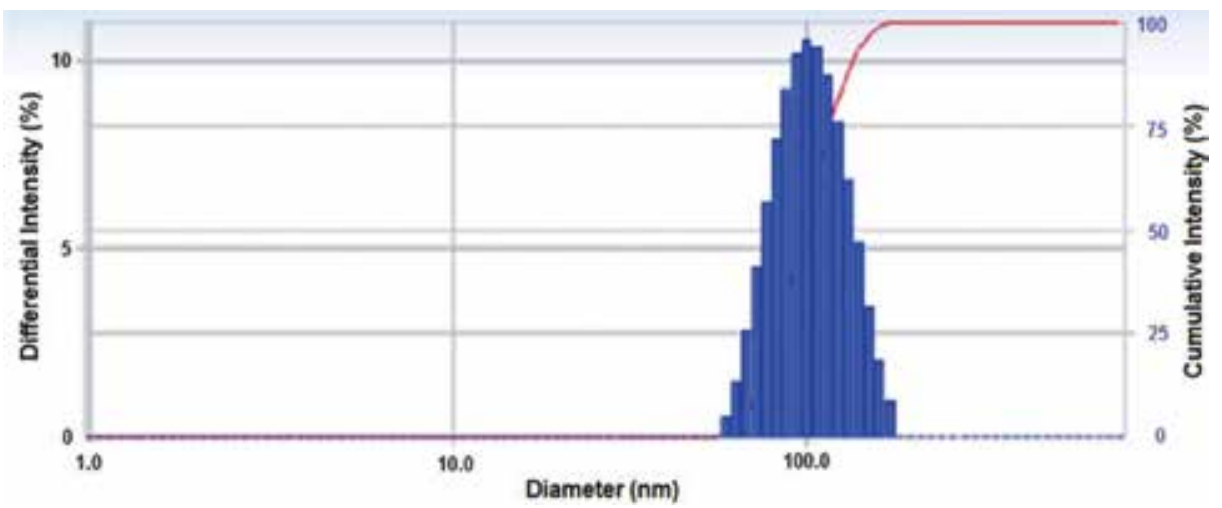

Figure 7. Particle size and size distribution of nanoparticles.

DLS is a technique in physics that can be used to determine the size distribution profile of small particles in suspension or polymers in solution, by measuring the random changes in the intensity of light scattered based on dynamic Brownian motion of the suspended particle. This technique is also called photon correlation spectroscopy (PCS) and quasi-elastic light scattering (QELS). The latter terms are more common in older literature. Typical applications are emulsions, micelles, polymers, proteins, nanoparticles or colloids. In general, the technique is best used for submicron particles and can be used to measure particle with sizes less than a nanometer. In this size regime (microns to nanometers) and for the size measurement (but not thermodynamics), the distinction between a molecule (such as a protein or macromolecule) and a particle and even a second liquid phase (such as in an emulsion) becomes blurred.

There are several advantages associated with DLS: simplicity; sensitivity and selectivity to NPs; short time of measurement; and the fact that calibration is not needed. Therefore, this technique is increasingly used for nanoparticle characterization in various science and industry fields $[75,76]$. However, some problems are encountered when measuring samples with larger size distributions or multimodal distributions [77]. If the measured colloid is monodispersed, the mean diameter of the nanoparticles can be determined using the DLS technique. For polydispersed colloids, there is a risk during the DLS measurement, as small 
particles can be screened by bigger particles, since bigger particles have more scattering property .

Some DLS instrument can measure not only particle size, but also Zeta potential at the same time [78]. Zeta potential is the surface charge of nanoparticles in solution (colloids). Nanoparticles have a surface charge that attracts a thin layer of ions of opposite charge to the nanoparticles surface. This double layer of ions travels with the nanoparticle as it diffuses throughout the solution. The electric potential at the boundary of the double layer is known as the Zeta potential of the particles and has values that typically range from $+100 \mathrm{mV}$ to $-100 \mathrm{mV}$. Zeta potential is an important tool for understanding the state of the nanoparticle surface and predicting the long-term stability of the nanoparticle (Figure 8).
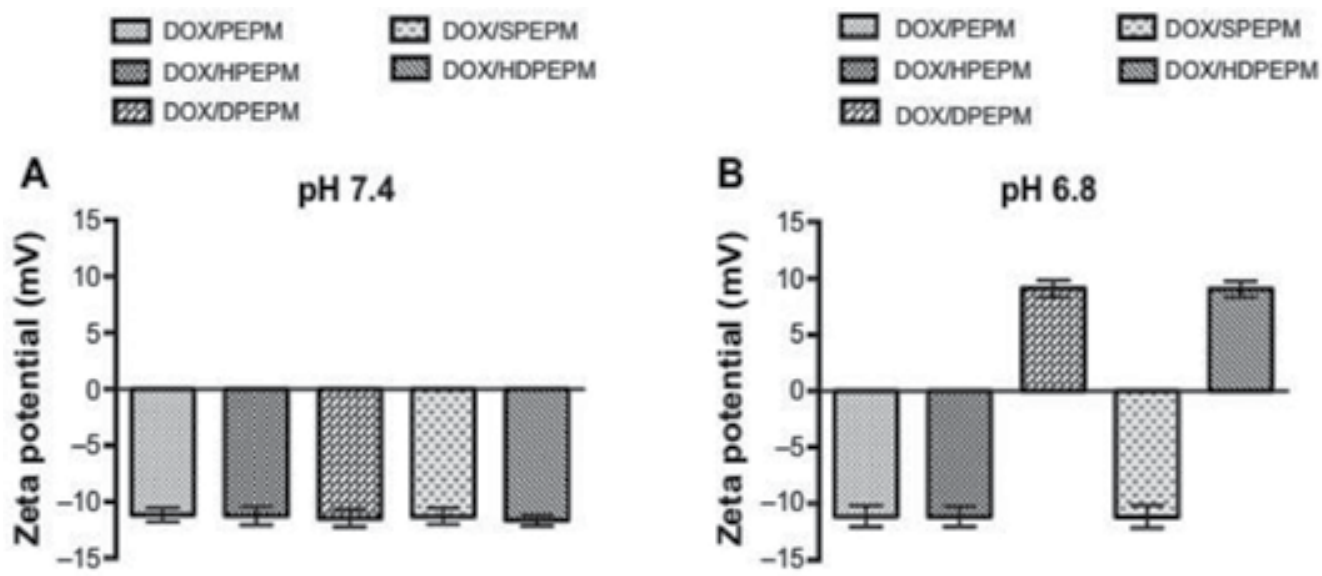

Figure 8. Zeta potential of different DOX-loaded nanoconjugates at (A) $\mathrm{pH} 7.4$ and $(\mathrm{B}) \mathrm{pH} 6.8(\mathrm{n}=5)$. At $\mathrm{pH} 7.4$, no charge-conversional behaviors were observed. When the $\mathrm{pH}$ was decreased from 7.4 to 6.8, both DOX/DPEPM and DOX/HDPEPM nanoconjugates showed a significant charge conversion [19].

\subsection{Transmission electron microscope (TEM) and scanning electronic microscopy (SEM)}

Particle morphology is another important parameter for the characterization of nanoparticles, and this is achieved with the help of microscopic techniques such as SEM and TEM. Both techniques produce a resolution that is a thousand times greater than the optical diffraction limit. SEM uses a beam of high-energy electrons to produce a variety of signals that contain information about the sample's surface composition, topography, and other properties such as electrical conductivity. We can analyze the sample at various times because $X$-rays generated by SEM do not lead to a loss of volume of the sample. However, electron microscopy creates a risk of radiation damage that is caused by the electron beam, which leads to the generation of free radicals. The diffusion of free radicals and the loss of mass may cause physical damage to the sample [78]. Also, TEM suffers from the limitations of poor contrast, especially in the event of peptide/protein nanoparticles and their conjugates. Besides particle morphology, TEM and SEM could also be used to study the physical size of nanoparticles (Figure 9). 
However, there are some disadvantages associated with TEM and SEM: time consuming, high operator fatigue, few particles examined.

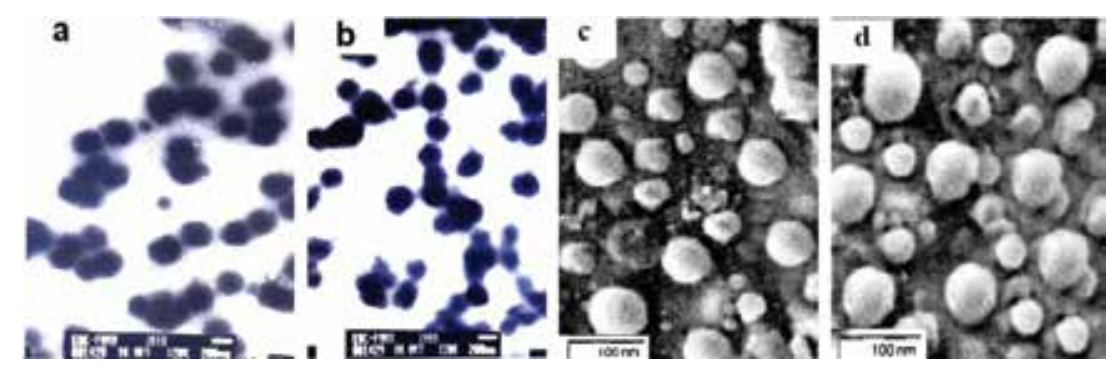

Figure 9. TEM and SEM micrographs of blank and drug-loaded nanoparticles (a) TEM of blank nanoparticles; (b) TEM of drug-loaded nanoparticles; (c) SEM of blank nanoparticles; (d) SEM of drug-loaded nanoparticles [79].

\subsection{NMR, FTIR, and UV-Vis spectroscopy}

NMR, FTIR, and UV-Vis spectroscopies are primary methods for determining the structure of compounds. They are also used in analyzing the structure of nanoparticles, especially to confirm the modification of polymer carriers. These are simply done and rapid. They can be combined to give overlapping information. NMR spectroscopy is one of the most nondestructive techniques in elucidating molecular structure as well as understanding the molecular dynamics of organic, organometallic, inorganic, polymeric, and biological molecules (Figure 10). It can be also used in nanoparticle size determination and nanoparticle surface study [80, 81]. IR spectra can be used to provide information on the functional groups as well as the structure of a molecule as a whole. UV-Vis spectra have broad features that could provide only limited information of structure but very useful for quantitative measurements.

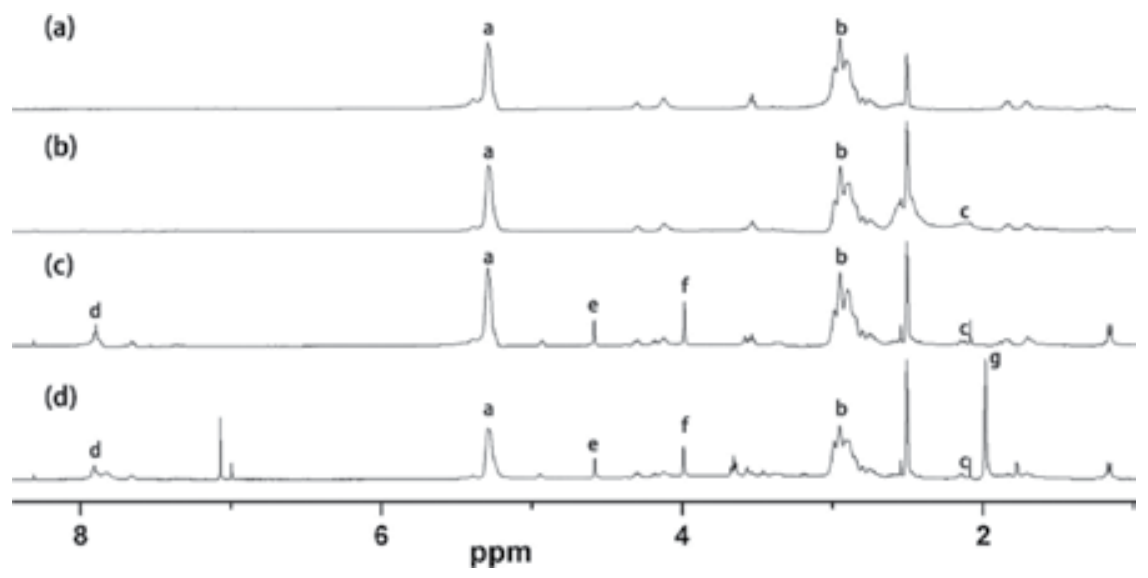

Figure 10. ${ }^{1} \mathrm{H}$ NMR spectra of (a) PMLA, (b) PEI-PMLA (PEPM), (c) DOX/PEPM, and (d)DOX/DPEPM. DMSO was used as the solvent [19]. 
The ability to enter target cell efficiently is a key character of nanoparticles. Techniques for cell biology, such as confocal microscopy, flow cytometry, were employed to evaluate target ability of nanodrugs in vitro.

\subsection{Confocal laser scanning microscopy (CLSM)}

CLSM is a technique for obtaining high-resolution optical images with depth selectivity. The key feature of CLSM is its ability to acquire the in-focus images from selected depths, a process known as optical sectioning. It could be used to observe the cellular uptake of fluorescence labeled nanoparticles, as well as nanoparticles-cell interaction (Figure 11).

\section{AuCOOH(Cy5)_isotype (negative control) incubation for $4 \mathrm{~h}$}

AuCOOH(Cy5)_mAb198.3 incubation for $15 \mathrm{~min}$

\section{AuCOOH(Cy5)_mAb198.3} incubation for $30 \mathrm{~min}$

\section{AuCOOH(Cy5)_mAb198.3 incubation for $\mathbf{4 h}$}

\section{DAPI}
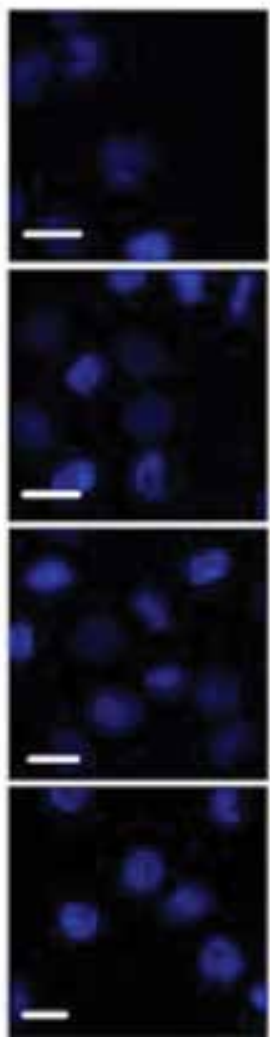

Cy5
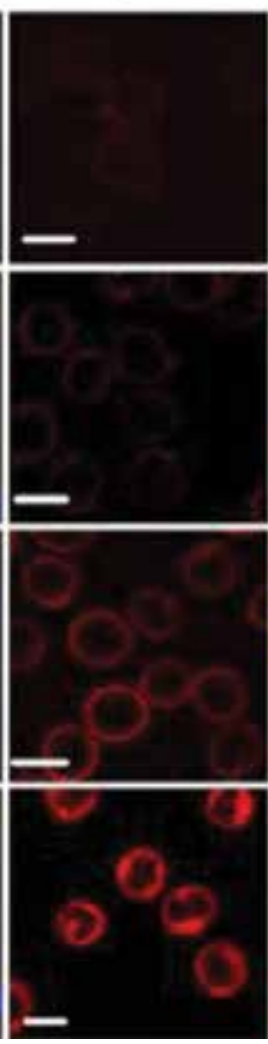

Merge
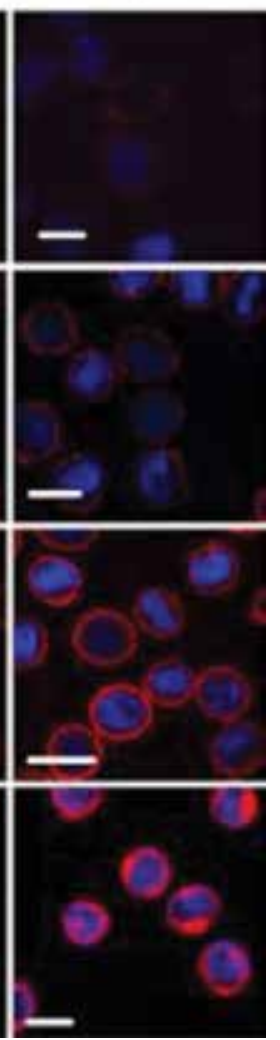

Figure 11. Confocal images of Colo 205 cells incubated with $\mathrm{AuCOOH}(\mathrm{Cy} 5)$ _isotype (negative control) and Au$\mathrm{COOH}(\mathrm{Cy} 5) \_\mathrm{mAb} 198.3$ and nucleus stained with DAPI. Incubated time: $15 \mathrm{~min}, 30 \mathrm{~min}$ and $4 \mathrm{~h}$. (Blue fluorescence is associated with DAPI, and red fluorescence is associated with Cy5). Scale bar at $20 \mu \mathrm{m}$ [36].

\subsection{Flow cytometry}

Flow cytometry is a laser-based, biophysical technology employed in cell counting, cell sorting, biomarker detection, and protein engineering, by suspending cells in a stream of fluid and 
passing them by an electronic detection apparatus. It is extensively used in research for the cell apoptosis and fluorescence quantitative analysis of nanoparticles to evaluate its targeting efficacy (Figure 12).

\section{pH 6.8}
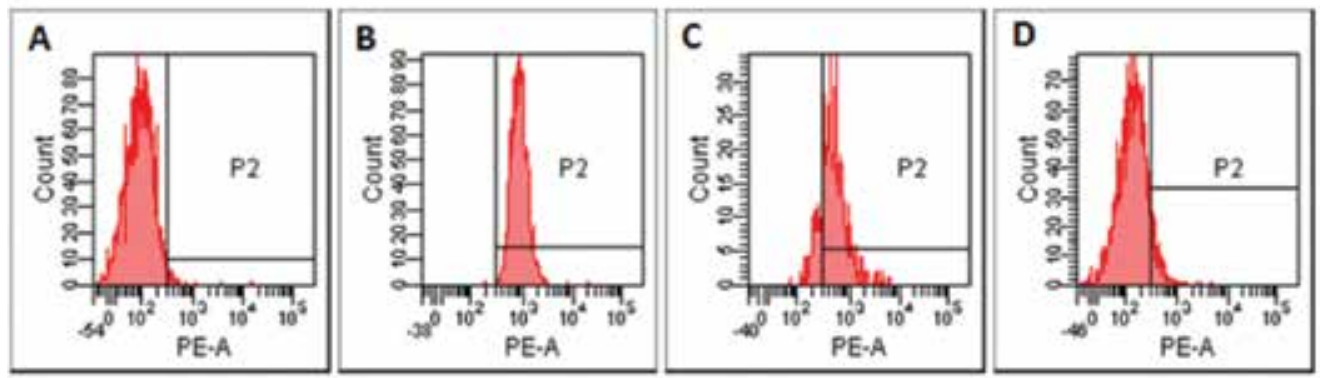

\section{pH 7.4}
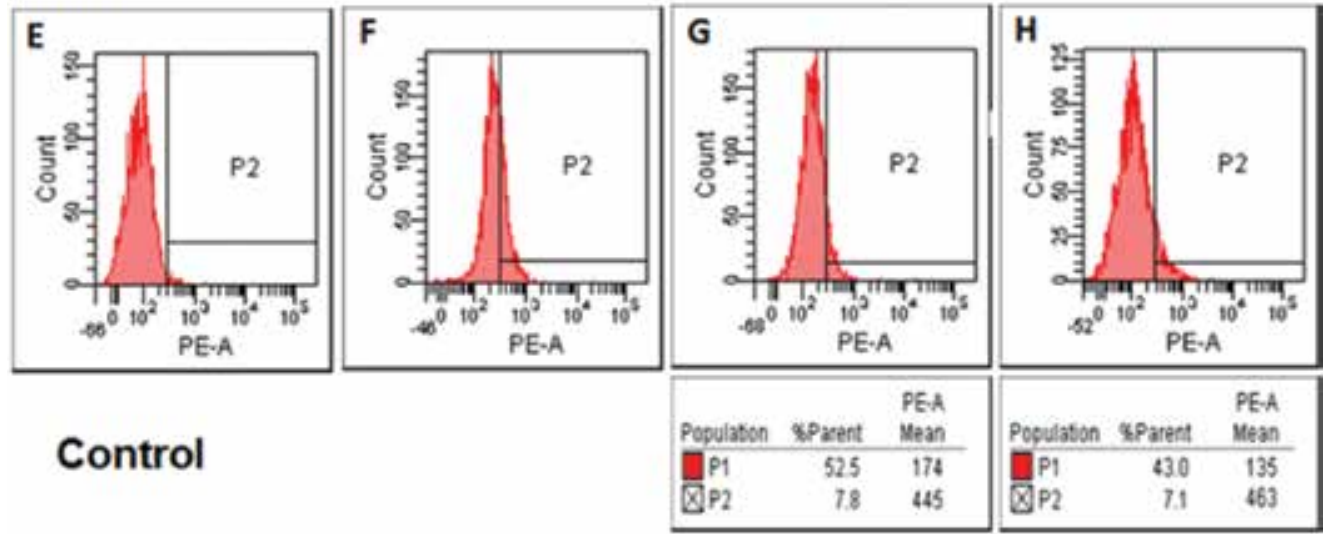

Figure 12. FACS analysis of A2780/DoxR cells incubated for $1 \mathrm{~h}$ at $37^{\circ} \mathrm{C}$ with untreated cell as control (A, E), LHRHPEG-PHIS-Dox/Dox-TAT (B, F), LHRH-PEG-PHIS-Dox/Dox (C, G) and LHRH-PEG-PHIS-Dox (D, H) at pH 7.4 or pH 6.8 , respectively [18].

\subsection{In vivo imaging system}

The ability of nanoparticles to achieve high, local concentrations of drugs at a target site provides the opportunity for improved system performance and patient outcomes along with reduced systemic dosing. Current technologies for tumor imaging, such as in vivo imaging system, are able to yield high-resolution images for the assessment of nanoparticles uptake in tumors at the microscopic level; a microscopic visual representation of a biological component inside the body [82]. The imaging procedure often utilizes a variety of diagnostic tools to provide insight regarding disease states, molecular characterization, and biological processes (Figure 13). 

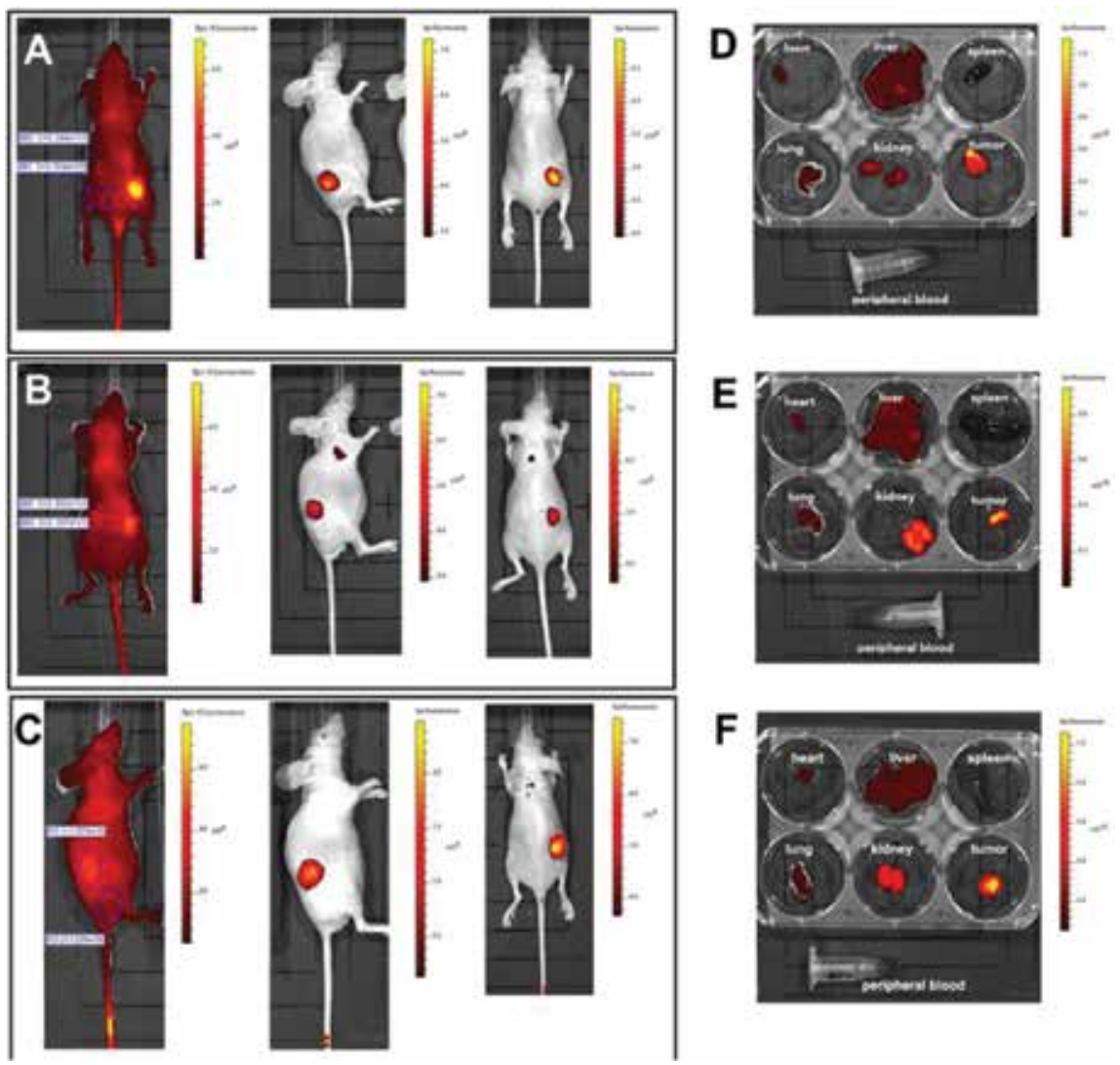

Figure 13. In vivo imaging of Colo 205 tumor bearing mice. Fluorescent signal captured by IVIS Lumina Imaging System in tumor bearing mice after injection with $\mathrm{AuCOOH}(\mathrm{Cy} 5)_{-}$mAb198.3 (a), $\mathrm{AuCOOH}(\mathrm{Cy} 5)_{-}$isotype (b), and mAb198.3_Cy5 (c) for 24 h. Luminescent image of resected organs from Colo 205 tumor-bearing mouse injected with $\mathrm{AuCOOH}(\mathrm{Cy5}) \_\mathrm{mAb} 198.3$ (d), $\mathrm{AuCOOH}(\mathrm{Cy5}) \_$isotype (e), and mAb198.3_Cy5 (f) for $24 \mathrm{~h}$ [36].

\subsection{Drug biodistribution analysis}

Another method to assess the in vivo behavior and efficacy of nanodrugs is drug biodistribution analysis. This is a method of tracking where drugs of interest travel in an experimental animal or human subject by the determination of drug concentration in targeted site and other organs.

\section{Disadvantages and challenges of nanodrug}

Nanodrug since its emergence has proved to be promising novel drug delivery system. In recent years, great progress was achieved in making drugs owning the characteristics of targeted and controlled release via nanotechnologies. However, there are some challenges in the use of large size materials in drug delivery. Some of these challenges are poor targeting and therapeutic effects, sustained and targeted delivery to site of action, poor bioavailability, 
generalized side effects, in vivo stability, intestinal absorption, and plasma fluctuations of drugs [83]. Taking the active targeting strategy as an example, it is not always as effective as expected. The main mechanism behind active targeting is the recognition of the ligand by its target substrate. But because of the heterogeneity of tumor cells, receptors on the surface of tumor cells are different from cell to cell. Therefore, the interaction between cell receptors and ligands linked to nanoparticles becomes unreliable, which the nanoparticles was relied on to enter into the cell. This results in poor targeting and therapeutic effects in some cases [84]. Besides, distribution through the tumor is severely limited by its relatively large size which slows diffusion and may become trapped in the ECM. Other obstacles with nanocarriers that must be concerned include complicated synthesis, in vivo aggregation and recognition by the reticuloendothelial system leading to high clearance. This is further complicated when the therapeutic is covalently attached to the drug carrier as in the case of many polymers. Finally, most studies are at the basic research stage at present. Since it was unknown about environmental influence and genetic effect of novel nanomaterials, much works and a long process for acceptance by public were needed for more nanodrugs to be used in clinic.

To reach the promise of nanodrugs, it is necessary to take a step back and look at the problems facing drug delivery as a whole rather than designing around only one or two obstacles. Incremental designs may not be sufficient to accomplish the task of treating cancer effectively. Instead, a revolution in concept is needed. Nanodrug delivery system with simple synthesis routes and high targeting/therapeutic efficacy may point the way out.

So far, there are so many publications but so few nanodrugs in cancer therapy [85]. The uncertainty and limitation of nanodrugs in pharmacology, toxicology, immunology, largescale manufacturing, and regulatory issues make it become an important research field in nanoparticle-based tumor targeting delivery system. And how we can overcome these difficulties, it is a long way to go.

\section{Acknowledgements}

This work was supported by the National Nature Science Foundation of China (grants number 81271687, 81571786, and 81373948). Reproduced from Ref. 14, 18, 19, 36, 71, and 76 with permission from Elsevier, Royal Society of Chemistry, Dove Medical Press Ltd. and BioMed Central, respectively.

\section{Author details}

Hong $\mathrm{Wu}^{*}$, Tiehong Yang and Li Fan

*Address all correspondence to: wuhong@fmmu.edu.cn

School of Pharmacy, Fourth Military Medical University, Xi'an, China 


\section{References}

[1] Bamrungsap S, Zhao Z, Chen T, Wang L, Li C, Fu T, Tan W. Nanotechnology in therapeutics: a focus on nanoparticles as a drug delivery system. Nanomedicine, 2012, 7: 1253-71.

[2] Matsumura Y, Maeda H. A new concept for macromolecular therapeutics in cancer chemotherapy: mechanism of tumoritropic accumulation of proteins and the antitumor agent smancs. Cancer Res, 1986, 46: 6387-92.

[3] Sinha R, Kim GJ, Nie S, Shin DM. Nanotechnology in cancer therapeutics: bioconjugated nanoparticles for drug delivery. Mol Cancer Ther, 2006, 5: 1909-17.

[4] Smith AM, Duan H, Mohs AM, Nie S. Bioconjugated quantum dots for in vivo molecular and cellular imaging. Adv Drug Deliver Rev, 2008, 60: 1226-40.

[5] Srikanth M, Kessler JA. Nanotechnology-novel therapeutics for CNS disorders. Nat Rev Neurol, 2012, 8: 307-18.

[6] Wicki A, Witzigmann D, Balasubramanian V, Huwyler J. Nanomedicine in cancer therapy: challenges, opportunities, and clinical applications. J Control Release, 2015, 200: 138-57.

[7] Maeda H. Tumor-selective delivery of macromolecular drugs via the EPR effect: background and future prospects. Bioconjugate Chem, 2010, 21: 797-802.

[8] Torchilin V. Tumor delivery of macromolecular drugs based on the EPR effect. Adv Drug Deliver Rev, 2011, 63: 131-5.

[9] Tanaka T, Shiramoto S, Miyashita M, Fujishima Y, Kaneo Y. Tumor targeting based on the effect of enhanced permeability and retention (EPR) and the mechanism of receptormediated endocytosis (RME). Int J Pharm, 2004, 277: 39-61.

[10] Jain RK. Barriers to drug delivery in solid tumors. Sci Am, 1994, 271: 58-65.

[11] Sriraman SK, Aryasomayajula B, Torchilin VP. Barriers to drug delivery in solid tumors. Tissue Barriers, 2014, 2: e29528.

[12] Jain RK, Stylianopoulos T. Delivering nanomedicine to solid tumors. Nat Rev Clin Oncol, 2010, 7: 653-64.

[13] Gaumet M, Vargas A, Gurny R, Delie F. Nanoparticles for drug delivery: the need for precision in reporting particle size parameters. Eur J Pharm Biopharm, 2008, 69: 1-9.

[14] Fan L, Zhang Y, Wang F, Yang Q, Tan J, Renata G, Wu H, Song C, Jin B. Multifunctional all-in-one drug delivery systems for tumor targeting and sequential release of three different anti-tumor drugs. Biomaterials, 2016, 76: 399-407.

[15] Jhaveri A, Torchilin V. Intracellular delivery of nanocarriers and targeting to subcellular organelles. Expert Opin Drug Del, 2016, 13: 49-70. 
[16] Wu Q, Wu L, Wang Y, Zhu Z, Song Y, Tan Y, Wang XF, Li J, Kang D, Yang CJ. Evolution of DNA aptamers for malignant brain tumor gliosarcoma cell recognition and clinical tissue imaging. Biosens Bioelectron, 2016, 80, 1-8.

[17] Fan L, Li F, Zhang H, Wang Y, Cheng C, Li X, Gu CH, Yang Q, Wu H, Zhang S. Codelivery of PDTC and doxorubicin by multifunctional micellar nanoparticles to achieve active targeted drug delivery and overcome multidrug resistance. Biomaterials, 2010, 31: 5634-42.

[18] Yang T, Li F, Zhang H, Fan L, Qiao Y, Tan G, Zhang H, Wu H. Multifunctional pHsensitive micelles for tumor-specific uptake and cellular delivery. Polym Chem, 2015, 6(8): 1373-82.

[19] Zhou Q, Yang T, Qiao Y, Guo S, Zhu L, Wu H. Preparation of poly(beta-L-malic acid)based charge-conversional nanoconjugates for tumor-specific uptake and cellular delivery. Int J Nanomed, 2015, 10: 1941-52.

[20] Farokhzad OC, Langer R. Impact of nanotechnology on drug delivery. ACS Nano, 2009, 3: 16-20.

[21] Crommelin DJ, Park K, Florence A. Pharmaceutical nanotechnology: unmet needs in drug delivery. J Contro Release, 2010, 141: 263-4.

[22] Yang L, Webster TJ. Nanotechnology controlled drug delivery for treating bone diseases. Expert Opin Drug Del, 2009, 6: 851-64.

[23] Shirshahi V, Shamsipour F, Zarnani AH, Verdi J, Saber R. Active targeting of HER2positive breast cancer cells by Herceptin-functionalized organically modified silica nanoparticles. Cancer Nanotechnol, 2013, 4: 27-37.

[24] Master AM, Sen Gupta A. EGF receptor-targeted nanocarriers for enhanced cancer treatment. Nanomed, 2012, 7: 1895-906.

[25] Deshpande PP, Biswas S, Torchilin VP. Current trends in the use of liposomes for tumor targeting. Nanomed, 2013, 8: 1509-28.

[26] Huang BN, Abraham WD, Zheng YR, Lopez SCB, Luo SS, Irvine DJ. Active targeting of chemotherapy to disseminated tumors using nanoparticle-carrying T cells. Sci Transl Med, 2015, 7(291): 291ra94.

[27] Zhang Y, Hong H, Cai W. Tumor-targeted drug delivery with aptamers. Curr Med Chem, 2011, 18: 4185-94.

[28] Zwicke GL, Mansoori GA, Jeffery CJ. Utilizing the folate receptor for active targeting of cancer nanotherapeutics. Nano Rev, 2012, 3: 18496-http://dx.doi.org/10.3402/ nano.v3i0.18496.

[29] Kapse-Mistry S, Govender T, Srivastava R, Yergeri M. Nanodrug delivery in reversing multidrug resistance in cancer cells. Front Pharmaco, 2014, 5: 159. 
[30] Zhu CL, Wang XW, Lin ZZ, Xie ZH, Wang XR. Cell microenvironment stimuliresponsive controlled-release delivery systems based on mesoporous silica nanoparticles. J Food Drug Anal, 2014, 22: 18-28.

[31] Lee ES, Gao Z, Bae YH. Recent progress in tumor pH targeting nanotechnology. J Control Release, 2008, 132: 164-70.

[32] Yang ZZ, Li JQ, Wang ZZ, Dong DW, Qi XR. Tumor-targeting dual peptides-modified cationic liposomes for delivery of siRNA and docetaxel to gliomas. Biomaterials, 2014, 35: 5226-39.

[33] Zhang W, Dong D, Li P, Wang D, Mu H, Niu H, Duan J. Novel pH-sensitive polysialic acid based polymeric micelles for triggered intracellular release of hydrophobic drug. Carbohyd Polym, 2016, 139: 75-81.

[34] Cheng R, Feng F, Meng F, Deng C, Feijen J, Zhong Z. Glutathione-responsive nanovehicles as a promising platform for targeted intracellular drug and gene delivery. $J$ Control Release, 2011, 152: 2-12.

[35] Hong R, Han G, Fernandez JM, Kim BJ, Forbes NS, Rotello VM. Glutathione-mediated delivery and release using monolayer protected nanoparticle carriers. J Am Chem Soc, 2006, 128: 1078-9.

[36] Fan L, Yang Q, Tan J, Qiao Y, Wang Q, He J, Wu H, Zhang Y. Dual loading miR-218 mimics and Temozolomide using AuCOOH@FA-CS drug delivery system: promising targeted anti-tumor drug delivery system with sequential release functions. J Exp Clin Canc Res: CR 2015, 34: 106.

[37] Shao PY, Wang BC, Wang YZ, Li J, Zhang YQ. The application of thermosensitive nanocarriers in controlled drug delivery. J Nanomater, 2012, 23(50): 505706-18.

[38] Mura S, Nicolas J, Couvreur P. Stimuli-responsive nanocarriers for drug delivery. Nat Mater, 2013, 12: 991-1003.

[39] Ta T, Porter TM. Thermosensitive liposomes for localized delivery and triggered release of chemotherapy. J Control Release, 2013, 169: 112-25.

[40] Sun W, Gu Z. ATP-responsive drug delivery systems. Expert Opin Drug Del, 2016, 1-4.

[41] Mo R, Jiang T, DiSanto R, Tai W, Gu Z. ATP-triggered anticancer drug delivery. Nat Commun, 2014, 5: 3364.

[42] Veiseh O, Tang BC, Whitehead KA, Anderson DG, Langer R. Managing diabetes with nanomedicine: challenges and opportunities. Nat Rev Drug Discov, 2015, 14: 45-57.

[43] Hahn T, Barth S, Hofmann W, Reich O, Lang I, Desoye G. Hyperglycemia regulates the glucose-transport system of clonal choriocarcinoma cells in vitro. A potential molecular mechanism contributing to the adjunct effect of glucose in tumor therapy. Int J Cancer, 1998, 78: 353-60. 
[44] Okumura M, Yamamoto M, Sakuma H, Kojima T, Maruyama T, Jamali M, Cooper DR, Yasuda K. Leptin and high glucose stimulate cell proliferation in MCF-7 human breast cancer cells: reciprocal involvement of PKC-alpha and PPAR expression. Biochim Biophys Acta, 2002, 1592(2): 107-16.

[45] Ganapathy V, Thangaraju M, Prasad PD. Nutrient transporters in cancer: relevance to Warburg hypothesis and beyond. Pharmacol Therapeut, 2009, 121: 29-40.

[46] Nolop KB, Rhodes CG, Brudin LH, Beaney RP, Krausz T, Jones T, Hughes JM. Glucose utilization in vivo by human pulmonary neoplasms. Cancer, 1987, 60: 2682-9.

[47] Yonekura Y, Benua RS, Brill AB, Som P, Yeh SDJ, Kemeny NE, Fowler JS, Acgregor RR, Stamm R, Christman DR, Wolf AP. Increased accumulation of 2-deoxy-2-[F-18]fluoroD-glucose in liver metastases from colon-carcinoma. J Nucl Med, 1982, 23: 1133-7.

[48] Manna U, Patil S. Glucose-triggered drug delivery from borate mediated layer-by-layer self-assembly. ACS Appl Mater Inter, 2010, 2: 1521-7.

[49] Li JJ, Han Y, Chen QX, Shi HD, Rehman SU, Siddiq M, Ge ZS, Liu SY. Dual endogenous stimuli-responsive polyplex micelles as smart two-step delivery nanocarriers for deep tumor tissue penetration and combating drug resistance of cisplatin. J Mater Chem B, 2014, 2: 1813-1824.

[50] Trachootham D, Alexandre J, Huang P. Targeting cancer cells by ROS-mediated mechanisms: a radical therapeutic approach? Nat Rev Drug Dis, 2009, 8: 579-91.

[51] Lopez-Lazaro M. Dual role of hydrogen peroxide in cancer: possible relevance to cancer chemoprevention and therapy. Cancer Lett, 2007, 252: 1-8.

[52] Chen H, He W, Guo Z. An $\mathrm{H}(2) \mathrm{O}(2)$-responsive nanocarrier for dual-release of platinum anticancer drugs and $\mathrm{O}(2)$ : controlled release and enhanced cytotoxicity against cisplatin resistant cancer cells. Chem Commun, 2014, 50: 9714-7.

[53] Arias JL, Clares B, Morales ME, Gallardo V, Ruiz MA. Lipid-based drug delivery systems for cancer treatment. Currt Drug Targets, 2011, 12, 1151-65.

[54] Puri A, Loomis K, Smith B, Lee JH, Yavlovich A, Heldman E, Blumenthal R. Lipidbased nanoparticles as pharmaceutical drug carriers: from concepts to clinic. Crit Rev Ther Drug, 2009, 26: 523-80.

[55] Fenske DB, Chonn A, Cullis PR. Liposomal nanomedicines: an emerging field. Toxicol Pathol, 2008, 36: 21-9.

[56] Prabhu RH, Patravale VB, Joshi MD. Polymeric nanoparticles for targeted treatment in oncology: current insights. Int J Nanomed, 2015, 10: 1001-18.

[57] Chan JM, Valencia PM, Zhang L, Langer R, Farokhzad OC. Polymeric nanoparticles for drug delivery. Meth Mol Biology, 2010, 624, 163-75. 
[58] Alexis F, Pridgen EM, Langer R, Farokhzad OC. Nanoparticle technologies for cancer therapy. Handbook of Experimental Pharmacology, Rosenthal, W., Ed. Springer Science +Business Media: Heidelberg, 2010, 55-86.

[59] Patra CR, Bhattacharya R, Mukhopadhyay D, Mukherjee P. Application of gold nanoparticles for targeted therapy in cancer. J Biomed Nanotechnol, 2008, 4: 99-132.

[60] Liu Y, Miyoshi H, Nakamura M. Nanomedicine for drug delivery and imaging: a promising avenue for cancer therapy and diagnosis using targeted functional nanoparticles. Int J Cancer, 2007, 120: 2527-37.

[61] Shubayev VI, Pisanic TR. 2nd; Jin S. Magnetic nanoparticles for theragnostics. Adv Drug Del Rev, 2009, 61: 467-77.

[62] Paunesku T, Ke T, Dharmakumar R, Mascheri N, Wu AG, Lai B, Vogt S, Maser J, Thurn K, Szolc-Kowalska B, Larson A, Bergan RC, Omary R, Li DB, Lu ZR, Woloschak GE. Gadolinium-conjugated TiO2-DNA oligonucleotide nanoconjugates show prolonged intracellular retention period and T1-weighted contrast enhancement in magnetic resonance images. Nanomed-Nanotechnol, 2008, 4: 201-207.

[63] Braun GB, Friman T, Pang HB, Pallaoro A, Hurtado de Mendoza T, Willmore AM, Kotamraju VR, Mann AP, She ZG, Sugahara KN, Reich NO, Teesalu T, Ruoslahti E. Etchable plasmonic nanoparticle probes to image and quantify cellular internalization. Nature Mater, 2014, 13: 904-11.

[64] Liao L, Liu J, Dreaden EC, Morton SW, Shopsowitz KE, Hammond PT, Johnson JA. A convergent synthetic platform for single-nanoparticle combination cancer therapy: ratiometric loading and controlled release of cisplatin, doxorubicin, and camptothecin. J Am Chem Soc, 2014, 136: 5896-9.

[65] Argyo C, Weiss V, Brauchle C, Bein T. Multifunctional mesoporous silica nanoparticles as a universal platform for drug delivery. Chem Mater, 2014, 26: 435-451.

[66] Lee JE, Lee N, Kim T, Kim J, Hyeon T. Multifunctional mesoporous silica nanocomposite nanoparticles for theranostic applications. Account of Chem Res, 2011, 44: 893-902.

[67] Liu JL, Li CY, Li FY. Fluorescence turn-on chemodosimeter-functionalized mesoporous silica nanoparticles and their application in cell imaging. J Mater Chem, 2011, 21: 717581.

[68] Ariga K, Ji Q, Mori T, Naito M, Yamauchi Y, Abe H, Hill JP. Enzyme nanoarchitectonics: organization and device application. Chem Soc Rev, 2013, 42: 6322-45.

[69] Salinas AJ, Esbrit P, Vallet-Regi M. A tissue engineering approach based on the use of bioceramics for bone repair. Biomater Sci-Uk, 2013, 1: 40-51.

[70] Li Z, Barnes JC, Bosoy A, Stoddart JF, Zink JI. Mesoporous silica nanoparticles in biomedical applications. Chem Soc Rev, 2012, 41: 2590-605. 
[71] Fan L, Jin B, Zhang S, Song C, Li Q. Stimuli-free programmable drug release for combination chemo-therapy. Nanoscale, 2016.

[72] Zaman M, Ahmad E, Qadeer A, Rabbani G, Khan RH. Nanoparticles in relation to peptide and protein aggregation. Int J Nanomedicine, 2014, 9: 899-912.

[73] Desai MP, Labhasetwar V, Walter E, Levy RJ, Amidon GL. The mechanism of uptake of biodegradable microparticles in Caco-2 cells is size dependent. Pharm Res, 1997, 14(11): 1568-1573.

[74] Zauner W, Farrow NA, Haines AM. In vitro uptake of polystyrene microspheres: effect of particle size, cell line and cell density. J Control Release, 2001;71(1): 39-51.

[75] Brar SK, Verma M. Measurement of nanoparticles by light-scattering techniques. $\operatorname{Tr} A C$ - Trend Anal Chem, 2011, 30(1): 4-17.

[76] Khlebtsov BN, Khlebtsov NG. On the measurement of gold nanoparticle sizes by the dynamic light scattering method. Colloid J, 2011, 73(1): 118-127.

[77] Powers KW, Brown SC, Krishna VB, Wasdo SC, Moudgil BM, Roberts SM. Research strategies for safety evaluation of nanomaterials. Part VI. Characterization of nanoscale particles for toxicological evaluation. Toxicol Sci, 2006, 90(2): 296-303.

[78] Klang V, Valenta C, Matsko NB. Electron microscopy of pharmaceutical systems. Micron, 2013, 44: 45-74.

[79] Fan L, Wu H, Zhang H, Li F, Gu CH, Yang Q. Antitumor drug Paclitaxel-loaded pHsensitive nanoparticles targeting tumor extracellular pH. Carbohydr Polym, 2009, 77(4): 773-8.

[80] Babu PK, Oldfield E, Wieckowski A. Nanoparticle surfaces studied by electrochemical NMR. Modern Asp Electrochem, Springer, 2004, 36: 1-50.

[81] Gomez MV, Guerra J, Myers VS, Crooks RM, Velders AH. Nanoparticle size determination by (1)H NMR spectroscopy. J Am Chem Soc, 2009, 131(41): 14634-5.

[82] Cho CF, Ablack A, Leong HS, Zijlstra A, Lewis J. Evaluation of nanoparticle uptake in tumors in real time using intravital imaging. J Vis Exp, 2011, (52): pii: 2808.

[83] Jiang W, Kim BY, Rutka JT, Chan WC. Advances and challenges of nanotechnologybased drug delivery systems. Expert Opin Drug Deliv, 2007, 4(6): 621-33.

[84] Bertrand N, Wu J, Xu X, Kamaly N, Farokhzad OC. Cancer nanotechnology: the impact of passive and active targeting in the era of modern cancer biology. Adv Drug Deliv Rev, 2014, 66: 2-25.

[85] Venditto VJ, Szoka Jr FC. Cancer nanomedicines: so many papers and so few drugs. Adv Drug Deliv Rev, 2013, 65(1): 80-8. 

Chapter 4

\title{
Nanotechnologies Applied in Biomedical Vaccines
}

\author{
Yuan-Chuan Chen, Hwei-Fang Cheng, \\ Yi-Chen Yang and Ming-Kung Yeh \\ Additional information is available at the end of the chapter \\ http://dx.doi.org/10.5772/63453
}

\begin{abstract}
Vaccination, one of the most effective strategies to prevent infectious diseases, is the administration of antigenic materials to stimulate an individual's immune system to develop adaptive immunity to a specific pathogen. Though it is so advantageous for diseases control and prevention, vaccines still have some limitations. Nanotechnology is an approach to prepare a novel biomedicine vaccine with the vaccine consumption and side effects significantly decreased. Regulation is the most important criterion for the development of nanovaccines. All marketing products have to meet the requirement of regulation. The fast-track designation potentially aids in the development and expedites the review of nanovaccines that show promises in an unmet medical need. Here, somesuccessfulnanovaccine products areintroduced-Inflexal ${ }^{\circledR} \mathrm{V}, \mathrm{Epaxal}{ }^{\circledR}, \mathrm{Gardasi}{ }^{\mathrm{TM}}$ and Cervarix ${ }^{\mathrm{TM}}$ have been widely used for the clinical applications, which are delivered either in the form of virosomes or virus-like particles. Vaccines based on nanotechnology may overcome their original disadvantages and lead to the development of painless, safer, and more effective products.
\end{abstract}

Keywords: biomedicine, clinical application, fast track, nanotechnology, vaccine

\section{Introduction}

The terms vaccine and vaccination, derived from Variolae vaccinae (smallpox of the cow), were devised by Edward Jenner to denote cowpox. Edward Jenner was an English doctor and scientist who was the pioneer of smallpox vaccine, the first vaccine in the world. In 1796, Jenner took pus from the hand of a milkmaid with cowpox and scratched it into the arm of an 8-yearold boy. Six weeks later, he inoculated the boy with smallpox, afterwards observing that he did not catch smallpox and no diseases followed [1,2]. The boy was further challenged with 


\begin{tabular}{|c|c|c|}
\hline Type & Definition & Example \\
\hline Inactivated & $\begin{array}{l}\text { Virulent microbes are previously } \\
\text { destroyed with chemicals, heat, } \\
\text { radiation, or antibiotics }\end{array}$ & $\begin{array}{l}\text { Influenza vaccine, whole cell pertussis } \\
\text { vaccine, cholera vaccine, hepatitis A vaccine, } \\
\text { rabies vaccine, plague vaccine, inactivated } \\
\text { polio vaccine (IPV) }\end{array}$ \\
\hline Attenuated & $\begin{array}{l}\text { Live microbes are cultivated under } \\
\text { conditions to disable their virulence, } \\
\text { or closely related but less dangerous } \\
\text { microbes are used to induce broad } \\
\text { immune responses }\end{array}$ & $\begin{array}{l}\text { Yellow fever vaccine, shingles vaccine, } \\
\text { measles, mumps and rubella (MMR) vaccine, }{ }^{(1)} \text { typhoid } \\
\text { (Ty21a) vaccine, BCG vaccine, rotavirus vaccine, } \\
\text { live attenuated influenza vaccine (LAIV), } \\
\text { oral polio vaccine (OPV) }\end{array}$ \\
\hline Toxoid & $\begin{array}{l}\text { Vaccines are made from inactivated } \\
\text { toxic compounds that cause illness } \\
\text { rather than microbes }\end{array}$ & Tetanus toxoid, diphtheria toxoid \\
\hline \multirow[t]{3}{*}{ Subunit } & $\begin{array}{l}\text { Protein-based vaccine: A fragment of } \\
\text { protein creates an immune response, } \\
\text { rather than introducing inactivated } \\
\text { or attenuated microbes to an immune } \\
\text { system. }\end{array}$ & $\begin{array}{l}{ }^{(2)} \text { Hepatitis B vaccine, }{ }^{(2)} \text { human } \\
\text { papillomavirus (HPV) vaccine }\end{array}$ \\
\hline & $\begin{array}{l}\text { Polysaccharide vaccine: A } \\
\text { capsular polysaccharide antigen } \\
\text { is covalently attached to a } \\
\text { carrier protein and creates an immune } \\
\text { response. }\end{array}$ & $\begin{array}{l}{ }^{(1)} \text { typhoid }(\mathrm{Vi}) \text { vaccine, } \\
{ }^{(3)} \text { pneumococcus vaccine, } \\
{ }^{(4)} \text { meningococcus vaccine }\end{array}$ \\
\hline & $\begin{array}{l}\text { Conjugate vaccine: Certain } \\
\text { pathogens have poorly immunogenic } \\
\text { polysaccharide outer coats. The } \\
\text { immune system recognizes the } \\
\text { polysaccharide as if it were a } \\
\text { protein antigen by linking } \\
\text { these outer coats to proteins }\end{array}$ & $\begin{array}{l}\text { Haemophilus influenzae } \\
\text { type b (Hib) vaccine, }{ }^{(3)} \text { pneumococcus } \\
\text { vaccine, }{ }^{(4)} \text { meningococcus vaccine }\end{array}$ \\
\hline
\end{tabular}

Table 1. The major types of vaccines in the clinical application represent different strategies used to reduce the risk of illness, while retaining the ability to induce beneficial immune responses.

various materials and again showed no signs and symptoms. In 1798, Jenner extended his studies and reported that his vaccine was safe and could be transferred from arm-to-arm to reduce dependence on uncertain supplies from infected cows [3]. In the 1880s, the second generation of vaccines was introduced by Louis Pasteur who developed vaccines for chicken cholera and anthrax [4]. In 1881, to honor Jenner, Louis Pasteur proposed that the terms should be extended to cover the new protective inoculations then being developed [4]. From the late nineteenth century, vaccines were considered a matter of national prestige, and vaccination is a compulsory policy in many countries [5]. In the twentieth century, several successful vaccines were introduced such as those against diphtheria, measles, mumps, and 
rubella. During this period, the development of the polio vaccine in the 1950s and the eradication of smallpox around 1960s-1970s are great achievements. As vaccines had already become more common, many people began taking them for granted.

A vaccine, an antigenic material, is a biological preparation that provides adaptive immunity to a specific disease. The agent triggers immune responses to recognize the disease-causing agent to destroy and remember it, so that the immune system can immediately recognize and destroy the foreign agent once it invades into the body later. Inactivated microbes live attenuated microbes, toxoids, subunits, or conjugates have been manufactured as vaccines and employed to stimulate adaptive immune responses (Table 1). A monovalent (univalent) vaccine is designed to immunize against a single antigen or microorganism such as measles vaccine, while a multivalent (polyvalent) vaccine is designed to immunize against more than one strain of the same microorganism or against more than one microorganism such as oral polio vaccine (OPV), three in one (e.g., diphtheria-tetanus-pertussis, DTP) vaccine, etc. Heterologous vaccines are pathogens of other animals that do not cause diseases or only cause mild diseases in the organism being treated, such as the cowpox vaccine and the Bacillus Calmette-Guérin (BCG) vaccine.

(1) Two types of typhoid vaccines are available: typhoid (Ty21a) vaccine (a live, attenuated vaccine given by mouth) and typhoid (Vi) vaccine (a subunit vaccine given by injection). (2) Current hepatitis B vaccine and human papillomavirus (HPV) vaccine may be produced by recombinant DNA technology; therefore, they are sometimes called recombinant vaccines. (3) Two types of pneumococcus vaccines given by injection are available: pneumococcal polysaccharide vaccine (PPSV) and pneumococcal conjugate vaccine (PCV). (4) Two types of meningococcus vaccines given by injection are available: meningococcal polysaccharide vaccine (MPSV) and meningococcal conjugate vaccine (MCV).

It is well-known that prevention is better than cure for diseases control. Vaccination is the best approach to prevent infectious diseases ahead of the incidence of diseases. Vaccination can prevent or ameliorate morbidity from infection and result in herd immunity when a high percentage of population has been vaccinated. For the past centuries, some diseases are globally eradicated such as smallpox; some diseases are significantly controlled in much of the world such as polio, measles, and tetanus through vaccines distribution. The effectiveness of vaccination has been widely studied and verified such as the influenza vaccine [6], HPV (Human papillomavirus) vaccine [7], and the shingles (chicken pox) vaccine [8], etc. In 2012, the World Health Organization (WHO) reports that licensed vaccines are currently available to contribute to the prevention and control of 25 infections. From the discovery of the related pathogens, 25 infection have been effectively prevented using licensed vaccines; however, vaccines remain elusive for many important diseases, such as the infections of Zika viruses, cytomegaloviruses (CMV), hepatitis $C$ viruses (HCV), Human immunodeficiency viruses (HIV), severe acute respiratory syndrome (SARS) viruses, Ebola viruses, respiratory syncytial viruses (RSV), and malaria parasites Plasmodium, etc. (Figure 1). A vaccine is not only conventionally prophylactic, hinting to prevent or alleviate the effects of a future infection, but also may be therapeutic, vaccines against cancers are being investigated in recent research such 
as the HPV vaccine for cervical cancer, the BCG vaccine for bladder cancer [9] and colorectal cancer [10].

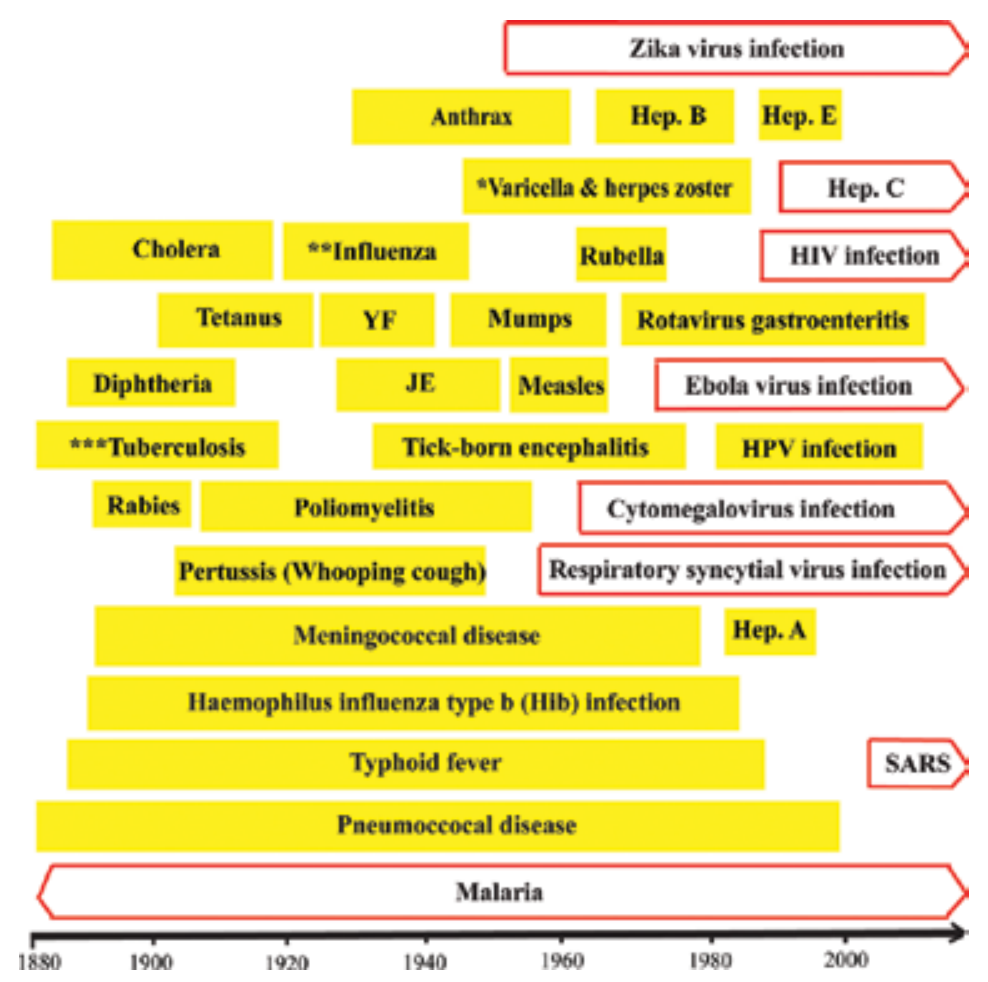

Figure 1. Time line for the development of vaccines: 25 infections ( yellow column) have been reported by WHO being prevented using licensed vaccines. However, some infections under research have no effective vaccines available yet ( $\square$ blank arrow column). *Varicella and herpes zoster: Varicella (chicken pox) and herpes zoster (shingles) are diseases caused by varicella-zoster viruses (VZV) (one of the eight herpesviruses known to infect humans and vertebrates). ${ }^{* *}$ Influenza: A new version of the influenza vaccine is usually developed twice a year, because influenza viruses mutate rapidly. ${ }^{* * *}$ Tuberculosis (TB): The Bacillus Calmette-Guérin (BCG) vaccine was originally developed from Mycobacterium bovis found in cows; therefore, its efficacy against pulmonary TB appears to be variable in humans. Abbreviations: Hepatitis (Hep.); Human papillomavirus (HPV); Human immunodeficiency virus (HIV); Japanese encephalitis (JE); Severe acute respiratory syndrome (SARS); Yellow fever (YF).

Vaccines have not only successfully reduced the incidence rate of many devastating diseases to be always low but also prevented many infectious diseases from persistently bursting out. At present, only few people have ever experienced the detrimental effects of many illnesses. The two main advantages of vaccination are noted as follows:

1. Prevention in advance

Vaccination can result in lower morbidity and mortality rate as well as faster recovery even though a partial, late, or weak immunity may only alleviate an infection. The early protective efficacy of vaccination is primarily conferred by the induction of antigenspecific antibodies. Vaccination can trigger adaptive immunity before contacting with the 
pathogen. Should a pathogen infect the body, there is no illness in that the immune responses stimulated by vaccines continuously guard against the disease. The quality of such antibody responses (avidity: a measure of the overall strength of an antigen-antibody complex) has been identified as a determining factor of vaccines efficacy. However, there is more to antibody-mediated protection than the peak of vaccine-induced antibody titers. Vaccination stimulates both humoral and cell-mediated immunity of one's own throughout the body. This kind of defensive response is usually safer, more effective and with fewer side effects (e.g., inflammation, muscle aches, allergy, etc.) due to the active, acquired and specific immunity is established. Through vaccination, humans can eliminate or alleviate the attack of pathogens beforehand to avoid the suffering of diseases.

2. Immunity for long term

Vaccination stimulates adaptive immunity to create immunological memory that can maintain in the body for a long period of time. Therefore, people will be protected from the attack of diseases as long as these specific memory cells are still in the body. The disease control or elimination requires the induction of protective immunity in a sufficient proportion of the population. This is best achieved by vaccination programs capable of inducing the long term protection (adaptive immunity) that contrasts to the quick but short-lasting immune responses (innate immunity). Long-term immunity is conferred by the maintenance of antigen-specific immune effectors and/or by the induction of immune memory cells that may be efficiently and rapidly reactivated upon subsequent microbial exposure.

In spite of vaccines' advantages for diseases control and prevention, three major limitations related to vaccines are noted as follows:

1. Complex vaccination schedules

The potency of vaccines may not be high enough in receiving immunization only once. Though the body develops antibodies, protection sometimes is not adequate due to the development of immunity might be too slow to be effective in time. Furthermore, the antibodies might not disable the pathogen completely, or there might be multiple strains of the pathogen, not all of which are equally susceptible to the immune responses. In order to provide the best protection, additional "booster" shots are often required to achieve full immunity. Vaccine schedules are usually regulated by the competent authority or physician groups to achieve maximum effectiveness. A vaccination schedule is a series of vaccinations, including the timing of all doses, which may be either recommended or compulsory. Many vaccines require multiple doses for maximum effectiveness, either to produce sufficient initial immune responses or to boost responses that fade over time. Over the past two decades, the recommended vaccination schedule has grown rapidly and become more complex as many new vaccines have been developed.

2. Strict requirements for storage and shipment

Vaccines are temperature sensitive and their storage and shipment are usually restricted to cold temperature [11]. Potency can be negatively affected by exposing to extended 
or multiple temperature excursions (out-of-range temperatures). It is essential to have a label on the storage unit to indicate that vaccines should be stored in the freezer $(-20$ or $-80^{\circ} \mathrm{C}$ ) or in the refrigerator $\left(2-8^{\circ} \mathrm{C}\right)$. Correct cold-chain (a temperature-controlled supply chain) procedures must be followed during production, warehousing, and transportation, that is, vaccines have to be stored properly from the time that they are manufactured, distributed until administered to the people. Most live, attenuated vaccines tolerate freezing temperatures, but deteriorate rapidly after being removed from cold temperature. Inactivated vaccines will be damaged by exposing to temperature fluctuations. Coldchain failure has to be evaluated in addition to routine tests such as potency, sterility, toxicity, safety, chemical, visual, and pyrogen test.

3. Restricted routes of administration

Most vaccine products are administered by injection such as intramuscular (IM), subcutaneous (SC), and intradermal (ID) injection, though some can be given by oral administration or intranasal spray. IM injection administers the vaccine into the muscle mass. Vaccines containing adjuvants should be injected into muscle to reduce adverse local effects. SC injection administers the vaccine into the subcutaneous layer above the muscle and below the skin. ID injection administers the vaccine in the topmost (dermis) layer of the skin. BCG is the known vaccine with ID injection; this route reduces the risk of neurovascular injury. The professional person's assistance is usually required for the injection administration. Oral administration vaccines (e.g., OPV and rotavirus vaccine) make immunization easier by self-administration and eliminating the need of an injector. The intranasal spray vaccine (e.g., influenza vaccine live) also offers a needle-free approach through the nasal mucosa. Percutaneous administration, such as a multiple puncture device (4-5 cm length, with 9 short needles) used for BCG vaccination in Japan, indicates that any medical procedure is done via needle puncture of the skin, rather than using an "open" approach where inner organs or tissues are exposed.

In the new era of subunit vaccines, to against both infectious diseases and cancers effectively, subunits made of proteins, peptides, DNA antigens, or potent adjuvants may provide a good choice to enhance immunogenicity. Subunit vaccines are not generally immunogenic in humans because of size, degradation, destruction, nonspecific targeting, lack of crosspresentation, and others [12]. This reveals that it is necessary to develop a new approach to package and present antigens to the immune system. Nanomedicine is an area of research that combines nanotechnology and medicine and provides new hopes for therapeutic strategies and diseases control.

Advances in nanotechnology have shown to be beneficial for therapeutic strategies such as drug discovery, drug delivery, and gene/protein delivery. Through nanotechnology, drug consumption and side effects can be significantly decreased by locating the active agent at the desired site. Nanotechnology is the study and application of extremely small things and capable of being used in many science fields, such as biology, chemistry, physics, materials science, and engineering. It is referred to the particular technology with a goal to precisely manipulate atoms and molecules for fabrication of nanoscale products, which size below 100 nm (or sometimes up to 200-300 nm) [12] (Figure 2). Advances in nanotechnology in biome- 
dicines have proved to be beneficial in vaccines development. The efficacy of vaccines can be improved and side effects also can be significantly decreased. For the past decades, a nanovaccine has become a novel approach for vaccination.

Adjuvants may be added to vaccines to modify the immune response by boosting it to give more antibodies and longer-lasting protection. Adjuvants have been an important component of vaccine formulations since aluminum salt (Alum) was approved in the 1920s [13] and adjuvant system 04 (AS04) was approved in 2009 in the United States, respectively. AS04 is a trade name for combination of adjuvants used in vaccine products by GlaxoSmithKline (GSK), which consists of aluminum hydroxide $\left(\mathrm{Al}(\mathrm{OH})_{3}\right)$ and 3-O-desacyl-4-monophosphoryl lipid A (MPLA). The toll-like receptor (TLR) agonist and MPLA are being intensively investigated as part of a new class of biological adjuvants [14]. In Europe, a few other adjuvants based on oil in water emulsions such as adjuvant system 03 (AS03) and liposomes (made of lipid vesicles) have been approved for using as adjuvants in humans by the European Medicines Agency (EMA) since the 1990s [15]. AS03 is a trade name for a squalene-based immunologic adjuvant used in vaccine products by GSK, which contains squalene, DL- $\alpha$-tocopherol and polysorbate 80 . For a great need for potent adjuvants in the vaccine clinical applications, we believe that nanotechnology could be well positioned to take on such a challenge. Consequently, we focus on the vaccine products based on nanotechnology. In this chapter, we review the regulation related to the development of nanovaccines, already marketed products based on nanotechnology and the perspective of developing highly effective, stable, and economic vaccines using micro-/nanoparticles.

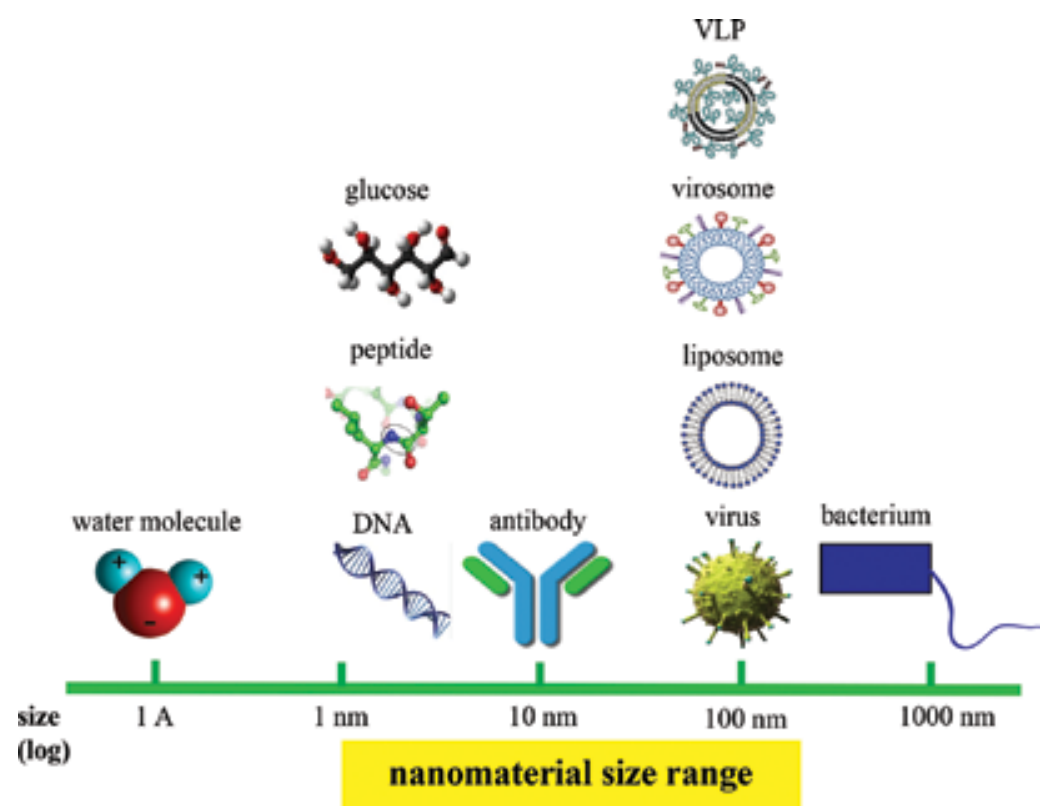

Figure 2. Size comparison of biological substances: The size range of nanomaterials used in nanotechnology relative to the size of various biological system. Abbreviation: virus-like particle (VLP). 


\section{Regulation}

In accordance with the guidelines of WHO, US Food and Drug Administration (FDA) and EMA, vaccines belong to a heterogeneous class of agent; each has suitable, individual, and specific standards of evaluation. The major quality concerns in a vaccine human clinical trial are as follows:

1. Theoretical values and expected values of the trial: In preclinical studies stage, potency, and immunogenicity should be tested in addition to toxicity and safety. A suitable animal challenging model should be established; otherwise, an alternative method must be proposed. The contribution and clinical significance of vaccine should be explained, and a declaration of interest is usually needed.

2. Protection of the testers: The right and interest of testers should be emphasized either in the placebo control group (e.g., use insert placebos) or the active control group (e.g., use already licensed vaccines).

3. Selection of testing population group: Random health volunteers are tested in phase I, but the representation of testers' population should be considered in phase II and III. The related factors include testers' population, social economics, and the epidemiology of target disease, etc.

4. Condition of inclusion and exclusion for testing population group: Tester should be in good health condition and meet the criteria of examination, some people would be excluded such as allergy, cardiovascular disease, immunodeficiency, and neuropathy sicks.

5. Dosage, administration route, and schedule: Dose-response data should be achieved in the dose-finding study. The schedule of vaccination depends on the traits of antigens, the characteristics of testing population, and the pharmacokinetics induced by vaccines. Tolerance, safety, dosage, and administration routes should be evaluated in phase I. The optimal dose and schedule of vaccination should be defined in phase II and would be a reference in phase III.

6. Safety evaluation of testers: The adverse events should be recorded and evaluated in all phases of the clinical trial. Post-marketing surveillance for side effects in phase IV is usually required.

7. Limitation of concomitant administration: The interaction between drugs and vaccines is rare, but it is necessary to record the consequence while concomitant administering them.

8. Efficacy and effectiveness of vaccines: Vaccine efficacy is the measure of the reduced disease incidence after vaccination, compared with the unvaccinated group. Vaccine effectiveness is the measure of vaccine induced protection (not population related protection), which is influenced with many factors such as the protection coverage of vaccination, the immunological state of testers, and the epidemiology of target disease. 
9. Lot-to-lot consistency study: It is necessary to evaluate the consistency of vaccine production and performance by comparing the different lots of vaccine products by the predefined standard (usually various parameters in immunology).

Vaccines, as all medicinal products regulated by national regulatory authorities (NRA) and national control laboratories (NCL), undergo a rigorous review of laboratory and clinical data to ensure their safety, efficacy, purity, and potency. In the stages of discovery and development of vaccines, the manufacturer has to comply with the related regulation, including nonclinical work, consultation, preclinical trials, and human clinical trials. Furthermore, vaccines approved for marketing may also be required to perform additional studies to give further evaluation and often address specific questions such as safety, efficacy or possible side effects and contraindications for post-marketing surveillance (Figure 3).

Lot release is an independent assessment of each lot of a licensed vaccine or biologic prior to it is released onto the market. General practices for lot release involve the review of manufac-

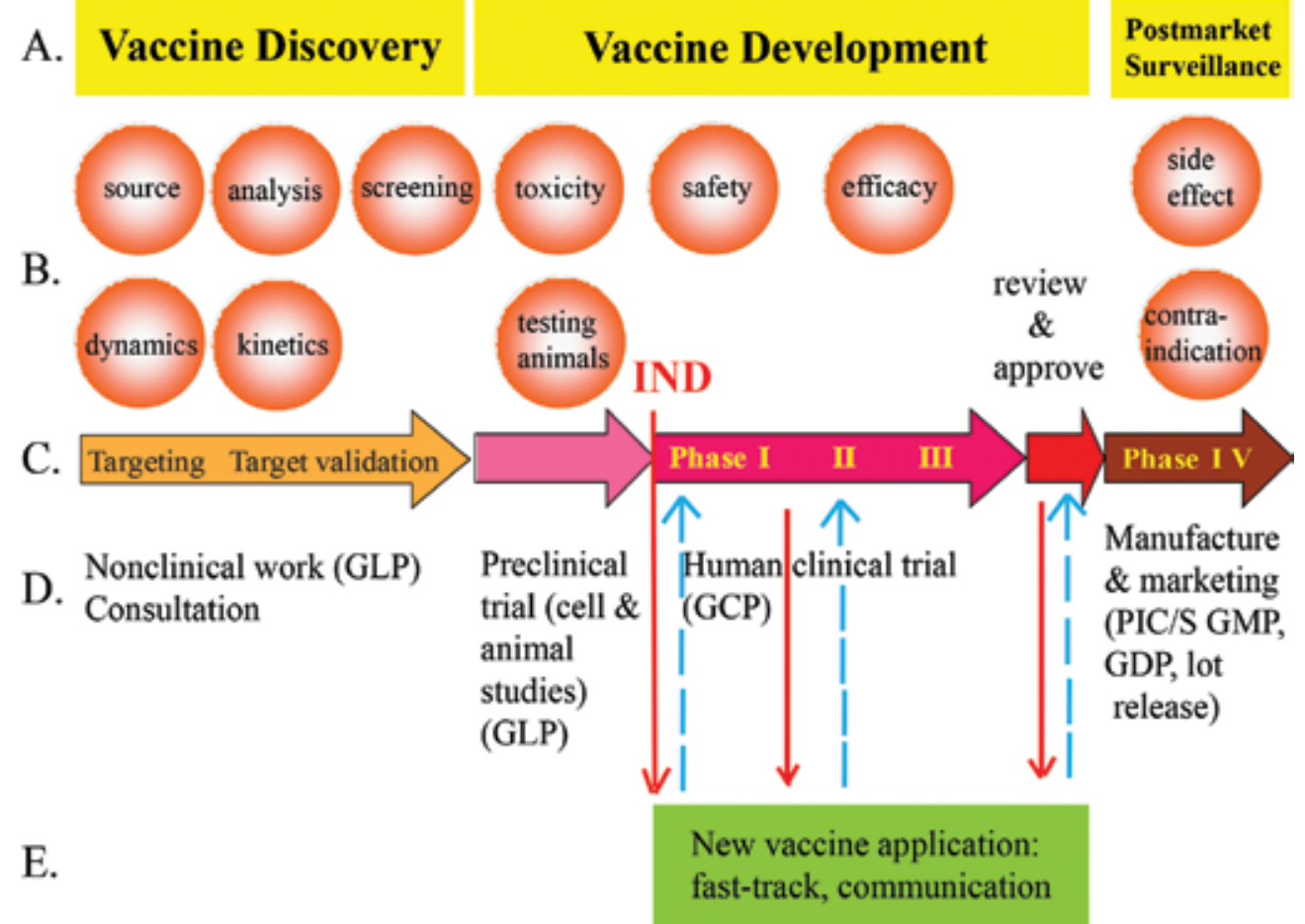

Figure 3. Frame work of vaccine research and development: The research, development, surveillance, and application of vaccines involve several practices performance and regulation requirements. (A) Stage: Vaccines discovery, development, and postmarked surveillance are three stages for the application of new vaccines. (B) Studies: There is much research work to do at every stage. (C) Phase: Each stage can be divided into different phases. (D) Regulation: Various regulations or guidelines are required for every stage. (E) Special procedure: fast track and communication. Abbreviations: Good Laboratory Practice (GLP); Good Clinical Practice (GCP); Good Distribution Practice (GDP); Investigational New Drug (IND), Pharmaceutical Inspection Convention and Pharmaceutical Inspection Co-operation Scheme/ Good Manufacturing Practice (PIC/S GMP). 
turers' production data and quality control test results by NRA and NCL. These practices can be performed by an agency or a contracted laboratory for the NRA. It may be supplemented by other documents such as the release certificate from the responsible NRA/NCL and in some circumstances, by tests in the other laboratory which is independent of the manufacturers' quality control testing.

Fast-track procedures can be implemented under special circumstances, such as disease outbreaks or an acute shortage in global vaccine supply. It is a process designed to facilitate the development and expedite the review of drugs that show promise in treating a serious or life-threatening disease and address an unmet medical need as follows:

1. Serious condition: To determine if the drug will have an impact on such factors as survival, day-to-day functioning or the disease will progress more serious if left untreated such as acquired immune deficiency syndrome (AIDS), Alzheimer's disease, heart failure, cancer, epilepsy, depression, and diabetes.

2. Unmet medical need: The drug must be developed to treat or prevent a disease that does not have a current therapy. If there are available therapies, a fast-track drug must show some advantage over available therapy such as showing of superior effectiveness, avoidance of serious side effects, improvement of the diagnosis, decrease of clinically significant toxicity, and the need of emerging or anticipated public health.

The purpose of a fast-track designation is to get important new drugs to the patient earlier through the consultation system. Once a drug receives fast-track designation, early and frequent communication between the NRA and a drug manufacturer is encouraged throughout the entire development and review process of drugs. The communication ensures that all questions and issues can be solved quickly, often leading to earlier drug approval and access by patients. For example, US FDA is able to offer accelerated approval or priority review if the requisite criteria are met. Priority review rapids the review process for a new drug from 6 to 10 months even can have US FDA make a final decision within 60 days.

In the application of nanoparticles as vaccines, some obstacles need to be overcome such as stability during manufacturing and storage, sterilization by nonthermal methods, and reproducibility of formulation during large-scale production [16]. Reproducibility of formulation during manufacturing is one of the major hurdles [17]; for instance, size-dependent immunogenicity has been reported [18] and it is observed that nanomaterials may change toxicity only by changing size and shape but not composition [19]. If the nanoparticles are cleared slowly over a long period of time, they may induce toxic effect. In addition, large counterparts may accumulate in vital organs to cause toxicity, even though small nanoparticles can be removed quickly from the body. Because of the toxic concerns of nanomaterials, the potential effects on the human body, public health, and environment pollution should be taken into account. It is necessary to evaluate the effects on DNA damage, gene expression, cell death, and growth in the body when different types of cells are exposed to nanoparticles. The safety surveillance and quality control of nanovaccines are as central as the evaluation of their efficacy. Regulatory policies are based mainly on the purity and safety of the vaccine. For 
risk management, some special regulations or guidelines for nanotechnology application in vaccines are as follows.

1. The approach to nanotechnology products
A. Product-focused and science-based regulatory policy
B. Attention to nanomaterials being incorporated into standing procedures
C. In legal standards for different product-classes

2. The regulation of pharmacopoeia (European Pharmacopeia 7.0 and 8.0; EP7.0, 8.0)

A. General provisions of products: The production method is validated to demonstrate that the product would comply with the test for abnormal toxicity for immunosera and vaccine for human use. Additionally, this method has to yield consistent vaccines comparable with the vaccine of proven clinical efficacy and safety in humans.

B. Preparation of nanoparticles (virosomes): Inactivated influenza virions are solubilized using a suitable detergent and purified by high-speed centrifugation to obtain supernatants containing mainly influenza antigens. After the addition of suitable phospholipids, virosomes are formed by removal of the detergent either by adsorption chromatography or another suitable technique.

C. Tests of final products: residual infectious virus, $\mathrm{pH}$, phospholipids, ratio of hemagglutinin to phospholipids, antimicrobial preservative, free formaldehyde, ovalbumin, total protein, sterility, virosome size, and bacterial endotoxin

3. Preclinical efficacy and toxicity testing of nanoparticles

A. Physicochemical characterization: size measurement, topology, molecular weight, aggregation, purity, chemical composition, surface characteristics, functionality, zeta potential, stability, and solubility test

B. In vitro characterization: sterility, targeting, drug release, in vitro immunology, and toxicity test

C. In vivo characterization: efficacy and repeated-dose toxicity test

\section{Products in the clinical application using micro/nanoparticles}

Inflexal® $\mathrm{V}$, Epaxal ${ }^{\circledR}$, Gardasil $^{\mathrm{TM}}$, and Cervarix ${ }^{\mathrm{TM}}$, marketed in many countries for over 10 years, are four common vaccine products based on micro/nanotechnologies. They use nanoparticles-virosomes (special liposomes) or virus-like particles (VLPs) as a delivery and adjuvant system, and all should be administered by IM and stored at $2-8^{\circ} \mathrm{C}$ (Table 2). 


\begin{tabular}{llll}
\hline Product & Inflexal ${ }^{\circledR}$ V & Epaxal ${ }^{\circledR}$ & ${ }^{\left({ }^{(1)}\right.}$ Gardasil $^{\mathrm{TM}}$ and $^{(2)}$ Cervarix $^{\mathrm{TM}}$ \\
\hline Indication & Influenza & Hepatitis A & Cervical cancer \\
Formulation & a mixture of three & a vaccine adjuvanted & ${ }^{(1)}$ Aluminum adjuvant \\
& monovalent virosome & with IRIV & composed of AAHS \\
& pools & & ${ }^{(2)} \mathrm{AS} 04$ adjuvant composed \\
& & of Al $(\mathrm{OH})_{3}$ and MPLA \\
Nanoparticle & Virosome & Virosome & VLP \\
Size in diameter & $150 \mathrm{~nm}(100-300 \mathrm{~nm})$ & $150 \mathrm{~nm}(100-300 \mathrm{~nm})$ & $20-60 \mathrm{~nm}$ \\
\hline
\end{tabular}

Abbreviations: adjuvant system 04 (AS04); amorphous aluminum hydroxyphosphate sulfate (AAHS); aluminum hydroxide $\left(\mathrm{Al}(\mathrm{OH})_{3}\right)$; immunopotentiating reconstituted influenza virosomes (IRIV); 3-O-desacyl-4-monophosphoryl lipid A (MPLA); virus-like particle (VLP).

Table 2. Comparison of different vaccine products based on nanotechnology.

A virosome is a kind of vaccine delivery tool with a typical size in diameter about $150 \mathrm{~nm}$. It consists of a phospholipid membrane vesicle incorporating viral-derived proteins and tends to form a pure fusion-active vesicle. Virosomes are only able to fuse with target cells without replicating inside their hosts. In contrast to liposomes, virosomes contain functional viral envelope glycoproteins: influenza virus hemagglutinin (HA) and neuraminidase (NA) intercalated in the phospholipid bilayer membrane. Virosomes represent an innovative, extensively applicable adjuvant and carrier system and are used in areas beyond conventional vaccines.

Virus-like particles (VLPs), derived from viruses and having no genetic materials, are selfassembling nanoparticles (20-60 $\mathrm{nm}$ in diameter) that expose multiple epitopes on their surface and resemble native virions [20,21]. VLPs can differentiate themselves from soluble recombinant antigens, and their structure is capable of inducing stronger immune responses. They also can express one or several viral structural proteins in a recombinant heterologous system [22], for example, the recombinant baculovirus system results in the expression of massive and various virus proteins. It is possible to engineer vaccines with multiple viral epitopes to elicit protective immunity due to the availability of viral three-dimensional structures [23]. VLPs co-administered with oil, mutant E. coli heat-labile toxins in calves [24] and with bluetongue viruses in sheep [25] have provided protection against the related viruses. The immunogenicity of VLPs may be induced because of their interaction with dendritic cells (DCs) and has been a useful approach for immunity against viruses in animals [16].

\subsection{Inflexal® V}

Influenza is an important respiratory infection of humans, responsible for many deaths worldwide every year. The influenza viruses are genetic variable due to continuous and gradual mutation as well as rearranged genome segments between viruses [26]. Frequent mutations of the surface glycoproteins HA and NA result in the gradual evolution of viral strains (antigenic drift). Consequently, it is required to develop a version of new influenza 
vaccine almost every year in that infectious viruses can no longer be neutralized effectively by the antibodies raised against previously circulating strains.

Inflexal ${ }^{\circledR} \mathrm{V}$, an influenza vaccine, is composed of a mixture of three monovalent virosome pools, each formed with specific glycoproteins HA and NA of one influenza virus strain. The vaccine is completely biodegradable and contains no thiomersal, formaldehyde, and very low levels of ovalbumin. Virosomes, reconstituted by nanotechnology, can deliver influenza antigens to stimulate a strong immune response; thus, this product is highly efficacious by mimicking natural viral infection [22]. The product has been approved by many countries and is the only adjuvanted influenza vaccine licensed for all age groups.

\subsection{Epaxal®}

Hepatitis A is an acute infectious disease of the liver caused by the hepatitis A virus (HAV), which symptoms include nausea, vomiting, diarrhea, jaundice, fever, and abdominal pain. Around $10-15 \%$ of people experience a recurrence of symptoms during the six months after the initial infection. Acute liver failure may rarely occur with this being more common in the elderly.

Epaxal®, a hepatitis A vaccine, is adjuvanted with immunopotentiating reconstituted influenza virosomes (IRIV). This vaccine is based on formalin inactivated HAV adsorbed to the surface of virosomes that replace the adjuvant-aluminum hydroxide with phospholipids. A single injection of virosomal HAV vaccine is well tolerated and highly immunogenic, with $88-97 \%$ of seroprotection 2 weeks after the first dose (reinforcing immunization: one dose at 6-12 months after the first dose).

\subsection{Gardasil ${ }^{\mathrm{TM}}$ and Cervarix ${ }^{\mathrm{TM}}$}

HPV is a sexually transmitted infection and results in serious illness including genital warts and cervical cancers. Cervical cancer is the second most common cause of cancer deaths in females worldwide, only next to breast cancer [27]. HPV causes many cervical cancer cases every year, of all the cases, type 16 and 18 are responsible for about $70 \%$.

Gardasil $^{\mathrm{TM}}$ and Cervarix ${ }^{\mathrm{TM}}$ are subunit vaccines containing VLPs assembled from the major capsid protein (L1 protein) of HPV type 6, 11, 16, and 18 (Gardasil ${ }^{\mathrm{TM}}$ ) and type 16 and18 $\left(\right.$ Cervarix $\left.^{\mathrm{TM}}\right)$ [21]. Vaccination with these two products has been shown to protect women against a high proportion of precursor lesions of cervical cancer caused by HPV.

Gardasil ${ }^{\mathrm{TM}}$ is a sterile liquid suspension prepared from the highly purified VLPs composed of L1 proteins of HPV types 6, 11, 16, and 18. The L1 proteins of these HPV types $(6,11,16$, and 18 ) are produced by separate fermentations in the recombinant Saccharomyces cerevisiae and self-assembled into VLPs. The VLPs for each type are purified and adsorbed on a aluminumcontaining adjuvant formulation-amorphous aluminum hydroxyphosphate sulfate (AAHS).

Cervarix $^{\mathrm{TM}}$ is a preparation of purified VLPs composed of L1 proteins of HPV type 16 and 18, which is formulated in AS04 adjuvant-containing aluminum hydroxide and 3-O-desacyl-4monophosphoryl lipid A (MPLA) [28]. The MPLA immunostimulant is a detoxified deriva- 
tive of the lipopolysaccharide of the bacterium Salmonella minnesota strain. The L1 proteins of these HPV types (16 and 18) are separately produced using a recombinant baculovirus expression system and the insect cell line derived from the moth-cabbage looper (Trichoplusia ni). After the expression of L1 proteins and further purification, L1 proteins are assembled separately as VLPs. It is the first vaccine licensed by the US FDA that includes MPLA as an adjuvant. The adjuvant AS04 acts as a substance incorporated into a vaccine that enhances or directs the immune response of the vaccinated individual.

The advantages that nanotechnology-based vaccines may over conventional vaccines are as follows:

1. Substitution for traditional adjuvants: Nanovaccines demonstrated immunogenicity similar to conventional vaccines without the presence of traditional adjuvant (Alum) which is found to cause irritation.

2. Extended history of safety and shelf life: Nanoparticles as vaccine delivery systems avoid the use of toxic substances such as thimerosal and formaldehyde and potentially extend the stability of vaccines on the shelf.

3. Less frequency of administration for boost: Nanovaccines can be administered for reinforcement immunization less frequently than conventional vaccines, which are usually multi-injection or multi-dose delivery systems.

\section{Perspective}

Nanotechnology may provide us a new choice for safe and effective vaccines. However, frequency of boost, refrigeration of vaccines, and routes of administration are still challenges for vaccines distribution in small areas or villages of some countries. Nanovaccines are expected to be more effective, convenient, and economic than conventional vaccines (Figure 4). We will have a great future ahead in nanovaccines if the following three aims are achieved.

1. Controlled release at specific location

Biodegradable polymers are superior to nondegradable polymers because the former may not need additional removal procedures. For reliability and reproducibility, synthetic biodegradable polymers are the best choice for antigen encapsulation in single-dose vaccine production. Biodegradable polymer microsphere such as poly-1-lactic acid (PLA) and poly lactic-co-glycolic acid (PLGA) has been used to control the time and rate of vaccine antigen release due to their safety and ability to provide long-term controlled vaccine antigen release [29]. Sustained release and pulsatile release are two possible types of vaccine antigen release with biodegradable PLGA microspheres. Sustained release, continued vaccine antigen diffusion after the initial release, mimics the administration of several small boosters. Pulsatile release, second vaccine antigen diffusion distinct from the first release, mimics the current vaccination schedule [30]. PLA and PLGA allow encapsulation within a hydrophobic core or adsorption to the hydrophilic shell, which 
can manipulate to encapsulate vaccine within the interior [31, 32]. For example, tetanus toxoid (TT) can be used to prevent tetanus which is caused by the bacterium Clostridium tetani. A pattern of constant release with a decreasing release rate after the initial burst of TT has been identified. Small-sized TT-PLA microspheres with rapid release kinetics induced an earlier release compared with larger TT-PLGA 50:50 microspheres with slow release kinetics [33]. A continuously increasing release rate after the initial burst was observed with low molecular weight TT-PLGA microspheres [34].

Nanoscale carriers can enhance the potency of vaccines to develop safer and more effective vaccine formulations. A nanoparticle-based strategy was demonstrated to safely deliver and detain intact toxins to mount a potent anti-toxin immune response. A biomimetic nanoparticle cloaked in biological membranes was used to sequester membrane-active toxins. As the event of contacting with the nanoparticles, the toxins will retrained and lose toxicity in that they are prevented from interacting with cellular targets. The resulting particles and toxin complex adopts a nanoparticulate morphology that facilitates the intracellular delivery of toxins. This technique assists to design a novel toxoid vaccine that promises more effective anti-toxin immune responses. The potent nanotoxoid formulations provide a viable anti-virulence approach to combat with microorganisms that contain membrane damaging toxins such as Staphylococcus aureus and Group A streptococcal infections [35].

2. Stable at room temperature

The common adjuvant -alum is known to cause irritation if it is used in the conventional hepatitis B vaccine. However, the use of needle-free nasal immunization with a combination of nanoemulsion and hepatitis B antigen was found to be tolerable, effective, and safe without side effects [36]. The solvent extraction or evaporation from a waterin-oil-in water $(\mathrm{W} / \mathrm{O} / \mathrm{W})$ emulsion is usually used to prepare the antigen-encapsulated nanoparticles [37]. Interestingly, refrigeration was not required for this nanoemulsion, as it was effective at $25^{\circ} \mathrm{C}$ for one month and at $40^{\circ} \mathrm{C}$ for 6 weeks, respectively [16].

To make an effort to develop nanoparticles from biodegradable and biocompatible polymers (e.g., PLA, PLGA) as vaccine delivery systems can induce both humoral and cell-mediated immunity. These biodegradable polymers may make nanovaccines have extended history of safety and proven biocompatibility at room temperature. The additional advantage is their property to control the time and rate of polymer degradation and antigen release [38].

3. Replaceable routes of administration

In addition to injection (IM, SC or ID), mouths and nostrils are two successful alternative routes for administration. The vaccine can be given by these two routes without the help of trained health workers and the equipment of sterile injection. These two methods are relatively effective, inexpensive, and convenient, compared with injection. For example, Sabin vaccine (OPV) and FluMist ${ }^{\circledR}$ are administered by oral administration and intranasal spray, respectively, in contrast to Salk vaccine (IPV) and influenza vaccine (flu shots), which are both given by injection. 
A. Sabin vaccine: An OPV is indicated for the prevention of poliomyelitis, which trivalent contains a mixture of attenuated strains of three poliovirus types. The live polioviruses vaccine produces antibodies to all types of poliovirus and triggers a local immune response in the mucous membrane of the intestines. Once infected with wild polioviruses, these antibodies protect against paralysis by restricting the viral replication inside the intestine to avoid invading to the nervous system. Later, the live attenuated vaccine virus would be excreted in

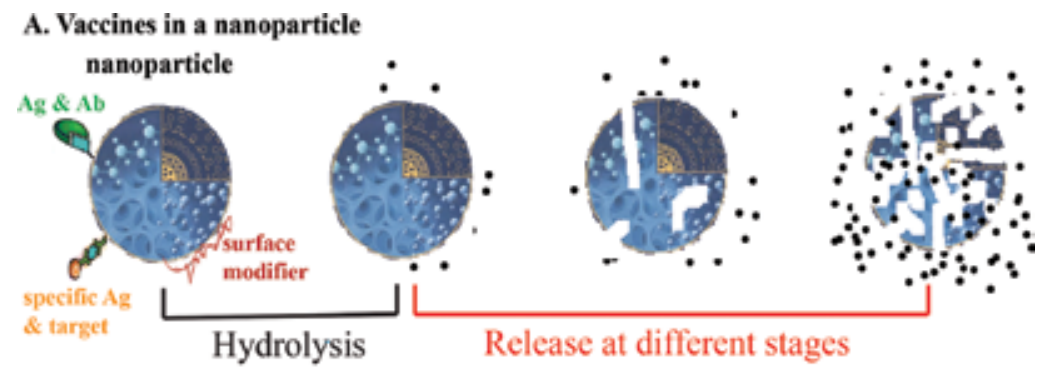

\section{B. Vaccine boost}

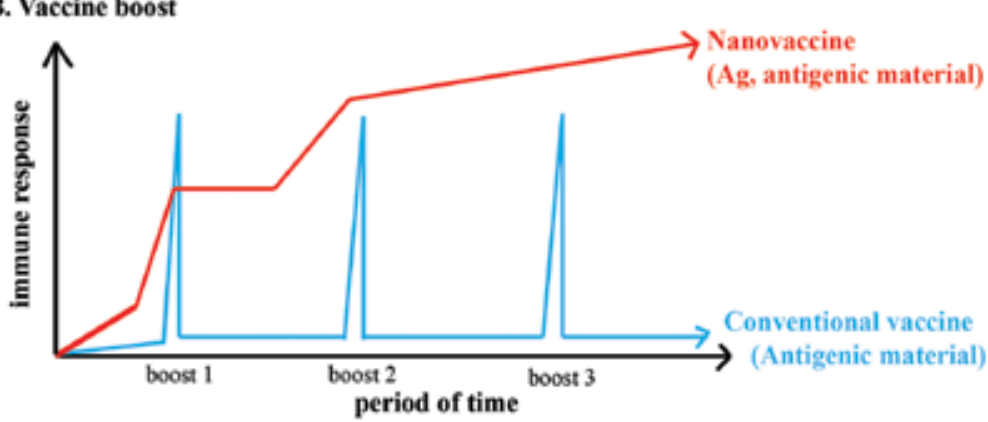

\section{Interaction of nanovaccines with the immune responses}

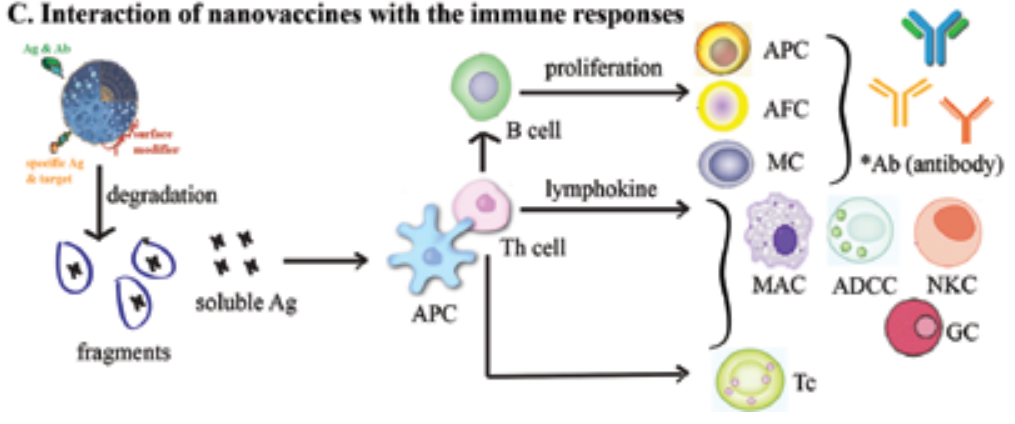

Figure 4. Nanotechnologies applied in biomedicines' vaccine (a cartoon figure for the perspective): Nanoparticles used in the vaccine production potentially overcome the disadvantages of conventional vaccines. (A) Vaccines encapsulated in nanoparticles which are polymers in solid form may be assistance in the stabilization at room temperature, elongation of storage time, exploration of alternative administration routes, and facilitation of controlled release. (B) Nanovaccines may only need to be boosted once; however, conventional vaccines usually need to be boosted many times. (C) Soluble antigens released from nanovaccines may induce both humoral immunity (B cell responses) and cell-mediated immunity (helper and cytotoxic T cell responses). ${ }^{*} \mathrm{Ab}$ (antibody): serum immunoglobulin G (IgG), mucosal IgG and mucosal IgA. Abbreviations: antigen (Ag); antibody (Ab); antigen presenting cell (APC); antibody forming cell (AFC); antibody-dependent cytotoxic cell (ADCC); cytotoxic T cell (Tc); granulocyte (GC); helper T cell (Th); macrophage (MAC); memory cell (MC); natural killer cell (NKC). 
the faces and spreaded to others in close contact. This means that OPV may result in the passive immunization of unvaccinated people, so that OPV can rapidly stop person-to-person transmission of wild poliovirus in areas where there is poor public health.

B. FluMist ${ }^{\circledR}$ : An intranasal spray attenuated vaccine is indicated for the prevention of influenza which quadrivalent contains four virus strains (a type A/H1N1, a type A/H3N2, and two type B strains). The live, attenuated influenza vaccine (LAIV) contains neither thimerosal nor preservatives and is sprayed into the nostrils. LAIV produces a significantly stronger immune response than inactivated vaccines, because it is delivered via the natural site of entry of the influenza virus [39-41]. In addition, this intranasal spray vaccine can prevent more $50 \%$ cases than the flu shots given by IM in younger children [42].

The skin is also an attractive organ for vaccine delivery because of its large surface area that is easily accessible for administration, and dermal delivery is almost pain-free [43, 44]. Furthermore, the skin contains many antigen presenting cells (e.g., Langerhans cells and dermal dendritic cells) and is available for the induction of immune responses [44-46]. Besides, the delivery via the skin circumvents the problem of swallowing, the first-pass effect of the liver, and the absorption and stability of vaccines in the gastrointestinal tract. However, the stratum corneum (the outermost layer of the epidermis) barrier may form significant resistance for vaccines to permeate into the body. To overcome the stratum corneum barrier, percutaneous administration such as microneedle arrays (needle-like structures with a length of less than $1 \mathrm{~mm}$ ) [43-46] is one of the most promising delivery systems.

Microparticles or nanoparticles (e.g., PLA, PLGA) have been proved to be capable of making an inactive Vibrio cholera whole-cell vaccine given by oral administration and demonstrating in vitro release in mice [47]. This is an approach using nanoparticles as an oral delivery system to improve bioavailability of vaccine and elated efficacy in therapy. Nanoparticulate material makes it possible that vaccines can be given without invasion through oral, nasal spray, and percutaneous administration such as diffusion patches, multiple puncture devices, and microneedle arrays, thus allowing painless and efficient delivery without the involvement of the professional.

\section{Conclusion}

Nanotechnology has extensively been utilized in the drug preparation and delivery for clinical applications. Nanomedicine has already been an attractive research area and the development of nanovaccines is one of the most promising enterprises. A variety of nanoparticles in vaccines have been employed as a delivery tool or immunity booster, inducing not only the highest efficacy but also the least side effects. The significance of nanovaccine development is to make it practical to have a perfect vaccination system including refrigeration-free, singledose, and painless administration. Indeed, we might have effective, safe, convenient, and cheap vaccines through nanotechnology in the near future. 


\section{Author details}

Yuan-Chuan Chen ${ }^{1}$, Hwei-Fang Cheng ${ }^{1}$, Yi-Chen Yang $^{1}$ and Ming-Kung Yeh $^{2,3^{*}}$

*Address all correspondence to: mkyeh2004@gmail.com

1 Food and Drug Administration, Ministry of Health and Welfare, Taipei, Taiwan

2 School of Pharmacy, National Defense Medical Center, Taipei, Taiwan

3 Ministry of Health and Welfare, Taipei, Taiwan

\section{References}

[1] Stern AM, Markel H. The history of vaccines and immunization: familiar patterns, new challenges. Health Aff. 2005; 24(3): 611-621. doi:10.1377/hlthaff.24.3.611

[2] Dunn PM. Dr. Edward Jenner (1749-1823) of Berkeley, and vaccination against smallpox. Arch. Dis. Child. Fetal Neonatal Ed. 1996; 74(1): F77-78. doi:10.1136/fn. 74.1.F77

[3] Baxby, D. Edward Jenner's Inquiry: a bicentenary analysis. Vaccine. 1999; 17(4): 301307. doi:10.1016/s0264-410x(98)00207-2

[4] Pasteur, L. Address on the germ theory. Lancet. 1881; 118(3024): 271-272. doi:10.1016/ s0140-6736(02)35739-8

[5] Stern AM, Markel H. The history of vaccines and immunization: familiar patterns, new challenges. Health Aff. 2005; 24(3): 611-621. doi:10.1377/hlthaff.24.3.611

[6] Fiore AE, Bridges CB, Cox NJ. Seasonal influenza vaccines. Curr. Top. Microbiol. Immunol. 2009; 333: 43-82. doi:10.1007/978-3-540-92165-3_3

[7] Chang Y, Brewer NT, Rinas AC, Schmitt K, Smith JS. Evaluating the impact of human papillomavirus vaccines. Vaccine. 2009; 27(32): 4355-4362. doi:10.3129/i09-126

[8] Liesegang TJ. Varicella zoster virus vaccines: effective, but concerns linger. Can. J. Ophthalmol. 2009; 44 (4): 379-384. doi:10.3129/i09-126

[9] Houghton BB, Chalasani V, Hayne D, Grimison P, Brown, CS, Patel, MI, Davis, ID, Stockler, MR. Intravesical chemotherapy plus bacille Calmette-Guérin in non-muscle invasive bladder cancer: a systematic review with meta-analysis. BJU Int. 2013; 111(6): 977-983. doi:10.1111/j.1464-410x.2012.11390.x

[10] Mosolits S, Nilsson B, Mellstedt H. Towards therapeutic vaccines for colorectal carcinoma: a review of clinical trials. Expert Rev. Vaccines. 2005; 4(3): 329-350. doi: 10.1586/14760584.4.3.329 
[11] Guidelines on the international packaging and shipping of vaccines. WHO/IVB/05.23, Printed: Dec. 2005. Available from: www.who.int/vaccines-documents/

[12] Mamo T, Poland GA. Nanovaccinology: the next generation of vaccines meets 21st century materials science and engineering. Vaccine. 2012; 30(47): 6609-6611. doi: 10.1016/j.vaccine.2012.08.023

[13] Kool M, Fierens K, Lambrecht BN. Alum adjuvant: some of the tricks of the oldest adjuvant. J. Med. Microbiol. 2012; 61(July (Pt 7)):927-934. doi:10.1099/jmm.0.038943-0

[14] Mbow ML, De GE, Valiante NM, Rappuoli R. New adjuvants for human vaccines. Curr. Opin. Immunol. 2010; 22(3): 411-416. doi:10.1016/j.coi.2010.04.004

[15] O'Hagan DT, De GE The path to a successful vaccine adjuvant - 'the long and winding road'. Drug Discov. Today. 2009; 14(11-12): 541-551. doi:10.1016/j.drudis.2009.02.009

[16] Nandedkar TD. Nanovaccines: recent developments in vaccination. J. Biosci. 2009; 34(6): 995-1003. doi:10.1007/s12038-009-0114-3

[17] Sharma S, Mukkur TK, Benson HA, Chen Y. Pharmaceutical aspects of intranasal delivery of vaccines using particulate systems. J. Pharm. Sci. 2009; 98(3): 812-893. doi: 10.1002/jps.21493

[18] Fifis T, Gamvrellis A, Crimeen-Inwin B, Pietersz GA, Li J, Mottram PL, McKenzie IFC and Plebanski M. Size-dependent Immunogenicity: therapeutic and protective properties of nanovaccines against tumors. J. Immunol. 2004; 173(5): 3148-3154. doi: 10.4049/jimmunol.173.5.3148

[19] Zhang T, Stilwell JL, Gerion D, Lianghao D, Elboudwarej O, Cooke PA, Gray JW, Alivisatos PA and Chen FF. Cellular effect of high doses of silica-coated quantum dot profiled with high throughput gene expression analysis and high content cellomics measurements. Nano Lett. 2006; 6(4): 800-808. doi:10.1021/n10603350

[20] Zhao Q, Li S, Yu H, Xia N, Modis Y. Virus-like particle-based human vaccines: quality assessment based on structural and functional properties. Trends Biotechnol. 2013; 31(11): 654-63. doi:10.1016/j.tibtech.2013.09.002

[21] Uddin MN, Kouzi SA and Hussain MD. Strategies for developing oral vaccines for human papillomavirus (HPV) induced cancer using nanoparticle mediated delivery system. Pharm. Pharm. Sci. 2015; 18(2): 220-234. Available from: http:// www.cspsCanada.org

[22] Kushnir N, Streatfield SJ, Yusibov V. Virus-like particles as a highly efficient vaccine platform: diversity of targets and production systems and advances in clinical development. Vaccine. 2012; 31(1): 58-83. doi:10.1371/j. vaccine.2012.10.083

[23] Roy P, Sutton G. New generation of African horse sickness virus vaccine based on structural and molecular studies of the virus particles. Arch. Virol. Suppl. 1998; 14:177202. doi:10.1007/978-3-7091-6823-3_17 
[24] Han MG, Cheetham S, Azevedo M, Thomas C, Saif LJ. Immune responses to bovine norovirus-like particles with various adjuvants and analysis of protection in gnotobiotic calves. Vaccine. 2006; 24(3): 317-326. doi:10.1016/j.vaccine.2005.07.071

[25] Roy P. Nature and duration of protective immunity to bluetongue virus infection. Dev. Biol. (Basel). 2003; 114:169-183. Available from: http://researchonline.lshtm.ac.uk/id/ eprint $/ 15155$

[26] Herzog C, Hartmann K, Künzi V, Kürsteiner O, Mischler R, Lazar H, Glück R. Eleven years of Inflexal V-a virosomal adjuvanted influenza vaccine. Vaccine. 2009; 27(33): 4381-4387. doi:10.1016/j.vaccine.2009.05.029

[27] Monie A, Hung CF, Roden R, Wu TC. Cervarix ${ }^{\mathrm{TM}}$ : a vaccine for the prevention of HPV 16, 18-associated cervical cancer. Biol. Targets Ther. 2008; 2(1): 107-113. Available from: http://www.ncbi.nlm.nih.gov/pmc/articles/PMC2727782/pdf/btt-2-107.pdf

[28] Kash N, Lee MA, Kollipara R, Downing C, Guidry J, Tyring SK. Safety and efficacy data on vaccines and immunization to human papillomavirus. J. Clin. Med. 2015; 4(4): 614633. doi:10.3390/jcm4040614. Review

[29] Lin CY, Lin SJ, Yang YC, Wang DY, Cheng HF, Yeh MK. Biodegradable polymeric microsphere-based vaccines and their applications in infectious diseases. Hum. Vaccines Immunother. 2015; 11(3): 650-656. doi:10.1080/21645515.2015.1009345

[30] Cleland JL. Single-administration vaccines: controlled release technology to mimic repeated immunizations. Trends Biotechnol. 1999; 17(1): 25-29. doi:10.1016/ S0167-7799(98)01272-4

[31] Yeh MK, Coombes AGA, Chen JL, Chiang CH. Japanese encephalitis virus vaccine formulations using PLA lamellar and PLG microparticles. J. Microencapsul. 2002; 19(5): 671-682. doi:10.1080/02652040210142551

[32] Huang SS, Li IH, Hong PD, Yeh MK. Development of Yersinia pestis F1 antigen-loaded microspheres vaccine against plague. Int. J. Nanomed. 2014; 9: 813-822. doi:10.2147/ IJN.S56260

[33] Alonso MJ, Cohen S, Park TG, Gupta RK, Siber GR, Langer R. Determinants of release rate of tetanus vaccine from polyester microspheres. Pharm. Res. 1993; 10(7): 945-953. doi:10.1023/A:1018942118148

[34] Alonso MJ, Gupta RK, Min C, Siber GR, Langer R. Biodegradable microspheres as controlled-release tetanus toxoid delivery systems. Vaccine. 1994; 12(4): 299-306. doi: 10.1016/0264-410X(94)90092-2

[35] Hu CM, Zhang L. Nanotoxoid vaccines. Nano Today. 2014; 9(4): 401-404. doi:10.1016/ j.nantod.2014.06.001 
[36] Makidon PE, Bielinska AV, Nigarekar SS, Janezak KW, Knowlton J, Scott AJ, Mank N, Cao Z, et al. Pre-clinical evaluation of a novel nanoemulsion-based hepatitis B mucosal vaccine. Plos One. 2008; 3(8): e2954. doi:10.1371/journal.pone.0002954

[37] Patel AR, Kulkarni SP, Nandedkar TD, Vavia PR. Evaluation of alkyl polyglucoside (based on C10 fatty alcohol) as alternative surfactant in the preparation of peptide loaded nanoparticles. J. Microencapsul. 2008; 25(8): 531-540. doi: $10.1080 / 02652040802075526$

[38] Kersten G, Hirshberg H. Antigen delivery systems. Expert Rev. Vaccines. 2004; 3(4): 453-462. doi:10.1586/14760584.3.4.453

[39] Belshe RB, Edwards KM, Vesikari T, Black SV, Walker RE, Hultquist M, Kemble G, Connor EM; CAIV-T Comparative Efficacy Study Group. Live attenuated versus inactivated influenza vaccine in infants and young children. N. Engl. J. Med. 2007; 356(7): 685-696. doi:10.1056/NEJMoa065368

[40] Ambrose CS, Luke C, Coelingh K. Current status of live attenuated influenza vaccine in the United States for seasonal and pandemic influenza. Influenza Other Respir. Viruses. 2008; 2(6): 193-202. doi:10.1111/j.1750-2659.2008.00056.x

[41] Jefferson T, Rivetti A, Di Pietrantonj C, Demicheli V, Ferroni E. Vaccines for preventing influenza in healthy children. Cochrane Libr. 8: CD004879, 2012. doi: 10.1002/14651858.CD004879.pub4

[42] Nasal Spray Flu Vaccine in Children 2 through 8 Years Old. CDC. Center for Disease Control and Prevention, US. Retrieved on May 11, 2015. Available from: http:// www.cdc.gov/

[43] Kim YC, Park J-H, Prausnitz MR. Microneedles for drug and vaccine delivery. Adv. Drug Deliv. Rev. 2012; 64(14): 1547-1568. doi:10.1016/j.addr.2012.04.005

[44] van der Maaden K, Luttge R, Vos PJ, Bouwstra J, Kersten G, Ploemen I. Microneedlebased drug and vaccine delivery via nanoporous microneedle arrays. Drug Deliv. Transl. Res. 2015; 5(4): 397-406. doi:10.1007/s13346-015-0238-y

[45] Prausnitz MR, Langer R. Transdermal drug delivery. Nat. Biotechnol. 2008; 26(11): 1261-1268. doi:10.1038/nbt.1504

[46] Donnelly R, Douroumis D. Microneedles for drug and vaccine delivery and patient monitoring. Drug Deliv. Transl. Res. 2015; 5(4): 311-312. doi:10.1007/s13346-015-0250-2

[47] Yeh MK, Chiang CH. Inactive Vibro cholera whole-cell vaccine-loaded biodegradable microparticles: in vitro release and oral vaccination. J. Microencapsul. 2004; 21(1): 91106. doi:10.1080/02652040310001619794 

Chapter 5

\title{
Multiphoton and Fluorescence Lifetime Imaging Microscopy in Studying Nanoparticle Pharmacokinetics in Skin and Liver
}

\author{
Camilla A. Thorling, Amy Holmes, Hauke Studier, \\ David Liu, Xiaowen Liang and Michael S. Roberts
}

Additional information is available at the end of the chapter

http://dx.doi.org/10.5772/63452

\begin{abstract}
The use of nanoparticles has increased in consumer products in recent decades; however, concerns regarding their safety remain. Zinc oxide is used in sunblocking and may generate free radicals in response to UV illumination, leading to DNA damage and an immunological response. With high-resolution, high-contrast imaging in biological tissue, multiphoton microscopy is able to separate nanoparticles signals from endogenous fluorophores. It has been proven to be very useful in imaging penetration of zinc oxide nanoparticles in skin and in combination with fluorescence lifetime imaging microscopy study cellular function as well. This chapter aims to review the use of these imaging techniques in studying the uptake and distribution of nanoparticles in skin and liver. Due to the questionable clinical use and possible toxicity of nanoparticles, it is important to study their pharmacokinetics. Some nanomaterials have been identified as relatively toxic to humans and a few metal nanoparticles have been reported to penetrate and be detected in blood. Multiphoton microscopy has high resolution and is able to visualize nanoparticles, due to their optical properties, in vivo. The addition of fluorescence lifetime imaging makes it possible to measure the physiochemical environment, with outputs that can be statistically analyzed, posing an advantage over fluorescence intensity imaging only.
\end{abstract}

Keywords: multiphoton microscopy, fluorescence lifetime imaging microscopy, nanoparticles, skin, liver 


\section{Introduction}

Nanoparticles (NPs) are particles ranging from 1 to $100 \mathrm{~nm}$ in size and are a promising pharmaceutical tool for drug delivery and functionalized cosmetic products like sunscreens. There are growing public and regulatory concerns of topical application of NPs, due to the increasing manufacturing of NPs in commercial products as well as continuing discovery of new applications. NPs are available in several different shapes, such as sphere, rod, cylinder, and cube. Furthermore, they can be soft or hard, aggregated or dispersed. NPs present in commercial products are often made from metals such as gold and silver or metal oxides like zinc oxide and titanium oxide, but also include quantum dots (QDs), carbon nanotubes, fullerenes, and lipid-based materials. From anenvironmental and occupational health and safety point of view, it is important to investigate NPs interaction with organs following unintended exposure. On the other hand, it is very necessary to study their properties in active drug delivery and clearance without adverse effects for therapeutic and cosmetic applications. Properties, such as particle size and shape, surface charge, $\mathrm{pH}$, formulation, are important factors in determining the penetration of NPs in the skin. Toxicity of NPs is mainly of concern for the smaller particles. The skin may be more susceptible to NPs penetration if it is diseased, hot, damaged, inflamed, hydrated, dry, or moisturized. Positively charged NPs are most preferred for skin penetration [1].

Determining the presence or absence as well as concentration of NPs in biological tissue has been enabled by multiphoton microscopy (MPM), especially in investigating interactions of NPs with human skin [2].

Most knowledge in the biological world has been gathered from studying images [3]. Thus, a growing interest for live-tissue imaging has evolved. In conventional confocal microscopy, the intensity from the beam is approximately uniform above and below the focal plane, which results in the specimen generating fluorescence out of the focal plane that is rejected by the pinhole. This leads to the specimen being subjected to photobleaching and photodamage, affecting image quality, and tissue health [4]. MPM avoids this because a much smaller area of the specimen is being stimulated by the excitation light source and no out of focus light is generated, which leads to photobleaching being restricted to the focal point only [4, 5]. At an excitation wavelength in the near infrared range (NIR, $700-1000 \mathrm{~nm}$ ), the photon penetration depth of the incident light is maximized, and the tissue scattering and absorption are minimized [6, 7].

In confocal microscopy (single photon), fluorescence occurs when a photon is absorbed by a fluorescent molecule and raises an electron to an excited energy state (Figure 1). When the electron returns to the ground state, it converts the absorbed energy to heat, by transferring the energy to another molecule emitting a lower energy photon $[8,9]$, producing an image. In MPM, two (or more) photons of the same energy from a pulsed laser (usually femtosecond laser) interact with a molecule to produce excitation equivalent to the absorption of a single photon possessing twice (or multiple) the energy (Figure 1). If the excited molecule is fluorescent, it can emit a single photon [8-10]. 


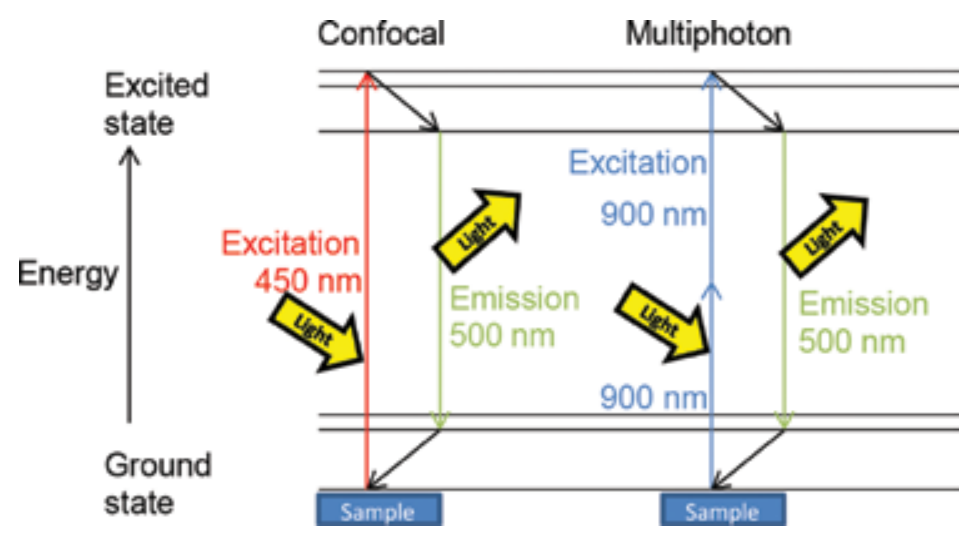

Figure 1. Jablonski energy diagram (adapted from Refs. [8-11]). Imagine a fluorescent molecule with single absorption and emission at 450 and $500 \mathrm{~nm}$, respectively. In order to excite electron(s) from this molecule, light from a laser is needed. The electron(s) is then raised into a higher excited state, where it decays rapidly into the ground vibrational state within the electronically excited state. From there the electron can return to the electronically ground state by emitting light, which can yield in fluorescence. The emitted light has lower energy (higher wavelength) than the absorbed light. The emission spectrum of fluorescence excited by MPM is usually similar to the one excited with single photon excitation. To excite the molecule with two-photon, two photons of equal wavelengths, which together meet the energy gap between the ground and excited states, are needed (here $900 \mathrm{~nm}$ ).

An important application of MPM is to image the physiology, morphology, and cell-cell interactions in intact tissue of live animals with high resolution. However, one limitation is that it cannot quantitatively study cellular function on a molecular level [12]. MPM in combination with fluorescence lifetime imaging microscopy (FLIM), however, can identify fluorophores with overlapping spectral properties. Furthermore, it enables insights into their biological function by being sensitive to the binding site and the environmental surrounding [13]. The fluorescence lifetime is proportional to the reciprocal Einstein coefficient of the spontaneous emission, that is, to the sum of the rate constants of all possible return paths for the electron from the excited state to the ground state that is not stimulated emission [14]. This is determined by both intramolecular and intermolecular events [4]. FLIM has been described as a direct approach to monitor energy transfers between fluorophore and the environment, for example, DNA binding, which change the fluorescence lifetime of that fluorophore [15]. FLIM is particularly important when identifying fluorophores with overlapping spectral properties [13]. One application for FLIM in liver imaging is to study levels of the autofluorescent $\mathrm{NADH}$, as a direct measure for the redox state as a metabolic marker of the cells [8].

The principle of FLIM is illustrated in Figure 2. Imagine a sample with two regions, each with equal intensity, but two different fluorescence lifetimes, one shorter $(\tau 1)$ and one longer $(\tau 2)$. $\tau 2$ could be due to binding to other molecules, change in $\mathrm{pH}$, cation concentration, oxygen concentration, or polarity. The intensity image itself can not reveal these environmental differences (Figure 2a), but the FLIM image can (Figure $\mathbf{2 b}$ and $\mathbf{c}$ ). The fluorescence lifetimes within a FLIM image can be presented on a grey (Figure $\mathbf{2 b}$ ) or colour scale (Figure $\mathbf{2 c}$ ) or as three-dimensional (3D) surface where the height represents the local decay times (Figure 2d) [16]. 

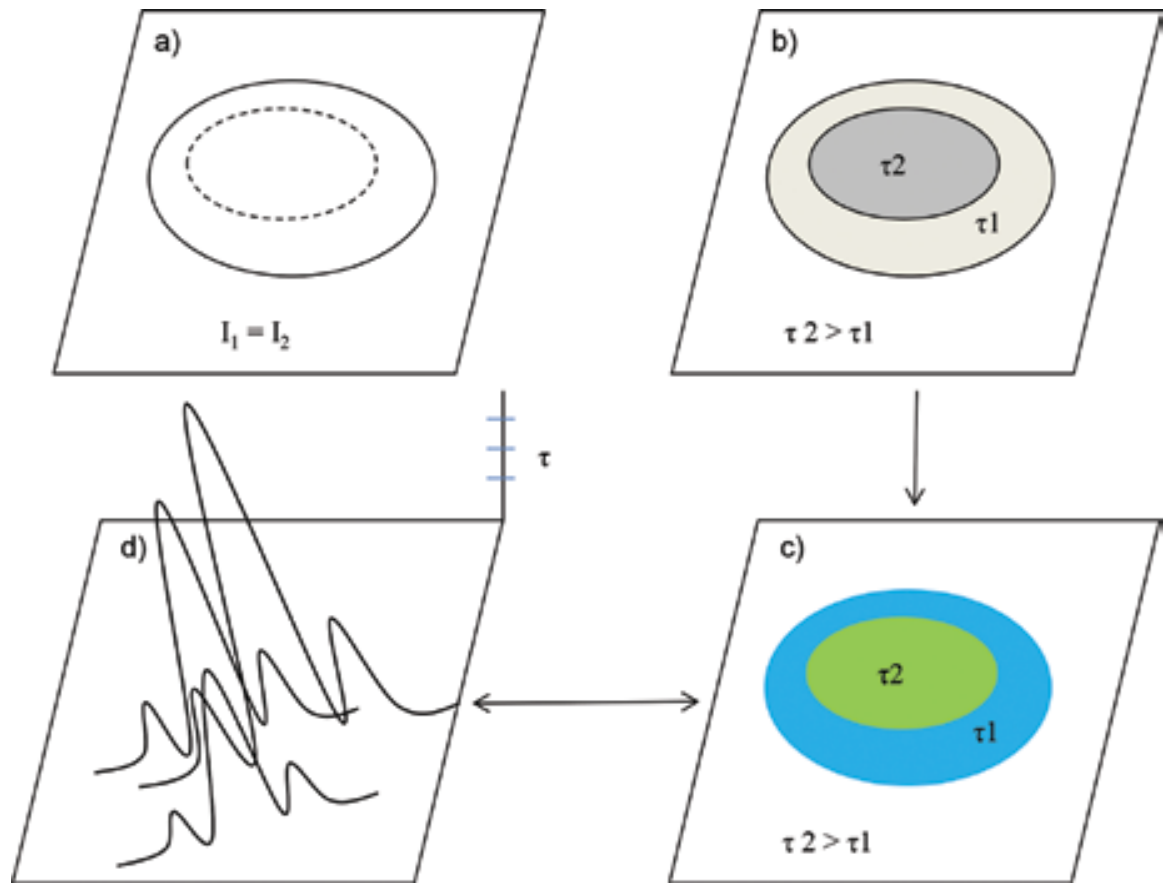

Figure 2. Principles of fluorescence lifetime imaging (FLIM). (a) Intensity (I) cannot reveal any difference in the surrounding environment; (b) FLIM enables distinguishing the two components with different fluorescence lifetime and can give insights into biological function (grey scale represents fluorescence lifetimes, where $\tau 2>\tau 1$ ). (c) Fluorescence lifetimes can also be colour coded or; (d) displayed as third coordinate, resulting in a 3D image (adapted from Ref. [Lakowitz et al., Fluorescence lifetime imaging of free and protein-bound NADH. Proceedings of the National Academy of Sciences 1992 89(4): 1271-1275).

MPM can be used to image fluorescent and sometimes even nonfluorescent NPs in living cells, tissue, and organs in vivo. Nonfluorescent NPs can have nonlinear optical properties so that they can become visible for MPM (e.g., zinc oxide ( $\mathrm{ZnO}) \mathrm{NPs}$, gold (Au) NPs, and silver (Ag) NPs) [17]. One physical phenomenon is second harmonic generation (SHG). It is based on elastic scattering. The wavelength of the SHG signal is at the double frequency of the incident excitation light of the femtosecond laser. The optical response is quasi-instantaneous and therefore can easily be identified by FLIM. A second process is the so-called localized surface plasmon resonance (LSPR) [18]. The LSPR signal can be tailored by modifying the size or the shape of the NPs. This signal can be very broadband (inelastic phenomenon) and strong but is also characterized by a very rapid optical response in the femtosecond range so that it also can easily be identified by FLIM technique [19].

MPM has proven to be very useful in imaging the uptake of metal NPs, such as ZnO-NPs, AuNPs and Ag-NPs in the skin. Additionally, NAD(P)H (NADH+NADPH) can be imaged simultaneously without the need for additional dyes using FLIM. This can help to understand how NPs affect the viable skin condition. The additional time-resolved measurements with FLIM can be used to differentiate NP signals from endogenous tissue [2]. This chapter aims to critically review the use of these imaging techniques in studying the penetration of NPs and 
quantum dots (QDs) into skin, how to facilitate intentional uptake, as well as the uptake and distribution in the liver.

\section{Topical application of zinc oxide nanoparticles}

Zinc oxide NPs (ZnO NPs) are incorporated into a plethora of commercially available formulations including sunscreens, cosmetics such as mineral-based make-up, and nappy rash ointments. Though ZnO NPs are considered to be safe after topical application [20], there have been concerns raised about the lack of assessment of toxicity under "in-use" conditions [21]. $\mathrm{ZnO}$ NPs are transparent and affords the viable epidermis broad spectrum protection from harmful UV radiation. Irradiation of $\mathrm{ZnO}$ NPs, however, can result in the production of reactive oxygen species (ROS) that are harmful if exposed to the keratinocytes within the viable epidermis [22]. It is therefore of importance to delineate the deposition of $\mathrm{ZnO} N P$ s after topical application to comprehensively assessing whether ZnO NPs can penetrate the stratum corneum and reach the viable epidermis. A ZnO NP suspension was applied to human skin in vivo at $10 \% \mathrm{wt}$ for 6 hours and then occluded to simulate clothing covering the skin with the applied sunscreen. It was found that occlusion, and thus, skin hydration does not aid the penetration of ZnO NPs into human skin and no localized toxicity was observed within the viable epidermis using metabolic lifetime imaging [23]. In this study, the photoluminescence of $\mathrm{ZnO}$ NPs was spectrally resolved from that of the endogenous fluorophores, and thus, the deposition was mapped after application to the volunteers' forearms. The fluorescence lifetime amplitude, $\alpha_{1} \%$, of ZnO NPs (multiphoton-excited photoluminescence) was found to be distinct from that of the autofluorescence. This enabled the authors' to map the distribution of ZnO NPs after application to viable skin in vivo. Further, using MPM-FLIM, the free-to-bound ratio of endogenous $\mathrm{NAD}(\mathrm{P}) \mathrm{H}$ was determined to elucidate any effect $\mathrm{ZnO} \mathrm{NP}$ application had on the redox state of the viable epidermis.

Other studies conducted include the in vivo application of ZnO NPs with massage and arm flexing in which the conclusions were that again no ZnO NP penetration as observed with the exception of $\mathrm{ZnO}$ NPs within the sebaceous gland of one volunteer and no localized toxicity was observed by metabolic imaging [23]. An in vivo study was also conducted to assess any $\mathrm{ZnO}$ penetration after repeated exposure [25] as the cancer council advises the public to reapply sunscreen every 2 hours [24]. In this study, once again no $\mathrm{ZnO} \mathrm{NP}$ penetration or localized toxicity within the viable epidermis was observed even after repeated liberal application to volunteers' forearm [25]. Lin et al. applied ZnO NPs to intact human skin and also skin that was tape-stripped (20×), or had psoriatic and atopic dermatitis lesions. The study showed that the $\mathrm{ZnO} N \mathrm{~N}$ photoluminescence signal was most intense at the skin surface after application to impaired lesioned skin compared to intact skin in vivo. In this study, no penetration of $\mathrm{ZnO} N P$ was observed within the viable epidermis after application of $\mathrm{ZnO} N P s$ in caprylic capric triglyceride (CCT) [26].

As NPs have a high surface-to-volume ratio, the reactivity and dissolution of constituent ions increases with decreasing diameter of the NP. Zinc ion release and percutaneous absorption has been observed after topical ZnO NP application to human skin both in vivo [27-29] and in 
vitro [30]. Further, NPs are known to accumulate within the hair follicles and undulations of human skin for up to 10 days [31] whereby they may act as a reservoir undergoing dissolution into constituent solubilized species within these reduced environments. To increase stability, reduce dissolution and production of ROS, coated ZnO NPs have been employed within formulations. Leite-Silva et al. [23] investigated the deposition of ZnO NPs after application to in vivo human skin within various formulations. MPM-FLIM was used once again to simultaneously map ZnO NP deposition whilst also determining the $\mathrm{NAD}(\mathrm{P}) \mathrm{H}$ free-to-bound ratio of the underlying viable epidermis after application of both coated and uncoated $\mathrm{ZnO}$ NPs in (a) CCT suspension, (b) a gel formulation, (c) oil-in-water emulsion, and (d) water-inoil emulsion. The study reported the deposition of the ZnO NPs only within the superficial layers of the stratum corneum and within the furrows as reported previously after application in a water-in-oil emulsion (Roberts MS, Roberts MJ, Robertson TA, Sanchez W, Thorling CA, Zou Y, Zhao X, Becker W and Zvyagin AV. In vitro and in vivo imaging of xenobiotic transport in human skin and in the rat liver. Journal of Biophotonics 1(6): 478-493, 2008). The exception was minimal $\mathrm{ZnO} N P$ penetration was observed within isolated areas in parallel to the furrow within the stratum granulosum after application of coated $\mathrm{ZnO} N P$ s in a water-in-oil emulsion observed in Figure 3 [23]. This, however, did not result in a change in redox state within the stratum granulosum; therefore, the penetrated ZnO NPs did not cause toxicity [23].

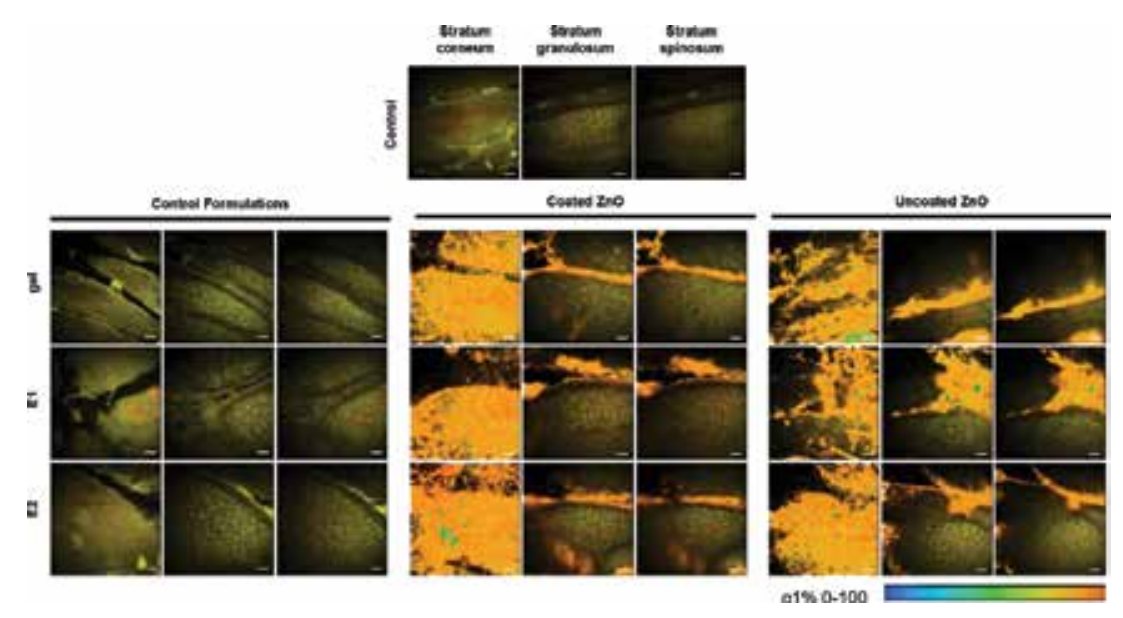

Figure 3. Pseudocoloured MPM-FLIM images of human skin treated with ZnO NPs in vivo. ZnO NPs were dispersed in three types of formulations, gel, emulsion E1 o/w, and emulsion E2 w/o in coated or uncoated form. Control images do not contain any NPs. The green colour corresponds to skin autofluorescence $\left(\alpha_{1} \% 45-85\right)$, whereas the orange-red colour corresponds to ZnO-NPs photoluminescence $\left(\alpha_{1} \% 90-100\right)$. Scale bar is $20 \mu \mathrm{m}$. The colour scale bar represents $\alpha_{1} \% 0-100$.

\section{Topical application of gold nanoparticles}

Gold NPs (Au NPs) are being increasingly investigated for topical drug delivery due to their monodispersed controllable size, the ability to functionalize the surface of the Au NPs and due 
to their low reactivity potential. Recently, Au NPs have been investigated as a drug delivery platform in the treatment of psoriasis [32] and as contrast agents in skin cancer imaging [33]. The Au NP (6-15 nm in diameter) penetration study showed $15 \mathrm{~nm}$ AuNP in aqueous solution was also observed by MPM-FLIM to aggregate within furrows of skin after treated on excised viable skin sample and also showed penetration of $6 \mathrm{~nm}$ Au NP suspended in toluene within the stratum spinosum of the viable epidermis [34]. No change in the redox state of the viable epidermis was observed when Au NPs were applied to the skin in an aqueous vehicle though a change in redox state was observed when Au NPs were applied in toluene [34]. The authors' further investigated the penetration of Au NPs into skin using dimethyl sulfoxide (DMSO), a known chemical penetration enhancer, and found that DMSO enhanced the penetration of $\mathrm{Au}$ NPs into skin [35]. A study also showed that Au NP skin penetration was found to be size dependent with $6 \mathrm{~nm}$ Au NPs penetrating to higher degree than $15 \mathrm{~nm}$ Au NPs [35]. Fernandes et al. found that the skin penetration on Au NPs could be tailored depending on the surface chemistry and shape of the NP [36]. Larese Filon et al. also concluded that Au NPs can penetrate into both intact and impaired human skin in vitro [37]. Conversely, Lin et al. found no

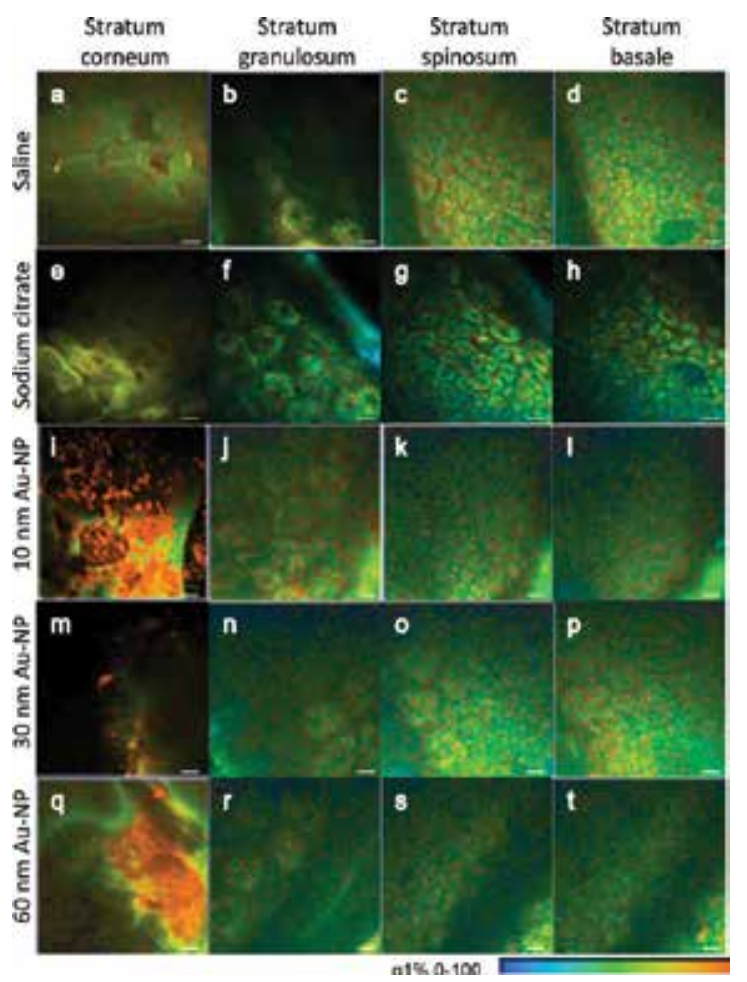

Figure 4. En face MPM-FLIM images of treated human viable skin. FLIM images showing the different layers of excised full-thickness skin (stratum corneum, granulosum, spinosum, and basale) after 24-hour treatment with Au NPs suspended in sodium citrate. Images are pseudocoloured based on $\alpha_{1} \%$ signal (skin autofluorescence $0-95 \%$ and AuNPs 95-100\% signal). No Au NP signal was detected in the saline control (a)-(d) and detected in the sodium citrate control (e)-(h). The AuNP-treated groups, $10 \mathrm{~nm}(\mathrm{i})-(\mathrm{l}), 30 \mathrm{~nm}(\mathrm{~m})-(\mathrm{p})$, and 60nm (q)-(t), resulted in signal throughout the stratum corneum. Scale bar indicates $20 \mu \mathrm{m}$. Colour bar, blue to red indicates $\alpha_{1} \% 0-100 \%$. 
penetration of Au NPs after topical application to human skin in vivo (as shown in Figure 4). In this study, Au NPs of increasing diameter 10, 30, and $60 \mathrm{~nm}$ were applied to viable ex vivo human skin and MPM-FLIM was used to assess any percutaneous absorption of Au NPs. No penetration of $\mathrm{Au}$ NPs was observed past the stratum corneum and no change in the redox state of the viable epidermis was observed indicating no localized toxicity [38]. The scientific community has yet to reach a consensus on whether inorganic NPs can penetrate human skin as exhibited by conflicting results within the literature.

\section{Topical application of other nanoparticles}

SECosomes are flexible nanovesicles constituted from surfactant, ethanol, and cholesterol, hence the name SECosomes. MPM-FLIM was used to observe the penetration of SECosomes into viable ex vivo human skin as deep at $40 \mu \mathrm{m}$ within the viable epidermis, which resulted in a shift of lifetime for NAD $(\mathrm{P}) \mathrm{H}$ [39]. Silver NPs (Ag NPs) are found within a wide variety of personal care products including deodorants', antibacterial under garments, and wound dressings. To determine whether Ag NPs penetrate human skin in vivo, Ag NPs were applied to intact or tape stripped skin. No statistically significant difference in metabolic rate measuring the inverse ratio of free-to-bound $\mathrm{NAD}(\mathrm{P}) \mathrm{H}\left(\alpha_{1} / \alpha_{2}\right)$ was observed within the viable epidermis [40]. In a more recent study, the shape dependence of Ag NPs and their penetration into human skin was determined on ex vivo human skin [19]. No Ag NPs either cube, sphere, or truncated plates were observed within the viable epidermis in intact skin but Ag NP plates were found within the wound bed of burned human skin. In this study, Ag NPs penetration into both intact and burned human skin was assessed through detection of both the second harmonic generation (SHG) signal and the localized surface plasmon resonance (LSPR) of the NPs. Using MPM-FLIM, the detected nonlinear optical characteristics (SHG and LSPR) found that the Ag NPs accumulated within the furrows of the skin, and in particular, the truncated Ag NP plates showed great substantivity within the superficial layers of the stratum corneum but that no penetration into the viable epidermis was observed for intact skin [19]. The authors' also observed that Ag NP spheres accumulated within and around the hair follicle shafts after topical application. This was also supported by the work of Zhu et al. [41] as they too did not observe any penetration of Ag NPs into the viable epidermis of intact porcine skin but did image Ag NPs within the hair follicles using MPM-FLIM and Raman scattering microscopy.

QDs are 2-10 nm fluorescent semiconductor nanomaterials with a larger excitor Bohr radius than the NP radius, making these nanomaterials to undergo quantum confinement. As a result, QDs have characteristic excitation states and larger bandgap values than bulk materials. Emission wavelengths is dependent on size, bandgap increases as the radius decreases (i.e., for smaller QDs). They have greater resistance to photobleaching and have higher quantum yield than conventional fluorophores [42]. Here, they characterized water-soluble cadmium selenide-zinc sulphide quantum dots for MPM imaging in live animals. They visualized QDs dynamically through mice skin in capillaries hundreds of micrometres deep [43].

The advantages that MPM-FLIM provides over other imaging techniques as outlined in the Introduction section have enabled numerous researchers to map the deposition and potential 
percutaneous absorption of a wide variety of NPs into skin. Further elucidation is required, however, to determine whether NPs can penetrate human skin due to conflicting conclusions within the literature and with the nanotechnology sector rapidly increasing the risk of dermal exposure to NPs will increase.

\subsection{How do we improve penetration of nanoparticles for drug delivery?}

The penetration of NPs can be improved by penetration enhancers, such as oleic acid, urea, sodium lauryl sulphate, polysorbate, and DMSO. MPM was used to visualize the penetration of a fluorescent NP with the assistance of oleic acid. The results showed that oleic acid was effective in facilitating transdermal delivery of NPs [44]. Au NPs penetration was studied with the chemical enhancers, urea, sodium lauryl sulphate, polysorbate, and DMSO, and it was evident that the penetration was induced by DMSO [35]. Similarly, chemical enhancers can increase penetration of $\mathrm{ZnO}$ NPs, by increasing lipid fluidity or by extracting noncovalently bound amphiphilic lipid in the stratum corneum [26].

Another way of increasing penetration of NPs is by using microneedles, which can create pores in the stratum corneum layer, which enables delivery of NPs to the deeper layers of the skin. Gantrez R_AN-139 microneedle successfully did this [45]. Two-photon polymerization of an acrylate-based polymer was used to fabricate microneedle devices for transdermal drug delivery and MPM visualized QDs penetration in porcine skin by a microneedle device [42].

\section{Nanoparticles disposition in the liver}

NPs are increasingly being used for the detection and treatment of human diseases, in applications such as drug and gene delivery, imaging, and diagnostics [46, 47]. However their short in vivo half-lives limit their clinical utility and can lead to hepatotoxicity [46]. It is important to monitor the biodistribution of NPs after intravenous administration in order to evaluate their efficacy and possible side effects. The physiochemical properties of NPs, such as size, shape, surface charge are important in determining the biodistribution [2-5]. For example, hydrophobic NPs are rapidly removed by reticuloendothelial system (RES), mainly by the liver and spleen [46]. Additionally, intravital imaging showed that positively charged NPs where taken up by the hepatocytes (liver cells), whereas negatively charged NPs were found to be taken up by Kupffer cells, liver macrophages, instead [46]. The positively charged NPs have the potential to be cleared via hepatobiliary excretion; however, the negatively charged NPs portends significant hepatotoxicity due to their accumulation in Kupffer cells [46]. If NPs are taken up by hepatocytes, this would be through receptor-mediated endocytotic processes [48]. This is in contrast to smaller molecules, such as RH123 and fluorescein, which are taken up by diffusion and transporter proteins in the liver membranes [49].

In a recent study, a multimodal nonlinear label-free imaging technique to pinpoint polymeric NPs within the intestine and liver was done. Encapsulation of active substances within nanoscale particles can enhance bioavailability and biocompatibility, resulting in targeted drug delivery, solubility, and bioactivity and reducing toxicity. They used MPM to show 
cellular structures, SHG to visualize collagen and CARS to generate chemical-specific images of polymer-based NPs with contrast derived from the molecular vibration of carbon-deuterium bonds within the polymer's palmitic acid chains. These modalities combined give accurate location of the NPs in relation to the cellular structure in the liver, gall bladder, and intestine [50].

The use of MPM to visualize the delivery of Cy5-siRNA with lipid NPs vehicles to hepatocytes was shown by Chen et al. They analysed the diffusion of Cy5-siRNA into the hepatocytes by computation of the percentage signal strength in the region of interest over time [51].

Quantum dots (QDs) are nanomaterials recognized as promising diagnostic and imaging agents. QDs are the most effective semiconductor materials for applications in the field of bioimaging, since they have unique optical and photophysical properties, with high quantum yield and strong fluorescence at both visible and NIR wavelengths. QDs are also stable and retain their fluorescence for a long period of time. QDs have been found to be retained in the organs of the RES for over 22 days after injection, suggesting that they are consumed by mononuclear phagocytes [52]. QDs are particularly well suited for the detection of low abundance antigens, such as hepatitis $\mathrm{C}$ virus (HCV), because they are less prone to photobleaching. MPM has been used in combination with QDs to visualize the distribution of HCVinfected cells within the liver [53].

In a previous study, our group applied MPM-FLIM to investigate the in vivo subcellular, spatiotemporal disposition of negatively charged QDs in rat liver. The cellular behaviour of QDs was found to be very different from traditional fluorescent molecules, rhodamine 123 (RH123) and fluorescein (Figure 5). Results showed that QDs were quickly detected in the liver sinusoids immediately after injection and were not taken up by hepatocytes, but the fluorescence stayed in the sinusoids and started to decrease after about $60 \mathrm{~min}$. The QDs were found to be taken up by Kupffer cells and liver sinusoidal endothelial cells (LSECs).

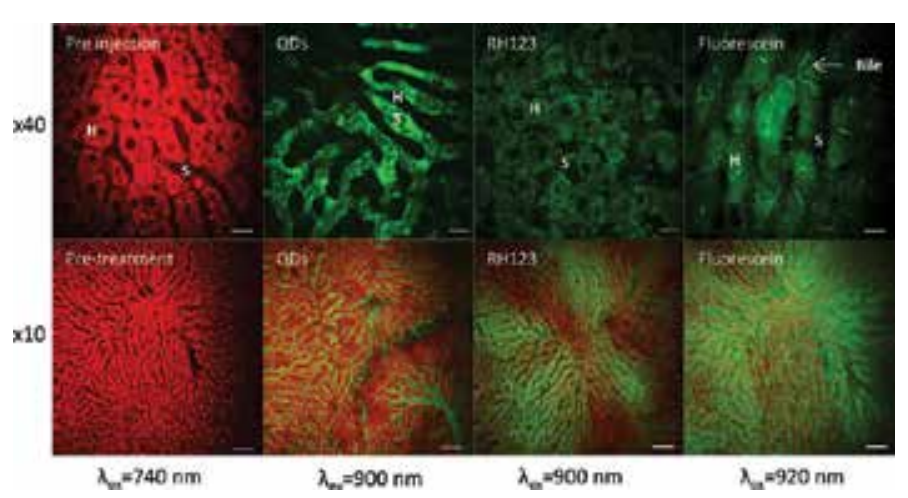

Figure 5. False-coloured images of QDs, RH123, and fluorescein distribution in the rat liver at 5 min after injection (adapted from Ref. [49]) using high $(\times 40)$ and low $(\times 10)$ magnification objectives. Red colour represents autofluorescence of the liver excited at $740 \mathrm{~nm}$. Green colour represents the fluorophores, QDs, RH123, and fluorescein, excited at 900 and $920 \mathrm{~nm}$. Scale bar is $20 \mu \mathrm{m}, \mathrm{H}$ = hepatocyte, $\mathrm{S}=$ sinusoid, arrow points to bile ducts. 
The distribution of these QDs was similar to the distribution of rhodamine B isothiocyanate/ dextran 7000, which is a fluorescent marker for labelling the sinusoids of the liver. Additionally, this is in accordance to another study where they found no uptake of negatively charged fluorescein isothiocyanate-labelled mesoporous silica NPs [16]. Both RH123 and fluorescein are excreted through the bile, and hence, the fluorescence signal in the liver declined rapidly, but since these QDs were not excreted through the bile, the fluorescence declined much slower. The fluorescence lifetime of the sinusoids increased after QDs injection, which was due to the long lifetime of QD [49].

Fast and reliable ratiometric FLIM (rmFLIM) approach is described to analyse the distribution of protein-ligand complexes in the cellular context. In combination with Forster resonance energy transfer (FRET), FLIM can be used to map protein-protein interactions on the nanometer-scale in living cells. Organic fluorophores label the ligands in the case of membrane receptors or intracellular proteins that bind extracellular ligands. Imaging based on fluorescence intensity is often used to localize cellular sites where the ligand binds to its cognate receptor, but these techniques may be biased by unspecific binding and distribution of the ligand in various subcellular compartments. FLIM is sensitive to the physiochemical environment, posing an advantage over fluorescence intensity measurements. In this study, they show a fast and reliable FLIM-based method for the localization of target molecules and their discrimination against the fluorescent background of cell membranes and tissue. FRET relies on the shortening of the donor lifetime due to the less energy transfer of radiation to the acceptor. Physiochemical environment can also affect the fluorescence lifetime, for example, quenching. The shortening of fluorescein's fluorescence lifetime when it binds to Cys316 in the opsin molecule results from additional dynamic interaction of the bound dye with the surrounding protein matrix, which functions as a quencher. A ratiometric FLIM (rmFLIM) relies on the specific multiexponential lifetime signature of a protein-ligand complex that is unique compared to the fluorescence lifetime distribution of the background. Only one fluorophore is needed. rmFLIM was used to analyse the fate of a polymer-based nanocarrier for drug delivery in the metabolic clearance process. They used a polyanionic, dendritic polyglycerolsulfate (dPGS) labelled with a fluorescent indocarbocyanine dye (ICC, spectrally analogue to $\mathrm{Cy} 3$ ). In solution, this nanocarrier had 2 fluorescence lifetimes of 0.27 and $1.1 \mathrm{~ns}$. Paraffin-embedded liver section from rats, previously injected with dPGS-ICC, was used for FLIM measurements [54].

\section{Conclusions and future directions}

In this chapter, we review the use of MPM and FLIM to visualize the disposition of NPs and QDs in the skin and liver. Nanotechnology and its applications in dermatology and drug delivery have received a growing interest over the past years. Increased applications of NPs have led to an enhanced unintentional skin exposure of NPs and it is therefore important to study when and how NPs penetrate skin. Some conclusions of NP penetrations into skin are as follows: (1) The SC forms an effective barrier to NPs penetration, but smaller-sized NPs are more likely to penetrate. (2) Hair follicles are an important collection site for NPs, and 
increasingly so when the skin is massaged or flexed. (3) The surface charge and formulation of NPs can affect its penetration.

Negatively charged QDs were found to distribute immediately after injection in the sinusoids of mice livers and accumulated in Kupffer cells and LSECs. Positively charged NPs, however, can be taken up by hepatocytes and subsequently be subjected to biliary excretion. It is important to know where NPs are localized after intravenous injection as these cells are emerging as significant targets for therapies in many liver diseases.

\section{Author details}

Camilla A. Thorling ${ }^{1 *}$, Amy Holmes ${ }^{2}$, Hauke Studier ${ }^{2}$, David Liu ${ }^{1}$, Xiaowen Liang ${ }^{1}$ and Michael S. Roberts ${ }^{1,2}$

*Address all correspondence to: c.thorling@uq.edu.au

1 Therapeutics Research Centre, Translational Research Institute, School of Medicine, The University of Queensland, Brisbane, Australia

2 School of Pharmacy and Medical Sciences, The University of South Australia, Adelaide, Australia

\section{References}

[1] Liang XW, Xu ZP, Grice J, Zvyagin AV, Roberts MS, Liu X. Penetration of nanoparticles into human skin. Current Pharmaceutical Design. 2013;19(35):6353-66.

[2] Prow TW, Monteiro-Riviere NA, Inman AO, Grice JE, Chen X, Zhao X, et al. Quantum dot penetration into viable human skin. Nanotoxicology. 2012;6(2):173-85.

[3] Eliceiri KW, Rueden C. Tools for visualizing multidimensional images from living specimens. Photochemistry and Photobiology. 2005;81(5):1116-22.

[4] Emptage NJ. Fluorescent imaging in living systems. Current Opinion in Pharmacology. 2001;1(5):521-5.

[5] Stutzmann GE, Parker I. Dynamic multiphoton imaging: a live view from cells to systems. Physiology. 2005;20(1):15-21.

[6] Masters BR, So PT. Confocal microscopy and multi-photon excitation microscopy of human skin in vivo. Optics Express. 2001;8(1):2-10.

[7] Hoover EE, Squier JA. Advances in multiphoton microscopy technology. Nature Photonics. 2013;7(2):93-101. 
[8] Roberts MS, Dancik Y, Prow TW, Thorling CA, Lin LL, Grice JE, et al. Non-invasive imaging of skin physiology and percutaneous penetration using fluorescence spectral and lifetime imaging with multiphoton and confocal microscopy. European Journal of Pharmaceutics and Biopharmaceutics. 2011;77(3):469-88.

[9] Oheim M, Michael DJ, Geisbauer M, Madsen D, Chow RH. Principles of two-photon excitation fluorescence microscopy and other nonlinear imaging approaches. Advanced Drug Delivery Reviews. 2006;58(7):788-808.

[10] Helmchen F, Denk W. Deep tissue two-photon microscopy. Nature Methods. 2005;2(12):932-40.

[11] Campagnola PJ, Clark HA, Mohler WA, Lewis A, Loew LM. Second-harmonic imaging microscopy of living cells. Journal of Biomedical Optics. 2001;6(3):277-86.

[12] Niesner RA, Andresen V, Gunzer M. Intravital two-photon microscopy: focus on speed and time resolved imaging modalities. Immunological Reviews. 2008;221(1):7-25.

[13] Yan L, Rueden CT, White JG, Eliceiri KW. Applications of combined spectral lifetime microscopy for biology. Biotechniques. 2006;41(3):249.

[14] Becker W. Fluorescence lifetime imaging-techniques and applications. Journal of Microscopy. 2012;247(2):119-36.

[15] Errington RJ, Ameer-Beg S, Vojnovic B, Patterson LH, Zloh M, Smith PJ. Advanced microscopy solutions for monitoring the kinetics and dynamics of drug-DNA targeting in living cells. Advanced Drug Delivery Reviews. 2005;57(1):153-67.

[16] Lakowicz JR, Szmacinski H, Nowaczyk K, Johnson ML. Fluorescence lifetime imaging of free and protein-bound NADH. Proceedings of the National Academy of Sciences. 1992;89(4):1271-5.

[17] Podlipensky A, Lange J, Seifert G, Graener H, Cravetchi I. Second-harmonic generation from ellipsoidal silver nanoparticles embedded in silica glass. Optics Letters. 2003;28(9):716-8.

[18] Mock J, Barbic M, Smith D, Schultz D, Schultz S. Shape effects in plasmon resonance of individual colloidal silver nanoparticles. The Journal of Chemical Physics. 2002;116(15): 6755-9.

[19] Holmes A M, Lim J, Studier H, S RM. Varying the morphology of silver nanoparticles results in differential toxicity against micro?organisms, human keratinocytes and affects skin deposition. 2016 (unpublished data).

[20] Scientific Committee on Consumer Safety OPINION ON Zinc oxide (Nano Form); 2012, SCCS/1489/12. Available from: http://ec.europa.eu/health/scientific_committees/ consumer_safety/docs/sccs_o_137.pdf 
[21] Miller G, Sales L. Nano ingredients in sunscreen: the need to regulation. Friends of the Earth 2012. Available from: http://emergingtech.foe.org.au/resources/nano-ingredients-in-sunscreen-the-need-for-regulation/

[22] Graf BW, Chaney EJ, Marjanovic M, De Lisio M, Valero MC, Boppart MD, et al. In vivo imaging of immune cell dynamics in skin in response to zinc-oxide nanoparticle exposure. Biomedical Optics Express. 2013;4(10):1817-28.

[23] Leite-Silva VR, Le Lamer M, Sanchez WY, Liu DC, Sanchez WH, Morrow I, et al. The effect of formulation on the penetration of coated and uncoated zinc oxide nanoparticles into the viable epidermis of human skin in vivo. European Journal of Pharmaceutics and Biopharmaceutics. 2013;84(2):297-308.

[24] Cancer Council Australia. Available from: www.cancer.org.au.

[25] Mohammed Y, Sanchez W, Haridass IN, Pilorget C, Penloup AL, Studier H, Chandrasekaran $\mathrm{N}$, et al. Does repeated topical application of zinc oxide nanoparticles over 5 days result in penetration into human skin in vivo? (unpublished work) 2016.

[26] Lin LL, Grice JE, Butler MK, Zvyagin AV, Becker W, Robertson TA, et al. Timecorrelated single photon counting for simultaneous monitoring of zinc oxide nanoparticles and NAD (P) H in intact and barrier-disrupted volunteer skin. Pharmaceutical Research. 2011;28(11):2920-30.

[27] Gulson B, Wong H, Korsch M, Gomez L, Casey P, McCall M, et al. Comparison of dermal absorption of zinc from different sunscreen formulations and differing UV exposure based on stable isotope tracing. Science of the Total Environment. 2012;420:313-8.

[28] Gulson B, McCall M, Korsch M, Gomez L, Casey P, Oytam Y, et al. Small amounts of zinc from zinc oxide particles in sunscreens applied outdoors are absorbed through human skin. Toxicological Sciences. 2010, 118, 140-149.

[29] Ågren M. Percutaneous absorption of zinc from zinc oxide applied topically to intact skin in man. Dermatology. 1990;180(1):36-9.

[30] Holmes AM, Song Z, Moghimi HR, Roberts MS. Relative penetration of zinc oxide and zinc ions into human skin after application of different zinc oxide formulations. ACS Nano, 2016, 10 (2), pp 1810-1819.

[31] Lademann J, Richter H, Teichmann A, Otberg N, Blume-Peytavi U, Luengo J, et al. Nanoparticles - an efficient carrier for drug delivery into the hair follicles. European Journal of Pharmaceutics and Biopharmaceutics. 2007;66(2):159-64.

[32] Bessar H, Venditti I, Fratoddi I, Benassi L, Vaschieri C, Azzoni P, et al. Functionalized gold nanoparticles for topical delivery of Methotrexate for the possible treatment of psoriasis. Colloids and Surfaces B: Biointerfaces. 2016, 141, 141-147. 
[33] Zhang Q, Iwakuma N, Sharma P, Moudgil B, Wu C, McNeill J, et al. Gold nanoparticles as a contrast agent for in vivo tumor imaging with photoacoustic tomography. Nanotechnology. 2009;20(39):395102.

[34] Labouta HI, Liu DC, Lin LL, Butler MK, Grice JE, Raphael AP, et al. Gold nanoparticle penetration and reduced metabolism in human skin by toluene. Pharmaceutical Research. 2011;28(11):2931-44.

[35] Labouta H, El-Khordagui L, Schneider M. Could chemical enhancement of gold nanoparticle penetration be extrapolated from established approaches for drug permeation? Skin Pharmacology and Physiology. 2012;25(4):208-18.

[36] Fernandes R, Smyth NR, Muskens OL, Nitti S, Heuer-Jungemann A, Ardern-Jones MR, et al. Interactions of skin with gold nanoparticles of different surface charge, shape, and functionality. Small. 2015;11(6):713-21.

[37] Larese Filon F, Crosera M, Adami G, Bovenzi M, Rossi F, Maina G. Human skin penetration of gold nanoparticles through intact and damaged skin. Nanotoxicology. 2011;5(4):493-501.

[38] Liu DC, Raphael AP, Sundh D, Grice JE, Soyer HP, Roberts MS, et al. The human stratum corneum prevents small gold nanoparticle penetration and their potential toxic metabolic consequences. Journal of Nanomaterials. 2012;2012:7.

[39] Geusens B, Van Gele M, Braat S, De Smedt SC, Stuart MC, Prow TW, et al. Flexible nanosomes (SECosomes) enable efficient siRNA delivery in cultured primary skin cells and in the viable epidermis of ex vivo human skin. Advanced Functional Materials. 2010;20(23):4077-90.

[40] Prow TW, Grice JE, Lin LL, Faye R, Butler M, Becker W, et al. Nanoparticles and microparticles for skin drug delivery. Advanced Drug Delivery Reviews. 2011;63(6): 470-91.

[41] Zhu Y, Choe C-S, Ahlberg S, Meinke MC, Alexiev U, Lademann J, et al. Penetration of silver nanoparticles into porcine skin ex vivo using fluorescence lifetime imaging microscopy, Raman microscopy, and surface-enhanced Raman scattering microscopy. Journal of Biomedical Optics. 2015;20(5):051006.

[42] Gittard SD, Miller PR, Boehm RD, Ovsianikov A, Chichkov BN, Heiser J, et al. Multiphoton microscopy of transdermal quantum dot delivery using two photon polymerization-fabricated polymer microneedles. Faraday Discussions. 2011;149:171-85.

[43] Larson DR, Zipfel WR, Williams RM, Clark SW, Bruchez MP, Wise FW, et al. Watersoluble quantum dots for multiphoton fluorescence imaging in vivo. Science. 2003;300(5624):1434-6.

[44] Lo W, Ghazaryan A, Tso C-H, Hu P-S, Chen W-L, Kuo T-R, et al. Oleic acid-enhanced transdermal delivery pathways of fluorescent nanoparticles. Applied Physics Letters. 2012;100(21):213701. 
[45] Boehm R, Miller P, Hayes S, Monteiro-Riviere N, Narayan R. Modification of microneedles using inkjet printing. AIP Advances. 2011;1(2):022139.

[46] Cheng S-H, Li F-C, Souris JS, Yang C-S, Tseng F-G, Lee H-S, et al. Visualizing dynamics of sub-hepatic distribution of nanoparticles using intravital multiphoton fluorescence microscopy. ACS Nano. 2012;6(5):4122-31.

[47] Semete B, Booysen L, Lemmer Y, Kalombo L, Katata L, Verschoor J, et al. In vivo evaluation of the biodistribution and safety of PLGA nanoparticles as drug delivery systems. Nanomedicine: Nanotechnology, Biology and Medicine. 2010;6(5):662-71.

[48] Johnston HJ, Semmler-Behnke M, Brown DM, Kreyling W, Tran L, Stone V. Evaluating the uptake and intracellular fate of polystyrene nanoparticles by primary and hepatocyte cell lines in vitro. Toxicology and Applied Pharmacology. 2010;242(1):66-78.

[49] Liang X, Grice JE, Zhu Y, Liu D, Sanchez WY, Li Z, et al. Intravital multiphoton imaging of the selective uptake of water-dispersible quantum dots into sinusoidal liver cells. Small. 2015;11(14):1711-20.

[50] Garrett NL, Lalatsa A, Uchegbu I, Schätzlein A, Moger J. Exploring uptake mechanisms of oral nanomedicines using multimodal nonlinear optical microscopy. Journal of Biophotonics. 2012;5(5-6):458-68.

[51] Chen A, Dogdas B, Mehta S, Haskell K, Ng B, Keough E, et al., editors. Quantification of $C y-5$ siRNA signal in the intra-vital multi-photon microscopy images. Engineering in Medicine and Biology Society (EMBC), 2012 Annual International Conference of the IEEE; 2012, San Diego, CA, USA.

[52] Loginova YF, Kazachkina NI, Zherdeva VV, Rusanov AL, Shirmanova MV, Zagaynova $\mathrm{EV}$, et al. Biodistribution of intact fluorescent $\mathrm{CdSe} / \mathrm{CdS} / \mathrm{ZnS}$ quantum dots coated by mercaptopropionic acid after intravenous injection into mice. Journal of Biophotonics. 2012;5(11-12):848-59.

[53] Liang Y, Shilagard T, Xiao SY, Snyder N, Lau D, Cicalese L, et al. Visualizing hepatitis $\mathrm{C}$ virus infections in human liver by two-photon microscopy. Gastroenterology. 2009;137(4):1448-58.

[54] Boreham A, Kim T-Y, Spahn V, Stein C, Mundhenk L, Gruber A, et al. Exploiting fluorescence lifetime plasticity in FLIM: target molecule localization in cells and tissues. ACS Medicinal Chemistry Letters. 2011;2(10):724-8. 
Chapter 6

\title{
Investigations on Dental Alloys Using Metallographic Observation, Scanning Electron Microscopy, and Energy- Dispersive X-Ray Spectroscopy
}

\author{
Lavinia Ardelean, Lucien Reclaru, \\ Cristina-Maria Bortun and Laura-Cristina Rusu
}

Additional information is available at the end of the chapter

http://dx.doi.org/10.5772/64291

\begin{abstract}
Alloys are used in various areas of dentistry, but mainly in prosthetics. Their properties, behavior, and corrosion resistance are of great importance for the success of the prosthetic treatment. Among the investigations used for assessing dental alloys, in this chapter, we focus on metallographic observation, scanning electron microscopy, and energy-dispersive $\mathrm{X}$-ray spectroscopy and present the ways we investigated several types of dental alloys. We obtained important data concerning their structure and corrosion potential that could explain certain shortcomings which appeared after luting of the fixed partial dentures.
\end{abstract}

Keywords: dental alloys, metallographic observation, scanning electron microscopy, energy-dispersive $\mathrm{X}$-ray spectroscopy, corrosion

\section{Introduction}

Alloys are used in various areas of dentistry, but mainly in prosthetics. Noble-metal alloys and base-metal alloys are used for manufacturing different prosthetic pieces: crowns, inlays, fixed partial dentures, and metallic frames of removable partial dentures. The field of dental alloys is a very extensive one, in constant development, encompassing both the materials themselves and the manufacturing methods [1].

In the past, the use of high-nobility alloys, with a high content of gold or noble metals, generally ensured quality for fixed partial dentures. However, later, the use of low-nobility alloys gained 
interest due to economic pressures. As a consequence of this trend, problems regarding chemical stability of this type of alloys have emerged. Accordingly, more attention was paid to the quantification of the chemical stability of dental alloys in terms of their corrosion behavior.

In time, the necessity to introduce a large number of alternative casting alloys of low cost has become more obvious. Base alloys containing nickel, chromium, cobalt, etc., and non-noble metals, also had to be accompanied by the continuous check of corrosion resistance to assure a long-term durability and good aesthetic result [2]. Classes of dental alloys used for dental applications are listed in Table 1.

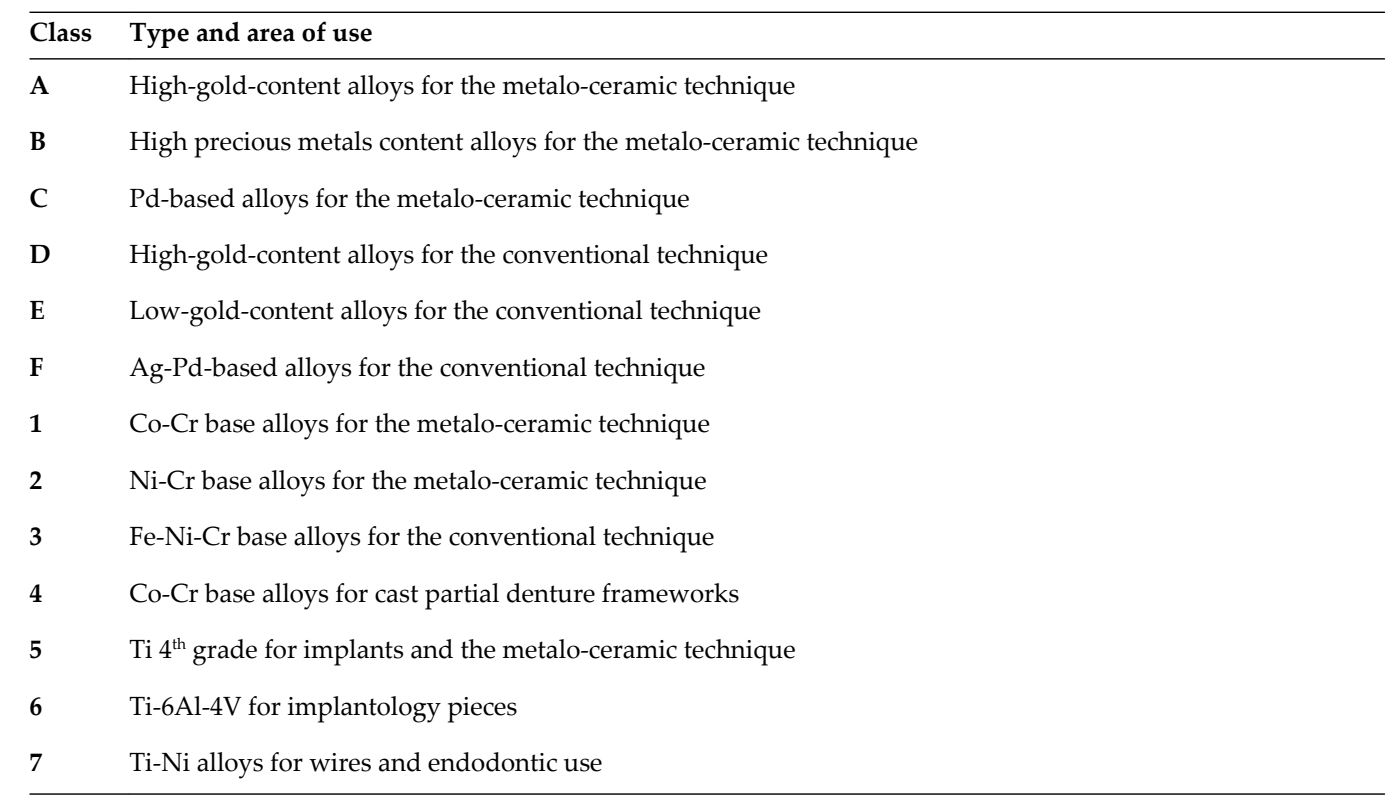

Table 1. Classes of dental alloys.

The alloy's microstructure is influenced by heat treatments. Varying the solidification rate and post-solidification anneals leads to the modification of the grain size. The homogenization anneals, alloy composition, and casting conditions influence the degree of segregation in a casting. Furthermore, the particularity of low-gold alloys is that they exhibit both precipitation and ordering reactions that affect their chemical stability. However, the desirable features of low-gold-content alloys are large grains, a minimum of segregation, and the absence of precipitates and ordered phases.

Cobalt-based alloys are often used to manufacture different types of devices implanted in the body. In dentistry [3, 4], cobalt-chromium alloys are mainly used for manufacturing removable partial dentures and metal ceramic fixed partial dentures, and also in fine framework constructions. These alloys are characterized by excellent corrosion resistance and superior mechanical properties [5]. Dental $\mathrm{Co}-\mathrm{Cr}$ alloys have diversified over time, aiming at creating 
both new products and new technologies to process them [6], including attempts to combine them with precious metals or to manufacture them by sintering instead of casting.

The alloy performance when processing is an important factor affecting its selection. Properties such as castability, surface roughness, and deformation resistance affect the usability of an alloy [7].

At present, there is no perfect dental alloy. Alloys are mixtures of two or more metals. Metals, when cooling after melting, usually result in a solid solution with higher values of hardness, resistance, and flexibility than the initial components. Due to their grain-type structure, alloys are predisposed to corrosion. The oral cavity provides an ideal environment for conducting electrochemical corrosion phenomena, which takes place in the presence of moisture. Other shortcomings involve the manufacturing technology, which, in case of casting, is quite laborious. Incorrect manufacturing technology or improper finishing may also contribute to corrosion debut.

Corrosion results in a visible alteration of the material and also affects the metallic device's function, so the major goal for any alloy used in the oral cavity is to have a good corrosion resistance.

The degree of corrosion damage on the prosthetic restoration depends on different factors such as the type of alloy, the presence of soft or hard deposits on its surface, the relation with the oral tissues, existing fractures of the veneering material, the age of the restoration, and the composition of saliva [7]. Alloy-related internal factors include composition, structure, impurities, and surface condition, and the external factors being electrolyte related (composition, concentration, temperature, oxygen content, $\mathrm{pH}$, heterogeneity, etc.) [8]. Parameters related to topography (surface ratio of the two materials, geometric distribution, etc.) may also play a role in developing corrosion.

Grain size and structural heterogeneity influence corrosion; impurities between grains and large grains are favourizing factors. The tendency to corrode is lower in case of a more homogeneous distribution of metallic atoms in the alloy. That is the reason why most alloys are submitted to a homogenizing heat treatment, to minimize the possibility of electrochemical corrosion.

The surface condition depends on the polishing degree, surface properties, and absence of pores or cracks in the primary protective layers [9].

The correlation between $\mathrm{pH}$ and corrosion may be summarized as follows: low in a neutral environment, generally high in an acidic environment, and variable in a basic environment. The increase of the electrode potential is frequently the consequence of an increased electrode potential [10]. The temperature's effect is an important but indirect one. Concentration of the metal's specific ions, in the vicinity of the electrode surface, is influenced by the movement of the solution and may cause variations. The process depends on the duration of exposure.

To assess the quality of a fixed partial denture and its corrosion potential, investigations such as metallographic observation, scanning electron microscopy (SEM), and energy-dispersive X-ray spectroscopy (EDX) are of much effectiveness. 
Metallography is essentially the study of the structural characteristics or constitution of a metal or an alloy in relation to its physical and mechanical properties. Besides the crystallographic nature of a material, imperfections inside a material have an even greater influence on the mechanical properties, that is, tensile, fatigue, creep, fracture toughness, and impact properties. Some defects such as missing atoms and dislocations cannot be observed optically except by their effects, that is, strain, etch pits, and slip lines.

Scanning electron microscopy, an electron microscopy technique based on the principle of electron-matter interactions, enabled us to produce high-resolution images of the sample's surface.

Energy-dispersive X-ray spectroscopy permitted us to do the elemental analysis or chemical characterization of the samples. Its characterization capabilities are due in large part to the fundamental principle that each element has a unique atomic structure, allowing unique set of peaks on its X-ray emission spectrum.

\section{Metallographic observation, scanning electron microscopy and energy- dispersive $X$-ray spectroscopy in corrosion behavior investigation of different types of dental alloys}

\subsection{Low-gold-content alloy}

In case of a fixed prosthetic restoration (Figure 1), made of a low-gold-content conventional class $\mathrm{E}$ alloy $(\mathrm{Au}, \mathrm{Ag}, \mathrm{Pd}, \mathrm{Cu}, \mathrm{Zn})$, a severe color change and the loss of the metallic shine occurred only a few months after luting, dark and shiny appearances or areas being present at the surface. Removing was decided in order to analyze the causes that lead to its failure.

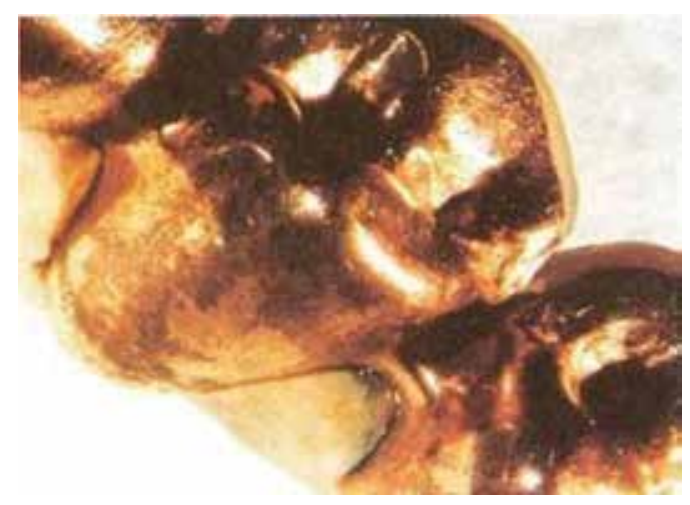

Figure 1. The deteriorated surface of the prosthetic restoration.

After removing it, we prepared two samples, one was used to analyze the damaged surface using SEM and EDX, without any surface alteration, and the other one was used to observe the superficial structure of the alloy, after transversally sectioning it. 


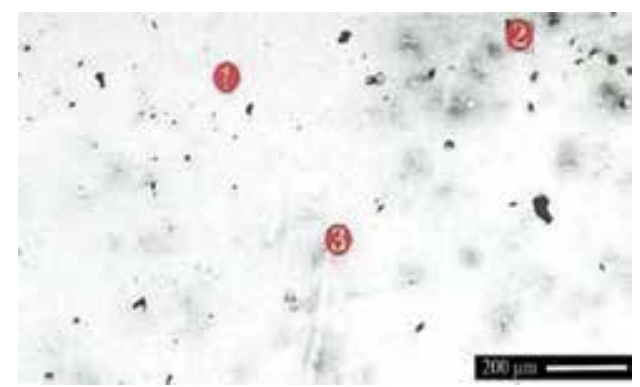

Figure 2. Surface image using retro diffused electrons (chemical contrast) of the sample.
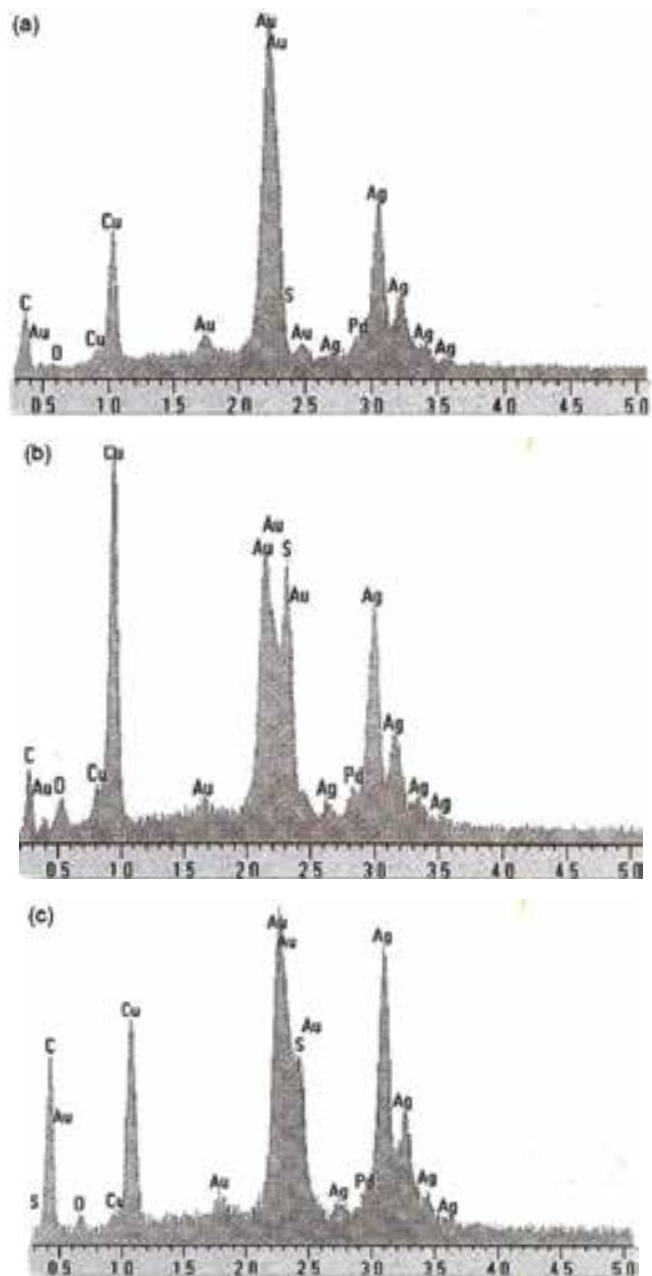

Figure 3. (a) EDX spectrum of area 1 (without sulfur). (b) EDX spectrum of area 2 (rich in copper and sulfur). (c) EDX spectrum of area 3 (rich in silver and sulfur). 


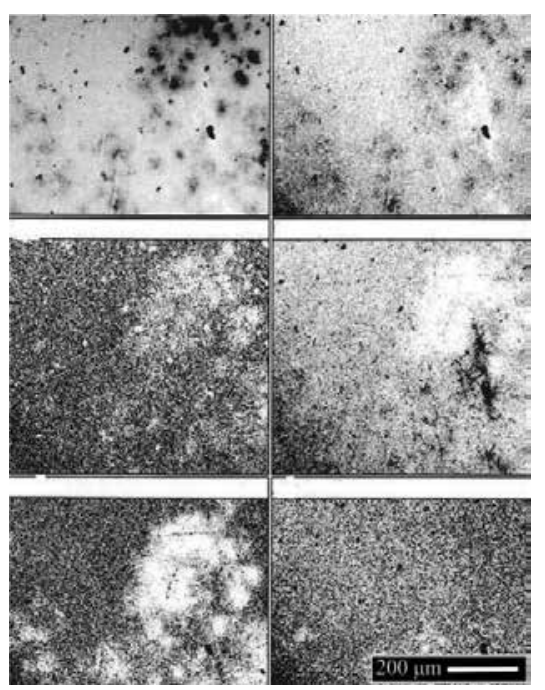

Figure 4. Retro diffused electron analysis (chemical contrast) of area 2 and allocation of the elements $(\mathrm{Au}, \mathrm{O}, \mathrm{Cu}, \mathrm{S}$, and $\mathrm{Ag})$.

The surface analysis with retro-diffused electrons (Figure 2) and EDX (Figure 3a-c) were performed. They indicate the correlation between coloration and a non-homogeneous surface composition. The presence of areas without sulfur (area 1, Figure 3a), areas rich in copper and sulfur (area 2, Figure 3b), and areas rich in silver and sulfur (area 3, Figure 3c) are shown.

Retro-diffused electron analysis and the location of the elements $(\mathrm{Au}, \mathrm{O}, \mathrm{Cu}, \mathrm{S}$, and $\mathrm{Ag}$ ) (Figure 4) show that the shine loss in area 2 is related to the presence of high amount of sulfur and copper. The presence of oxygen is related to copper localization.

SEM section analysis using secondary electrons (Figure 5a) reveals an important millimetric internal porosity, localized in the external part, in some areas merging into the surface. Figure $5 \mathrm{~b}$ reveals a dendritic non-homogeneous structure in the vicinity of the surface (SEM analysis with retro-diffused electrons).

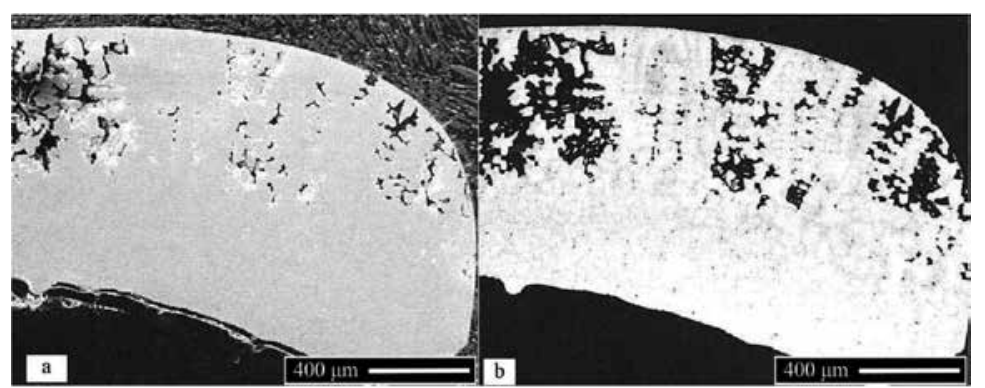

Figure 5. SEM analysis with (a) secondary electrons (topographic contrast) and (b) retro diffused electrons (chemical contrast) of the sample. 
$\mathrm{Au}, \mathrm{Ag}$, and $\mathrm{Cu}$ (main alloy elements) are positioned close to the surface and in the vicinity of the porous areas, in a non-homogeneous way. This non-homogeneity is more important in the sub-superficial darkened areas than in the sub-superficial shiny areas. The porosities are colored in black and are characterized by the presence of tiny inclusions very rich in $\mathrm{Cu}$ (Figure 6).

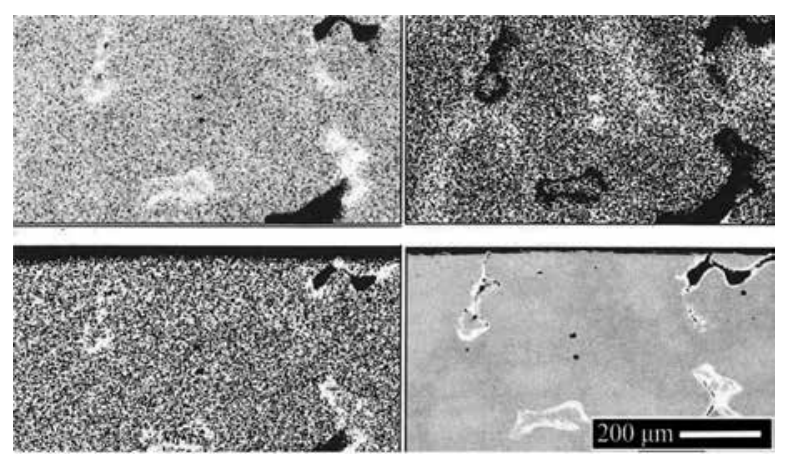

Figure 6. Observation of the sub-superficial layer of the dark area with retro diffused electrons (chemical contrast) and element allocation $(\mathrm{Au}, \mathrm{Ag}$, and $\mathrm{Cu})$.

The differences between the nominal chemical composition of the analyzed areas (areas I, II, III, and IV) are shown in Table 2. The four areas where the relative chemical composition in $\mathrm{Au}, \mathrm{Ag}$, and $\mathrm{Cu}$ was measured are as follows: I and II, internal composition of the matrix and external composition of the inclusions; III, inclusions rich in Au and Ag (Figure 4); and IV, inclusions rich in $\mathrm{Cu}$ (Figure 7). The corrosion resistance is negatively affected by any change in the alloy's composition (decreasing or increasing with one or more elements) [11], the corrosion being accelerated by the unstable phases in the alloy structure. In our case, the shine loss represents the result of selective corrosion subsequent to the appearance of a galvanic cell between these unstable phases and the alloy matrix [2]. Composition analysis reveals the presence of sulfur and oxygen. The presence of a high amount of copper or silver in the darkened areas is explained by their reaction with the sulfurous compounds in the saliva. Formation of insoluble chemical products (sulfides, copper, and silver) is involved in the selective corrosion process [12].

\begin{tabular}{llll}
\hline & $\mathbf{A u}^{*}$ & $\mathbf{A g}^{*}$ & $\mathbf{C u}^{*}$ \\
\hline I & 560 & 243 & 197 \\
II & 574 & 237 & 189 \\
III & 611 & 354 & 35 \\
IV & 253 & 123 & 624 \\
\hline
\end{tabular}

*\% weight

Table 2. Relative chemical compositions of the four areas. 

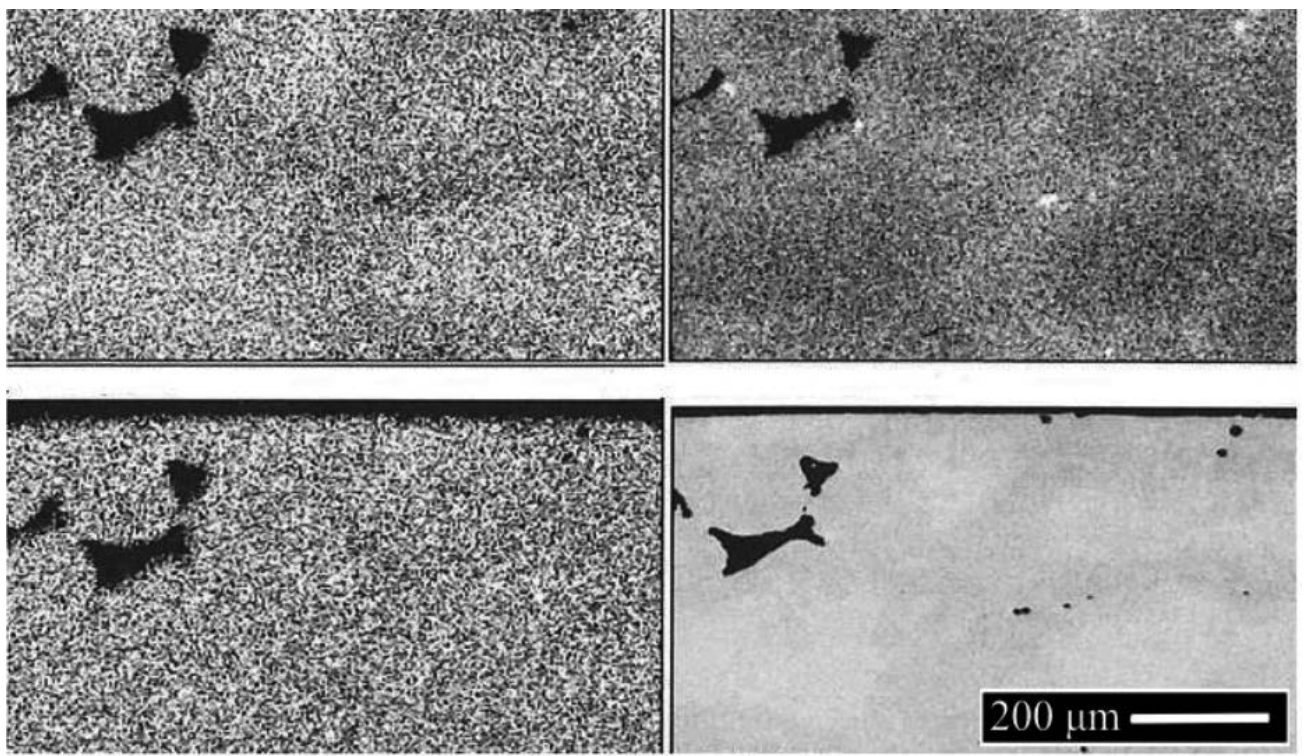

Figure 7. Observation of the sub-superficial layer of the shiny area with retro diffused electrons (chemical contrast) and the element allocation $(\mathrm{Au}, \mathrm{Ag}$, and $\mathrm{Cu})$.

The failure is subsequent to the incorrect alloy melting. This caused the internal porosities and areas with very different superficial compositions. Furthermore, the surface was not properly polished, causing surface porosity. The porous and chemically non-homogeneous surface, led to decreased corrosion resistance and subsequent degradation of the prosthetic restoration, in a short time after luting.

\subsection{High-gold-content alloy}

For determining corrosion causes of a fixed prosthetic restoration made of noble alloys with high content of gold, shortly after luting, we used SEM and EDX, applied to the restoration surface and in section. The fixed prosthetic restoration consisted of a cast part and a metalceramic part [13].

When superficially analyzing the cast part (Figure 8) using SEM, three different areas appeared.

- Areas with no deposits and free from corrosion deposits. EDX reveals a normal nominal alloy composition.

- Areas in bluish-black color, without deposits. In these areas, the surface analysis by laser ionization revealed the formation of copper and silver sulfides, concentration decreasing rapidly in the depth.

- Areas characterized by whitish deposits. In these areas, sulfur, sodium, and potassium chlorides are present, as well as calcium phosphates. A high concentration of copper was also noticed, compared to that measured at the nominal composition. 


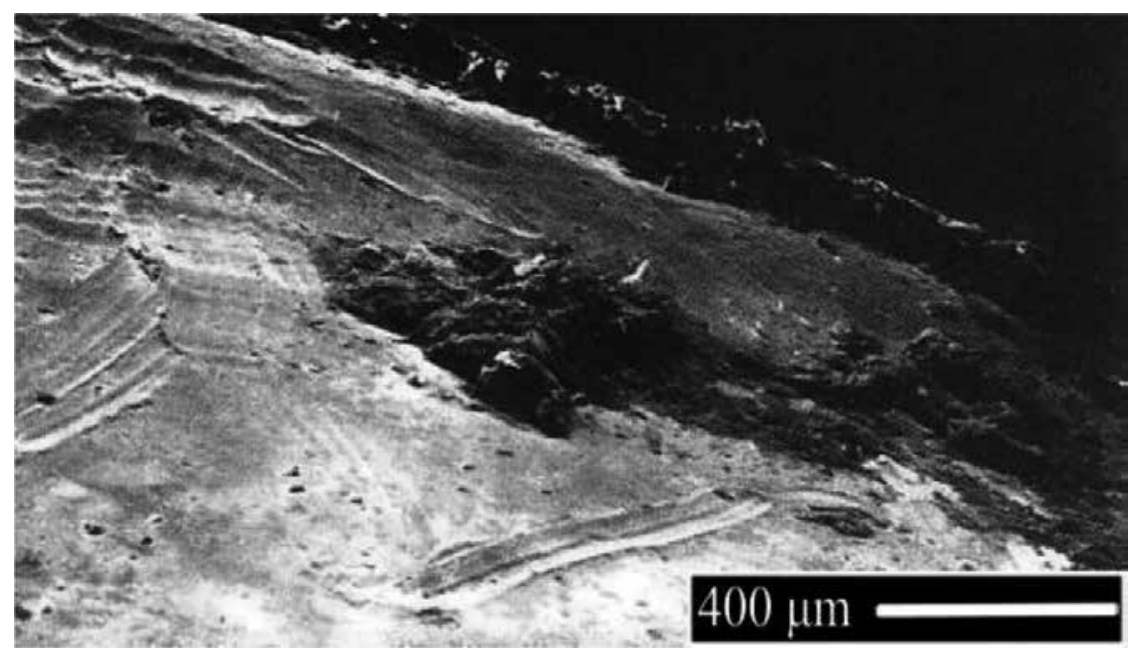

Figure 8. SEM observation (500× magnification) of the corroded crown surface.

Section analysis by EDX shows that the segregations are nodules of copper and zinc oxide. Metallographic observation of a section through the cast part reveals no defects in the internal structure of the alloy. On the other hand, SEM, with a 5000× magnification, shows an important segregation in the cast part (Figure 9).

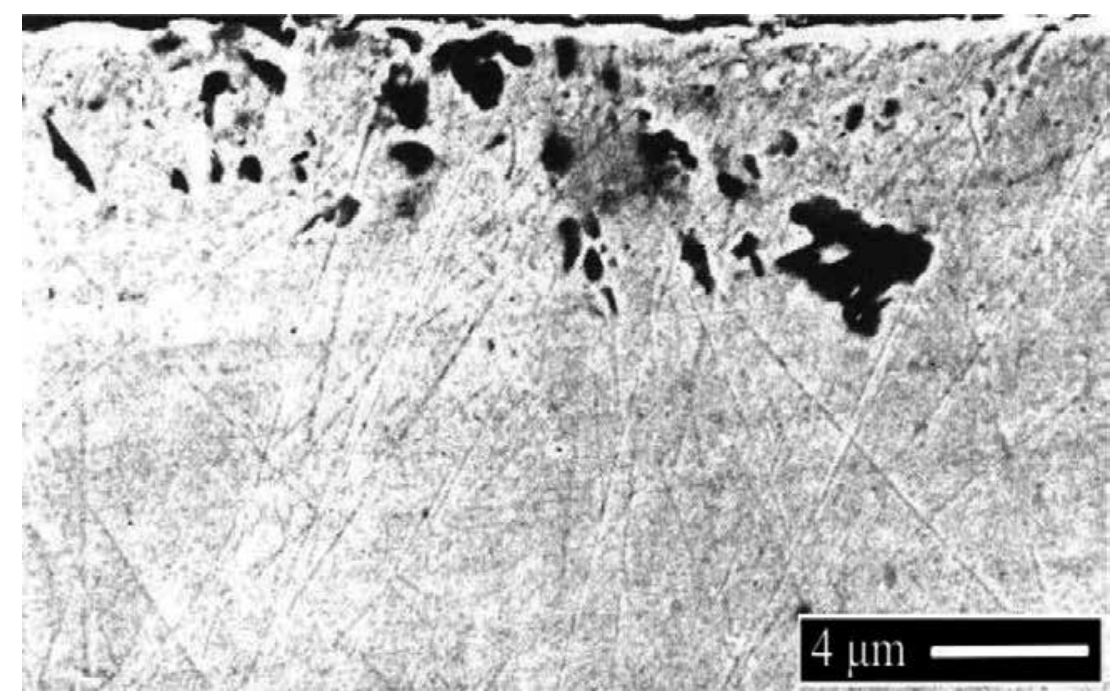

Figure 9. SEM observation (5000× magnification) of the cast part.

The nodules observed originate from the internal oxidation of the less noble alloy elements [12]. Our conclusion is that, in this case, we are dealing with localized corrosion in which the less noble parts of the surface consist of copper and zinc oxide nodules. 
Thus, a mixed potential (corrosion potential) has been created on the same surface due to the areas of different chemical composition [14], the anodic parts being areas consisting of copper and zinc oxide nodules. Corrosion was caused by the appearance of a mixed potential on the surface of the cast part as a consequence of incorrect manufacturing.

\subsection{Cobalt-chromium alloys doped with precious metals}

To improve corrosion resistance, a new generation of cobalt-chromium alloys doped with precious metals ( $\mathrm{Au}, \mathrm{Pt}$, and $\mathrm{Ru}$ ) was created [15]. We used EDX to determine the microstructure of this new generation of alloys by testing four different products in order to determine if they have improved corrosion resistance compared to classic $\mathrm{Co}-\mathrm{Cr}$ alloys. The alloys were also micrographically and electrochemically tested. The compositions of the four tested alloys and of a classic Co-Cr alloy are listed in Table 3.

\begin{tabular}{llllll}
\hline Element & Co-Cr & $\mathbf{1}$ & $\mathbf{2}$ & $\mathbf{3}$ & $\mathbf{4}$ \\
\hline $\mathrm{Co}$ & 63.7 & 63.5 & 52.0 & 50.6 & 59.3 \\
$\mathrm{Cr}$ & 28.9 & 21.0 & 25.0 & 18.5 & 25.0 \\
$\mathrm{Mo}$ & 5.3 & & 4.5 & 3.0 & 5.0 \\
$\mathrm{Ga}$ & & 4.5 & 6.0 & & 2.5 \\
$\mathrm{In}$ & & Trace & 5.0 & & 1.2 \\
$\mathrm{Au}$ & 2.0 & 2.0 & & 2.0 \\
$\mathrm{Pt}$ & & Trace & 2.0 & 15.0 & \\
$\mathrm{Ru}$ & & & 10.0 & \\
$\mathrm{Sn}$ & & 1.0 & & \\
$\mathrm{Mn}$ & & 0.5 & 1.0 & \\
$\mathrm{Si}$ & & & & \\
$\mathrm{W}$ & 0.8 & & & 0.75 & \\
$\mathrm{Nb}$ & & & & 0.5 & \\
$\mathrm{Al}$ & 0.1 & & & & \\
$\mathrm{Ti}$ & & & & & \\
$\mathrm{Fe}$ & & & & & \\
\hline
\end{tabular}

Table 3. Chemical composition of the tested alloys (wt \%).

The alloys were micrographically analyzed, analysis of phases by EDX was carried out, and hardness properties were also tested [15-17].

Metallographic structures of the tested alloys are shown in Figures 10-13. Phase compositions are listed in Tables 4-7. 


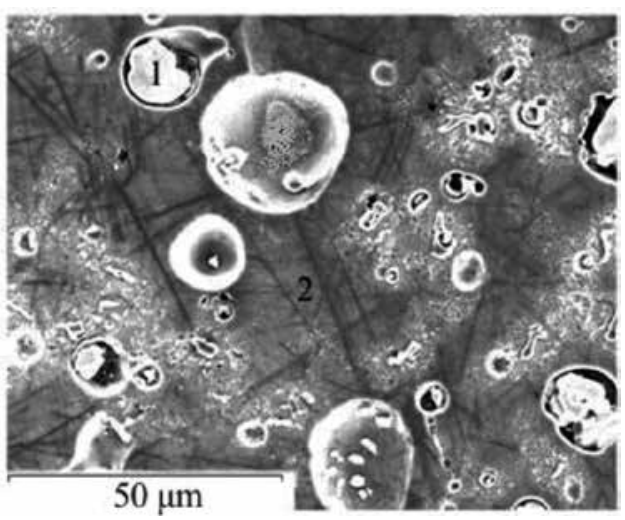

Figure 10. Microstructure of alloy 1.

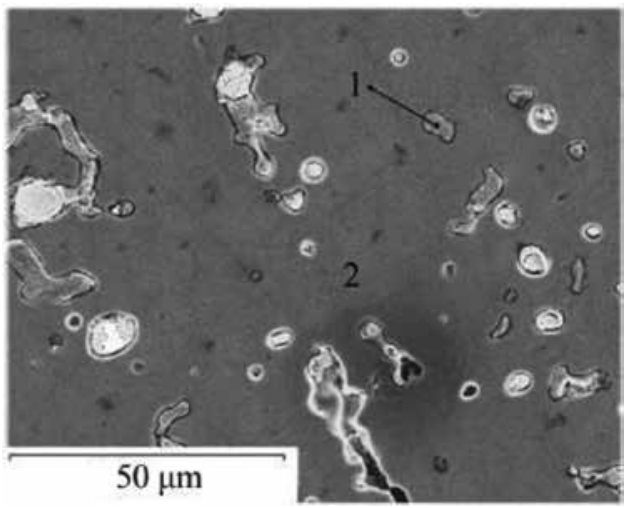

Figure 11. Microstructure of alloy 2.

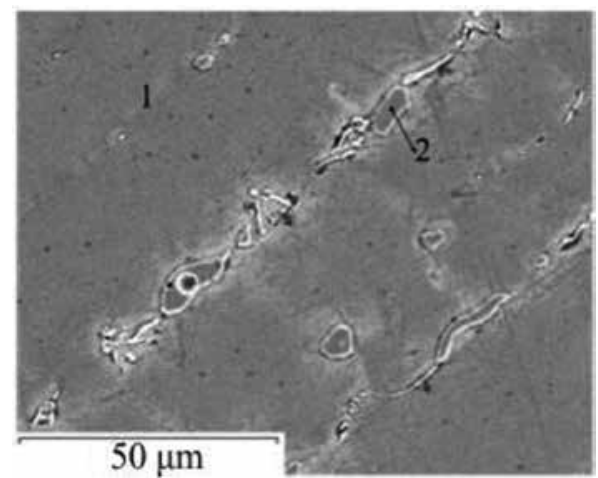

Figure 12. Microstructure of alloy 3. 


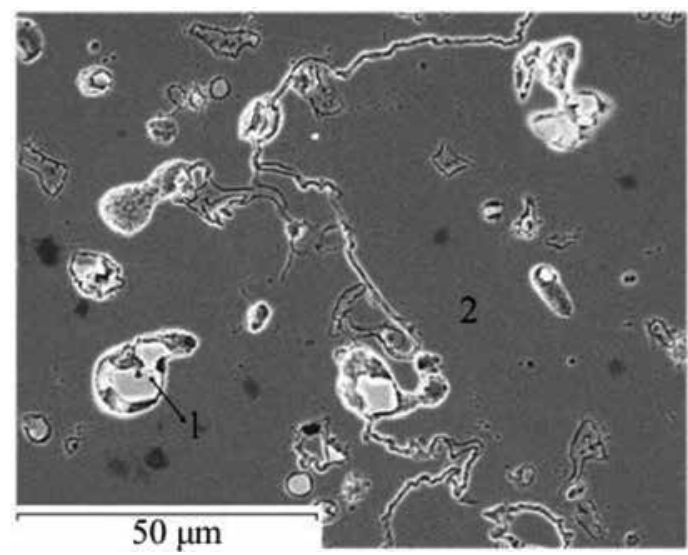

Figure 13. Microstructure of alloy 4.

\begin{tabular}{ccccccccccc}
\hline & Co & Cr & Mo & Si & Mn & Ga & In & Fe & Pt & Au \\
\hline $\mathbf{1}$ & 60.15 & 28.42 & 4.39 & 1.06 & 0.50 & 4.89 & - & 0.58 & - & - \\
$\mathbf{2}$ & 1.30 & 0.62 & - & - & - & - & 42.77 & - & 28.31 & 27.01 \\
\hline
\end{tabular}

Table 4. Phase composition (wt \%) of alloy 1.

\begin{tabular}{ccccccccccc}
\hline & Co & Cr & Mo & Si & Ti & W & Ga & In & Pt & Au \\
\hline $\mathbf{1}$ & 63.48 & 25.71 & 3.55 & - & - & 4.85 & 2.41 & - & - & - \\
$\mathbf{2}$ & 28.46 & 32.83 & 22.98 & 1.74 & 0.95 & 11.31 & - & - & 1.73 & - \\
\hline
\end{tabular}

Table 5. Phase composition (wt \%) of alloy 2.

\begin{tabular}{ccccccccccc}
\hline & Co & Cr & Mo & Si & Mn & W & Ti & Nb & Pt & Ru \\
\hline $\mathbf{1}$ & 48.96 & 17.48 & 3.44 & 1.02 & 0.70 & 1.25 & - & - & 16.44 & 10.72 \\
$\mathbf{2}$ & 42.75 & 19.51 & 11.76 & 4.36 & 0.88 & 1.56 & - & - & 8.25 & 10.93 \\
\hline
\end{tabular}

Table 6. Phase composition (wt \%) of alloy 3.

\begin{tabular}{ccccccccc}
\hline & Co & Cr & Mo & Si & Ga & In & Pt & Au \\
\hline $\mathbf{1}$ & 59.61 & 28.04 & 5.37 & 0.63 & 6.35 & - & - & - \\
$\mathbf{2}$ & 1.44 & 0.76 & - & - & - & 51.17 & 28.87 & 17.77 \\
\hline
\end{tabular}

Table 7. Phase composition (wt \%) of alloy 4. 
The microstructures of alloys 1 and 4 are characterized by round inclusions. The diameter of these inclusions is up to $0.1 \mathrm{~mm}$. The chemical analysis of these zones showed In (between 42 and 51\%), Pt (around 28\%), and $\mathrm{Au}$ (between 18 and 27\%). The Vickers tests of the zones with inclusions for alloy 4 showed a mean hardness value of $147 \mathrm{HV}$. Compared to the overall hardness value of the alloy (326 HV) this is more than twice lower [15-17]. The hardness values are given in Table 8.

\begin{tabular}{llllll}
\hline Alloys & 1 overall & 2 overall & 3 overall & 4 overall & 4 zone 2 \\
\hline $\mathrm{HV}_{0.2}$ & 333 & 435 & 338 & 326 & 147 \\
\hline
\end{tabular}

Table 8. Vickers hardness of the tested alloys $(n=5)$.

For electrochemical measurements, artificial saliva of the Fusayama type was used, with the rotating electrode technique. The cathodic and anodic potentiodynamic polarization curves were measured from $1000 \mathrm{mV}$ to $+1250 \mathrm{mV}$ vs. saturated calomel electrode and showed important differences in the behavior of all the four studied alloys, compared to the classic Co$\mathrm{Cr}$ alloy. The worst behavior was shown by the alloys 1 and 4 that contain only gold. This confirms the existing results which state that $\mathrm{Au}$ is not miscible to $\mathrm{Co}$ and $\mathrm{Cr}$ [18].

Alloys 1 and 4 are characterized by a very complex microstructure. The round inclusions with a diameter up to $0.1 \mathrm{~mm}$ are in part non-miscible phases with a very low corrosion resistance. The classic Co-Cr alloy has the best corrosion behavior, followed by alloys 2 and 3 (addition of, respectively, $4 \%$ and $25 \%$ precious metals). Alloys 1 and 4 (with only addition of $2 \% \mathrm{Au}$ ) have the worst behavior. Adding of precious metals deteriorates the corrosion behavior of Co$\mathrm{Cr}$ alloys in a significant way. Gold doping, in particular, produces heterogeneous microstructures that are vulnerable to corrosive attack $[15,16,19]$.

In conclusion, $\mathrm{Co}-\mathrm{Cr}$ dental alloys doped with precious metals do not make sense, due to their poor behavior; using them as an alternative to conventional $\mathrm{Co}-\mathrm{Cr}$ alloys is not justified [15$17,19]$.

\subsection{Laser-sintered cobalt-chromium prosthetic elements}

Co-Cr alloys are traditionally manufactured by casting. Alternative modern technologies include the CAD/CAM systems. In this case, a ring-shaped material is used and most of the alloy is therefore lost, only a limited part of the material being actually used [20,21]. Another alternative modern technique that does not have the disadvantage of losing material is the laser sintering.

By means of a laser, using a well-established energy/local surface unit, a fine alloy powder is locally sintered to form the prosthetic element (Figures 14 and 15) [22, 23]. 


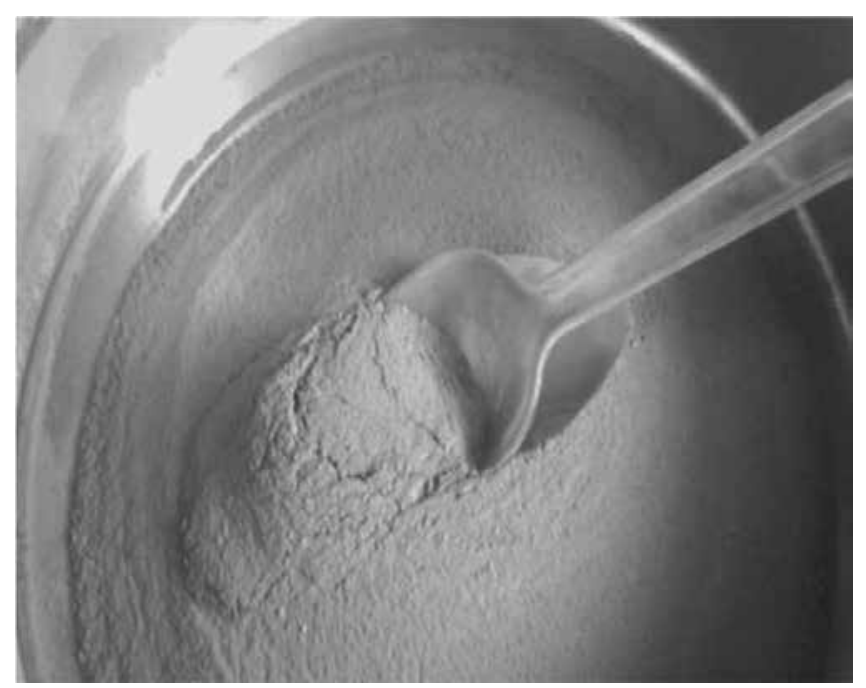

Figure 14. Co-Cr powder for sintering.

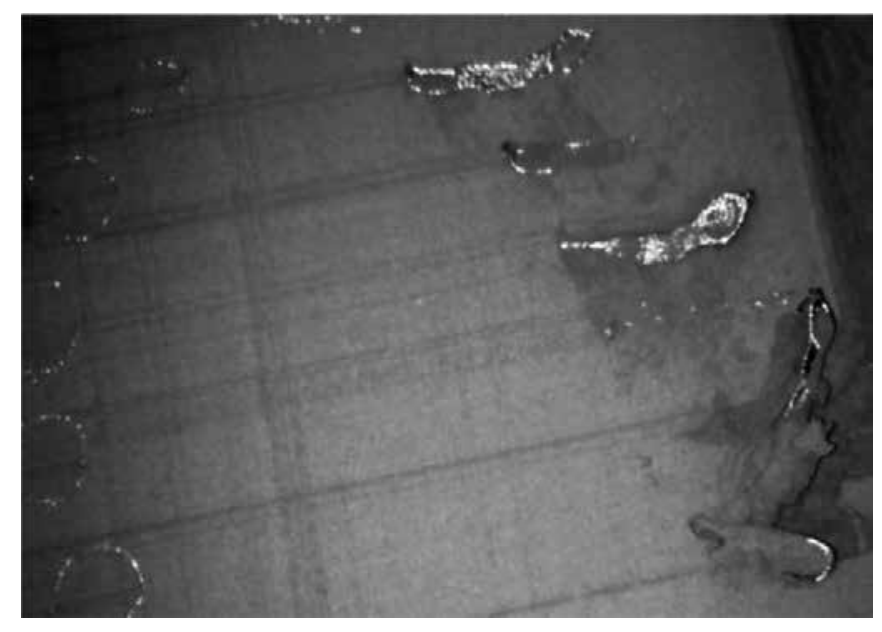

Figure 15. Selectively laser fusing the metal powder.

The topography of the respective restorations is designed by numerical monitoring after having scanned the devised objects. Several objects are numerically programmed on the tray. Subsequently, a computer-based program orders the laser beam. The laser is programmed in a way that it only becomes active at the site where the elements should be achieved. The alloy particles will consequently be sintered by the laser energy (Figure 16) [22, 23].

The programmed objects, virtually presented, are obtained by superposing several layers, a process during which, after each period of laser exposure, the tray is raised by $60-80 \mu \mathrm{m}$ and is subjected to another laser exposure after the application of each additional layer of powder. 
Thus, the whole reconstruction process is achieved layer by layer. This results in a fast prototype making process (Figure 17) [22, 23].

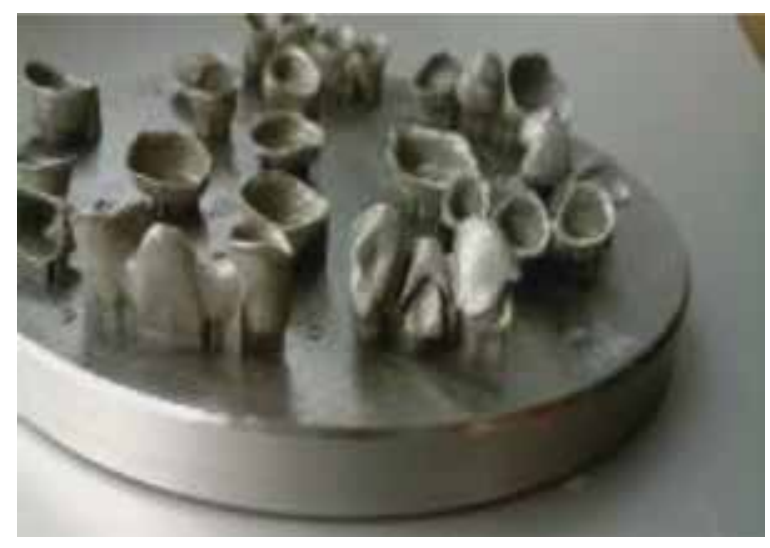

Figure 16. The bridge, immediately after separation from the tray, on the model.

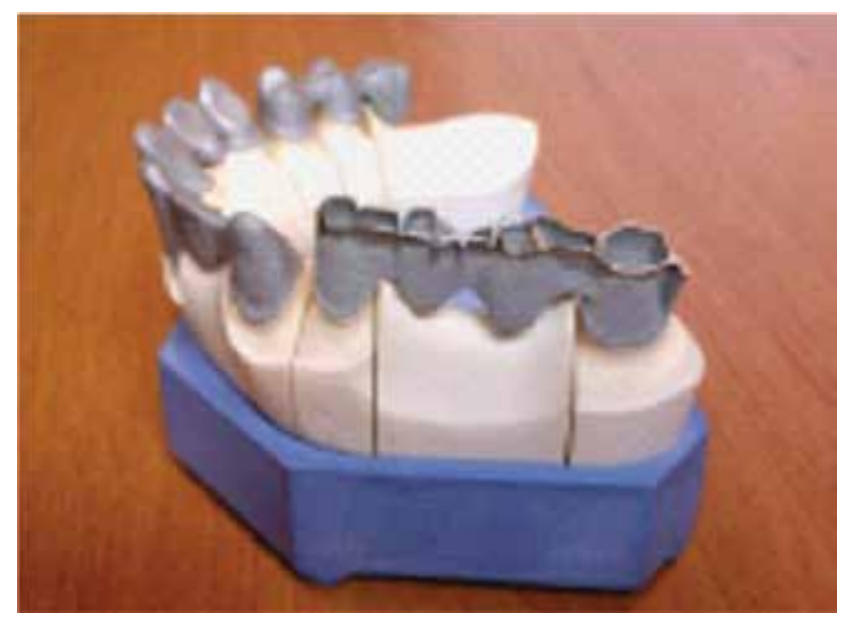

Figure 17. Co-Cr sintered frameworks.

The powder has to be as spherical as possible and its granulometry has to be below 20-30 $\mu \mathrm{m}$, which represents the space between the sintered layers. The composition of the alloy used by us is $64-67 \% \mathrm{Co}, 28-30 \% \mathrm{Cr}$, and $5-6 \% \mathrm{Mo}$.

After sintering, the prosthetic elements are cut down from the tray. The result is clinical satisfactory, with a precision of $25 \mu \mathrm{m}$ (Figure 16). The surface has a stratified structure matching the granulometry of the basic powder (Figure 18). From the mechanic point of view, the breaking limits of the sintered metalo-ceramic elements are comparable to casted metaloceramic elements, the average hardness being $395 \mathrm{HV}$. 


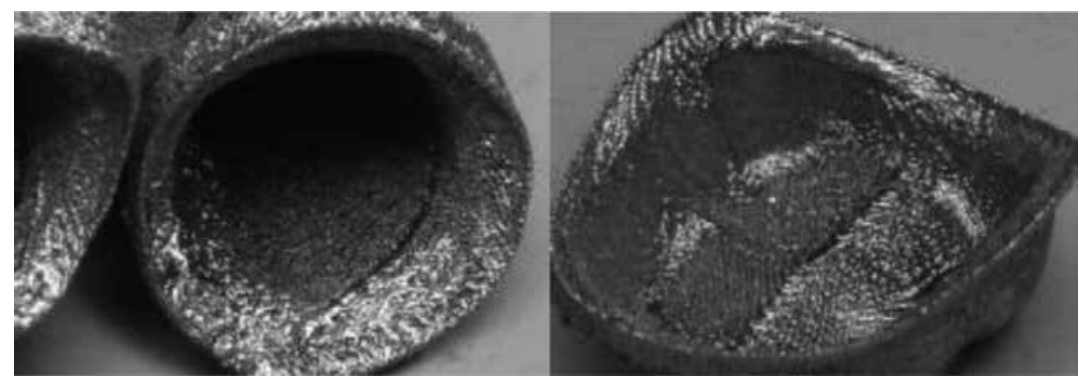

Figure 18. The internal surface of two crowns, immediately after sintering.

The metallographic observation (50× magnification) shows a slight porosity in the horizontal sample (compared to the sintering plane) (Figure 19), while in vertical sample, the pore lines are uninterrupted (Figure 20).

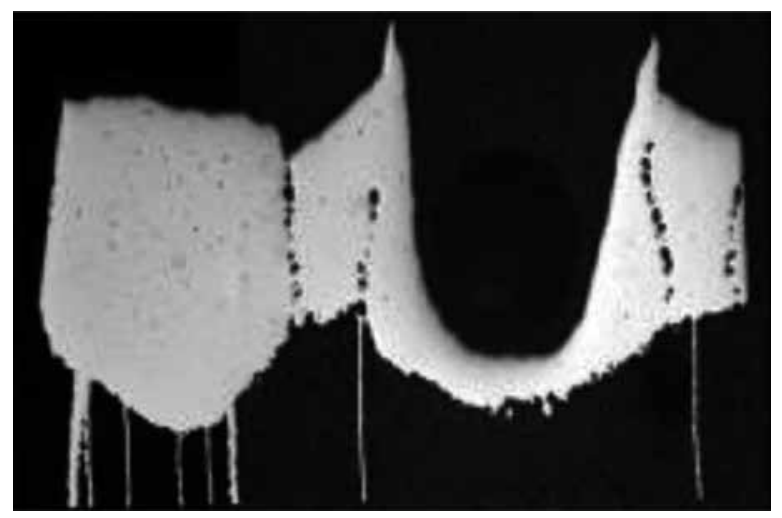

Figure 19. Metallographic observation of a horizontal sample, without chemical attack.

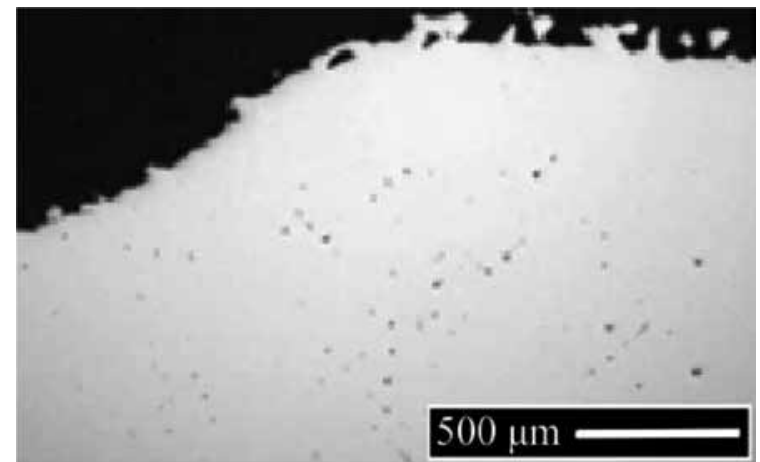

Figure 20. Metallographic observation of a vertical sample. 
The chemical attack shows (50× magnification), similar to the surface, a very fine layered structure roughly corresponding to the original granular structure of the base powder (Figure 21).

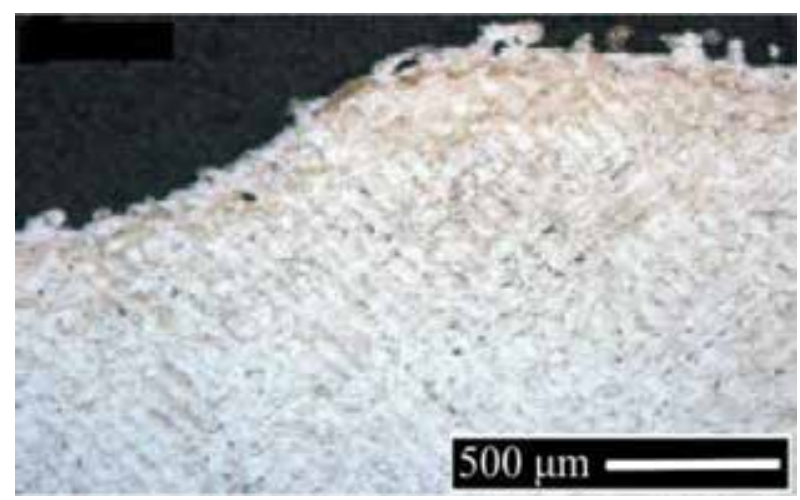

Figure 21. Metallographic observation of a horizontal sample, after chemical attack.

The local chemical composition is characterized by a high regularity: Co between 62.6 and $64.1 \%$, Cr between 29.3 and 30.5\%, and Mo between 4.9 and $6.4 \%$. Mn and Si are less than $1 \%$.

Our conclusion was that the laser sintering technique makes possible the manufacture of extremely accurate prosthetic elements with mechanical properties that correspond to any clinical requirement, but it seems to be a rather significant risk of internal porosity that might lead to fracture, cracking, or corrosion [22, 23].

\section{Conclusions}

The biocompatibility of dental casting alloys is of great importance because these alloys are in long-term intimate contact with oral tissues [24].

Alloys used for fixed prosthetic restorations must have an appropriate corrosion resistance to avoid the release of cytotoxic or sensitizing elements into the biological milieu [25]. The manufacturing conditions are of great importance as well as the environmental ones. The corrosion of dental alloys may be significantly increased by improper processing (formation of pits and crevices). Good corrosion resistance is an important criterion for alloy selection. Corrosion influences other properties of an alloy, such as esthetics and strength.

Electrochemical corrosion of alloys involves the ionization of elements that are released into the environment, for example, saliva [26], initially uncharged elements loose electrons and become positively charged ions as they are released into solution. The released elements may or may not cause problems [27]. One of the main factors that influence element release is the alloy's composition. Elemental release and corrosion occurs regardless of type or composition of the alloy. The elements with higher tendencies to be released include copper, zinc, and 
nickel. Gold and palladium show a low release tendency [27]. Element lability is also influenced by the phase structure of the alloy. If multiple phases are present, the risk of element release is greater because of the potential electrochemical corrosion among the phases [28]. The element release is also influenced by the surface characteristics of the alloy as roughness and the presence of oxides. Surface roughness increases elemental release because it exposes more atoms to the external environment. The oral environment also plays a role in corrosion. Low $\mathrm{pH}$ increases corrosion, especially when nickel-based alloys are involved. Corrosion is also particularly high in retentive areas such as crevices, pits, gingival, and sulcus [29].

The surface composition of the alloy may vary from the composition of the bulk of the alloy [30]. The surface composition may have a direct influence on which elements are released [31, 32]. Base dental alloys ( $\mathrm{Co}-\mathrm{Cr}$ ) have an inferior corrosion resistance than the noble alloys.

To determine the degree of internal porosity, in case of a major surface porosity we used metallographic observation. Failure due to incorrect alloy usage may occur when the alloy's normal melting conditions are not respected. This incorrect melting may be the cause for the internal porosities and for the areas with very different superficial compositions. A not properly polished surface, porous and chemically non-homogeneous, may be a result of incorrect melting and surface treatment, and leads to decreased corrosion resistance and subsequent degradation, after luting.

We also used it to determine the quality of laser-sintered $\mathrm{Co}-\mathrm{Cr}$ prosthetic elements, in horizontal and vertical samples, with and without chemical attack. Our conclusion was that there seems to be a rather significant risk of internal porosity which might lead to fracture, cracking, or corrosion.

For determining the corrosion causes of a fixed prosthetic restoration made of noble alloys, with high content or low content of gold, shortly after luting, we used scanning electron microscopy and energy-dispersive $\mathrm{X}$-ray spectroscopy, applied to the restoration surface and in section. Using SEM we were capable of producing high-resolution images of the surface of a sample. SEM limits itself to solid samples of certain size, but this was not an issue in our case. In case of EDX, the accuracy of the spectrum can be affected by various factors, including the nature of the sample, but generally has a statistical precision of $\pm 1 \%$.

EDX enabled us to do the elemental analysis or chemical characterization of the samples and was also used to determine the microstructure of the new generation of cobalt-chromium alloys doped with precious metals $(\mathrm{Au}, \mathrm{Pt}, \mathrm{Ru})$ by testing four different such alloys to determine if they have improved corrosion resistance compared to classic $\mathrm{Co}-\mathrm{Cr}$ alloys. We concluded that attempts to obtain alloys with better properties by doping $\mathrm{Co}-\mathrm{Cr}$ alloys with precious metals were not successful. Some cheaper alloys such as copper, aluminum, and bronze, also developed to substitute gold dental alloys, proved to be a great failure.

For minimal biological risks, dentists should consider alloys with low corrosion potential, mainly high-noble metal or noble alloys with single-phase microstructures. The alloy should be selected for each patient using corrosion and biologic data from the manufacturer [27]. Success or failure of a prosthetic restoration depends on many factors: the producer, the proper manufacture, and some related to the patient itself. 


\section{Author details}

Lavinia Ardelean ${ }^{1 *}$, Lucien Reclaru ${ }^{2 *}$, Cristina-Maria Bortun ${ }^{3}$ and Laura-Cristina Rusu ${ }^{1}$

*Address all correspondence to: lavinia_ardelean@umft.ro

1 Department of Technology of Dental Materials and Devices in Dental Medicine, “Victor Babes" University of Medicine and Pharmacy, Timisoara, Romania

2 Consultant Corrosion \& Biocompatibility Department, VVSA, Branch of Richemont International SA Varinor Innovation, Switzerland

3 Department of Dentures Technology, "Victor Babes" University of Medicine and Pharmacy, Timisoara, Romania

\section{References}

[1] Ardelean L, Rusu LC. Materials, instruments and devices in the dental laboratory. Timisoara: Eurostampa; 2013

[2] Susz C, Reclaru L, Ardelean L, Ghiban B, Rusu L. Dental Alloys. Timisoara: Victor Babes; 2010

[3] Ghiban B, Bortun C. Cobalt dental alloys. Bucuresti: Printech; 2009.

[4] Reclaru L, Eschler PY, Reto L, Blatter A. Electrochemical corrosion and metal ion release from Co-Cr-Mo prosthesis with titanium plasma spray coating. Biomaterials 2005;26(23): 4747-4756.

[5] Mareci D, Nemtoi G, Aelenei N, Bocanu C. The electrochemical behaviour of various non-precious $\mathrm{Ni}$ and $\mathrm{Co}$ based alloys in artificial saliva. European Cells \& Materials 2005;8(10): $1-7$.

[6] Gupta KP. The Co-Cr-Mo system. Journal of Phase Equilibria and Diffusion 2005;26(1): 87-92.

[7] Laurent F, Grosgogeat B, Reclaru L, Dalard F, Lissac M. Comparison of corrosion behavior in presence of oral bacteria. Biomaterials 2001;22(16): 2273-2282.

[8] Galo R, Ribeiro RF, Rodrigues RC, Rocha LA, de Mattos MGC. Effects of chemical composition on the corrosion of dental alloys. Brazilian Dental Journal 2012;23(2): 141148.

[9] Reclaru L, Ardelean L, Rusu LC. Surface condition influence on galvanic corrosion. Medicine in Evolution 2013;19(1): 152-157. 
[10] Bayramoglu G, Alemdaroglu T, Kedici S, Aksüt AA. The effect of pH on the corrosion of dental metal alloys. Journal of Oral Rehabilitation 2000;27(7): 563-575.

[11] Ardelean L, Reclaru L, Rusu LC. Metallographic observation to determine a lowcontent gold alloy castability. Revista de Chimie 2015;66(4): 584-586.

[12] Ardelean L, Reclaru L, Susz C, Rusu LC. Scanning electron microscopy and energydispersive X-ray spectroscopy in determining the metallic-shine-loss causes of a low gold content conventional alloy. Revista de Chimie 2012;63(2): 232-234.

[13] Reclaru L, Ardelean L, Rusu LC. Scanning electron microscopy and energy-dispersive X-ray spectroscopy in determining corrosion causes of a fixed prosthetic restoration. Revista de Chimie 2013;64(5): 545-547.

[14] Pop DM, Rominu M, Topala FI, Sinescu C, Dodenciu D, Rominu RO, Ardelean L, Rusu LC, Andoni M, Petrescu EL, Negrutiu ML. Laser weldings versus electrical weldings in dental technology. A corrosion approach study, Revista de Chimie 2011;62(12): 12031205.

[15] Ardelean L, Reclaru L, Bortun CM, Rusu LC. Assessment of Dental Alloys by Different Methods. In: Aliofhazraei M. (ed.) Superalloys. Rjeka: Intech; 2015. 141-170.

[16] Reclaru L, Luthy H, Eschler PY, Blatter A, Susz C. Corrosion behaviour of cobaltchromium dental alloys doped with precious metals. Biomaterials 2005;26(21): 43584365 .

[17] Ardelean L, Reclaru L, Bortun C, Ghiban B, Rusu L. New aspects concerning Co-Cr alloys. Metalurgia International 2010;15(9): 31-35.

[18] Kappert HF, Schuster M. The rule of precious alloys. Dental Labor 2000;43(3): 352-354.

[19] Reclaru L, Ardelean L, Rusu LC. Cobalt-chromium dental alloys doped with gold or platinum. Medicine in Evolution 2010;16(1): 69-72.

[20] Rusu LC, Ardelean L. CAD/CAM technology concerning biocompatibility in zirconia all-ceramic restorations, Revista de Chimie 2012;63(5): 513-515.

[21] Ardelean L, Rusu LC. Technological aspects of zirconia CAD/CAM all-ceramic restorations. Medicine in Evolution 2012;18(1): 149-154.

[22] Reclaru L, Ardelean L, Rusu L, Sinescu C. Co-Cr material selection in prosthetic restoration: laser sintering technology. Solid State Phenomena 2012;188:412-415.

[23] Reclaru L, Ardelean L, Rusu LC. Cobalt-Chromium dental alloys doped with gold or platinum. Medicine in Evolution 2010;16(1):69-72.

[24] Reclaru L, Ardelean L, Govor I, Tigmeanu CV, Rusu LC. In vitro toxicity testing of palladium using trifluorothymidine. Revista de Chimie 2014;65(10): 1149-1153. 
[25] Eschler PY, Lüthy H, Reclaru L, Blatter A, Loeffel O, Süsz C, Boesch J. Copperaluminium bronze-a substitute material for Gold dental alloys? European Cells and Materials 2003;5(1): 49-50.

[26] Craig RG, Powers JM. Restorative Dental Materials, 11th edn. St. Louis: Harcourt Health Sciences; 2002.

[27] Schmalz G, Arenholt-Bindslev D. Biocompatibility of Dental Materials. SpringerVerlag: Berlin/Heidelberg; 2009.

[28] Wataha JC. Principles of biocompatibility for dental practitioners. Journal of Prosthetic Dentistry 2001;86(2): 203-209.

[29] Reclaru L, Unger RE, Kirkpatrick CJ, Susz C, Eschler PY, Zuercher MH, Antoniac I, Luthy H. Ni-Cr based dental alloys; Ni release, corrosion and biological evaluation. Materials Science and Engineering 2012;32(6): 1452-1460.

[30] Messer RLW, Lucas C. Cytotoxicity of nickel-chromium alloys: bulk alloys compared to multiple ion salt solutions. Dental Materials 2000;16(3): 207-212.

[31] Chen CC, Chen RGS, Huang ST. Effect of chemical composition on the corrosion behavior of Ni-Cr-Mo dental casting alloys. Journal of Biomedical Materials Research. 2002;60(3): 458-465.

[32] Benatti OFM, Miranda WG, Muench A. In vitro and in vivo corrosion evaluation of nickel-chromium- and copper-aluminum-based alloys. Journal of Prosthetic Dentistry. 2000;84(3): 360-363. 


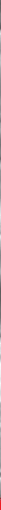

\section{Edited by Stefan G. Stanciu}

Countless healthcare and biomedical solutions with high impact in terms of timely diagnostics, therapeutic success, patient comfort or financial sustainability of healthcare systems rely on micro- and nanotechnologies. Thus, it is not at all exaggerate to claim that such technologies play in current days a tremendous role with respect to improving the quality of our life, health and well-being, which are the main priorities of modern science. This volume illustrates these statements, addressing highly significant scientific subjects from diverse areas of micro- and nanotechnologies for biotechnology. Authoritative voices in their fields present in this volume their work, or review recent trends, concepts and applications, in a manner that is accessible to a broad readership audience from both within and outside their specialist area.

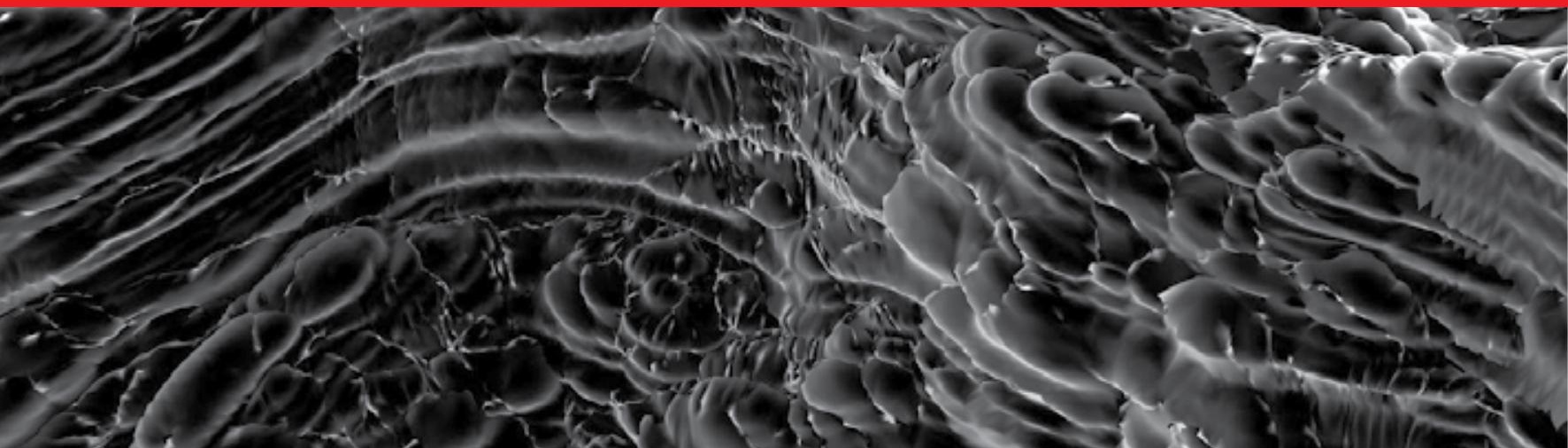

